\title{
The Evolution of Hurricane Humberto (2001)
}

\author{
A DISSERTATION SUBMITTED TO THE GRADUATE DIVISION OF \\ THE UNIVERSITY OF HAWAI'I AT MĀNOA IN PARTIAL \\ FULFILLMENT OF THE REQUIREMENTS FOR THE DEGREE OF
}

DOCTOR OF PHILOSOPHY

IN

METEOROLOGY

DECEMBER 2010

\author{
By \\ Klaus P. Dolling \\ Dissertation Committee: \\ Gary M. Barnes, Chairperson \\ Steven Businger \\ Pao-Shin Chu \\ Thomas A. Schroeder \\ Janet Becker
}




\section{ACKNOWLEDGMENTS}

I would like to thank Dr. Gary Barnes for his guidance and for improving my dissertation through our numerous consultations, constructive criticism, and his meticulous revisions. I would also like to thank Dr. Steven Businger, Dr. Pao-Shin Chu, Dr. Thomas Schroeder, and Dr. Janet Becker for their helpful suggestions and for serving on my dissertation committee. A special thanks to the Hurricane Research Division and NASA for conducting a methodical experiment with copious amounts of data, without which a detailed analysis of Humberto would have been impossible.

Heartfelt thanks to my family; Catherine Kerelejza, Maryann, Ulf, Charlotte, Stephan, Maxwell, and Ella Dolling for all their support throughout this process. Your encouragement has been an essential part of my success. I would also like to thank my classmates for valuable discussions and for their friendship. All of you have made my time here at the University of Hawaii a fulfilling and rich experience. 


\begin{abstract}
The causes of intensity change in tropical cyclones have continued to be a major challenge for the meteorological community. A lack of sampling throughout the life cycle of a tropical cyclone has contributed to our lack of understanding. In September of 2001, NOAA and NASA marshaled their resources and deployed over 200 Global Positioning System dropwindsondes within a $300 \mathrm{~km}$ radius of Tropical Cyclone Humberto on 3 consecutive days in the Convection and Moisture Experiment. This dissertation presents the results of an observational study of the aforementioned storm, combining aircraft data with the Global Positioning System dropwindsondes, airborne expendable bathythermographs, lower fuselage, and tail radar.
\end{abstract}

Kinematic and thermodynamic structures are examined in an attempt to better understand intensification processes for a high latitude storm traveling over a variable SST field, in a dry environment, and under increasing vertical wind shear. As Humberto is sampled as a tropical storm, the warm core develops from an area of subsidence on the trailing edge of an anvil. This warming acts to cap the boundary layer and allows for an increase in the energy content under the nascent eye. Throughout the experiment, thermodynamic and kinematic variables exhibit persistent relationships with the vertical wind shear vector. Vertical cross-sections of the warm core show that it has the highest temperature perturbation in the lower troposphere, contrary to past observations and theory. The present study investigates the evolution of an asymmetric system and the causes for the unusual warm core structures. 


\section{TABLE OF CONTENTS}

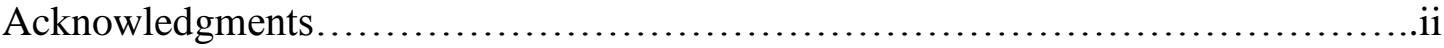

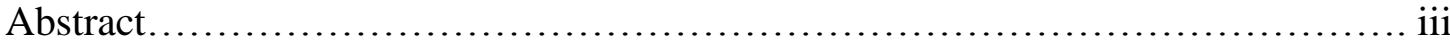

List of Tables...................................................................

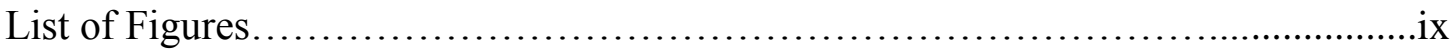

CHAPTER 1: INTRODUCTION ...........................................

1.1 A Need to Understand the Causes of Intensity Change........................1

1.2 Heat and Momentum Sources - their Impact on Intensity.....................2

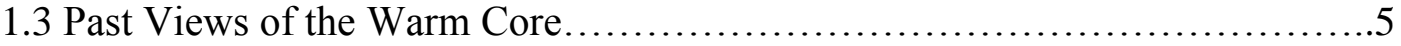

1.4 The Structure of Equivalent Potential Temperature in TCs....................7

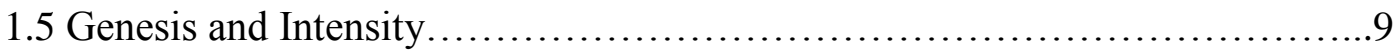

1.6 Goals....................................................................

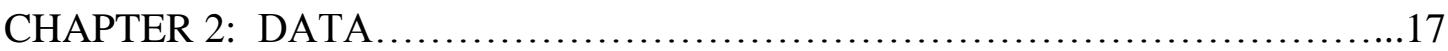

2.1 Flight Plan and Aircraft Involved in the Experiment..........................17

2.2 GPS Dropwindsonde Data.............................................. 17

2.2.1 Number of Dropwindsondes Deployed and their Distribution.............17

2.2.2 GPS Dropwindsonde Instrumentation...............................18

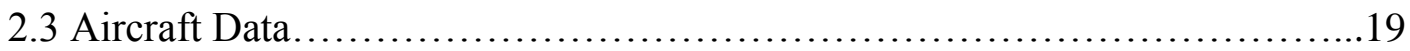

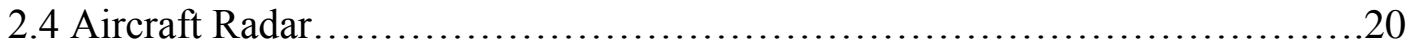

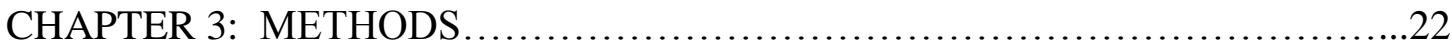

3.1 Processing GPS Dropwindsonde Data in ASPEN ............................22

3.1.1 Post Processing of GPS Dropwindsonde Data............................23 
3.1.2 Processing GPS Dropwindsonde Data.................................24

3.1.3 Calculating Storm Relative Winds and Thermodynamic Variables.........25

3.1.4 Missing Data and Interpolation Method..............................26

3.2 Testing Different Methods for Making Plan View Maps.......................26

3.3 Creating Three-Dimensional Matrices..................................... 31

3.3.1 Temperature Perturbation Vertical Cross-Sections.........................31

3.3.2 Calculation of Convective Available Potential Energy ...................32

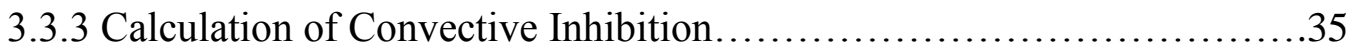

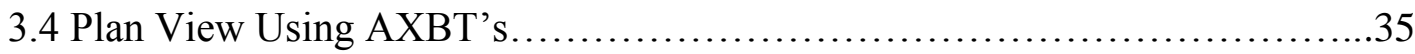

3.5 Calculation of Variables from Aircraft Data...................................35

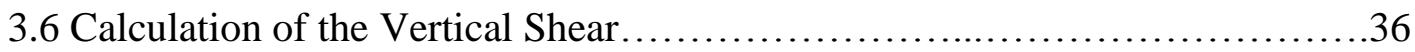

3.7 Energy Budget of Inflow................................................

3.7.1 Calculation of Divergence and Vertical Velocity.........................39

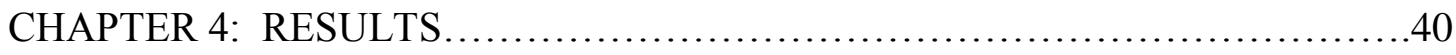

4.1 Large-Scale Environment................................................40

4.1.1 Formation of Humberto..............................................40

4.1.2 Evolution of Environmental Height Fields................................41

4.1.3 Evolution of Environmental Relative Humidity .........................41

4.1.4 Evolution of Sea Surface Temperatures..............................42

4.1.5 Evolution of the Wind Shear.........................................42

4.2 Tropical Storm Humberto: Clues to Formation.............................42

4.2.1 GPS Sonde Analysis and Reflectivity Structure.......................43

4.2.2 The Role of the Stratiform Precipitation in Forming the Warm Core......45 
4.2.3 Equivalent Potential Temperature Increase beneath the Nascent Warm

Core. .48

4.2.4 Evolution of Reflectivity Structures and Building an Eyewall............49

4.3 Energy Budget for the Warm $\theta_{\mathrm{e}}$ in the Eye.............................

4.3.1 Reflectivity Structure along the Inflow Path "B" .....................52

4.3.2 Low-Level Kinematic and Thermodynamic Structures................52

4.3.3 Surface Layer Flux Divergence................................53

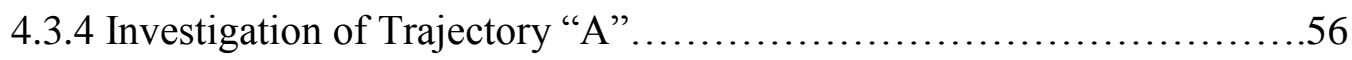

4.4 Storm-scale Structures during the Three Days of the Experiment and their

Relationship to the Environmental Wind Shear............................58

4.4.1 Evolution of Reflectivity Fields................................58

4.4.2 Comparison of Eyewall Radius..................................61

4.4.3 Low-level Kinematic Fields.....................................61

4.4.4 Divergence in the Boundary Layer...............................63

4.4.5 Low-level Equivalent Potential Temperature........................64

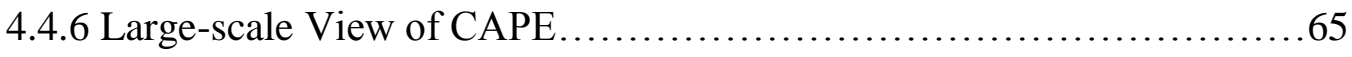

4.4.7 CAPE and CIN in the vicinity of the Eyewall.......................65

4.5 Thermodynamic Structures and their Relationship to Intensity..............66

4.5.1 Vertical Cross-sections of Equivalent Potential Temperature............66

4.5.2 Warm Core and Clues to Intensification..........................68

4.5.3 Asymmetric Structure of the Warm Core on the $23^{\text {rd }} \ldots \ldots \ldots \ldots \ldots \ldots . . . . .71$

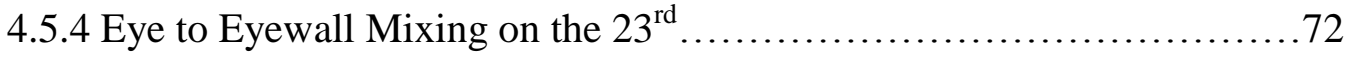

4.6 Environmental Influences on the Warm Core: Causes for the Unusual

Warm core Structures..........................................73 
4.6.1 Warm Core Structure and Dry air Intrusion on the $23^{\text {rd }} \ldots$ .73

4.6.2 Warm Core Structure and Dry Air Intrusion on the $24^{\text {th }} \ldots \ldots \ldots \ldots \ldots \ldots . .74$

4.7 The Complexity of Humberto: Contrasts to WISHE and Axisymmetry..........75

4.7.1 A Comparison of WISHE with Late Stage Genesis on the $22^{\text {nd }} \ldots \ldots \ldots . .75$

4.7.2 A Comparison of WISHE with Hurricane Humberto on the

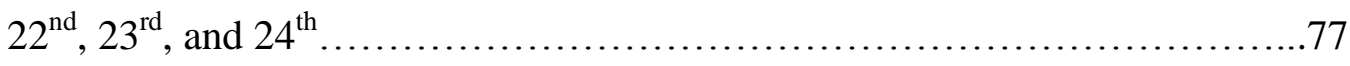

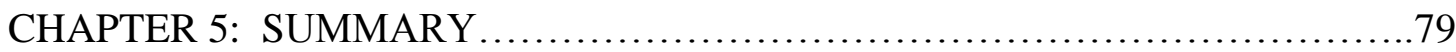

5.1 Conclusions........................................................ 79

5.2 Summary and Discussion.............................................. 81

5.2.1 Formation of Tropical Storm Humberto.............................81

5.2.2 Structure of Humberto in a High Vertical Wind Shear Environment......83

5.2.3 Thermodynamic Structures and the Intensity of Humberto..............84

5.2.4 Overall View of the Evolution of Humberto...........................86

5.3 Future Work.................................................... 87

REFERENCES ........................................................ 172 


\section{LIST OF TABLES}

$\underline{\text { Table }}$

$\underline{\text { Page }}$

1. Manufacturer's specifications for the GPS Dropwindsonde sensors.

Accuracy refers to the standard deviation of two successive calibrations

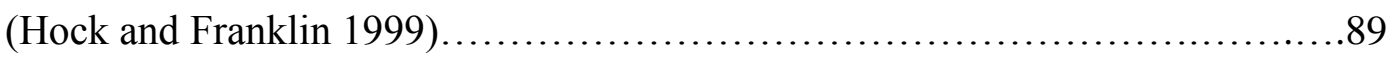

2. Instrumentation characteristics of NOAA WP-3D aircraft

(Jorgensen 1984a)................................................... 90

3. Airborne radar parameters for NOAA WP-3D aircraft (Jorgensen 1984a)........91 


\section{LIST OF FIGURES}

Figure $\quad$ Page

1. Annual average official intensity errors for Atlantic basin tropical cyclones for the period 1990-2005, with least-squares trend lines superimposed (National Hurricane Center) .................................................92

2. Vertical cross section of the temperature anomaly in Hurricane Hilda prepared from the aircraft and soundings (Hawkins and Rubsam 1968)

3. Vertical cross section of the temperature anomaly in Hurricane Inez prepared from the aircraft and soundings. Centers of anomalous warmth, located near the 600 and $300 \mathrm{mb}$ levels (Hawkins and

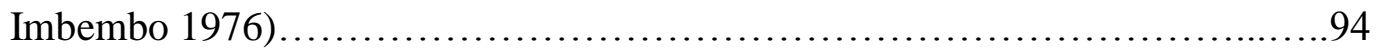

4. Composite cross section of mesoscale vertical velocity (w) computed by integrating the two-dimensional continuity equation and using the composite radial divergence analysis on 5 August 1980. Dark dashed line is radius of maximum wind (Jorgensen 1984b).

5. Vertical cross-section of equivalent potential temperature in Hurricane Inez Prepared from the aircraft and soundings (Hawkins and Imbembo 1976)..........96 
6. Radius-height cross-section for equivalent potential temperature $\theta_{\mathrm{e}}(\mathrm{K})$.

Regions where $\theta_{\mathrm{e}}>365$ are shaded. Heavy dashed line is RMW

(Jorgensen 1984b).

7. Vertical time section of the 24 hour changes of upper air temperature $\left({ }^{\circ} \mathrm{C}\right)$ at

Bikini. Dotted lines show mixing ratios of water vapor $\left(\mathrm{g} \mathrm{kg}^{-1}\right)$

(Yanai 1961).

8. Vertical time section of the 24 hour changes of upper air temperature $\left({ }^{\circ} \mathrm{C}\right)$ at

Kwajalein. Dotted lines show mixing ratios of water vapor $\left(\mathrm{g} \mathrm{kg}^{-1}\right)$

(Yanai 1961).

9. (A) Flight track the WP-3D on September $22^{\text {nd }} 2001$. (B) Same as "A"

except for the DC-8. Red star shows location of low-level circulation

center. Arrows indicate direction of the aircraft.

10. Flight tracks of the six aircraft observing Hurricane Humberto and its stratospheric, tropospheric, and upper-oceanic environment 23-24

September 2001. The red and black lines are the two NOAA

WP-3Ds; the blue line is the NASA DC-8; the violet line is the NASA

ER-2; and the green line is the NOAA G-IV (Aberson et al. 2006).

100

11. Location of NASA DC-8 and ER-2 dropwindsondes on September

$22^{\text {nd }}$. The four digits represent the last four digits of the

dropwindsonde number and the space to the left of the four digit

number is the location of the jettisoned dropwindsonde. Blue star

indicates storm circulation center.

101 
12. Location of WP-3D dropwindsondes on September $22^{\text {nd }}$. The four digits represent the last four digits of the dropwindsonde number and the space to the left of the four digit number is the location of the jettisoned dropwindsonde. Blue star indicates storm circulation center 102

13. Location of NASA DC-8 and ER-2 dropwindsondes on September $23^{\text {rd }}$. The four digits represent the last four digits of the dropwindsonde number and the space to the left of the four digit number is the location of the jettisoned dropwindsonde. Blue star indicates storm circulation center

14. Location of WP-3D (4 km flight level) dropwindsondes on September $23^{\text {rd }}$. The four digits represent the last four digits of the dropwindsonde number and the space to the left of the four digit number is the location of the jettisoned dropwindsonde. Blue star indicates storm circulation center 104

15. Location of WP-3D (1.5 km flight level) dropwindsondes on September $23^{\text {rd }}$. The four digits represent the last four digits of the dropwindsonde number and the space to the left of the four digit number is the location of the jettisoned dropwindsonde. Blue star indicates storm circulation center 105

16. GPS dropwindsonde and internal view (www.eol.ucar.edu ) 106 
17. Skew-T log-P diagram of sensor wetting correction. Red horizontal lines are pressure, blue lines slanted from bottom left to top right indicate temperature, dark blue lines slanted in opposite direction are dry adiabats, curved green lines are moist adiabats, blue dashed lines are water vapor, red line is sounding temperature, and blue dashed line is sounding dew point. Solid blue line shows where the dew point has been corrected

18. Same as Fig. 17 except Skew-T log-P showing radio frequency interference. Area from $660 \mathrm{hPa}$ to $750 \mathrm{hPa}$ was removed. 108

19. Plan view of the radial winds $\left(\mathrm{m} \mathrm{s}^{-1}\right)$ on the $23^{\text {rd }}$ of September, 2001, using the Barnes Objective Analysis. Numbers represent the exact values that each GPS sonde read. Color bar to the right denotes values given by the Barnes Objective Analysis .109

20. Plan view of the radial winds $\left(\mathrm{m} \mathrm{s}^{-1}\right)$ on the $23^{\text {rd }}$ of September, 2001, using the piecewise cubic Hermite interpolation. Numbers represent the exact values that each GPS sonde read. Color bar to the right denotes values given by the piecewise cubic Hermite interpolation 110

21. Plan view of the radial winds $\left(\mathrm{m} \mathrm{s}^{-1}\right)$ on the $23^{\text {rd }}$ of September, 2001. Analysis was done subjectively. Numbers represent the exact values that each GPS sonde read. Contours show values for subjective analysis.....

22. NCEP/NCAR Reanalysis $200 \mathrm{hPa}$ geopotential height field at $00 \mathrm{UTC}$ on September 22, 2001. Blue dot shows low-level circulation center. 
23. Track of Humberto from the time it became a tropical depression until it dissipated. Key shows Saffir-Simpson Scale

24. "Best Track" 1-minute sustained $10 \mathrm{~m}$ wind speed and surface pressure data for Humberto. Red line marks the pressure line with red diamonds showing the time of each observation. Blue line marks the $10 \mathrm{~m}$ wind speed with blue squares marking the time of each observation. Light yellow rectangles show times of observation for each of the three days

25. (A) NCEP/NCAR Reanalysis surface pressure chart (hPa) at 00 UTC on September $23^{\text {rd }}, 2001$. (B) NCEP/NCAR Reanalysis $500 \mathrm{hPa}$ geopotential height field with wind vectors. Time is 00 UTC on the $23^{\text {rd }}$ of September 2001

26. NCEP/NCAR Reanalysis $200 \mathrm{hPa}$ geopotential height field at $00 \mathrm{UTC}$ on September 23, 2001

27. Map of sea surface temperatures $\left({ }^{\circ} \mathrm{C}\right)$ from the AXBT's. AXBT's were deployed from approximately 18:30 - 24:00 UTC on the $22^{\text {nd }}, 23^{\text {rd }}$, and $24^{\text {th }}$ of September. Blue dots represent the circulation center and the associated time. Color bar to the right denotes sea surface temperature

28. The magnitude and direction of the vertical shear of the horizontal wind from the SHIPs model. Blue line shows the vertical wind shear magnitude $\left(\mathrm{m} \mathrm{s}^{-1}\right)$. Red line displays the shear direction. Light yellow rectangles show times of observation for each of the three days 
29. Time-space composite of GPS sondes. Latitude and longitude delineated every 0.5 degrees. Pressure ( $\mathrm{hPa}$ ) is in color and streamlines are shown. Color bar to the right denotes pressure. White dot marks circulation center. Four digit numbers correspond to GPS sonde locations

30. Plan view reflectivity image at 19:00 UTC on September $22^{\text {nd }}$. Image is $120 \times 120 \mathrm{~km}$. White dot shows the center of low pressure. Lines A and $\mathrm{B}$ are RHI reflectivity images shown in the next two figures. Two black dots show the position of the WP-3D at the time of the RHI scans. The WP-3D is moving east northeast, just north of the low-level circulation Center.

31. (A) RHI reflectivity image at $18: 53$ UTC on September $22^{\text {nd }}$, 2001. Image is $120 \mathrm{~km}$ wide and $20 \mathrm{~km}$ high. Letter " $\mathrm{A}$ " corresponds to the line in figure 27. (B) Same as "A" except time is 18:54 UTC. "B" corresponds to the line in Figure 27

32. Streamlines at $5 \mathrm{~km}$ height as a function of latitude and longitude (degrees). Red dot corresponds to low-level pressure center

33. Schematic cross section through a class of squall system. All flow is relative to the squall line which is moving from right to left. Circled numbers are typical values of $\theta_{\mathrm{w}}$ in ${ }^{\circ} \mathrm{C}$ (Zipser 1977) 
34. Skew-T log-p diagram of calculated profiles of temperature and dew point for mesoscale downdrafts originating at $600 \mathrm{mb}$ with a temperature of $0^{\circ} \mathrm{C}$, a relative humidity of $100 \%$, and vertical velocities of $0.2 \mathrm{~m} \mathrm{~s}^{-1}$ (heavy solid lines) and $0.5 \mathrm{~m} \mathrm{~s}^{-1}$ (heavy dashed lines) superposed on Zipser's (1977, Fig. 8) collection of soundings beneath anvil clouds. Vertical coordinate is pressure and all temperatures are in ${ }^{\circ} \mathrm{C}$. (Leary 1980)......................... 124

35. Same as Fig. 17. GPS sonde jettisoned at 18:54 UTC on September $22^{\text {nd }}$, from the DC- 8 , near the surface circulation center. 125

36. Same as Fig. 17. GPS sonde jettisoned at 21:19 UTC on September $22^{\text {nd }}$, from the ER-2, near the surface circulation center.

37. Vertical velocity $\left(\mathrm{m} \mathrm{s}^{-1}\right)$ as a function of time (UTC) from the WP-3D flying at $5 \mathrm{~km}$ 127

38. East-west vertical cross-section of the temperature anomaly, on an axis through the surface circulation center. Color bar indicates the temperature anomaly in degrees Kelvin. Storm center is located at $66.7^{\circ} \mathrm{W} \ldots \ldots 128$

39. Plan view of temperature at $400 \mathrm{hPa}$ in degrees Celsius from the GPS sondes. Temperature contoured (solid red) every degree. Black circle marks low-level circulation center. 
40. Plan view at 20 meter height of equivalent potential temperature. Streamlines superposed as blue lines with arrows. Latitude and longitude delineated every 0.5 degrees. Color bar at right corresponds to equivalent potential temperature (Kelvin). White dot indicate surface circulation center.....130

41. Vertical cross-section of equivalent potential temperature, taken $20^{\circ}$ clockwise of a north-south axis, through the surface circulation Center. Color bar to right corresponds to equivalent potential temperature (Kelvin). Low-level circulation center is located at $28.9^{\circ} \mathrm{N}$

42. Radial scatterplots of equivalent potential temperature $(\mathrm{K})$ calculated from the U.S. Air Force reconnaissance flight data with 30-s time resolution. All values are from elevations between 200 and $500 \mathrm{~m}$ : (a) 1600-2300 UTC 16 Jul, during depression stage; (b) 1400-2000 UTC $17 \mathrm{Jul}$, during early tropical storm stage when there were multiple vortex interactions; (c) 2200 UTC 17 Jul-0600 UTC 18 Jul, during late tropical storm stage; and (d) 1100-2100 UTC 18 Jul, the first full flight at hurricane intensity. The vertical dashed line indicates the radius of maximum wind taken from an average of the four-six estimates during each flight by reconnaissance aircraft. The value in (c) is averaged from the flights on either side. The horizontal line lies at $\theta_{\mathrm{e}}=355 \mathrm{~K}$.

(Molinari et al. 2004)

43. Plan view of CAPE $\left(\mathrm{J} \mathrm{kg}^{-1}\right)$ and CIN $\left(\mathrm{J} \mathrm{kg}^{-1}\right)$. Latitude and longitude delineated every 0.5 degrees. CAPE is shown by the values on the color bar. CIN is represented by the contours. Circulation center is marked by a blue " $\mathrm{x}$ " 
44. Plan view reflectivity image at 19:42 UTC on September $22^{\text {nd }}$. Image is $120 \times 120 \mathrm{~km}$. White dot shows the low-level circulation center. Location of the WP-3D is marked by the white plus symbol in the center of the image

45. Equivalent potential temperature as measured from the NOAA WP-3D from 19:38 UTC through 19:47 UTC on 9/22/01. Y axis is the equivalent potential temperature in degrees Kelvin and the $\mathrm{x}$ axis is time

46. Plan view reflectivity image at 21:09 UTC on September $22^{\text {nd }}$. Image is 240 x $240 \mathrm{~km}$. White dot shows the center of low pressure...

47. Plan view reflectivity image at 21:23 UTC on September $22^{\text {nd }}$. Image is $240 \times 240 \mathrm{~km}$. White dot shows the center of low pressure

48. Schematic showing the three trajectories. The black dashed line depicts the $25 \mathrm{~km}$ radius from the center. " $\mathrm{A}$ ", " $\mathrm{B}$ ", and " $\mathrm{C}$ " mark the 3 trajectories. The point marked "decision point" displays where trajectory "B" splits. "B2" is the first trajectory analyzed. "B1" displays the new trajectory based on the results of the first energy budget 138

49. Plan view reflectivity image at $18: 58$ UTC on September $22^{\text {nd }}$. Image is $240 \times 240 \mathrm{~km}$. Black dot shows the center of lowest surface pressure and black dashed line indicates the inflow trajectory .139 
50. Plan view of $\theta_{\mathrm{e}}$ at 10 meters. Solid contours denote equivalent potential temperature every $5 \mathrm{~K}$. Inflow trajectory is the black dashed line and the black "X" marks the positions of the 3 soundings. Red spot is the low-level circulation center.

51. 10 meter plan view of earth relative total wind speed. Contours denote wind speed every $2 \mathrm{~m} \mathrm{~s}^{-1}$. Inflow trajectory is the black dashed line and the black " $\mathrm{X}$ " marks the positions of the 3 soundings. Black circle is the low-level circulation center.

52. Plan view of the 10 meter temperature in degrees Celsius. Contours denote temperature every $1^{\circ} \mathrm{C}$. Inflow trajectory is the black dashed line and the black " $\mathrm{X}$ " marks the positions of the 3 soundings. Black circle is the low-level circulation center

53. Plan view of the 10 meter specific humidity in $\mathrm{g} \mathrm{kg}^{-1}$. Contours denote specific humidity every $1 \mathrm{~g} \mathrm{~kg}^{-1}$. Inflow trajectory is the black dashed line and the black " $\mathrm{X}$ " marks the positions of the 3 soundings. Black circle is the low-level circulation center.

54. Vertical cross-section of divergence $\left(\mathrm{s}^{-1}\right)$ calculated from sounding 1 to 2 (refer to Fig. 50). 
55. Vertical profiles of $\theta_{\mathrm{e}}$ soundings along the inflow path "B2".

Soundings 1, 2, and 3 are from Fig 50. Dashed line displays how the mixed layer rises in the presence of convergence............................. 145

56. The initial sounding marked "I" and final sounding marked "F" for trajectory "A" shown in Fig. 48. Yellow displays amount of $\theta_{\mathrm{e}}$ gained with the divergence adjusted sounding.

57. Nine lower fuselage scans $(240 \mathrm{~km} \times 240 \mathrm{~km})$ of evolution of eyewall.

Times to the nearest minute and dates are (A) 19:00 UTC 9/22/01

(B) 19:38 UTC 9/22/01 (C) 21:23 UTC 9/22/01 (D) 20:26 UTC 9/23/01

(E) 22:59 UTC 9/23/01 (F) 24:30 UTC 9/23/01 (G) 21:27 UTC 9/24/01

(H) 22:59 UTC 9/24/01 (I) 24:30 UTC 9/24/01. Color scale on right indicates $\mathrm{dBZ}$ values.

58. Six lower fuselage scans $(120 \mathrm{~km} \times 20 \mathrm{~km})$ of evolution of eyewall. Times to the nearest minute and dates are (A) 18:53 UTC 9/22/01

(B) 20:02 UTC 9/22/01 (C) 23:28 UTC 9/23/01 (D) 23:26 UTC 9/23/01

(E) 21:28 UTC 9/24/01 (F) 24:28 UTC 9/24/01. Color scale on bottom indicates $\mathrm{dBZ}$ values. .148 
59. Plan views of the tangential winds $\left(\mathrm{m} \mathrm{s}^{-1}\right)$ at $50 \mathrm{~m}$ and $1.5 \mathrm{~km}$ for various days of the experiment. Color bar to right delineates the magnitude $\left(\mathrm{m} \mathrm{s}^{-1}\right)$. Note difference in horizontal scale for each day. (A) $50 \mathrm{~m}$ on 9/22/01

(B) $1.5 \mathrm{~km}$ on $9 / 22 / 01$ (C) $50 \mathrm{~m}$ on $9 / 23 / 01$ (D) $1.5 \mathrm{~km}$ on $9 / 23 / 01$

(E) $50 \mathrm{~km}$ on $9 / 24 / 01(\mathrm{~F}) 1.5 \mathrm{~km}$ on $9 / 24 / 01$.

60. Plan views of the radial winds $\left(\mathrm{m} \mathrm{s}^{-1}\right)$ at $50 \mathrm{~m}$ and $1.5 \mathrm{~km}$ for various days of the experiment. Color bar to right delineates the magnitude $\left(\mathrm{m} \mathrm{s}^{-1}\right)$. Note difference in horizontal scale for each day. (A) $50 \mathrm{~m}$ on $9 / 22 / 01$

(B) $1.5 \mathrm{~km}$ on $9 / 22 / 01$ (C) $50 \mathrm{~m}$ on $9 / 23 / 01$ (D) $1.5 \mathrm{~km}$ on $9 / 23 / 01$

(E) $50 \mathrm{~km}$ on $9 / 24 / 01(\mathrm{~F}) 1.5 \mathrm{~km}$ on $9 / 24 / 01$. .150

61. Divergence $\left(\mathrm{s}^{-1}\right)$ calculated at $200 \mathrm{~m}$ from just outside the eyewall to the circulation center. Circle size is a function of eyewall radius. (A) 9/22/01 (B) $9 / 23 / 01$ (C) $9 / 24 / 01$

62. Plan views of $\theta_{\mathrm{e}}$ for various days of the experiment at $200 \mathrm{~m}$ and $3 \mathrm{~km}$. Color bar to right delineates the magnitude (Kelvin). Note difference in horizontal scale for day. (A) $200 \mathrm{~m}$ on $9 / 22 / 01$ (B) $3 \mathrm{~km}$ on $9 / 22 / 01$ (C) $200 \mathrm{~m}$ on $9 / 23 / 01$ (D) $3 \mathrm{~km}$ on $9 / 23 / 01$ (E) $200 \mathrm{~km}$ on $9 / 24 / 01$ (F) $3 \mathrm{~km}$ on $9 / 24 / 01$. The white rings show the location of the eyewall based on the reflectivity 152 
63. Large-scale plan view of CAPE and streamlines at 50 meters. Color bar shows values of CAPE in $\mathrm{J} \mathrm{kg}^{-1}$. Note difference in horizontal scales for each day. (A) 9/22/01 (B) 9/23/01 (C) 9/23/01.

64. $200 \mathrm{~km} \times 200 \mathrm{~km}$ plan views of convective available potential energy $\left(\mathrm{J} \mathrm{kg}^{-1}\right)(\mathrm{CAPE})$, convective inhibition $\left(\mathrm{J} \mathrm{kg}^{-1}\right)(\mathrm{CIN})$, and streamlines. Latitude and longitude delineated every 0.5 degrees. White and black circles are approximate location of the RMW from the low-level WP-3D (A) CAPE on $9 / 23 / 01$ (B) CIN on $9 / 23 / 01$ 154

65. $200 \mathrm{~km}$ x $200 \mathrm{~km}$ plan views of convective available potential energy $\left(\mathrm{J} \mathrm{kg}^{-1}\right)(\mathrm{CAPE})$, convective inhibition $\left(\mathrm{J} \mathrm{kg}^{-1}\right)(\mathrm{CIN})$, and streamlines. Latitude and longitude delineated every 0.5 degrees. White and black circles are approximate location of the RMW from the low-level WP-3D (A) CAPE on $9 / 24 / 01$ (B) CIN on $9 / 24 / 01$ 155

66. Vertical cross-sections of $\theta_{\mathrm{e}}$ (Kelvin). (A) $160 \mathrm{~km} \mathrm{x} 3 \mathrm{~km}$ on 9/22/01. Southern eyewall is located at $28.6^{\circ} \mathrm{N}$. Northern eyewall is located at $29.1^{\circ} \mathrm{N}$ (B) $200 \mathrm{~km} \times 3.4 \mathrm{~km}$ on $9 / 23 / 01$. Southern eyewall is located at $32.3^{\circ} \mathrm{N}$. Northern eyewall is located at $32.8^{\circ} \mathrm{N}$. (C) $200 \mathrm{~km} \times 3.4 \mathrm{~km}$ on $9 / 24 / 01$ 156

67. Vertical cross-sections of the warm core anomaly of Humberto in degrees Kelvin for (A) 9/22/01 (B) 9/23/01 (C) 9/24/01 157 
68. Pressure perturbation in $\mathrm{hPa}$ for $1 \mathrm{~km}$ layers from 0 to $10 \mathrm{~km}$.
(A) $9 / 22 / 01$ (B) $9 / 23 / 01$ (C) $9 / 24 / 01$
158

69. The evolution of the warm core as a function of height and day from the $22^{\text {nd }}$ to the $24^{\text {th }}$. Contours are of the warm core anomaly every 1 degree Kelvin

70. North to south vertical cross-section of the temperature perturbation (Kelvin) for Humberto on the $23^{\text {rd }}$. Color bar to right shows values in degrees Kelvin. Line "A" represents the latitudinal position of a GPS sonde jettisoned on the inner edge of the northern eyewall. Line "B" represents the latitudinal position of a GPS sonde jettisoned in the southern RMW. Circulation center is at $32.5^{\circ} \mathrm{N}$

71. All soundings plotted on a skew-T log-P diagram. (A) Sounding taken at the position of line "A" from Fig. 70, jettisoned from the WP-3D.

(B) Sounding taken at the position of line "B" from

Fig. 70, jettisoned from the WP-3D. (C) Sounding taken just west of the sounding "B" in Fig. 70, jettisoned from the WP-3D.

72. Close up plan view of $\theta_{\mathrm{e}}$ at $200 \mathrm{~m}$ and streamlines on $9 / 23 / 01$. Color bar to right delineates magnitude (Kelvin) Grey dots show where the radius of maximum wind is located according to the low-level WP-3D 162 
73. (A) South to north view of the vertical velocity, tangential winds, and equivalent potential temperature taken from the WP-3D flying at $1.8 \mathrm{~km}$ on 9/23/01. (B) Southeast to northwest view taken from the WP-3D flying at $5 \mathrm{~km}$ on $9 / 23 / 01$. .163

74. Vertical cross-section from north to south of the relative humidity on 9/23/01 from 0-10 km. Color bar at right corresponds to the relative humidity. Eye is located at $32.5^{\circ} \mathrm{N}$. Southern eyewall located at $32.2^{\circ} \mathrm{N}$. Northern eyewall is located at $32.8^{\circ} \mathrm{N}$ 164

75. Schematic of the effect of dry air on an undiluted updraft containing CAPE. Pressure is on the y-axis and temperature is along the $\mathrm{x}$-axis. Red line is undiluted updraft. Black line is an environmental sounding representing a moist adiabat. Yellow area is CAPE. Blue hatched area is cooling due to dry air intrusion. .165

76. Vertical cross-section from north to south of the relative humidity on 9/24/01 from 0-10 km. Color bar at right corresponds to the relative humidity. The eye is located at $36.4^{\circ} \mathrm{N}$. Southern eyewall is located at $36^{\circ} \mathrm{N}$. Northern eyewall is located at $36.8^{\circ} \mathrm{N}$ 166

77. Schematic of the difference in the temperature perturbation between 9/24/01 and 9/23/01. Contours are every 1 degree Kelvin. .167 
78. Comparison of Humberto temperature anomaly (green) with WISHE temperature anomaly (red). (A) 9/22/01 (B) 9/23/01 (C) 9/24/01. Red dashed line on $9 / 24 / 01$ is the WISHE temperature anomaly if the eye $\theta_{\mathrm{e}}$

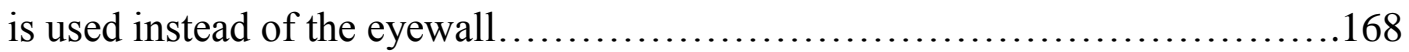




\section{Appendix}

1. NCEP/NCAR Reanalysis displaying divergence $\left(\mathrm{s}^{-1}\right)$ at $200 \mathrm{hPa}$ geopotential at 00 UTC on September 22, 2001. Black dot shows low-level

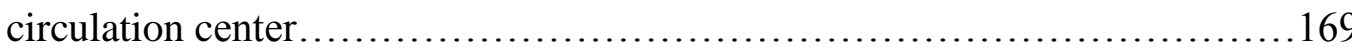

2. North to south vertical cross-section of the radial winds $\left(\mathrm{m} \mathrm{s}^{-1}\right)$ on $9 / 23 / 01$ Color bar on right denotes the radial wind velocity $\left(\mathrm{m} \mathrm{s}^{-1}\right)$ 170

3. Plan views of relative humidity at 2 and $7 \mathrm{~km}$ for various days of the experiment. Color bar to right delineates the relative humidity. Note difference in horizontal scales for each day. (A) $2 \mathrm{~km} \mathrm{9/22/01}$

(B) $7 \mathrm{~km} \mathrm{9/22/01} \mathrm{(C)} 2 \mathrm{~km} \mathrm{9/23/01} \mathrm{(D)} 7 \mathrm{~km} \mathrm{9/23/01} \mathrm{(E)} 2 \mathrm{~km} \mathrm{9/24/01}$

(F) $7 \mathrm{~km} \mathrm{9/24/01}$ 


\section{Chapter 1}

\section{Introduction}

\subsection{A Need to Understand the Causes of Intensity Change}

The identification of the causes of intensity change of tropical cyclones (TCs) has remained a major challenge for the meteorological community (Marks et al. 1998, AMS statement on Hurricane Research and Forecasting 2006, National Science Board Report 2006, Holland and Lukas 2006). Intensity change may be defined as either a deepening or filling of central sea-level pressure or an increase or decrease of the sustained wind speeds in the eyewall. The forecast intensity errors for the Atlantic Basin (Fig.1) show that the errors constitute about a full category on the Saffir-Simpson Scale beyond 48 hours. This is evidence that our forecasting ability has not evolved substantially in the last 15 years.

A contributing factor to our lack of understanding about intensity variations is the inadequate sampling of a TC throughout its lifecycle. In 2001 the National Oceanic and Atmospheric Administration (NOAA) and the National Aeronautical and Space Administration (NASA) marshaled their resources to collect a more complete dataset that may be utilized to address the evolutionary aspects of a TC. The unprecedented sampling of TC Humberto during the Convection and Moisture Experiment (CAMEX-4) provides an opportunity to examine the details of changes in intensity as Tropical Storm Humberto intensifies to a category 2 hurricane and then weakens to a category 1 . Rarely do we get a view of storm evolution over 3 successive days with multiple aircraft, the deployment 
of over 200 Global Positioning System dropwindsondes (GPS sondes), and airborne expendable bathythermographs (AXBTs).

Frank (1977) successfully identified the synoptic-scale features of a mature typhoon by compositing the observations from hundreds of TCs. Instead of gathering many storms of different intensity and spatial scale, in this study I examine the evolution of an individual storm as it progresses through the early stages of development. Using methods similar to those of Frank (1977), I composite GPS sondes jettisoned over 3 to 4 hours for each day of the experiment as Humberto evolves.

The observations of Hurricane Humberto provide a quantitative assessment of the structure of a storm that forms, intensifies, and weakens at higher latitudes. During the peak intensity and the period of filling, Humberto is in a high shear environment, traveling over large sea surface temperature (SST) gradients. A comparison of the structures in Humberto with past observations of more intense low latitude storms, developing in a more homogeneous environment, reveal important structural differences between these two types of systems.

\subsection{Heat and Momentum Sources - their Impact on Intensity}

Theoreticians investigate intensity changes based on idealized models. Shapiro and Willoughby (1982) and Holland and Merrill (1984) examined the response of a hurricane-like vortex to imposed heat and momentum sources via the Sawyer-Eliassen equation. A heat or momentum source is placed at various locations in an axisymmetric (two-dimensional) vortex initially in gradient balance to see how the TC structure responds. The response is not only a function of the chosen magnitude and location of the sources but also a function of the inertial and static stabilities of the vortex. Inertial 
stability is controlled by the horizontal gradient of the absolute angular momentum surfaces while static stability is a function of the vertical gradient of the Brunt-Vaisala frequency.

A heat source placed in an area of high inertial stability can have two effects; one is to increase the secondary circulation of the $\mathrm{TC}$, another is to cause dry adiabatic descent on the inner edge of the eyewall. Shapiro and Willoughby (1982) show that this can cause a pressure drop on the inner edge of the eyewall and in the eye which causes both a decrease in eyewall radius and the hydrostatic pressure in the eye.

Momentum sources and sinks are another possible source of intensity change. Momentum sources tend to have larger effects at higher altitudes because of the low inertial stability found there and are therefore capable of changing the intensity of a TC.

Although the aforementioned theoretical model is far removed from a real hurricane, a number of observed variables can be grouped into heat and momentum sources. Higher amounts of mid-level moisture, convective outbreaks, eye to eyewall mixing, warmer SST, and cooler outflow temperatures associated with a cooler tropopause are believed to promote greater intensity and may also be viewed as heat sources (Malkus and Riehl 1960, Fletcher et al. 1961, Miller 1964, Gray 1968, Jorgensen 1984, Black 1986, Emanuel 1986, Franklin et al. 1988, Emanuel 1989, Molinari and Vollaro 1989, DeMaria and Kaplan 1994, 1999, DeMaria 1996, Bosart et al. 2000, Hanley et al. 2001, Persing et al. 2002, Zhang et al. 2002, Eastin et al. 2005, Heymsfield et al. 2006, Montgomery et al. 2006, Schubert et al. 2006). Any of the previously mentioned variables may increase the instability of the eyewall and may induce subsidence in the eye. Rainbands can play an important role by altering the energy 
content in the boundary layer (BL) (Barnes et al. 1983, Powell 1990) and act to reduce the energy content available to the eyewall. A detailed examination of radial flows above and in the BL along with thermodynamic conditions in the BL and eyewall provide an opportunity to investigate whether or not Humberto shares characteristics with this idealized model.

Upper level outflow jets, vertical shear of the horizontal wind, changes in the radius of maximum wind (RMW), low level momentum surges, and Vortex Rossby Waves (VRWs) are all possible momentum sources (Sadler 1978, Lewis and Jorgensen 1978, Holland and Merrill 1984, Molinari et al. 1995, Montgomery and Lu 1997, Moller and Montgomery 1999, Bosart et al. 2000, Ventham and Wang 2007). Radar data will be analyzed to track changes in the eyewall radius. Through conservation of angular momentum, these changes may be linked to intensity changes. Vertical wind shear is calculated from the statistical hurricane intensity prediction scheme (SHIPS) model (DeMaria and Kaplan 1999, DeMaria et al. 2005). Structural changes in the wind fields from day to day may identify whether momentum surges at lower levels have an effect on intensity.

WISHE (wind induced surface heat exchange) theory is related to the increase of BL energy content and is currently the most accepted theory of intensification. In WISHE the intensification of a hurricane depends on the fluxes at the air-sea interface to increase the equivalent potential temperature $\left(\theta_{\mathrm{e}}\right)$ of the BL air. As a parcel with warmer $\theta_{\mathrm{e}}$ reaches the eyewall, it will ascend along a warmer moist adiabat than the previous parcel. Moist adiabats diverge with height resulting in the greatest temperature differences between the eyewall and the environment in the upper troposphere (Emanuel 
1986). Observations of mature TCs (Hawkins and Rubsam 1968) show that the maximum temperature perturbation exists in the upper troposphere, consistent with the WISHE theory. WISHE produces a slight instability in the eyewall where inertial stability is high. This may be viewed as a heat source although the heat source produces small vertical velocities in the model and does not produce a secondary circulation in the eye as displayed by Shapiro and Willoughby (1982) and Holland and Merrill (1984). Whether or not the warm core is an artifact of diverging moist adiabats (WISHE) or subsidence due to a compensating secondary circulation in the eye is still an issue that needs clarification.

The present study offers views of the warm core in the early stages of development through the depth of the troposphere. Vertical cross-sections of the warm core in Humberto are compared to what would be expected if the WISHE theory was responsible for maintaining the warm core and with the theoretical constructs of Shapiro and Willoughby (1982) and Holland and Merrill (1984).

\subsection{Past Views of the Warm Core}

In 1968 the first vertical cross-section of the temperature perturbation of a mature hurricane over water was published (Hawkins and Rubsam 1968). Hurricane Hilda reached a minimum central pressure of $941 \mathrm{hPa}$ just after the research flights were made. As seen in Fig. 2, TC Hilda had a maximum temperature perturbation of $16 \mathrm{~K}$ near 300 hPa (Hawkins and Rubsam 1968).

Hawkins and Imbembo (1976) used similar methods as those used to investigate the vertical structure of Hilda in a smaller, more intense TC Inez. The first passes through Inez were made in the early morning on 9/27, while Inez was intensifying. The 
warm core (Fig. 3) showed two distinct areas of increased temperature perturbation, one at $300 \mathrm{hPa}$ and one at $600 \mathrm{hPa}$. Both of these centers of anomalous high temperature are about $9 \mathrm{~K}$ above the mean tropical atmosphere. These perturbations were calculated by comparing them to the Jordan (1958) mean tropical sounding. When research planes flew back into the storm on the morning of $9 / 28$ the minimum central pressure of Inez dropped to $927 \mathrm{hPa}$ with maximum wind speeds of $78 \mathrm{~m} \mathrm{~s}^{-1}$ Temperature maxima were detected at $250 \mathrm{hPa}(16 \mathrm{~K}$ perturbation) and $600 \mathrm{hPa}(11 \mathrm{~K})$.

Two-thirds of the vertical cross-sections discussed above display anomalous areas of warming in the mid-troposphere. The structures of these TCs are inconsistent with some aspects of the WISHE theory. WISHE theory only explains the maximum temperature perturbation in the upper troposphere due to the transport of warm air in the eyewall. The theory does not account for anomalous centers of warmth in the midtroposphere. This suggests that other processes might be involved in maintaining or intensifying the warm core. Humberto offers the opportunity to view the structure of the warm core of a TC in the early stages of development. Vertical cross-sections of the temperature perturbation in a tropical storm, category 1, and category $2 \mathrm{TC}$ have not been shown before.

Jorgensen (1984b) was the first to show the evolution of the eye and eyewall of a TC. The August $5^{\text {th }}$ flights were the first time that coordinated, repetitive legs were flown in a specific region of the storm at five altitudes. These flights show the structure at four different times. The largest updraft in Fig. 4 occurs about one km inside the RMW. This is an area that has very high inertial stability, so motions tend to be confined to the vertical. About $15 \mathrm{~km}$ radially inward from the RMW is a region of air descending at 
about -2 to $-3 \mathrm{~m} \mathrm{~s}^{-1}$. An unsaturated downdraft causes additional warming and hydrostatic pressure falls on the inner edge of the eyewall. Although Jorgensen (1984b) doesn't show a vertical cross section of the warm core, his analysis does hint that a secondary circulation in the eye may be playing a role in warming the atmosphere.

\subsection{The Structure of Equivalent Potential Temperature in TCs}

Equivalent potential temperature is related to the total energy content of an air parcel. It is useful in that it is a conserved variable in the absence of energy gains from the sea surface and energy losses from radiation which are generally small over the typical composite time. Equivalent potential temperature has been linked to intensity through both empirical and theoretical arguments (Malkus and Riehl 1960, Emanuel 1986). A surge of higher $\theta_{\mathrm{e}}$ into the eyewall may also cause larger vertical accelerations and compensating secondary circulation in the eye similar to theoretical constructs put forth by Shapiro and Willoughby (1982) and Holland and Merrill (1984).

Hawkins and Imbembo (1976) used multiple aircraft to construct a cross-section of $\theta_{\mathrm{e}}$ in hurricane Inez when it was a $927 \mathrm{hPa}$ storm (Fig. 5). High values and tight gradients of $\theta_{\mathrm{e}}$ are found in the eyewall region from the surface to the tropopause. Figure 5 does show that values of $\theta_{\mathrm{e}}$ increase in the BL as you approach the eyewall region where wind speeds increase markedly. Figure 5 also displays very high values of $\theta_{\mathrm{e}}$ in the lower eye region with values of $366 \mathrm{~K}$. Surface data are inferred from aircraft observations at higher levels. The exact location of the tight radial gradient of $\theta_{\mathrm{e}}$ in the BL with respect to the RMW is questionable. Does this gradient of $\theta_{\mathrm{e}}$ occur at radii just outside the eyewall and underneath the eyewall or does this gradient exist from the inner 
edge of the eyewall into the eye? Understanding where $\theta_{\mathrm{e}}$ increases in relation to convection in the eyewall is vital to intensity forecasts.

Jorgensen (1984b) also investigated the thermodynamic structure from the center of circulation to $120 \mathrm{~km}$ radial distance. In Fig. $6 \theta_{\mathrm{e}}$ increases from $355 \mathrm{~K}$ at a $100 \mathrm{~km}$ radius and a height of about $1.5 \mathrm{~km}$ to a value of $365 \mathrm{~K}$ at the RMW. That is about a 10 $\mathrm{K}$ increase in $70 \mathrm{~km}$. From the RMW to about $10 \mathrm{~km}$ from the storm center, $\theta_{\mathrm{e}}$ increased from $365 \mathrm{~K}$ to about $375 \mathrm{~K}$ at a $1.5 \mathrm{~km}$ height. That is an increase of $10 \mathrm{~K}$ in approximately $15 \mathrm{~km}$. Other passes show similar results with pass 3 displaying an increase of $15 \mathrm{~K}$ for a similar $20 \mathrm{~km}$ distance radially inward from the RMW and a $10 \mathrm{~K}$ increase from the RMW to a radius of $110 \mathrm{~km}$ ( $70 \mathrm{~km}$ from the RMW). Figure 6 also shows that the $375 \mathrm{~K}$ air goes up to about $3 \mathrm{~km}$ while the $370 \mathrm{~K}$ air gets as high as $5 \mathrm{~km}$ inside the eye of the storm. The air inside the eye is thought to be moving in solid body rotation, so wind speeds near the center are quite slow. The low wind speeds inside the eye should only allow for higher values of $\theta_{\mathrm{e}}$ if the air has a long residence time (Barnes and Fuentes 2010). Jorgensen (1984b) is showing a competing example of $\theta_{\mathrm{e}}$ profiles where there is a steady increase in $\theta_{\mathrm{e}}$ moving radially in towards the RMW and then the tight gradient occurs within the RMW. Both of the cross-sections by Hawkins and Imbembo (1976) and Jorgensen (1984b) do not have data in the BL. Where the tight gradient of $\theta_{\mathrm{e}}$ exists in relation to the eyewall still needs clarification. The distribution of $\theta_{\mathrm{e}}$ might also change depending on whether the storm is intensifying or weakening and if the environmental conditions in which it is embedded evolve.

In Humberto, plan views and vertical cross-sections of $\theta_{\mathrm{e}}$ are made from the midtroposphere down to the sea surface. These images help to better define the distribution 
of $\theta_{\mathrm{e}}$ in the BL. The previous studies mentioned had to extrapolate $\theta_{\mathrm{e}}$ from the lowest flight level, making the location of high $\theta_{\mathrm{e}}$ gradients near the eyewall questionable. Mapping the location of the RMW in concert with detailed maps of $\theta_{\mathrm{e}}$ in the BL help to clarify exactly where large gradients of $\theta_{\mathrm{e}}$ exist. Past research indicates that fluxes of sensible and latent heat from the ocean surface increase greatly near the eyewall in response to higher wind speeds (Riehl and Malkus 1961, Hawkins and Rubsam 1968, Wroe and Barnes 2003). The Humberto data will help identify if $\theta_{\mathrm{e}}$ increases occur at further distances from the eye and if this air is advected into eyewall or if the increases occur near and under the eyewall. There might also be evidence for higher $\theta_{\mathrm{e}}$ in the eye mixing into eyewall.

\subsection{Genesis and Intensity}

Intensity change may be defined as a fluctuation in the intensity of a TC through its entire life, including the early stages of TC development. I will be defining genesis as the time at which the warm core develops. There is much debate about how the warm core first forms.

Besides the environmental factors outlined by Gray $(1968,1975,1979)$ genesis requires a pre-existing disturbance. Although most of the early studies on genesis highlight the importance of a favorable large-scale environment (Hubert 1955, Riehl 1948, McBride and Zehr 1981), these studies are not able to resolve the inner $100 \mathrm{~km}$ of developing systems. Yanai (1961) states that the warm core occurs first at $300 \mathrm{hPa}$ and that the warming gradually spread downward. Plots of the temperature perturbation as a function of time from rawinsonde stations in the Marshall Islands as typhoon Doris is forming are shown in Figs. 7 and 8. At Bikini (Fig. 7) warming of about $2^{\circ} \mathrm{C}$ is located at 
the 300-500 hPa level just above the low-level trough axis (dark black lines in Figs. 7 and 8). Maximum warming of $4^{\circ} \mathrm{C}$ is found at $100 \mathrm{hPa}$ at Kwajalein (Fig. 8) at the upper level trough axis and there is cooling of $-5^{\circ} \mathrm{C}$ above the low level trough axis. A major problem with this analysis is that Bikini is located more than $400 \mathrm{~km}$ north of the lowlevel circulation center and Kwajalein is located about $200 \mathrm{~km}$ north of the circulation center.

Conditional Instability of the Second Kind (CISK) has formed the basis of much of the theoretical work (Syono and Yamasaki 1966, Charney 1973, Bates 1973, Koss 1976, Mak 1981, Fraedrich and McBride 1989, Winn-Nielsen 1993). CISK attributes TC growth to the interaction between individual cumulonimbi and large-scale moisture convergence. The release of latent heat in the troposphere leads to a lowering of the surface pressure which in turn increases the secondary surface circulation. The secondary circulation initiates when Ekman-induced frictional convergence induces an in-up-out circulation. In this scenario, as low level vorticity and Ekman pumping increase so does the latent heat release, which in turn lowers the pressure. This has the effect of causing a positive feedback loop. Observational studies by Leary and Thompson (1976) and Davidson et al. (1990) both view the genesis process as being driven by low-level convergence and a CISK-like mechanism. Leary and Thompson (1976) show the warm core in a depression, using rawinsonde data, to be about $2^{\circ} \mathrm{C}$ at $1.5 \mathrm{~km}, 3-4^{\circ} \mathrm{C}$ at $3.0 \mathrm{~km}$, and less then $3^{\circ} \mathrm{C}$ at $250 \mathrm{hPa}$. However, they do not show evidence of why this warming occurred except to say that there was a warming of the entire troposphere due to a CISKlike mechanism. 
More recent theories of tropical cyclogenesis have framed the debate as either being top-down or bottom-up. Both of these approaches assume that the inner core of the cyclone is thought to evolve from a Mesoscale Convective System (MCS). Some MCS's develop a mesoscale convective vortex (MCV) in the warm-core stratiform region which trails the leading edge of convection (Gamache and Houze 1982, Leary and Rappaport 1987, Brandes 1990, Chen and Frank 1993). Bosart and Sanders (1981), Velasco and Fritsch (1987), Menard and Fritsch (1989), Frank and Chen (1991), Chen and Frank (1993), Simpson et al. (1997), and Rodgers and Fritsch (2001) have all hypothesized that if a MCS moves into an area favorable for genesis to occur, the upper level warm core in the stratiform rain region might play a crucial role in tropical cyclogenesis. Gamache and Houze (1982) established that MCV's can form in the stratiform cloud deck of MCSs over tropical oceans. The main debate between the top-down and bottom-up theories is over the role of the MCV in genesis and in the initial formation of the warm core.

Top-down theories propose that the MCS pre-conditions the environment so that new convection does not initiate cold downdrafts that bring low $\theta_{\mathrm{e}}$ air into the BL. After the mid-levels increase their humidity, the MCV is advected to the surface and acts as the initial vortex that starts the genesis process. Simpson et al. (1997) emphasizes the importance of MCV mergers during the formation of TC Oliver during TOGA COARE. The mergers increase the vertical extent of the resulting vortex so a surface connection can be established. The vorticity rich MCV environment reduces the Rossby Radius of Deformation, which allows for the more efficient warming of the troposphere. Warming is found in the upper troposphere from the release of latent heat and in the midtroposphere from subsidence warming. Harr et al. (1996) used Omega dropwindsondes 
(ODWs) to investigate the formation of typhoon Ofelia (1993). Ofelia formed from one persistent MCS. Although Ofelia's formation is viewed as a top-down, Harr et al. (1996) found warm, dry mid-level air near the circulation center and believes that subsidence induced on the concave side of the main convective band might have played a role in warming the troposphere and in the formation of an eye.

More recent theories of early stage development include the WISHE theory (Emanuel 1989, Bister and Emanuel, 1997). In the Tropical Experiment in Mexico (TEXMEX) studies Bister and Emanuel suggest that after an MCS has formed with a cold core in the lower atmosphere and a cyclonic circulation in the mid-troposphere, the troposphere becomes nearly saturated on the mesoscale in the vortex core. This allows for an increase in the mid-tropospheric moist static energy so that evaporation of rain does not generate downdrafts that bring cool dry air to the BL. Enhanced surface fluxes associated with increased surface winds near the core will increase the BL moist static energy. After convection occurs, the temperature of the vortex core will increase, and the WISHE mechanism can then act as a positive feedback to the warm core cyclone.

The acceptance of the WISHE theory for more mature TCs is widespread among the scientific community. Some have proposed that the intensification process should be split, with a pre-WISHE phase, in which some other mechanism spins up a vortex to a critical point at which the WISHE theory can then take over.

Bottom-up theories suggest that a low-level vorticity rich environment in concert with deep convection can initiate tropical cyclogenesis. One theory that has been hypothesized is the axi-symmetrization of vorticity centers (Montgomery and Kallenbach 1997, Molinari et al. 2004, Reasor et al. 2005). In this theory, vorticity is increased in 
localized areas by vortical hot towers (cumulus clouds with high vorticity) embedded in a well organized but weak low-level cyclonic circulation. Axi-symmetrization and the stretching of these vorticity centers intensify the initial surface vortex to a critical point at which time WISHE takes over.

In an attempt to model the formation of TC Diana (1984), Hendricks et al. (2004) use a high resolution, $3 \mathrm{~km}$, near-cloud-resolving model. The small grid spacing allows for the examination of deep convection and its role in the development of Diana. The simulation shows the presence of vortical hot towers (VHTs). These are cores of deep convection that possess strong vertical vorticity. They develop from buoyancy-induced stretching of the local absolute vertical vorticity in an area of pre-existing low-level vorticity. At this grid spacing, VHT's are the preferred mode of convection. VHTs effectively trap the latent heat from the moist convection because of their high vorticity. These diabatically generated low-level PV anomalies are shown to axi-symmetrize and merge, an important step in the intensification of the newly formed vortex (Montgomery and Lu 1997, Montgomery and Enagonio 1998, Montgomery and Franklin 1998, Enagonio and Montgomery 2001, Hendricks et al. 2004, Montgomery et al. 2006). Peak warming of $5^{\circ} \mathrm{K}$ occurs at mid-levels in the core region due to localized subsidence. The broad regional warming of $1^{\circ} \mathrm{K}$ is primarily due to the imbalance of diabatic heating and adiabatic cooling in the convective region.

Stossmeister and Barnes (1992) documented the development of a secondary vortex in Tropical Storm Isabel. This new vortex developed into a new circulation center. The new circulation center developed just outside of the RMW and formed beneath the downwind anvil of intense convection in a rainband. They hypothesized that 
subsidence warming beneath the anvil, similar to the meso-lows found at the trailing edge of MCSs, could lower the pressure and serve as the initial perturbation for a TC. Heymsfield et al. (2006) investigated the structure of Tropical Storm Chantal in a highly sheared environment. Although Chantal failed to intensify into a hurricane, detailed analysis of the storm provides clues to warm core formation. The low-level circulation center is observed $80 \mathrm{~km}$ west-southwest of the deep convection in a rain free region. Two broad regions of warming are found. One area of warming is believed to be due to subsidence from the interaction of the high wind shear (Ritchie and Elsberry, 2001). This area had a maximum temperature perturbation at $500 \mathrm{hPa}$ and was located partially over the low level circulation center. Another area of warming was found to have a maximum at the $700 \mathrm{hPa}$ level. Heymsfield et al. (2006) hypothesized that this area of warming was due to similar mechanisms as found by Stossmeister and Barnes (1992).

Tropical Storm Humberto offers a new and competing view of genesis, where the warm core is found in the lower troposphere unlike what would be expected from many of the theories of genesis discussed in the previous sections. The nascent warm core at low-levels seems to be an important ingredient in the beginning stages of a TC. It causes the initial drop in pressure at the surface and acts to cap the BL. As air flows into the nascent eye, the energy content increases until the energy is released from the cap on the down shear side of the warm core and forms the nascent eyewall. As this occurs the deep convection located $50 \mathrm{~km}$ to the north of the circulation center moves towards the nascent eyewall and merges with it. This series of events show one possible path in which an MCS can evolve into a warm-cored structure and intensify into a hurricane. 


\subsection{Goals}

The Humberto dataset provides the opportunity to pose a series of questions about how the inner $200 \mathrm{~km}$ of the vortex causes or responds to intensity change. I investigate the following questions as TC Humberto deepens from a $1000 \mathrm{hPa}$ tropical depression to a $984 \mathrm{hPa}$ category 2 hurricane, and then weakens to $992 \mathrm{hPa}$ category 1 hurricane:

(1) How does the warm core evolve over the three days? Is the warm core in the upper troposphere as we find in mature TCs or in the lower troposphere? Where does the warm core form with respect to convective elements and do the observations serve to verify the WISHE theory whereby the warm core should appear strongest in the upper troposphere? This will be the first observational study to be able to depict the evolution of the warm core.

(2) What is the spatial evolution of $\theta_{\mathrm{e}}$ over the three days? Is the region where the inflow is collecting substantial energy from the sea located near the circulation center or does the inflow acquire energy at much greater distances from the center? Do the $\theta_{\mathrm{e}}$ fields of Humberto evolve from an asymmetric to an axisymmetric pattern as the TC intensifies?

(3) How do the reflectivity fields (a proxy for the location of latent heat release and therefore a heat source) evolve over the three days? In particular do changes in the spatial structure of the convection lead to changes in intensity? 
(4) How is the environment (wind shear, SST, relative humidity) in which the TC is embedded changing? How are these environmental variations influencing the structure of the warm core?

(5) What is the structure of a highly sheared storm evolving at higher latitudes over sharp SST gradients? How do the structures in Humberto compare to the structures found in more intense low latitude TCs that are intensifying in a low shear, more homogeneous thermodynamic environment? 


\section{Chapter 2}

\section{DATA}

\subsection{Flight Plan and Aircraft Involved in the Experiment}

On September $22^{\text {nd }}, 2001$, one NOAA WP-3D flew at $600 \mathrm{hPa}$, the NASA DC-8 flew at $300 \mathrm{hPa}$, and the Lockheed ER-2 flew at the $50 \mathrm{hPa}$ level. The flight path for the NOAA WP-3D and the NASA DC-8 are displayed in Figs. 9a and $\mathrm{b}$ respectively. The ER-2 flew a figure 4 pattern dropping GPS sondes 2 degrees from the low-level circulation center. On September $23^{\text {rd }}$ and $24^{\text {th }}$, NOAA added an extra WP-3D (flight level of approximately $800 \mathrm{hPa}$ ) and the Gulfstream-IV which sampled the TC's environment. The two WP-3Ds sampled the inner core out to about two degrees from the storm center making three passes each through the circulation center. The DC-8 flew a similar pattern to the WP-3D's except with a slightly wider sampling area. The ER-2 sampled the storm out to about four degrees from the center of the circulation. Figure 10 displays the approximate flight patterns for the $23^{\text {rd }}$ and the $24^{\text {th }}$ of September.

\subsection{GPS Dropwindsonde Data}

The National Center for Atmospheric Research (NCAR), in a combined effort with NOAA and the German Aerospace Research Establishment, developed a dropwindsonde based on the Global Positioning System (GPS) satellite navigation. The new GPS sonde was first operational in 1997 and provided reliable wind and thermodynamic profiles in TC Guillermo (Aberson et al. 2006).

\subsubsection{Number of Dropwindsondes Deployed and their Distribution}

On the $22^{\text {nd }}$ of September a total of 30 GPS sondes were deployed. About $95 \%$ of these recorded data successfully. The WP-3D dropped 15 GPS sondes. The ER-2 
dropped 2 GPS sondes, sampling from the stratosphere to the surface, while the DC-8 jettisoned 13 GPS sondes. Figure 11 displays the location of the DC-8 and ER-2 sondes. Figure 12 shows the location of the WP-3D sondes. In Fig. 12 when two four digit numbers are superimposed on each other it is usually an indication that one of the drops failed and another was released immediately.

On the $23^{\text {rd }}$ of September 96 GPS sondes were deployed. The WP-3D at $1.5 \mathrm{~km}$ altitude jettisoned 24, the WP-3D at $4 \mathrm{~km}$ released 45, the DC-8 at $11 \mathrm{~km}$ dropped 24, and the ER-2 released 3 sondes. Figure 13 displays the location of the DC-8 and ER-2 GPS sondes. Figures 14 and 15 show the locations of the GPS sondes jettisoned by the WP-3Ds. Similar to the previous figures, when two four digit numbers are superimposed on each other it is usually an indication that one of the drops failed and another was released immediately.

On September $24^{\text {th }} 102$ GPS sondes were released. The low-level WP-3D jettisoned 29, the WP-3D at $4 \mathrm{~km}$ released 48, the DC-8 dropped 21, and the ER-2 released 4 sondes. The location of the drops is not shown as it is similar to the pattern on the $23^{\text {rd }}$ of September.

\subsubsection{GPS Dropwindsonde Instrumentation}

The combined requirements of NOAA and NCAR led to certain design specifications for the GPS sonde. These include: global operation at altitudes up to 24 $\mathrm{km}$, deployment at indicated airspeeds up to $250 \mathrm{~m} \mathrm{~s}^{-1}$, simultaneous operation of up to four sondes per aircraft, radio frequency transmission bandwidth of $<20 \mathrm{kHz}$, telemetry range of $325 \mathrm{~km}$, GPS sonde descent time of $\sim 12$ min when released at $12 \mathrm{~km}$, sensor 
measurement rate of $2 \mathrm{~Hz}$, and a shelf life of at least 3 years. The GPS sonde and its various components are shown in Fig. 16.

The pressure, temperature, and humidity $(\mathrm{PTH})$ sensors are contained in a single module. The pressure sensor is a Barocap, a capacitive unit $3 \mathrm{~mm}$ across with a silicon diaphragm. The humidity sensor is the H-Humicap, a capacitive thin film dual-element sensor. The temperature sensor is the Vaisala Thermocap and is taken from the RS-80 radiosonde. This sensor is a more rugged sensor than previous thermocaps but produces a detectable response lag in the sondes temperature measurements that must be corrected for in the post-processing of the data. Winds are measured through use of the GPS sensor. The GPS receiver in the dropwindsonde tracks the relative Doppler frequencies from the radio frequency (RF) carrier of the GPS satellite signals, digitalizes them, and sends them back to the aircraft. The aircrafts data system GPS processing equipment then converts the Doppler frequencies into winds.

The GPS sondes sample data at $2 \mathrm{~Hz}$ so they provide high vertical resolution of about 6-7 meters in the lower atmosphere. Wind errors from the sondes are on the order of $0.5 \mathrm{~m} \mathrm{~s}^{-1}$ while temperature and pressure errors are on the order of $0.2^{0} \mathrm{C}$ and $1.0 \mathrm{hPa}$, respectively. Relative humidity errors average less than 5\%; however sensor wetting can still be a problem. Bogner et al. (2000) and Barnes (2008) offer schemes to correct this problem. Table 1 displays the operating range, accuracy, resolution, and time constants for the GPS sonde (Hock and Franklin 1999).

\subsection{Aircraft Data}

All the aircraft data used here are from the WP-3D. Both WP-3Ds measure flight level temperature, moisture, pressure, D-value, and winds. Details of the aircraft 
instrumentation can be found in Jorgensen (1984a) and Aberson et al. (2006). Both WP3Ds also deploy airborne expendable bathythermographs (AXBT's). AXBT's measure ocean temperatures from the surface to a depth of 300 meters. Since their first meteorological flights, electronic equipment and expendable probes have become more accurate, lighter, faster, and smaller. The aircraft data are output at $1 \mathrm{~Hz}$. The instruments, their range, resolution, and accuracy for the WP-3Ds are displayed in Table 2.

\subsection{Aircraft Radar}

Two research radars are on the WP-3Ds. The lower fuselage (LF) radar operates at a wavelength of $5.5 \mathrm{~cm}$ (C-band). This radar scans horizontally at all azimuth angles and provides plan views of the TC. There can be errors in these data and one of the major problems is the large vertical beam width (4.1 degrees). This causes a decrease in vertical resolution at distances larger than 60-70 km (Marks 1985), resulting in inadequate beam filling. Errors due to attenuation of the radar beam are also a problem, although for this study the WP-3Ds make multiple passes through the storm center and outer bands. This allows for a comparison of radar images to get the most accurate reading of actual beam returns. Distant targets are often obscured but they become clear when the radar has an unobstructed view and is within the range parameters discussed above. The pitch and roll of the aircraft are also measured and allow the researcher to use only radar images that have not been contaminated by extreme variations of these two degrees of freedom. A pitch or roll of over 5 degrees can cause problems with the radar. Bright band errors are also prevalent when the radar is sampling areas of convection and stratiform rain where ice is covered with water (freezing level). Water covering the 
surface of ice returns a larger signal to the radar which can lead to a misinterpretation of the radar data. However, if recognized, bright bands offer important information on storm structure.

The tail radar (X-band, $3.2 \mathrm{~cm}$ wavelength) scans from 25 degrees ahead to 25 degrees behind the aircraft and rotates in a vertical plane. This radar also can suffer from extreme pitch and roll. The smaller radar wavelength also leads to larger problems with attenuation than the LF radar (Jorgensen 1984a).

Hundreds of LF and tail radar images were processed at the Hurricane Research Division (HRD). The continuous radar images allow for an almost continuous view of storm evolution throughout each of the three days of sampling. Table 3 (Jorgensen 1984a) shows the parameters for the lower fuselage and tail radar. 


\section{CHAPTER 3}

\section{Methods}

\subsection{Processing of GPS Dropwindsonde Data in ASPEN}

The data are initially processed using the Atmospheric Sounding Processing Environment (ASPEN) program. ASPEN, developed by NCAR, takes the raw data from the GPS sonde in the form of Airborne Vertical Atmospheric Profiling System (AVAPS) files. ASPEN uses an extensive series of quality control algorithms to systematically identify spurious data and either corrects or removes these from the data set.

Temperature, humidity, and pressure are all processed separately by ASPEN. The winds, which are calculated by the relative Doppler frequencies from the radio frequency (RF) carrier of the GPS satellite signals, are given as $u$ and $v$ components. Whether or not wind data can be computed depends on the number of satellites which the GPS sonde connects with during its deployment. The Vaisala Thermocap (temperature sensor) used in the GPS sondes has a known problem with processing quick temperature changes. This temperature time lag problem is corrected for in ASPEN through the use of a simple equation:

$$
T=T_{m}+\left(\frac{\partial T}{\partial z}\right)\left(\frac{\partial z}{\partial t}\right) \times \tau
$$

where $\mathrm{T}_{m}$ is the measured temperature from the GPS sonde, $\partial \mathrm{T} / \partial \mathrm{z}$ is the measured lapse rate, $\partial z / \partial t$ is the fall velocity of the sonde and $\tau$ is a time constant that changes depending on temperature. Further information on the wind and temperature corrections can be 
found in Hock and Franklin (1999). After making the necessary corrections, ASPEN displays the raw and the quality controlled (QC) data and the QC data are displayed on a skew-T log-P diagram. A complete description of the QC procedures and details regarding the ASPEN algorithms can be found in the user manual: (http://www.eol.ucar/rtf/facilities/software/aspen/Aspen\%20Manual.pdf).

\subsubsection{Post Processing of GPS Dropwindsonde Data}

After processing the data with ASPEN, an investigation of all the GPS sondes is undertaken. All 228 skew-Ts are viewed for possible errors. Three main types of errors can be found after the data are processed with ASPEN. These include: sensor wetting, radio frequency interference (RFI), and humidity errors. Sensor wetting occurs when the GPS sonde falls through rain or cloud and the humidity sensor remains saturated. This is most obvious when the lapse rate becomes dry adiabatic in the mixed layer and the air remains saturated. This is corrected for by finding where the lapse rate turns dry adiabatic and then assuming a constant mixing ratio to the surface (Bogner et al. 2000). New relative humidity values are then calculated. A correction of this type is shown in Fig. 17. On rare occasions, the humidity sensor is not calibrated correctly, resulting in an underestimate of the relative humidity. If the temperature and the dew point are close and track each other, it is often the case that the humidity sensor is reading values that are slightly low. However, if the relative humidity reached $100 \%$ anywhere in the sounding, the sounding is considered to be reading the correct dew point. For cases where this did not occur the dew point is considered to be biased too low and the layer is then saturated. This case was very rare in the Humberto data. Wang (2005) found that there was no systematic dry bias in the GPS sonde data. The other error that can occur is RFI. This 
occurs when the radio signal is somehow interrupted as it is being transmitted to the aircraft. The skew-T's for these sondes often show very erratic behavior. This is corrected for by erasing the spurious data from the sounding. These errors are very obvious and an example is shown in Fig. 18.

\subsubsection{Processing GPS Dropwindsonde Data}

During the approximately 4 hours of GPS sonde deployment that took place on each of the three days, Humberto's mean sea level pressure fluctuated by only a few hPa. I assume steady state and composite the GPS sondes with respect to the circulation center for each day. A number of steps are involved in making the storm composites. These steps will be listed in the following section and apply to each day of the study period.

The first step is to determine the storm track. The accuracy of the storm track is crucial because the tangential and radial components are a function of the circulation center location. Errors in the location of the circulation center can lead to large errors in the wind components especially for drops near the circulation center. Using the center fixes from the WP-3Ds as they fly the research missions gives the best estimate of the circulation center. However, the WP-3Ds drop GPS sondes as they ferry towards the TC and when they depart for base. Drops jettisoned before or after the center fixes will fall out of the range of the storm track data. Therefore, the best track data are used to supplement the WP-3D circulation center fixes. Time is plotted as the independent variable against both longitude and latitude. I then use a best fit for each of the 6 graphs (time versus longitude, time versus latitude, for 3 days). A least squares fit that is a cubic or a higher order polynomial induces unusual accelerations in the motion of the storm. 
Overall, three of the regression lines were linear fits and three were quadratic fits. All of the fits had $\mathrm{R}^{2}$ values of greater than 0.98 .

The GPS sonde records the time, pressure, temperature, relative humidity, the earth relative speed of the sonde, the direction of the sonde, latitude, longitude, and altitude. After post processing of all GPS sondes, the median drop time for each mission is chosen as the time around which all the data will be composited. The time at which each GPS sonde is jettisoned is input into the program to find its relative time to the composite time for the track of the storm on that day. The GPS sonde records the time at a $2 \mathrm{~Hz}$ frequency and this time is input into the equations for longitude and latitude to find its relative position to the moving storm center as the sonde falls to the sea surface. A correction for longitudinal distances is made depending on the latitude of the storm at each time recorded by the GPS sonde. Each variable output by the GPS sonde is therefore associated with a relative position to the moving storm center at each half second reading. This is done for every GPS sonde on each of the 3 days.

\subsubsection{Calculating Storm Relative Winds and Thermodynamic Variables}

A calculation of the storm relative $\mathrm{u}$ and $\mathrm{v}$ components is made for each time that the sonde has recorded data. The storms $\mathrm{u}$ and $\mathrm{v}$ winds are subtracted from the GPS $\mathrm{u}$ and $\mathrm{v}$ components to obtain storm relative winds. The radial and tangential winds may be calculated using:

$$
\begin{aligned}
& V_{r a d}=U_{r e l} \cos \Theta+V_{r e l} \sin \Theta \\
& V_{\text {tan }}=-U_{r e l} \sin \Theta+V_{r e l} \cos \Theta
\end{aligned}
$$


where $\mathrm{U}_{\text {rel }}$ is the $\mathrm{u}$ component of the storm velocity subtracted from the $\mathrm{u}$ component of the GPS sonde and $V_{\text {rel }}$ is the $\mathrm{v}$ component of the storm velocity subtracted from the $\mathrm{v}$ component of the GPS sonde. $\Theta$ is the angle between the GPS sonde and a Cartesian coordinate system aligned along a unit circle. Vapor pressure, saturation vapor pressure, mixing ratio, specific humidity, potential temperature, the temperature at the lifted condensation level (LCL), and $\theta_{\mathrm{e}}$ are calculated following Bolton (1980).

\subsubsection{Missing Data and Interpolation Method}

Occasionally the soundings obtained from the GPS sondes have data gaps. These gaps can be due to transmission problems, instrument failure, or RFI. If the gaps are 300 $\mathrm{m}$ thick or less I employ a linear interpolation to assemble a more complete sounding. Gaps larger than $300 \mathrm{~m}$ are not used in the composites. Even after using this process, the data are sampled at differing heights for each GPS sonde. To overcome this problem a method is used that places the data into constant height intervals. This involves taking data that are sampled every 6-7 meters in the lower troposphere (larger sampling distances in upper troposphere) and using a linear interpolation to adjust it to height intervals of 10 meters. Using this process allows for high vertical resolution and also transfers all the data into a format that is easy to process.

\subsection{Testing Different Methods for Making Plan View Maps}

The data from each GPS sonde are formatted into $10 \mathrm{~m}$ height intervals and composited in space and time with respect to storm motion, then plan view maps are created. I tested three methods for making the plan view maps. The Barnes Objective Analysis is applied first because of its extensive use in meteorology (Barnes 1964). This method is used to fit unevenly spaced data into an evenly spaced matrix. The Barnes 
scheme uses a Gaussian weighting function in which the weight that each station contributes to the overall grid values drops off rapidly with increasing distance from the station. Since the tails of a Gaussian function are infinite, a radius of influence is chosen so stations outside this influence are not affected. For each grid point, observations in the radius of influence are assigned a weight value $\mathrm{W}_{1}$ using the formula:

$$
W_{1}=\exp ^{-\left(\frac{d}{R}\right)^{2}}
$$

where $\mathrm{d}$ is the distance from the observation to the grid point and $\mathrm{R}$ is the radius of influence. $\mathrm{R}$ is determined by the average distance of each datum to all other data points. It can then be manipulated by multiplying it by a constant. There are two determining factors that must be met for R. First, at least 3 observations are required to determine an interpolated value at a grid point. Therefore, the search radius should be chosen large enough so that this requirement is met. This depends on the number of data reports present, as well as their distribution. Second, the search radius should not be too large. If it is too large, many observations will affect each grid point, resulting in an analysis that is overly smoothed. After the weights are determined, the first guess of the grid point is determined by: 


$$
X_{g}=\left(\frac{\sum W_{i} X_{i}}{\sum W_{i}}\right)
$$

This is known as the first pass. $\mathrm{W}_{\mathrm{i}}$ is the weight function in equation (5) at each grid point. $X_{\mathrm{i}}$ is the value of each successive observation and $\mathrm{X}_{\mathrm{g}}$ is the value assigned to each successive grid point. Typically 2 passes are performed. A method known as successive correction is used. This method interpolates from the new grid values back to the original station. The difference from the original value is used as a correction to the first pass grid value. A new parameter known as the convergence parameter (gamma or $\Gamma)$ is used to control the amount of smoothing. Each correction step may be represented as:

$$
X_{i}^{1}=X_{g}+\left(\frac{\sum W_{i}^{1}\left(X_{i}-X_{a}\right)}{\sum W_{i}^{1}}\right)
$$

where $\mathrm{X}_{\mathrm{a}}$ is the first pass grid value and $\mathrm{W}^{1}$ is the correction weight parameter:

$$
W_{i}^{1}=\exp ^{-\left(\frac{d}{R \Gamma}\right)^{2}}
$$

The convergence parameter gamma ranges between 0 and 1 . A value of .2 or .3 is generally used. The successive correction may be used more than once in an attempt to 
adjust the fields to take on values closer to the original observations. Figure 19 has the radial wind field plotted at 50 meters with the Barnes Objective analysis technique. Included on the plot are the actual values of the GPS sonde at that height.

An alternate method to construct plan views is to use a cubic interpolation scheme developed by Matlab. This method uses a piecewise cubic Hermite interpolation (Fritsch and Carlson 1980) to analyze the data. The cubic spline method tends to preserve the monotonicity and the shape of the data. Figure 20 shows the radial wind field plotted at 50 meters with the cubic spline method. Included on the plot are the actual values that the GPS sondes read at that height.

The data is also analyzed subjectively. The goal in these analyses is to capture the mesoscale aspects of the data without losing the high spatial resolution of the GPS sonde data and then to compare this method with the other analyses. Figure 21 displays the same radial wind analysis as Figs. 19 and 20, except the analysis is done subjectively.

The objective of testing these different methods is to find a way to capture the most realistic view of the storm's structure. Of course this is a composite analysis, so we are not getting a true "snapshot" of the storm. It is similar to taking a picture with the shutter open for a long period. The period that the aircraft are flying Humberto on each day is approximately three hours. We consider the subjective analysis to be "truest", because of the analyst's meso-beta knowledge of a TC. Comparisons of many fields were made with all three methods. These included: radial wind, tangential wind, temperature, specific humidity, and equivalent potential temperature. In each case the use of the piecewise cubic Hermite interpolation came closest to the subjective analysis, with most fields looking virtually identical. In Fig. 19, the Barnes objective analysis does 
not capture the high radial wind values close to the storm center. The Barnes objective analysis only captures $16 \mathrm{~m} \mathrm{~s}^{-1}$ compared to the 25 to $30 \mathrm{~m} \mathrm{~s}^{-1}$ inflow in the subjective and cubic spline analysis. It also gives top values of $4 \mathrm{~m} \mathrm{~s}^{-1}$ for the outflow plan view compared to the $15 \mathrm{~m} \mathrm{~s}^{-1}$ outflow from the subjective and cubic spline analysis. The Barnes scheme is made to objectively interpolate unevenly spaced data to a regular shaped Cartesian grid. In most cases this is unevenly spaced data has fairly regular distances between each point, such as with the rawindsonde network in the U.S. The flight plans and drop patterns for Humberto and most TCs are specifically designed to capture the higher gradients in and around the eye and eyewall. Therefore, the highest spatial density of GPS sondes is in and around the eyewall. Moving further away from the storm center the data become sparser. Many attempts were made to adjust the parameters in the Barnes objective scheme to capture a more "realistic" view of the storm. $\mathrm{R}$ was made larger and smaller. Too large an $\mathrm{R}$ and the fields became too smoothed. Too small an $\mathrm{R}$ and parts of the grid would not register any values. Gamma was also adjusted. The largest gamma of 1 gives a Gaussian distribution with an infinite tail and the fields become overly smoothed. Smaller gammas of .1 and .2 gave results that look similar to Fig. 19. Multiple passes are made; so that the successive corrections can bring the grid point values closer to the actual data. This yielded moderate improvements, however the densely packed data near the eye and eyewall are still not resolved. A maximum of 5 passes were tried.

The cubic spline is able to capture both the large scale features and the dense data in the inner core with considerable fidelity (Fig. 20). The cubic spline does have some problems however. Comparing Fig. 20 with Fig.21, the field made with the cubic spline 
is not as smooth and does contain some sharper angles that would not be present in a TC. It does however match up with the actual values shown on the plan view. One of the only variables that is not well represented by the cubic spline interpolation is the specific humidity. This is because the specific humidity may vary highly in space and time. If two points are very close together and the values are markedly different, the cubic spline will over shoot and create values nearby that are too high. This is not a problem with the wind, temperature, or equivalent potential temperature fields, because these fields tend to not be as variable. Luckily there was a plethora of data for all three days. In cases tested with only a few points, the cubic spline sometimes produces extremely sharp angles and very large gradients, especially at the edges of the grid. However, plan views with few data points are not used in these analyses.

\subsection{Creating Three-Dimensional Matrices}

Using the cubic spline to construct plan view maps, I then construct threedimensional matrices of each field. The three-dimensional matrix is created by simply stacking the plan view maps at every 10 meters and keeping the grid boundaries the same. Once a three-dimensional matrix is constructed, I may slice through it at any point and in any direction to get a vertical cross section. For some of the meteorological variables this was possible through a large depth of the troposphere, due to the fact that the DC-8 and the ER-2 were dropping GPS sondes at high altitudes. The highest resolution is in the lower troposphere where GPS sondes from all four aircraft are found.

\subsubsection{Temperature Perturbation Vertical Cross-Sections}

The construction of the temperature perturbation vertical cross-sections is carried out first by making a three-dimensional matrix of the temperature fields. Using the 
latitude and longitude of the center of the storm composite, vertical slices can be assembled at any angle through this center point. At each 10 meter interval in the vertical cross-sections, the arithmetic average at each height is computed. The anomaly of this mean value is calculated. This is done for every height from the surface to $10 \mathrm{~km}$ out to a radial distance of one and a half degrees from the circulation center. This method was used for the second and third days of sampling when Humberto was above hurricane strength. Ten $\mathrm{km}$ is the height at which the DC-8 was dropping GPS sondes, so there is still reasonable resolution at this altitude. Since the warm core spreads out with height in a mature TC (Hawkins and Rubsam 1968), the lower GPS sonde density in the upper troposphere is not a major problem. When Humberto is a tropical storm, the three dimensional temperature matrix is calculated up to a $5 \mathrm{~km}$ altitude and a radial distance of about one degree from the circulation center. This is necessary because not as many sondes were jettisoned from the upper troposphere.

\subsubsection{Calculation of Convective Available Potential Energy}

Convective available potential energy (CAPE) (Moncrieff and Green 1972) is one of the most well known indices to measure conditional stability. It is not often that measurements of CAPE are made in TCs. A common way to measure CAPE is to use the virtual temperature. The moisture sensor has a greater failure rate than the temperature sensor, which can leave large data gaps in the constructed fields. I use only temperature in the CAPE calculations, therefore a bias is introduced 10\% underestimate (Doswell and Rasmussen 1994). Therefore the formula for calculating CAPE is: 


$$
C A P E=g \int_{L F C}^{E L} \frac{T-T e}{T e} d z
$$

where $\mathrm{g}$ is the gravitational acceleration, $\mathrm{T}$ is the temperature of the parcel, and $\mathrm{Te}$ is the environmental temperature. The integration is performed from the level of free convection (LFC) to the equilibrium level (EL). This is equivalent to the positive area between the environmental temperature profile and the undiluted ascent of a parcel lifted from the BL seen on a thermodynamic diagram. To represent the BL parcel an average of the lowest 500 meters is used for the mixing ratio and the potential temperature. Theoretically an ascending parcel in a mature hurricane near the vortex center should follow lines of constant angular momentum, which angle radially outward with height in a TC. However, I calculate CAPE by lifting the parcel vertically up each column in the three-dimensional matrix. The integration is accomplished in increments of 10 meters. Since the warm core is concentrated near the TC center, this can lead to an underestimate of the actual CAPE.

The three-dimensional temperature matrix has the best data coverage up to a height of about 5000 meters on the $22^{\text {nd }}$ and up to 10,000 meters on the $23^{\text {rd }}$ and $24^{\text {th }}$. A detailed examination of the GPS sondes jettisoned from the stratosphere and upper troposphere reveals that the lapse rate is moist adiabatic above $5 \mathrm{~km}$ in the analysis area. I extend the temperature profiles from the top of the matrix to the bottom of the tropopause using the moist adiabatic lapse rate equation (Stull 2000): 


$$
\frac{\partial T}{\partial Z}=\frac{\frac{g}{C_{p}}\left(1+\frac{r_{s} \times L_{v}}{R_{d} \times T}\right)}{\left(1+\frac{L_{v}^{2} \times r_{s} \times \varepsilon}{C_{p} \times R_{d} \times T^{2}}\right)}
$$

where $\frac{\partial T}{\partial Z}$ is the saturated adiabatic lapse rate, $r_{s}$ is the saturation mixing ratio, $L_{v}$ is the latent heat of vaporization, $R_{d}$ is the gas constant for dry air, $T$ is the temperature, $\varepsilon$ is the ratio of the gas constants for dry air and water vapor, and $C_{p}$ is the specific heat of air at constant pressure. Since the mixing ratio is calculated from both the pressure and the vapor pressure, the pressure has to be calculated at every level hydrostatically.

The moist adiabatic lapse rate is solved recursively, stepping upward from an initial temperature. Each step must take a small enough height increment that the moist lapse rate does not change significantly. The height increment used is 10 meters. As a second check on this calculation, $\theta_{\mathrm{e}}$ is calculated for these moist adiabats. Equivalent potential temperature remained constant in these calculations, confirming that the lapse rate is pseudoadiabatic.

The bottom of the tropopause, where the temperature starts to increase with height, is found by looking at the ER-2 GPS sondes. This point is near $13.5 \mathrm{~km}$ and is the height used for calculating CAPE. This will also lead to a slight underestimate of the actual CAPE, because the parcel would ascend along its moist adiabat until it intersected the environmental temperature profile. 


\subsubsection{Calculation of Convective Inhibition}

For an air parcel containing CAPE, Convective inhibition (CIN) represents the negative area on a thermodynamic diagram. If CIN is sufficiently large, then deep convection will not form. The formula for calculating CIN is:

$$
C I N=\int_{P H}^{L F C} g \frac{T-T e}{T e} d z
$$

where $\mathrm{g}$ is the gravitational acceleration, $\mathrm{T}$ is the temperature of the parcel, and $\mathrm{Te}$ is the environmental temperature. The integration is performed from the mixed layer parcel height $(\mathrm{PH})$ to the LFC. This represents the negative area due to undilute ascent by a parcel lifted from the BL. To represent the parcel mixing ratio and potential temperature an average of the lowest 500 meters is used.

\subsection{Plan View Using the AXBT's}

A total of 64 AXBT's were deployed from the WP-3Ds over the three days of study. On the $22^{\text {nd }}, 12$ were dropped. On the $23^{\text {rd }}, 24$ AXBT's were dropped and on the $24^{\text {th }}, 28$ AXBT's were jettisoned. The AXBTs are used only for sea surface temperatures. The same piecewise cubic Hermite interpolation that is used for the GPS sonde is used to create plan views of the SSTs that the tropical system is encountering.

\subsection{Calculation of Variables from Aircraft Data}

Observations from the WP-3D's are at $1 \mathrm{~Hz}$ and are treated in a manner similar to the GPS sonde data. The temperature and dew point needed corrections for times when the WP-3D is in cloud or heavy rain. If the dew point temperature exceeds the 
temperature, I calculate the average, so that the temperature equals the dew point, similar to the method used by Zipser et al. (1981). Measurements of $\theta_{\mathrm{e}}$, tangential wind, and vertical velocity in the eye and eyewall will help elucidate the relationship between the thermodynamics in the BL estimated from the GPS sondes and measurements from the aircraft in the eyewall

\subsection{Calculation of the Vertical Wind Shear}

The vertical shear of the horizontal wind for the synoptic scale environment of Humberto is calculated using the SHIPS model (DeMaria and Kaplan 1999, DeMaria et al. 2005). The SHIPS model is a statistical-dynamical model which runs using input from the Global Forecast System (GFS) model. The model is developed using multiple regression techniques with numerical model forecasts, climatology, and persistence as predictors. The vertical shear of the horizontal wind is evaluated for the $850-200 \mathrm{hPa}$ layer. The vertical shear calculation uses an annulus around the storm from 200 to 800 $\mathrm{km}$ out from the storm center. The shear is calculated from the two annuli at 850 and 200 $\mathrm{hPa}$. Averaging around the annuli removes the storm circulation.

\subsection{Energy Budget of Inflow}

A subjective analysis of the GPS sondes is performed to create plan views of all the variables for the energy budget. The subjective analysis is used for this part of the study because the cubic spline does not perform well on the edges of the fields when the data are sparse, as it is in some areas on the $22^{\text {nd }}$. Temperature, specific humidity, and ground relative total wind speed at $10 \mathrm{~m}$ are used to estimate the surface fluxes via the bulk aerodynamic equations. The inflow path is determined from the inflow layer mean. Three soundings of $\theta_{\mathrm{e}}$ are chosen along the inflow path and represent the increase in 
energy of the inflow moving towards the nascent circulation center. These soundings of $\theta_{\mathrm{e}}$ are created from the plan views of $\theta_{\mathrm{e}}$.

Equivalent potential temperature is used in the budget scheme because it is conserved in the presence of the evaporation of rain or spray and it is has been related directly to TC intensity (Malkus and Riehl 1960, Emanuel 1986, Betts and Simpson 1987). An energy budget equation for $\theta_{\mathrm{e}}$ from Wroe and Barnes (2003) is used and can be written as:

$$
\frac{\partial \overline{\theta_{e}}}{\partial t}+\overline{u_{j}} \frac{\partial \overline{\theta_{e}}}{\partial \overline{x_{j}}}-Q_{r}+\frac{\partial\left(\overline{\left.w^{\prime} \theta_{e}^{\prime}\right)}\right.}{\partial z}-\varepsilon=0
$$

where $u_{\mathrm{j}}$ denotes the mean or grid-scale components of the three-dimensional velocity vector, $x_{\mathrm{j}}$ are the spatial components of a three-dimensional vector, $t$ is time, $Q_{\mathrm{r}}$ is the radiative divergence, and $\varepsilon$ equals the viscous dissipation. Henceforth, the mean is represented as the variable $\left(\theta_{\mathrm{e}}=\right.$ mean quantity $)$ and the prime equates to the subgridscale component. Equation (11) states that the local rate of change of $\theta_{\mathrm{e}}\left(\partial \theta_{\mathrm{e}} / \partial t\right)$ in the inflow column plus the grid-scale advection $\left(u_{\mathrm{j}} \partial \theta_{\mathrm{e}} / \partial x_{\mathrm{j}}\right)$, minus the radiative divergence $\left(Q_{\mathrm{r}}\right)$, plus the subgrid surface fluxes in the vertical, minus viscous dissipation $(\varepsilon)$ equal zero. I have made the established assumption that the subgrid-scale fluxes in the horizontal are substantially smaller than the subgrid-scale vertical fluxes and can be neglected (Wroe and Barnes 2003). In addition, dissipation is neglected, which is valid due to the relatively low wind speeds found when Humberto is a tropical storm (Bister and Emanuel 1998, Zhang and Altshuler 1999).

The mean cooling rate from radiation in a clear tropical environment is estimated to be $1-2 \mathrm{~K} \mathrm{day}^{-1}$ (Albrecht and Cox, 1975). This equates to a cooling rate of 
approximately $0.125-0.25 \mathrm{~K}$ over the 3 hours in which GPS sondes were jettisoned on the $22^{\text {nd }}$. The inflow path is under stratiform clouds so the cooling rate will be less than this estimate and may be neglected. Invoking a constant flux layer, the turbulent fluxes rapidly dominate the molecular transfer term that controls the fluxes at the air-sea interface. Therefore I use the bulk aerodynamic equations to approximate the surface sensible and latent heat fluxes.

Under the steady-state assumption used to make the composite, I assume that a streamline represents a trajectory. The steady-state assumption by definition neglects the local rate of change $\left(\partial \theta_{\mathrm{e}} / \partial t\right)$. This assumption is supported by the fact that Humberto's mean sea level pressure (MSLP) changes by only a few hPa over the 3 hours of the experiment. Therefore the equation is:

$$
V \frac{\partial \theta_{e}}{\partial \eta}+w \frac{\partial \theta_{e}}{\partial z}=-\frac{\partial\left(\overline{\left.w^{\prime} \theta_{e}^{\prime}\right)}\right.}{\partial z}
$$

where $V=$ horizontal velocity along the inflow trajectory, $\eta=$ the distance along the trajectory, $w=$ vertical velocity, and $z=$ height. The first term on the lhs of (12) is the product of the velocity along the inflow trajectory and the gradient of $\theta_{\mathrm{e}}$ estimated along the trajectory. The second term on the lhs of (12) is the product of the vertical velocity and the vertical gradient of $\theta_{\mathrm{e}}$. If convergence is present along the inflow trajectory, it can compress and raise the height of the mixed inflow layer. This is not an increase in the energy content of the inflow but instead a redistribution of the energy. The first term on the rhs of equation (12) corresponds to the subgrid-scale fluxes at the air-sea interface and the transfer of energy through the top of the layer of air being examined. 


\subsubsection{Calculation of Divergence and Vertical Velocity}

Divergence is calculated along the inflow path. To obtain the best possible estimate of divergence, subjective analyses of the $\mathrm{u}$ and $\mathrm{v}$ wind components are constructed. The inflow trajectory is placed on the plan view maps of the $u$ and $v$ components. Eight equally spaced points are placed on the trajectory and the divergence is calculated by using the incompressibility assumption yielding the continuity equation as:

$$
\frac{\partial u}{\partial x}+\frac{\partial v}{\partial y}+\frac{\partial w}{\partial z}=0
$$

where $\mathrm{u}$ and $\mathrm{v}$ are the storm relative components of the wind, $\mathrm{x}$ and $\mathrm{y}$ are the distances along longitude and latitude, $\mathrm{w}$ is the vertical velocity and $\mathrm{z}$ is the height in meters (Holton 2004). The $\mathrm{x}$ and $\mathrm{y}$ increments are $25 \mathrm{~km}$ and divergence is calculated at all 8 points every $100 \mathrm{~m}$ from the surface to $1000 \mathrm{~m}$. To calculate the vertical velocity in the lower $1000 \mathrm{~m}$ along the inflow trajectory, equation (13) is integrated at $100 \mathrm{~m}$ height intervals to obtain the vertical velocity at each height along the 8 points along the trajectory. 


\section{CHAPTER 4}

\section{Results}

\subsection{Large-Scale Environment}

As Humberto is sampled as a tropical storm (TS) on the $22^{\text {nd }}$, SSTs are above $28^{\circ}$ $\mathrm{C}$, the vertical wind shear (VWS) is low, and environmental relative humidity is low. Save a low environmental relative humidity, Humberto is developing in ideal conditions for further intensification on the $22^{\text {nd }}$. On the $23^{\text {rd }}$ and $24^{\text {th }}$, Humberto encounters higher wind shear, lower SSTs, and continued low environmental relative humidity. The following sections discuss the environmental fields in which this high latitude TC forms, intensifies, and then weakens.

\subsubsection{Formation of Humberto}

Humberto had its origin in a trough that extended southwest from the circulation of Hurricane Gabrielle. On September 18, a west-ward moving tropical upper tropospheric trough (TUTT) enhanced the deep convection and a weak low formed in the area. The system became a tropical depression near 1200 UTC September 21, when deep convection developed near the center of a broad cyclonic circulation. At about 1800 UTC September 21 Humberto's pressure began to drop while under the east side of the TUTT (Fig. 22). This is an area favorable for intensification where the $200 \mathrm{hPa}$ height field (see appendix Fig. 1) displays divergence of $2 \times 10^{-5} \mathrm{~s}^{-1}$.

Early on September $22^{\text {nd }}$, Humberto was a tropical depression with a minimum central pressure of $1010 \mathrm{hPa}$. The storm was located in the Atlantic basin near $29^{\circ} \mathrm{N}$ and $66^{0} \mathrm{~W}$. The movement was to the north-northwest at approximately $4 \mathrm{~m} \mathrm{~s}^{-1}$. Humberto would continue moving to the north and eventually would recurve to the northeast (Fig. 
23). When the first GPS sondes were being jettisoned late on the $22^{\text {nd }}$ Humberto's minimum central pressure was $1000 \mathrm{hPa}$ and it had attained tropical storm strength. Twenty four hours later, late on the $23^{\text {rd }}$, it would intensify to a category 2 storm and then weaken to a category 1 storm on the $24^{\text {th }}$ (Fig. 24). The GPS sondes on the $22^{\text {nd }}$ were deployed from approximately 1830 UTC to 2200 UTC.

\subsubsection{Evolution of Environmental Height Fields}

At about the time the last GPS sondes were launched on the $22^{\text {nd }}$, the Bermuda High is well established over the Atlantic with a maximum surface pressure of $1030 \mathrm{hPa}$ at $45^{\circ} \mathrm{W}$ and $43^{\circ} \mathrm{N}$ (Fig. 25a). There is a weakness in the ridge over Tropical Storm Humberto (Fig. 25b). At the same time, the TUTT at $200 \mathrm{hPa}$ is just west of Humberto

(Fig. 26). From 00 UTC on the $23^{\text {rd }}$ to 00 UTC on the $25^{\text {th }}$, the low-level and upper-level large-scale fields show no evidence of any synoptic disturbances in the vicinity of Humberto as it recurves around the Bermuda High. No changes in intensity are expected from the large-scale pressure or height fields.

\subsubsection{Evolution of Environmental Relative Humidity}

Environmental values of relative humidity from the SHIPS model show that Humberto is developing and maintaining its intensity in a dry environment. Environmental relative humidity in the $850-700 \mathrm{hPa}$ layer for the 3 days of the experiment has values of $60-65 \%$. In the $700-500 \mathrm{hPa}$ layer environmental relative humidity stays constant at 45-50\% and in the 500-300 hPa layer lower values of approximately $40-50 \%$ are present throughout the experiment. The dry air in which Humberto develops, intensifies, and then weakens will be shown to have a large impact on the structure of the warm core and therefore the intensity of the TC. 


\subsubsection{Evolution of Sea Surface Temperatures}

Throughout the experiment the SSTs change considerably along the path of the storm. As Humberto transitions from a depression to a tropical storm on the $22^{\text {nd }}$, SSTs near the circulation center are approximately $28.3^{\circ} \mathrm{C}$ (Fig. 27). From the $22^{\text {nd }}$ to the $23^{\text {rd }}$, there is a large north to south gradient in SSTs and temperatures near the circulation center drop from $28.3^{\circ} \mathrm{C}$ to $26.3^{\circ} \mathrm{C}$. From the $23^{\text {rd }}$ to the $24^{\text {th }}$, SSTs drop slightly from $26.3^{\circ} \mathrm{C}$ near the circulation center to $25.8^{\circ} \mathrm{C}$ on the $24^{\text {th }}$.

\subsubsection{Evolution of the Wind Shear}

As Humberto transitions from a depression to a tropical storm on the $22^{\text {nd }}$, the vertical wind shear is 5 to $7 \mathrm{~m} \mathrm{~s}^{-1}$ (Fig. 28). From the $22^{\text {nd }}$ to the $23^{\text {rd }}$, the vertical shear increases to $11 \mathrm{~m} \mathrm{~s}^{-1}$. As the vertical wind shear increases echo tops should decrease and the warm core aloft should weaken. However, the TC continues to intensify and the minimum central pressure drops by $20 \mathrm{hPa}$ from $22^{\text {nd }}$ to the $23^{\text {rd }}$. On the $24^{\text {th }}$, Humberto weakens to a category 1 storm while under similar wind shear conditions to the $23^{\text {rd }}$.

\subsection{Tropical Storm Humberto: Clues to Formation}

The next few subsections will focus on the formation of the nascent warm core and the thermodynamics that lead to the formation of the eyewall. Within the vicinity of Tropical Storm Humberto SSTs range from about $28^{\circ} \mathrm{C}$ to $28.5^{\circ} \mathrm{C}$ and the vertical wind shear is $6 \mathrm{~m} \mathrm{~s}^{-1}$. These are within the parameters for genesis (Gray 1968, 1975, 1979). Plan views and vertical cross-sections from the GPS sondes along with radar and in-situ flight level data are all utilized to elucidate the relationship among the reflectivity structure, the formation of the nascent warm core, and the development of the nascent eyewall. 


\subsubsection{GPS Sonde Analysis and Reflectivity Structure}

In Fig. 29, the composite picture shows that the minimum central pressure is about $1000 \mathrm{hPa}$ at 20 meters. The streamline analysis that is superimposed on the pressure field reveals the low-level circulation center is slightly west of the center of lowest pressure. The streamlines also reveal an area of confluence about $50 \mathrm{~km}$ to the north-northeast of the circulation center. The reflectivity field in Fig. 30 shows that an arc of deep convection, oriented north to east of the low pressure center, is located 40-50 $\mathrm{km}$ from the nascent circulation center. The arc of deep convection matches well with the area of confluence in the streamline analysis provided in Fig. 29.

In order to view the reflectivity structures in more detail, vertical cross-sections of the reflectivity structure (RHI), taken from the tail radar of the WP-3D are shown in the next two figures. Figure 31a represents a vertical cross-section displayed as the line marked "A", in Fig. 30. The RHI scan in Fig. 31a is from north to south. Notice that this scan cuts through the arc of convection located to the north of the circulation center. Echo tops in this convection reach a height of about $16 \mathrm{~km}$. In Fig. 31b, the RHI scan runs from northwest to southeast and is shown as a line marked "B" in Fig. 30. To the northwest there is an anvil with its base near $6 \mathrm{~km}$ and tops around $10 \mathrm{~km}$. The area of stratiform clouds (anvil) is the exhaust from the convective arc that lies to the north of the circulation center. The WP-3D in Fig. 31b is located about $10 \mathrm{~km}$ northwest of the low level circulation center at the time of the RHI scan. To the southeast of this scan, the reflectivity rates are about 20 to $30 \mathrm{dBZ}$. This represents a rain rate of about 1-4 mm per hour. The area of light rain is located directly over the low-level circulation center. 
Previous research has confirmed that TCs form from a pre-existing disturbance. The inner core of the cyclone is thought to evolve from a MCV that is associated with a pre-existing mesoscale area of thick stratiform clouds (Bosart and Sanders 1981, Velasco and Fritsch 1987, Miller and Fritsch 1991, Laing and Fritsch 1993a,b, Fritsch et al. 1994, Harr et al. 1996, Ritchie and Holland 1997, Simpson et al. 1997, Bister and Emanuel, 1997, Rodgers and Fritsch 2001, Montgomery et al. 2006). The streamline analysis at 5 km (Fig. 32) shows that an MCV has already formed with its center located about $50 \mathrm{~km}$ north-northeast of the low-level circulation center and just south of the convective arc. There has been speculation as to the role of the MCV in genesis. In the Tropical Experiment in Mexico (TEXMEX) studies, Bister and Emanuel (1997) suggest that after an MCS and a mid-tropospheric MCV has formed, the troposphere becomes nearly saturated on the mesoscale in the vortex core. This allows for an increase in the midtropospheric moist static energy so that evaporation of rain does not generate downdrafts that bring cool dry air to the BL. Bister and Emanuel (1997) hypothesize that the MCV is advected towards the surface and enhances surface fluxes due to an increase in the surface winds. After convection occurs, the temperature of the vortex core will increase, and the WISHE theory can then act as a positive feedback to the warm core cyclone. Montgomery (2006) shows that VHTs concentrate vertical vorticity through a combination of stretching of both the low-level and MCV vorticity rich environment and tilting of the MCV horizontal vorticity. The warm core then forms because of VHT mergers.

In the case of Tropical Storm Humberto, the MCV is advecting the exhaust from the arc of deep convection, in a cyclonic direction over the low-level circulation center. 
The RHI scan in Fig. 31b clearly shows that an anvil is located to the northwest of the circulation center and circulates over the surface low pressure center. This is in contrast to prior work. In Humberto, the MCV is not advected to the surface; it remains at midlevels as the warm core forms under the trailing edge of the anvil. There is no evidence that the warm core is forming in an area where VHTs are merging. The convective cells are located $50 \mathrm{~km}$ north of the low-level circulation center.

\subsubsection{The Role of the Stratiform Precipitation in Forming the Warm Core}

During the Global Atmospheric Research Program Atlantic Tropical Experiment (GATE), a network of observation platforms investigated mesoscale convective systems as they moved off the coast of Africa into the Atlantic Ocean. Zipser (1977) and Houze (1977) looked at the structure of the strong squall lines. Figure 33 is a schematic of one of these squalls. The leading edge of the squall line is composed of deep convection. Just behind this leading edge is an area of heavy precipitation where cool saturated downdrafts are collocated with a meso-high pressure area. In the rear of the squall is an area of lighter stratiform precipitation where a meso-low pressure area is maintained. This meso-low is characterized by a specific type of sounding that is nearly saturated and moist adiabatic from around $600 \mathrm{hPa}$ to the tropopause and has a distinct dry adiabatic subsidence region from $600 \mathrm{hPa}$ to the top of the BL. This dry adiabatic area is characterized by warm dry air with high dew point depressions.

Leary (1980) used a one-dimensional, steady-state, hydrostatic model in an attempt to reproduce this area of dry adiabatic descent on the trailing edge of the anvil. The dark dashed line in Fig. 34 shows a sounding for a mesoscale downdraft originating at $600 \mathrm{hPa}$ with a temperature of $0^{\circ} \mathrm{C}$ and relative humidity of $100 \%$. The profiles of 
temperature and humidity in these soundings are taken from Zipser (1977) and are located under the trailing edge of a precipitating anvil cloud. Leary shows that with a realistic drop-size distribution, the model reproduces soundings that closely resemble the soundings found in the meso-low area of the tropical squall line. She found that with light rain rates ( $\sim 1-5 \mathrm{~mm}$ per hour) and a pre-existing mesoscale downdraft originating at the base of the anvil of $0.5 \mathrm{~m} \mathrm{~s}^{-1}$, the model soundings closely resembled the thermodynamic structures found in these mesoscale unsaturated downdrafts. Zipser (1977) used wind observations at 500 meters and the continuity equation to estimate mesoscale downdraft speeds of about $0.05-0.25 \mathrm{~m} \mathrm{~s}^{-1}$ in this region of the tropical squall line. Leary (1980) reproduced this result in the one-dimensional model.

Near the low-level circulation center of Humberto on the trailing edge of the anvil, from about $600 \mathrm{hPa}$ to about $850 \mathrm{hPa}$, the temperature profile is close to dry adiabatic (Fig. 35). Dew point depressions reach about $13^{\circ} \mathrm{C}$ at $820 \mathrm{hPa}$. The base of the inversion is at $940 \mathrm{hPa}$. Another GPS sonde launched near the circulation center (Fig. 36) shows the temperature and dew point through the entire troposphere. In this sounding the lapse rate is dry adiabatic from approximately $550 \mathrm{hPa}$ down to about 800 $\mathrm{hPa}$. The base of the inversion is at $930 \mathrm{hPa}$. Both of these soundings look very similar to the soundings discussed by Zipser (1977) and Leary (1980). Figure 37 shows the vertical velocity observed by the WP-3D as it travels under the stratiform area near the circulation center. The WP-3D track is from west southwest to the east northeast and the two black dots show in Fig. 30 show its position relative to the circulation center circulation. A downdraft of significant magnitude is present during the passage near the circulation center. The magnitude of the downdraft is 2 to $6 \mathrm{~m} \mathrm{~s}^{-1}$ for about 3 minutes 
corresponding to a distance of $25 \mathrm{~km}$. The area of mesoscale descent is most likely even larger in scale as the WP-3D flew adjacent to the circulation center.

I compare the two soundings found near the surface circulation center with soundings jettisoned at the periphery of the analysis area to determine the impact of the warm dry layer that was apparent from $500 \mathrm{hPa}$ to $1000 \mathrm{hPa}$ on the surface pressure. Calculating the hydrostatic pressure deficit from this warm dry layer reveals that this layer accounts for about a 9 to $11 \mathrm{hPa}$ drop in pressure, depending on which peripheral sounding is used. This accounts for over $90 \%$ of the total pressure drop observed at the surface in Tropical Storm Humberto.

Using all the GPS sondes jettisoned on the $22^{\text {nd }}$, a three-dimensional matrix is constructed and then a vertical cross-section of the temperature perturbation is made as described in section 3.3.1. The temperature perturbation cross-section (Fig. 38) shows that there is a maximum temperature perturbation of $6-7^{\circ} \mathrm{C}$ located at about $2 \mathrm{~km}$. The low-level temperature perturbation is found beneath the anvil with rain rates of approximately 1-4 mm per hour. This suggests that in Tropical Storm Humberto, conditions occur that are similar to those discussed by Zipser (1977) and Leary (1980) except that the magnitude of the downdraft from observations just below the anvil is larger.

Subsidence initiated by the trailing edge of the stratiform precipitation forms a low-level warm core and decreases the surface pressure. In the upper troposphere thermodynamic conditions are similar to the surrounding environment with no evidence of the expected positive temperature perturbation that has been observed in mature hurricanes (e.g., Hawkins and Imbembo 1976). There is only slight warming over the 
circulation center with a small positive temperature perturbation of about $1^{\circ} \mathrm{C}$ (Fig. 39). One might expect some warming to the north and the possibility of a tilted warm core because of the moderate VWS, however this is not present. In these early stages of development when Humberto is a tropical storm, the maximum temperature perturbation is not in the upper atmosphere as would be expected from the WISHE theory.

\subsubsection{Equivalent Potential Temperature Increase beneath the Nascent Warm Core}

The plan view of $\theta_{\mathrm{e}}$ at 20 meters (Fig. 40) exhibits high values collocated with the circulation center. On the periphery of the analyzed field $\theta_{\mathrm{e}}$ is between 340 and $350 \mathrm{~K}$ at 20 meters. Moving closer to the circulation center, there is a sharp gradient as $\theta_{\mathrm{e}}$ increases from 350 to $365 \mathrm{~K}$ under the nascent eye with its maximum value just a few kilometers east of the low-level circulation center. The vertical cross-section in Fig. 41 displays a dome of high $\theta_{\mathrm{e}}$ located around the surface circulation center. Values of $\theta_{\mathrm{e}}$ above $350 \mathrm{~K}$ reside below about $1.5 \mathrm{~km}$ and values between 355 and $360 \mathrm{~K}$ are located below $750 \mathrm{~m}$. Fifty $\mathrm{km}$ to the north, elevated values of around $347 \mathrm{~K}$ are found above 2 $\mathrm{km}$ and are collocated with the arc of deep convection north of the circulation center, displayed in Fig. 30.

The area of mesoscale descent located on the trailing edge of the anvil shown in the soundings in Figs. 35 and 36 observed from the WP-3D (Fig. 37) is performing two functions. It is responsible for the formation of the nascent warm core located in the lower levels of the troposphere (Fig. 38). The area of mesoscale descent is also capping the area beneath it, which allows for a buildup of high energy air via fluxes from the sea surface. 
Figure 42 shows $\theta_{\mathrm{e}}$ observed by a U.S. Air Force reconnaissance flight between 200 and $500 \mathrm{~m}$ for TC Danny, which developed in a moderate shear environment (Molinari et al. 2004). The flights are radial legs through the low-level circulation center. Figures $42 \mathrm{a}$ and $\mathrm{b}$ are tropical depression and early tropical storm stage. Figure $42 \mathrm{c}$ is during the late tropical storm stage. The $\theta_{\mathrm{e}}$ distribution is very similar to Humberto (Fig. 41) with little or no change in $\theta_{\mathrm{e}}$ outside the RMW and a rapid increase in $\theta_{\mathrm{e}}$ inside the RMW. The WISHE signature of higher $\theta_{\mathrm{e}}$ collocated with increasing wind speeds is not evident.

The cap (Fig. 43), has values of CIN as low as $-250 \mathrm{~J} \mathrm{~kg}^{-1}$ over the low-level circulation center. CAPE near the low-level circulation center is as high as $2500 \mathrm{~J} \mathrm{~kg}^{-1}$. High CAPE values of $\sim 2000 \mathrm{~J} \mathrm{~kg}^{-1}$ are also found in a small area collocated with the deep convective band to the northeast of the circulation center. The most unstable boundary layer (BL) air is collocated with the circulation center and is trapped under the large cap caused by the region of subsidence shown in Figs. 35, 36 and 37. If tropical storms have large CAPE present near their center then one can imagine that the accelerating updraft composed of this air can contribute to spin-up via the stretching term $((\zeta+\mathrm{f}) \partial \mathrm{w} / \partial \mathrm{z}$, also known as the divergence term) in the absolute vorticity equation. This is the dominant term in the spin-up process given that the environment of a developing TC has low vertical shear of the horizontal wind (McBride and Zehr 1981) and density variations (solenoidal term) are negligible.

\subsubsection{Evolution of Reflectivity Structures and Building an Eyewall}

Figure 44 is a plan view of the reflectivity structure at 1942 UTC. The WP-3D is flying from north to south at an altitude of $5 \mathrm{~km}$. Comparing Fig. 44 to Fig. 30 which 
was taken at 1900 UTC, there is a marked change in the reflectivity structure. In contrast to Fig. 30, the reflectivity structure in Fig. 44 shows reflectivity as high as $46 \mathrm{dBZ}$ adjacent to the north side of the capping inversion near the circulation center. The arc of deep convection $50 \mathrm{~km}$ to the north of the circulation center looks less organized at this time. Figure 45 is a graph of $\theta_{\mathrm{e}}$ taken from the WP-3D. At 1941 UTC the WP-3D is flying directly through the new convection on the north side of the circulation center. Equivalent potential temperatures as high as $356 \mathrm{~K}$ are recorded by the WP-3D. These values correspond to the high $\theta_{\mathrm{e}}$ values located under the nascent eye. Since $\theta_{\mathrm{e}}$ is conserved following a parcel assuming no mixing or diabatic processes, these high values are sourced from the BL beneath the nascent eye. Therefore, the inflow into the new convection is coming from under the nascent eye. As the WP-3D flies south it encounters another area of warm $\theta_{\mathrm{e}}(350 \mathrm{~K})$ at $1942 \mathrm{UTC}$. The $350 \mathrm{~K} \theta_{\mathrm{e}}$ is advected cyclonically around the nascent eye and mixing out from the strong convection on the north side of the nascent eyewall.

The plan view of the reflectivity at 2109 UTC (Fig. 46) shows that the arc of convection in Fig. 30 still exists and contains cells which continue to thrive to the east and southeast of the circulation center. At this time the WP-3D is far enough away from the circulation center that attenuation is a problem when viewing the convection near the storm center. The plan view of the reflectivity at 2123 UTC (Fig. 47) reveals that the convection near the circulation center has now changed dramatically. Instead of two distinct areas of convection, the arc located $50 \mathrm{~km}$ to the north of the circulation center (Fig. 30) and newly formed convection just to the north and east of the center (Fig. 44), the two convective areas have now re-formed into one broad arc of convection. In 
observing a loop of the radar images, at the time that high $\theta_{\mathrm{e}} \mathrm{BL}$ air breaks out from under the nascent warm core and initiates convection just north of the circulation center, the arc of convection $50 \mathrm{~km}$ to the north breaks apart and new convective cells start to form adjacent to the nascent eyewall, forming a large wedge shape that I think is the new eyewall with the trailing southeast portion of the convective arc remaining as a major rainband.

\subsection{Energy Budget for the Warm $\theta_{\mathrm{e}}$ in the Eye}

A closer look at Figs. 40 and 41 evokes a critical question; how does such a large increase in the energy content occur on trajectories towards the nascent eye? To test whether energy fluxes from the sea surface are enough, an energy budget is calculated. Different trajectories are investigated because of the lack of data southeast of the lowlevel circulation center. There are 3 trajectories in Fig. 48 marked "A", "B", and " $C$ ". Trajectory "A" starts from southeast of the circulation center and ends near the center of high $\theta_{\mathrm{e}}$. Trajectory " $\mathrm{B}$ " initially feeds the convection $50 \mathrm{~km}$ north of the circulation center and is associated later with lower $\theta_{\mathrm{e}}$ on the northwest side or outflow portion of the convection. Trajectory "B" splits into two paths, "B2" spirals into the circulation center from the south and "B1" continues to the southeast and never enters the circulation center. Either of these two paths is possible because the lack of data to the southsoutheast of the circulation center does not allow confirmation of which path is actually occurring. Trajectory "C" starts from northwest of the low-level circulation center and moves to the south but the air in " $C$ " never enters the circulation center. Trajectories " $A$ " and "B2" will be investigated in the following sections. 


\subsubsection{Reflectivity Structure along the Inflow Path " $B$ "}

The plan view of reflectivity (Fig.49) displays an image of Tropical Storm Humberto as the WP-3D first flew into the storm at 1858 UTC. Superimposed on this image is the approximate deep layer inflow path that I analyze, marked as "B2" in Fig. 48. A close look at Fig. 49 shows that along the inflow path starting just northwest of the circulation center there is no convection. The plan view of reflectivity near the end of the experiment (Fig. 47) has changed from 1858 UTC (Fig. 49). Even though Humberto's reflectivity has evolved, there is no convection to the west and south of the storm in the vicinity of the inflow path. The reflectivity field along the inflow path confirms that no convective transports of energy are occurring that would remove energy from the inflow. Also of note is that the area that is examined is capped, as shown in Fig. 43.

\subsubsection{Low Level Kinematic and Thermodynamic Structures}

Following the procedures described in the methods section, 3 soundings are taken along a deep layer inflow column. Figure 50 displays a plan view of the $10 \mathrm{~m} \theta_{\mathrm{e}}$. The dashed line shows the inflow path and the 3 sounding positions, which are marked by an "X". The surface $\theta_{\mathrm{e}}$ content changes by less than $5 \mathrm{~K}$ from sounding 1 to 2 . From sounding 2 to 3 there is an increase in $\theta_{\mathrm{e}}$ of over $15 \mathrm{~K}$. I investigate the change in energy along the inflow path in between each of the soundings and compare it with the total surface fluxes from the sea to ascertain if balance can be achieved, essentially determining if the chosen inflow trajectory is possible.

Sea surface fluxes along the inflow path will be calculated using the bulk aerodynamic formulas which are an approximation for the turbulent vertical transport term $\left(\partial\left(w^{\prime} \theta_{e}\right)^{\prime} / \partial z\right)$ in equation (12) for the surface layer. To obtain an accurate 
measurement of the turbulent fluxes from the sea surface, the SST's are obtained from the AXBTs (Fig. 27), observations of the $10 \mathrm{~m}$ temperature, specific humidity, and ground relative total wind speed are from the GPS sondes (Figs. $51-53$ ). An approximate estimate of these values along each segment of the inflow path will be used to calculate the fluxes from the air-sea interface.

The Stanton number (heat transfer coefficient, $\mathrm{C}_{H}$ ) and the Dalton number (moisture transfer coefficient, $\mathrm{C}_{q}$ ) are taken from guidance by Large and Pond (1982). I also compare the moisture and heat transfer coefficients with those from Fairall et al. (2003). The uncertainty of the Large and Pond (1982) measurements is about $20 \%$. Measurements for $\mathrm{C}_{H}$ and $\mathrm{C}_{\mathrm{q}}$ are not greater than $23 \mathrm{~m} \mathrm{~s}^{-1}$ and $14 \mathrm{~m} \mathrm{~s}^{-1}$ respectively. The average wind speeds observed along the inflow trajectory in Humberto are approximately 17-18 $\mathrm{m} \mathrm{s}^{-1}$. These fall within range of reliably estimated $\mathrm{C}_{H}$ and are just a few m s above the observed range for $\mathrm{C}_{q}$.

\subsubsection{Surface Layer Flux and Divergence}

The divergence along the inflow path (Fig. 54) from sounding 1 to 2 (Fig. 50) is calculated as described in section 3.7.1. Starting from sounding 1, from 0 to approximately $25 \mathrm{~km}$, there is minimal divergence, an order of magnitude less than the divergence from 30 to $70 \mathrm{~km}$. From $30 \mathrm{~km}$ to $70 \mathrm{~km}$, convergence increases from approximately $-1.0 \times 10^{-4} \mathrm{~s}^{-1}$ to a maximum of $-5.0 \times 10^{-4} \mathrm{~s}^{-1}$ in the layer centered at 600 meters. The profiles of $\theta_{\mathrm{e}}$ for each of the 3 soundings (Fig. 55) show a progressive increase of $\theta_{\mathrm{e}}$ moving closer to the surface circulation center. There is a layer in sounding 1 (Fig. 55) from 0 to 400 meters where $\theta_{\mathrm{e}}$ is reasonably well mixed. The divergence present along the inflow path will cause this layer to be squeezed and grow to 
a higher height. From equation (13) the vertical velocity at the top of this layer can be calculated.

I start with the top of the $400 \mathrm{~m}$ layer and calculate the vertical velocity at $14 \mathrm{~km}$ intervals along the inflow path. This allows for a more realistic view of how the layer grows in time. Having calculated the vertical velocity at each of the 9 layers (100-1000 $\mathrm{m}$ ) and using a linear interpolation to find the vertical velocity in between these layers I can accurately calculate the upward progression of the layer as it rises at approximately $14 \mathrm{~km}$ intervals along the first inflow trajectory. Using this method, the layer increases by $635 \mathrm{~m}$ and therefore reaches a height of 1,035 m. The dashed line in Fig. 55 connected to sounding 1 shows how the convergence changes the vertical profile of $\theta_{\mathrm{e}}$ from sounding 1 to 2 .

Calculating the total change in energy from sounding 1 to 2 , but now with the new profile, gives a value of $3.36 \times 10^{6} \mathrm{~J} \mathrm{~m}^{-2}$. This equates to an average energy uptake of $526 \mathrm{~W} \mathrm{~m}^{-2}$ from the sea surface. Using $\mathrm{C}_{H}$ and $\mathrm{C}_{q}$ from Large and Pond (1982), where $\mathrm{C}_{H}$ is $1.3 \times 10^{-3}$ and $\mathrm{C}_{q}$ is $1.4 \times 10^{-3}$, an $\mathrm{SST}$ of $28.4^{\circ} \mathrm{C}$, an average $10 \mathrm{~m}$ air temperature of $24^{\circ} \mathrm{C}$, an average specific humidity of $17 \mathrm{~g} \mathrm{~kg}^{-1}$, and a ground relative wind speed of $18 \mathrm{~m} \mathrm{~s}^{-1}$ (Figs. 27, 51-53), I calculate an estimate of the surface fluxes from the bulk aerodynamic equations. The sensible heat flux equals $104 \mathrm{~W} \mathrm{~m}^{-2}$ and the moisture flux equals $453 \mathrm{~W} \mathrm{~m}^{-2}$. This gives a total energy uptake of $557 \mathrm{~W} \mathrm{~m}^{-2}$ from the sea surface. This is within about $6 \%$ of the actual energy gained and essentially balances equation (12). I also calculate the air-sea fluxes using the updated Fairall et al. (2003) algorithm, where $\mathrm{C}_{H}$ is $1.15 \times 10^{-3}$ and $\mathrm{C}_{q}$ is $1.2 \times 10^{-3}$ for $18 \mathrm{~m} \mathrm{~s}^{-1}$ winds. Using the same SSTs, 10 $\mathrm{m}$ air temperature, and specific humidity, the total heat flux equals $89 \mathrm{~W} \mathrm{~m}^{-2}$ and the 
moisture flux is $386 \mathrm{~W} \mathrm{~m}^{-2}$. This gives a total flux from the sea surface of $475 \mathrm{~W} \mathrm{~m}^{-2}$. The difference using Fairall et al. (2003) is slightly larger and is approximately $10 \%$. Assuming that additional energy from sea spray is negligible due to the low wind speeds and entrainment at the top of the layer is minimal, the flux calculations match well with the calculated energy increase from the GPS sondes.

A similar method is used to compare the energy balance from sounding 2 to 3 (Fig. 50). Because of the large energy increase along this inflow path, I use the larger values of $\mathrm{C}_{H}$ and $\mathrm{C}_{q}$ from Large and Pond (1982). Calculating the total change in energy from sounding 2 to 3 gives a value of $15.7 \times 10^{6} \mathrm{~J} \mathrm{~m}^{-2}$ over a distance of $75 \mathrm{~km}$ using a wind speed of $17 \mathrm{~m} \mathrm{~s}^{-1}$. This equates to an average energy uptake of $3,554 \mathrm{~W} \mathrm{~m}^{-2}$ from the sea surface. Again using $\mathrm{C}_{H}$ and $\mathrm{C}_{q}$ from Large and Pond (1982), an SST of $28.4^{\circ} \mathrm{C}$, an average $10 \mathrm{~m}$ air temperature of $26^{\circ} \mathrm{C}$, and an average specific humidity of $18 \mathrm{~g} \mathrm{~kg}^{-1}$, the bulk aerodynamic equations estimate a sensible heat flux of $65 \mathrm{~W} \mathrm{~m}^{-2}$ and a moisture flux of $407 \mathrm{~W} \mathrm{~m}^{-2}$. This gives a total energy uptake of $472 \mathrm{~W} \mathrm{~m}^{-2}$. Even if the convergence is large enough to push the lowest $100 \mathrm{~m}$ layer up (sounding 2) until it intersects sounding 3 , the average energy uptake from the sea surface is $2,201 \mathrm{~W} \mathrm{~m}^{-2}$. There is no way that the surface fluxes can account for the observed increase of energy of the inflow column.

The fact that the sub-grid scale surface fluxes in equation (12) are inadequate to balance the grid-scale advection of $\theta_{\mathrm{e}}$ and the divergence term for the second inflow trajectory (sounding 2 to 3 ) demonstrates that pathway B2 is impossible. The air on trajectory "B2" never enters the core and instead follows the path labeled "B1". 
Obviously the air that enters into the nascent eye region must come from another source; this trajectory is marked as " $\mathrm{A}$ " in Fig. 48 and is investigated in the next section.

\subsubsection{Investigation of Trajectory " $A$ "}

Path "A" shows the flow for a trajectory entering the circulation center from the southeast. SSTs average $28.3^{\circ} \mathrm{C}, 10 \mathrm{~m}$ wind speeds average $11 \mathrm{~m} \mathrm{~s}^{-1}, 10 \mathrm{~m}$ temperatures average $26.5^{\circ} \mathrm{C}$ and the average $10 \mathrm{~m}$ specific humidity is $19.5 \mathrm{~g} \mathrm{~kg}^{-1}$. The energy increase in the column, the divergence along the trajectory, and the time it takes for air to reach the center are calculated from the derived fields.

The initial, initial corrected for divergence, and final soundings for pathway " $A$ " are depicted in Fig. 56. The divergence is estimated with the radial wind component along the circumference of the circle with a $25 \mathrm{~km}$ radius. The magnitude of the radial flow is calculated every $20 \mathrm{~km}$ around the circle. The radial flow components are determined for altitudes of 50,100, 200,500, and $1000 \mathrm{~m}$. The net mass flux into the 25 $\mathrm{km}$ ring is estimated for different layers and the divergence is estimated from the total mass within the layer. Similar to the previous section, the vertical velocity is calculated based on the divergence in each layer. The divergence for the lowest $200 \mathrm{~m}$ is $-2.2 \times 10^{-}$ ${ }^{4} \mathrm{~s}^{-1}$ which gives a vertical velocity of $4.4 \times 10^{-2} \mathrm{~m} \mathrm{~s}^{-1}$. The divergence for the $500 \mathrm{~m}$ layer is $-1.2 \times 10^{-4} \mathrm{~s}^{-1}$ and results in a vertical velocity of $6 . \times 10^{-2} \mathrm{~m} \mathrm{~s}^{-1}$. Vertical velocity rates of 4.4 to $6 . \mathrm{x}^{-2} \mathrm{~m} \mathrm{~s}^{-1}$ are applied over the time it takes for the air to spiral into the circulation center, which is approximately 7 hours. The $200 \mathrm{~m}$ layer rises over $1100 \mathrm{~m}$ while the $500 \mathrm{~m}$ layer rises approximately $1500 \mathrm{~m}$. The vertical velocity rates are applied to the initial $\theta_{\mathrm{e}}$ profile to construct the new sounding adjusted for the convergence (Fig. 56). The yellow shaded region marks the amount of $\theta_{\mathrm{e}}$ needed from the fluxes from the 
sea surface to account for the change in energy along path " $\mathrm{A}$ ". The amount of energy that is needed from the corrected initial sounding to the final sounding is approximately $6.27 \times 10^{6} \mathrm{~J} \mathrm{~m}^{-2}$.

The sea surface fluxes are calculated following the scheme discussed by Wroe and Barnes (2003) with the updated Fairall et al. (2003) transfer coefficients. To estimate the fluxes the path is broken into $45 \mathrm{~km}$ segments. The combined sensible and latent heat fluxes give an approximate value of $230 \mathrm{~W} \mathrm{~m}^{-2}$. To supply the needed $6.27 \times 10^{6} \mathrm{~J} \mathrm{~m}^{-2}$ it would take just over 7.5 hours. This is within $10 \%$ of the time estimated for air to spiral into the circulation center. This essentially balances the energy equation with two assumptions; that entrainment losses through the top of the layer and gains from sea spray are negligible.

The formation of the high $\theta_{\mathrm{e}}$ dome under the nascent warm core produces the large instability and acts as the "ignition" for the nascent eyewall convection. This important step in the formation of TC Humberto has similarities with observations discussed by Molinari et al. (2004) of the $\theta_{\mathrm{e}}$ distribution during the TS stage. The high $\theta_{\mathrm{e}}$ dome is not collocated with the highest winds as would be expected from a simplified axisymmetric view of an intensifying TC but in the lower wind core of the storm. The energy budget demonstrates that the capping of the BL, the unusual $\theta_{\mathrm{e}}$ profiles with warmer $\theta_{\mathrm{e}}$ near the sea surface (Barnes 2008), and the divergence in the core of a TS are all vital ingredients in the creation of the high $\theta_{\mathrm{e}}$ dome. 


\subsection{Storm-scale Structures during the Three Days of the Experiment and their Relationship to the Environmental Wind Shear}

Fundamental structures are shown to better understand the kinematic and thermodynamic structures for a high latitude TC evolving in a highly sheared environment. Many of the structures shown are asymmetric and differ from the low latitude more symmetric fields of mature TCs. The reflectivity fields show similarities to past observations and modeling studies of TCs in sheared environments. The low-level kinematic and thermodynamic fields exhibit persistent relationships with the environmental shear vector.

\subsubsection{Evolution of Reflectivity Fields}

The convective structure of the storm is shown first in order to orient the reader. The structures will be discussed in the context of the environmental shear vector. Later in the text, the reflectivity will be referenced with respect to other storm scale variables.

The plan views of reflectivity in Fig. 57 are centered in the vicinity of the eyewall for each of the 3 days. The 3 images chosen for each day represent the approximate beginning, middle, and end of the WP-3D flights through the circulation center. On the $22^{\text {nd }}$ there is only $1 \mathrm{WP}-3 \mathrm{D}$ and it is flying at approximately $5 \mathrm{~km}$. The rest of the reflectivity images are from the lower WP-3D, flying at approximately $2 \mathrm{~km}$. Figs. 57a, b, and c display the evolution of the reflectivity at 1900, 1938, and 2123 UTC on the $22^{\text {nd }}$. As discussed in section 4 , there are major changes in the structure of the convection during these times. In Fig. 57a, the major feature is an arc-shaped convective band about $50 \mathrm{~km}$ north of the circulation center. By 1938 UTC, new convection has developed just north of the circulation center forming the nascent eyewall. At 2116 UTC, the nascent 
eyewall and the arc of convection have reformed into a large wedge shape. Section 4 covers in depth the thermodynamic processes occurring.

The eyewall on the $23^{\text {rd }}$ is crescent-shaped, when Humberto is a category 2 , its peak intensity for the experiment over the 3 days of sampling (Figs. 57d, e, f). As shown in other studies (Jones 1995, Bender 1997, Drury and Evans 1998, Frank 1998, Black et al. 2002, Eastin et al. 2005, Frank and Ritchie 1999, Frank and Ritchie 2001), the convection is downshear left of the shear vector. The asymmetric convective structure may be due to shear-induced differential vorticity advection with height (Frank and Ritchie, 2001). Of all 3 days, the eyewall reflectivity on the $23^{\text {rd }}$ varies the least in shape and size.

On the $24^{\text {th }}$ at 2128 UTC (Fig. 57g) the eyewall appears as two arc shaped wedges disconnected from each other to the northeast and northwest of the circulation center. At 2259 UTC (Fig. 57i) the shape of the eyewall reflectivity has changed dramatically. There appears to be almost a $90^{\circ}$ angle formed from two lines of convection with one covering the northern sector of the eyewall and one covering the eastern sector. At 2430 UTC the reflectivity in the eyewall has become more circular and forms a large arc from the northwest to the southeast of the circulation center. The eyewall is going through a period of major restructuring on the $24^{\text {th }}$, however the convection continues to be located downshear left of the shear vector.

The RHI scans in Figs. 58a, b, c, d, e, and f show the structure of convection near the circulation center. Figure 58a is a RHI scan from north to south at 1853 UTC on the $22^{\text {nd }}$. The scan runs through the arc of convection $50 \mathrm{~km}$ to the north of the circulation center. The highest reflectivity in the arc is $45 \mathrm{dBZ}$ and has cloud tops that reach about 
$16 \mathrm{~km}$. At this time, convection is not occurring close to the circulation center. The east to west vertical cross-section (Fig. 58b) displays the reflectivity at about the time the nascent eyewall first occurs. It shows reflectivity of about $45 \mathrm{dBZ}$ occurring $25 \mathrm{~km}$ north of the circulation center. The highest echo tops reach $14 \mathrm{~km}$. Returns of $37 \mathrm{dBZ}$ reach about $6.5 \mathrm{~km}$. The stratiform exhaust from the convective arc $50 \mathrm{~km}$ to the north is still clearly visible to the west of the nascent convection.

On the $23^{\text {rd }}$ at 2328 UTC (Fig. 58c) the RHI scan is from south-southeast to northnorthwest. This RHI scan shows the mature eyewall of Humberto at its peak intensity. Cloud tops reach $16 \mathrm{~km}$ with peak reflectivity of about 40-44 dBZ. High reflectivity of $37 \mathrm{dBZ}$ reaches about $8 \mathrm{~km}$. Similar to Black et al. (2002), the tallest echo tops are located in the downshear left quadrant. At 2326 UTC on the same day, Fig. 58d displays a north to south RHI scan through some of the highest reflectivity in the eyewall (see Fig. 57f). Reflectivity from $40-51 \mathrm{dBZ}$ is located from the surface to approximately $6 \mathrm{~km}$.

Figures $58 \mathrm{e}$ and $\mathrm{f}$ are at 2128 and 2428 UTC on the $24^{\text {th }}$ of the eyewall of Humberto after a period of weakening. At 2128 UTC, the RHI scan runs from west to east through the two wedges of convection shown in Fig 57g. Echo tops reach $14 \mathrm{~km}$, however the highest returns of $37 \mathrm{dBZ}$ reaches only about $4 \mathrm{~km}$. At $2428 \mathrm{UTC}$ the RHI scan runs from south-southeast to north-northwest. The echo tops, associated with a period in which the eyewall had a more circular shape and seemed to be better organized, reach about $14 \mathrm{~km}$. Scattered higher returns of $33 \mathrm{dBZ}$ make it up to $4 \mathrm{~km}$.

The highest echo tops on the $22^{\text {nd }}$ in the nascent eyewall reach $14 \mathrm{~km}$. On the $23^{\text {rd }}$, in the mature eyewall they reach $16 \mathrm{~km}$ and on the $24^{\text {th }} 14 \mathrm{~km}$. The highest echo tops of the 3 days in the eyewall occur on the $23^{\text {rd }}$ in the mature eyewall. Comparing the 
$37 \mathrm{dBZ}$ return rate, the nascent eyewall on the $22^{\text {nd }}$ has returns up to about $6.5 \mathrm{~km}$, the $23^{\text {rd }}$ has returns up to about $8 \mathrm{~km}$, and the $24^{\text {th }}$ shows returns only up to about $4 \mathrm{~km}$. The eyewall on the $23^{\text {rd }}$, when Humberto is at its peak intensity, has both the highest echo tops and the highest $37 \mathrm{dBZ}$ contours.

\subsubsection{Comparison of Eyewall Radius}

The radius of the eyewall is calculated based on the peak reflectivity in the convection. On the $22^{\text {nd }}$ at 1942 UTC as the nascent eyewall first appears, the radius of the eyewall is $25 \mathrm{~km}$ (Fig. 57). On the $23^{\text {rd }}$ the eyewall remains asymmetric (Fig. $57 \mathrm{~d}$, e, and f) and the eyewall radius stays constant at approximately $36 \mathrm{~km}$. On the $24^{\text {th }}$, there are large changes in eyewall structure throughout the period of observation. The eyewall in Fig. $57 \mathrm{~g}$ is so erratic and unorganized that a measurement of the eyewall radius is not made. The eyewall radius at 2259 UTC (Fig. 57i) is approximately $42 \mathrm{~km}$. From 00:00 UTC to 00:30 UTC on the $25^{\text {th }}$, a much wider arc appears, the eyewall extends from the northwest to southeast of the circulation center, and the eyewall radius enlarges to $54 \mathrm{~km}$.

Studies have linked the contraction of eyewalls to intensification processes

(Jordan 1961; Holliday and Thompson 1979; Willoughby et al. 1982; Jorgensen 1984a, b; Weatherford and Gray 1988; Bosart et al. 2000). The eyewall increases in size from the $22^{\text {nd }}$ to $23^{\text {rd }}$ to the $24^{\text {th }}$ despite the intensity changes. There is also an increase in eyewall size on the $24^{\text {th }}$ during the $\sim 4$ hours of sampling.

\subsubsection{Low-level Kinematic Fields}

On the $22^{\text {nd }}$ at $50 \mathrm{~m}$ (Fig. 59a), the tangential wind field has an asymmetric pattern. Peak tangential winds of $24 \mathrm{~m} \mathrm{~s}^{-1}$ are located west and northwest of the circulation center downwind of the convective arc, $50 \mathrm{~km}$ to the north. At 1,500 $\mathrm{m}$ (Fig. 
$59 \mathrm{~b}$ ) the tangential wind maximum has veered and is collocated with the arc of convection $50 \mathrm{~km}$ to the north northeast of the circulation center. Both the $50 \mathrm{~m}$ and $1,500 \mathrm{~m}$ tangential wind maxima are located left of the shear vector. On the $23^{\text {rd }}$, near the core at $50 \mathrm{~m}$ (Fig. 59c), the strongest tangential winds of 40 to $45 \mathrm{~m} \mathrm{~s}^{-1}$ extend from south of the circulation center to the northeast and are left of the shear vector. At 1,500 m (Fig. 59d) near the RMW the highest winds are located to the east, north, and west of the storm center. Similar to Hurricane Norbert (Marks et al. 1992), the strongest tangential winds slope upwind with height. On the $24^{\text {th }}$, the tangential wind field at $50 \mathrm{~m}$ (Fig. 59e) is more disorganized than on the $23^{\text {rd }}$. Near the core the highest winds are north-northwest of the circulation center and to the left of the shear vector. At 1,500 m (Fig. 59f) the tangential winds still remain strongest in the northern part of the storm. Similar to the $22^{\text {nd }}$ and the $23^{\text {rd }}$ the strongest tangential winds slope upwind with height.

On the $22^{\text {nd }}$ at $50 \mathrm{~m}$ (Fig. 60a), about $50 \mathrm{~km}$ to the northeast of the storm center, $20 \mathrm{~m} \mathrm{~s}^{-1}$ inflow diminishes to zero under the convective arc (Fig. 57a). At 1,500 m (Fig. 60b) the overall magnitude of the inflow has decreased however the pattern is similar to the $50 \mathrm{~m}$ flow. The strongest inflow in this developing TS is located downshear left of the circulation center. On the $23^{\text {rd }}$, at $50 \mathrm{~m}$ (Fig. 60c) the strongest radial winds are located near the RMW and are aligned with the shear vector. The inflow is collocated with the downshear quadrant and the outflow with the upshear quadrant. At 1,500 m (Fig. 80d), the radial winds continue to align with the shear vector. Just northwest of the circulation center in the vicinity of the RMW the strong inflow-outflow couplet is due to convective scale features. Similar to the $23^{\text {rd }}$, the $24^{\text {th }}$ has the strongest inflow-outflow couplet aligned with the shear vector at 50 and $1,500 \mathrm{~m}$. Also similar to the $23^{\text {rd }}$ the 
radial winds increase moving towards the circulation center and decrease with height. The relationship of the VWS vector to the radial wind distribution supports the results that asymmetric BL flows under higher VWS (on $23^{\text {rd }}$ and $24^{\text {th }}$ ) align with the shear vector (Bender 1997, Frank and Ritchie 1999, Frank and Ritchie 2001, Corbosiero and Molinari 2003) and with storm motion (Shapiro 1983).

\subsubsection{Divergence in the Boundary Layer}

The divergence in the eyewall region at $200 \mathrm{~m}$, a proxy for the mean BL flow, appears in Fig. 61. Divergence is calculated similar to section 4.3.4, however each circle encompassing the eyewall is divided into 8 sections. On the $22^{\text {nd }}$, convergence is located in the northern half of the eyewall and divergence in the southern half. The convergence is located to the left of the VWS vector with divergence to the right of the vector. The BL convergence is upwind of the nascent eyewall convection in Fig. 44 and 57b. On the $23^{\text {rd }}$, convergence increases its annular extent and originates in the upshear right quadrant, increasing to its highest magnitude in the downshear left quadrant. The convergence is located upwind of the convection in Figs. 57 d,e,f. On the $24^{\text {th }}$, the convergence lessens slightly in its annular extent from the $23^{\text {rd }}$, and originates in the downshear right quadrant of the storm. Similar to the $22^{\text {nd }}$ and the $23^{\text {rd }}$, convergence is located upwind of the convection in Figs. 57g,h,i. On all 3 days the convergence originates upwind of the reflectivity. Convergence covers more area around the eyewall as the storm intensifies and has a persistent relationship with the VWS and motion vector.

Intriguingly, the $23^{\text {rd }}$ has convergence initiating in the upshear quadrant of the storm. This is contrary to the idea that upward motions in the eyewall are caused by the differential advection of vorticity with height as shown by Frank and Ritchie (2001). 


\subsubsection{Low-level Equivalent Potential Temperature}

Figures $62 \mathrm{a}, \mathrm{b}, \mathrm{c}, \mathrm{d}, \mathrm{e}$, and $\mathrm{f}$ display plan views of $\theta_{\mathrm{e}}$ for the experiment at $200 \mathrm{~m}$ and at $3 \mathrm{~km}$. On the $22^{\text {nd }}$ at $200 \mathrm{~m}(62 \mathrm{a})$, there is evidence that in the nascent eyewall $\theta_{\mathrm{e}}$ increases to the right of the shear vector and decreases to the left of the shear vector. At 3 $\mathrm{km}$ on the $22^{\text {nd }}$, the highest values of $\theta_{\mathrm{e}}(342-346 \mathrm{~K})$ are collocated with the band of convection shown in Fig. 57a and do not show any relation to the shear vector.

On the $23^{\text {rd }}$ at $200 \mathrm{~m}$ (Fig. 62c), there is an area of high $\theta_{\mathrm{e}}$ in the range of $348-355$ $\mathrm{K}$ located in a large swath to the east southeast of the circulation center. This area is collocated with the rainband. Near the eyewall, $\theta_{\mathrm{e}}$ increases to the right of the shear vector and decreases to the left of the shear vector. The higher $\theta_{\mathrm{e}}$ is advected into the eyewall updraft where the convection is initiated while lower values in the upshear quadrant suppress convection. At $3 \mathrm{~km}$ on the $23^{\text {rd }}$, the $\theta_{\mathrm{e}}$ distribution becomes more symmetric and mixes around the circulation with gradual increases towards the storm center.

On the $24^{\text {th }}$ at $200 \mathrm{~m}$ (Fig. $60 \mathrm{e}$ ) the $\theta_{\mathrm{e}}$ distribution is similar to the $23^{\text {rd }}$. In the vicinity of the eyewall, $\theta_{\mathrm{e}}$ increases to the right of the shear vector with decreasing values left of the vector. The higher $\theta_{\mathrm{e}}$ is located where convection is initiated and the lower $\theta_{\mathrm{e}}$ is associated with suppressed convection. Past observations and modeling studies performed by Black et al. (2002), Eastin et al. (2005), Frank and Ritchie (1999), and Frank and Ritchie (2001) show evidence that in moderate to high shear, eyewall convection has a quasi-stationary wavenumber-1 asymmetry in mesoscale vertical velocity and reflectivity. A mesoscale ascent maximum is located downshear with a mesoscale precipitation maximum located left of the downshear vector. The $\theta_{\mathrm{e}}$ 
distribution near the eyewall of Humberto offers evidence that in the BL increased energy content is juxtaposed with upward motion while downward motion is associated with decreases in $\theta_{\mathrm{e}}$ and suppressed convection. At outer radii, the highest $\theta_{\mathrm{e}}$ is collocated with the rainband. Similar to the $23^{\text {rd }}$, at $3 \mathrm{~km}$ on the $24^{\text {th }}$, the distribution of $\theta_{\mathrm{e}}$ becomes more symmetric with increasing values towards the circulation center.

\subsubsection{Large-scale View of CAPE}

On the $22^{\text {nd }}$, the highest CAPE of 2,500 J/kg (Fig 63a) is collocated with the nascent eye. There is also an area of CAPE of $\sim 2,000 \mathrm{~J} / \mathrm{kg}$ located in the area of confluence $50 \mathrm{~km}$ northeast of the circulation center. On the $23^{\text {rd }}$, as the VWS increases, the highest CAPE at outer radii (Fig. 63b) is located in the downshear right quadrant of the storm. This area is collocated with an area of confluence in the $50 \mathrm{~m}$ streamlines and the convection associated with the rainband. The $24^{\text {th }}$ (Fig. 63c) displays a similar pattern to the $23^{\text {rd }}$ with the highest CAPE located in the downshear quadrant. The high CAPE is feeding the rainband while lower values associated with suppressed convection exist on the upshear side of the storm. This has similarities to the results of Molinari and Vollaro (2010) where CAPE in highly sheared storms was 60\% larger in the downshear quadrants. Similar to the findings of Bogner et al. (2000), CAPE on the $23^{\text {rd }}$ and $24^{\text {th }}$ diminishes in magnitude with decreasing radial distance to the circulation center.

\subsubsection{CAPE and CIN in the vicinity of the Eyewall}

Observations and modeling studies performed by Black et al. (2002), Eastin et al. (2005), and Frank and Ritchie (2001) show evidence that in moderate to high shear, eyewall convection displays a quasi-stationary wavenumber-1 asymmetry in mesoscale vertical velocity and reflectivity. Convective-scale ascent maxima are located downshear 
with convective-scale precipitation maxima located left of the downshear vector. On the $23^{\text {rd }}$, CAPE of $700-800 \mathrm{~J} \mathrm{~kg}^{-1}$ is collocated with the downshear right quadrant of the eyewall (Fig. 64 a) with small to no CAPE collocated with the upshear quadrant of the eyewall. This is consistent with the observations of Eastin et al. (2005) that the majority of the most buoyant eyewall updrafts are located in the downshear quadrant of the eyewall. High CAPE of over $1000 \mathrm{~J} \mathrm{~kg}^{-1}$ is present in the eye of Humberto. In Fig. 64b, high CIN of up to $350 \mathrm{~J} / \mathrm{kg}$ is collocated with the eye and the upshear quadrant of the eyewall. The high CIN on the upshear side of the eyewall has the effect of suppressing convection in this part of the eyewall. Similar to the $22^{\text {nd }}$ (see section 4) the cap breaks on the downshear side of the eyewall. This area is also collocated with initiation of convection displayed in Figs. 57d, e, and f.

On the $24^{\text {th }}$, after a day of weakening, Humberto shows an increase in CAPE within the circulation center (Fig 65a). CAPE within the eyewall has increased to about $1,600 \mathrm{~J} / \mathrm{kg}$ on the $24^{\text {th }}$. Similar to the $23^{\text {rd }}$, CAPE of 600 to $800 \mathrm{~J} \mathrm{~kg}^{-1}$ is present in the downshear quadrant of the eyewall with decreased values present on the upshear side. High CIN is located over the eye and the upshear quadrants of the eyewall (Fig. 65b). This has the effect of suppressing convection in this part of the eyewall. Similar to the $23^{\text {rd }}$, there is an absence of CIN southeast and north of the circulation center, collocated with the initiation of the evolving eyewall convection on the 24th.

\subsection{Thermodynamic Structures and their Relationship to Intensity}

\subsubsection{Vertical Cross-sections of Equivalent Potential Temperature}

Both Malkus and Riehl (1960) and Emanuel (1986) correlate the intensity of a TC with the mean $\theta_{\mathrm{e}}$ of the eyewall column. The vertical cross-section of from TC Inez (Fig. 
5), shows the structure of $\theta_{\mathrm{e}}$ in a mature low-latitude TC (Hawkins and Imbembo 1976). In the eyewall column $\theta_{\mathrm{e}}$ is uniform in the vertical and axisymmetric through the height of the cross-section. Figure 6 is a vertical cross-section of $\theta_{\mathrm{e}}$ from Hurricane Allen (Jorgensen 1984). Similar to TC Inez, $\theta_{\mathrm{e}}$ is well mixed vertically through the RMW.

In Tropical Storm Humberto a dome of high $\theta_{\mathrm{e}}$ (Fig. 66a) is present beneath the low-level warm core. In the southern eyewall above the $\mathrm{BL}, \theta_{\mathrm{e}}$ is similar to environmental values and does not show a column of high $\theta_{\mathrm{e}}$ as would be expected. In the northern eyewall $\theta_{\mathrm{e}}$ is slightly higher than environmental values and decreases with height.

On the $23^{\text {rd }}$, above the BL, the profile of $\theta_{\mathrm{e}}$ (Fig. 66b) is asymmetric with higher values in the northern eyewall, where convection exists. Assuming the average $\theta_{\mathrm{e}}$ in the column extends through the height of the troposphere, the convective or northern eyewall displays about a $10 \mathrm{~K}$ average increase in eyewall $\theta_{\mathrm{e}}$ from the $22^{\text {nd }}$ to the $23^{\text {rd }}$. This would yield a $30 \mathrm{hPa}$ change in the surface pressure if an average Malkus and Riehl (1960) and Emanuel (1986) relation of $\Delta \mathrm{p} \sim 3 \Delta \theta_{\mathrm{e}}$ is used . The actual pressure drop is about $20 \mathrm{hPa}$. The southern eyewall contains an average increase of about $4 \mathrm{~K}$, yielding a pressure drop of about $12 \mathrm{hPa}$ based on the previous theoretical relationship. In later sections, the asymmetries in average eyewall $\theta_{\mathrm{e}}$ on the $23^{\text {rd }}$ are shown to be related to the eyewall temperature gradients. In the lower eye reservoir, there is a net loss of $4 \mathrm{~K}$ from the $22^{\text {nd }}$ to the $23^{\text {rd }}$ indicating that the eye has given up energy to the eyewall.

On the $24^{\text {th }}$, after a $10 \mathrm{hPa}$ increase in the surface pressure of Humberto (Fig. 66c), the vertical cross-section of $\theta_{\mathrm{e}}$ displays marked contrasts from the previous day. A fairly well mixed column of higher $\theta_{\mathrm{e}}$ is present in the eye above the BL with decreased 
values in the eyewall. Kossin and Eastin (2001) showed that in intensifying storms they often saw a peak of $\theta_{\mathrm{e}}$ in the eyewall and minimum in the eye. Soon after a storm started to fill they observed more of a monotonic $\theta_{\mathrm{e}}$ distribution. They related this to asymmetric horizontal mixing as described by Schubert et al. (1999). On the $23^{\text {rd }}$, as Humberto reaches its peak intensity for the experiment, the northern eyewall at $2 \mathrm{~km}$ does show higher values than what is found in the eye. On the $24^{\text {th }}$, after a period of weakening the horizontal distribution of $\theta_{\mathrm{e}}$ is similar to the monotonic $\theta_{\mathrm{e}}$ described by Kossin and Eastin (2001). In the low-level eye reservoir, there is about a $4 \mathrm{~K}$ decrease in $\theta_{\mathrm{e}}$ from the previous day. This demonstrates that the eye reservoir has continued to give up energy to the eyewall from the $23^{\text {rd }}$ to the $24^{\text {th }}$.

The Humberto height-radial distance cross-sections are in contradistinction to previous vertical cross-sections displayed by both Hawkins and Imbembo (1976) and Jorgensen (1984). The height- radius cross-sections which pass through the TC center shown by these authors reveal two columns of warm $\theta_{\mathrm{e}}$ that are well mixed in the vertical. In Humberto on the $22^{\text {nd }}$, there is no evidence of a tall ring of warm $\theta_{\mathrm{e}}$ surrounding the eye. On the $23^{\text {rd }}$, even though the surface pressure has dropped $20 \mathrm{hPa}$, the eyewall column shows higher $\theta_{\mathrm{e}}$ in the northern eyewall where convection is present with lower values in the eye and the southern eyewall. On the $24^{\text {th }}$, the eye column contains the highest $\theta_{\mathrm{e}}$ with the eyewall column having lower values.

\subsubsection{Warm Core and Clues to Intensification}

The intensity of a TC is directly correlated to the depth and magnitude of the warm core as it is the cause of the hydrostatic pressure deficit at the sea surface. As discussed in section 4 , the warm anomaly on the $22^{\text {nd }}$ (Fig. 67a), was found under an area 
of light stratiform precipitation. The subsidence indicated by Figs. 35, 36, and 37 is responsible for the maximum warming of 6 to $7 \mathrm{~K}$, between 1,300 and 2,700 $\mathrm{m}$. On the $23^{\text {rd }}$, as Humberto reaches its maximum intensity for the experiment, maximum warming of 7 to $9 \mathrm{~K}$ is apparent between 1500 and $5700 \mathrm{~m}$ (Fig. 67b). The warming in the upper troposphere is on the order of 4 to $5.5 \mathrm{~K}$. On the $24^{\text {th }}$, after Humberto weakened to a category 1, maximum temperature anomalies of 6 to $7 \mathrm{~K}$ are located between 2,300 and 4,500 m (Fig. 67c). In the upper troposphere, temperature anomalies of approximately 4 $\mathrm{K}$ are present. The observations of Humberto with its maximum temperature perturbation in the lower troposphere are unlike past observations of mature TCs such as Figs. 2, 3 (Hawkins and Rubsam 1968, Hawkins and Imbembo 1976).

Assuming that the contribution to the total pressure drop for each layer is hydrostatic, Figs. 68a, b, and c show the pressure perturbation for a series of $1 \mathrm{~km}$ layers for each of the 3 days. On the $22^{\text {nd }}$, the maximum pressure perturbation is about $3.5 \mathrm{hPa}$ in the 1-2 km layer. The GPS sondes show warm dry air in the eye below $5 \mathrm{~km}$ (Figs. 35 and 36), coincident with the largest pressure perturbations. Above $5 \mathrm{~km}$, the pressure perturbations are well below $1 \mathrm{hPa}$. From the surface to $5 \mathrm{~km}$ over $90 \%$ of the pressure drop has occurred. The $23^{\text {rd }}$ has a maximum pressure perturbation of over $5 \mathrm{hPa}$ in the 2$3 \mathrm{~km}$ layer. The pressure perturbation declines at a steady rate towards the top of the troposphere. Below $6 \mathrm{~km}$, over $70 \%$ of the pressure is accounted for. On the $24^{\text {th }}$, the layers below $5 \mathrm{~km}$ show the largest pressure perturbations, in the vicinity of 3 to $5 \mathrm{hPa}$. Above this level there is a decline of the pressure perturbation through the upper troposphere. Similar to the $23^{\text {rd }}$, over $70 \%$ of the pressure decrease is due to warming below $6 \mathrm{~km}$. 
The warm core maximum and the majority of the pressure falls are occurring in the lower troposphere on each of the 3 days. This hints that the resilience of the storm in the fairly hostile VWS conditions discussed earlier in section 4.1 is likely due to processes occurring in the lower troposphere.

The evolution of the warm core (Fig. 69) exhibits some unexpected results. On the $22^{\text {nd }}$, the maximum warming is $7 \mathrm{~K}$ and the majority of the warming is confined to below $5 \mathrm{~km}$. Transitioning from the $22^{\text {nd }}$ to the $23^{\text {rd }}$, the warming increases in both the lower and upper troposphere. Above $5 \mathrm{~km}$ warming increases by 4 to $5 \mathrm{~K}$. In the 2 to 3 $\mathrm{km}$ layer warming increases by 1 to $3 \mathrm{~K}$. The maximum warming on the $23^{\text {rd }}$ of over $8 \mathrm{~K}$ occurs between 2 and $3 \mathrm{~km}$. Comparing the $23^{\text {rd }}$ to the $24^{\text {th }}$, warming above $6 \mathrm{~km}$ decreases by only about $1 \mathrm{~K}$. The largest cooling is in the 4 to $6 \mathrm{~km}$ layer with a 2 to $4 \mathrm{~K}$ decrease in temperatures. Below $4 \mathrm{~km}$ there is cooling of 1 to $3 \mathrm{~K}$.

The warm core develops in the lower troposphere and builds upward from the $22^{\text {nd }}$ to the $23^{\text {rd }}$ as the storms central pressure drops $20 \mathrm{hPa}$. This is in contrast to past theories (Malkus and Riehl 1960, Emanuel 1986) and observations (Hawkins and Rubsam 1968, Hawkins and Imbembo 1976), where the highest temperature perturbations would be found in the upper troposphere due to increased fluxes from the sea surface and the spreading of moist adiabats with height. In the aforementioned theories, the maximum warming would always occur in the upper troposphere and continued intensification would show the greatest changes in the temperature perturbation at approximately $300 \mathrm{hPa}$. 


\subsubsection{Asymmetric Structure of the Warm Core on the $23^{\text {rd }}$}

A vertical cross-section through the warm core below $3.5 \mathrm{~km}$ on the $23^{\text {rd }}$ (Fig. 70) displays horizontal temperature gradients on the north side of the eye of approximately $8^{\circ}$ K over $20 \mathrm{~km}$. On the south side of the eye, the horizontal temperature gradient lessens to about $3^{\circ} \mathrm{K}$ over $20 \mathrm{~km}$. The red line marked "A" is where a GPS sonde was dropped on the inner edge of the northern eyewall (Fig. 71a). At about $600 \mathrm{hPa}$ the winds are from a westerly direction with a magnitude of $15 \mathrm{~m} \mathrm{~s}^{-1}$. At this same altitude radially out from the GPS sonde "A" wind speeds are $40 \mathrm{~m} \mathrm{~s}^{-1}$. Therefore GPS sonde "A" starts its fall inside the RMW. Following the sounding towards the surface, the winds increase quickly to $33 \mathrm{~m} \mathrm{~s}^{-1}$ at $800 \mathrm{hPa}$ and $50 \mathrm{~m} \mathrm{~s}^{-1}$ at about $950 \mathrm{hPa}$. The sounding is nearly saturated and moist adiabatic throughout the entire layer. Figure $71 \mathrm{~b}$ is the sounding marked "B" in Fig. 70 and was jettisoned in the southern eyewall. Sounding "B" is dropped in an area that is still warm with respect to the environment and it contains multiple temperature inversions. The sonde was jettisoned from a relatively low height and shows that the southern eyewall is warmer and drier than the northern eyewall in Fig. 71a. Figure 71c shows a sounding in the southwest RMW, from just west of sounding "B". Similar to Fig. 70 the sounding shows that the south side of the eyewall is warm and dry with dew point depressions as high as $10^{\circ} \mathrm{C}$ just below $700 \mathrm{hPa}$.

The increase in warming in the southern eyewall lowers the pressure hydrostatically in the southern eyewall, allowing for a balance between the high $\theta_{\mathrm{e}}$ (discussed in section 4.5.1) in the northern convective area (downshear) of the eyewall with the area of suppressed convection (upshear) in the southern eyewall. 


\subsubsection{Eye to Eyewall Mixing on the $23^{\text {rd }}$}

The plan view in Fig.72 displays a plug of warm $\theta_{\mathrm{e}}$ located within the RMW with values as high as $360 \mathrm{~K}$. The observations of $\theta_{\mathrm{e}}$ from the lower WP-3D (Fig. 73a) match well with the vertical cross section of $\theta_{\mathrm{e}}$ shown in Fig. $66 \mathrm{~b}$ constructed from the GPS sondes. The tangential winds show a clearly defined RMW with the maximum winds in the northern eyewall. Moving from south to north, $\theta_{\mathrm{e}}$ increases from about $340 \mathrm{~K}$ to 350 $\mathrm{K}$ near the southern RMW. Within the RMW $\theta_{\mathrm{e}}$ is near $353 \mathrm{~K}$ and just inside the northern part of the RMW $\theta_{\mathrm{e}}$ increases to its highest value of $\sim 358 \mathrm{~K}$ before decreasing to the north. The highest vertical velocity of $\sim 10 \mathrm{~m} \mathrm{~s}^{-1}$ is collocated with the northern RMW. This is in the top $1 \%$ of eyewall updrafts according to Black et al. (1996). The $\theta_{\mathrm{e}}$ advected upward in this updraft is about $352 \mathrm{~K}$. The highest $\theta_{\mathrm{e}}$ of $358 \mathrm{~K}$ is coincident with much smaller updrafts and downdrafts a short distance within the northern RMW. At $5 \mathrm{~km}$ (Fig. 73b) the aircraft is flying from southeast to northwest through the storm center. Similar to the $2 \mathrm{~km}$, the WP-3D observed a strong updraft of $10 \mathrm{~m} \mathrm{~s}^{-1}$ in the northwest eyewall, $\theta_{\mathrm{e}}$ of $355 \mathrm{~K}$ collocated with the updraft, and tangential winds of similar magnitude to $2 \mathrm{~km}$. The warm $\theta_{\mathrm{e}}$ air in the eye (Fig. 72) is associated with CAPE of over $1000 \mathrm{~J} \mathrm{~kg}^{-1}$ in Fig. 64a. The warm $\theta_{\mathrm{e}}$ of $358 \mathrm{~K}$ observed by the WP-3D is only found in the BL radially inward of the RMW in Fig. 72. This air may have a larger CAPE than calculated as it is ascends the northern eyewall where CIN is absent and it moves along constant angular momentum lines instead of vertically. Similar to observations by Montgomery et al. (2006), Eastin et al. (2005) and Barnes and Fuentes (2010) the eye to eyewall mixing on the $23^{\text {rd }}$ is giving an energy boost to Humberto that 
allows it to reach its peak intensity for the experiment even though half the circulation is over sub $26^{\circ} \mathrm{C} \mathrm{SSTs.}$

\subsection{Environmental Influences on the Warm Core Structure: Causes for the Unusual Warm Core Structure}

4.6.1 Warm Core Structure and Dry Air Intrusion on the $23^{\text {rd }}$

The cross-section of relative humidity (Fig. 74) reveals that in the eye, the layer from $1,500 \mathrm{~m}$ to $6,500 \mathrm{~m}$ has a lower relative humidity of $40-60 \%$ compared with the areas above and below this layer. The dry layer in the eye is associated with lapse rates that are close to dry adiabatic. In the southern eyewall there is extremely dry air with relative humidity of $10-20 \%$ encroaching into the eyewall. Air with CAPE of approximately $700 \mathrm{~J} \mathrm{~kg}^{-1}$ is collocated with the downshear side of the eyewall and CAPE of over $1000 \mathrm{~J} \mathrm{~kg}^{-1}$ is associated with eye to eyewall mixing (Fig. 64). The schematic in Fig. 75 shows the effect of dry air intrusion on an undilute updraft. Above the base level of dry air intrusion the parcel path shifts to a cooler moist adiabat as the updraft cools to its wet-bulb temperature due to entrainment.

On the $23^{\text {rd }}$ there is a steady decrease in warming above $6 \mathrm{~km}$ (Fig. 69) collocated with the intrusion of dry air into the eyewall. The WP-3D flights at 2 and $5 \mathrm{~km}$ show a persistent updraft with $\theta_{\mathrm{e}}$ of $355 \mathrm{~K}$ spiraling up the eyewall column. There were no flights between 5 and $10 \mathrm{~km}$ so a high altitude GPS sonde jettisoned in the storm center is used to estimate the $\theta_{\mathrm{e}}$ in the upper levels of the storm. Equivalent potential temperatures of between 348 and $350 \mathrm{~K}$ are located from 6 to $10 \mathrm{~km}$. This is a 5 to $8 \mathrm{~K}$ drop in $\theta_{\mathrm{e}}$ from the eyewall updraft shown in Figs. 73a,b. The theoretical and empirical arguments made by Malkus and Riehl (1960) and Emanuel (1986) did not take into to account the effect of 
dry air entrainment in the eyewall and of the possibility large vertical gradients of $\theta_{\mathrm{e}}$ in the eyewall column.

\subsubsection{Warm Core Structure and Dry Air Intrusion on the $24^{\text {th }}$}

On the $24^{\text {th }}$, CAPE of approximately $800 \mathrm{~J} \mathrm{~kg}^{-1}$ (Fig. 65a) is present on the downshear side of the eyewall. There is a layer of air with relative humidity of $40-55 \%$ from $2,500 \mathrm{~m}$ to $4,500 \mathrm{~m}$ in the eye of Humberto (Fig. 76). The dry layer in the eye is associated with lapse rates that are close to the dry adiabatic. In the southern eyewall there is dry air $(\mathrm{RH} \sim 50 \%)$ intruding into the eyewall at about the $4 \mathrm{~km}$ level.

The schematic of the difference in the temperature perturbation in the core of Humberto from the $23^{\text {rd }}$ to the $24^{\text {th }}$ (Fig. 77) shows the largest cooling of $4^{\circ} \mathrm{C}$ between 4 and $6 \mathrm{~km}$. This coincides with the lowering of the dry air intrusion from $6 \mathrm{~km}$ on the $23^{\text {rd }}$ to $4 \mathrm{~km}$ on the $24^{\text {th }}$. Similar to the $23^{\text {rd }}$, the dry air has the effect of cooling the updraft to its wet-bulb temperature as shown in Fig. 75. The warm dry air in the eye has also decreased in its vertical extent. It has lowered from about $6 \mathrm{~km}$ on the $23^{\text {rd }}$ to approximately $4 \mathrm{~km}$ on the $24^{\text {th }}$. The largest cooling is collocated with the difference between the level of dry air intrusion on the $23^{\text {rd }}(6 \mathrm{~km})$ and the $24^{\text {th }}(4 \mathrm{~km})$ shown in Figs. 74 and 76.

The cross-sections of the warm core differences and relative humidity offer compelling evidence that the changes in the warm core are due to the advection of dry air into the eyewall and the penetration of this air to lower levels from the $23^{\text {rd }}$ to the $24^{\text {th }}$. There is evidence that the wind shear caused additional cooling on the $24^{\text {th }}$ above $10 \mathrm{~km}$, however the cooling is small compared to the cooling due to the dry air entrainment into the eyewall. On the $23^{\text {rd }}$ about $98 \%$ of the hydrostatic pressure drop occurs below $10 \mathrm{~km}$, 
while on the $24^{\text {th }}$ about $92 \%$ of the pressure drop occurs below $10 \mathrm{~km}$. This indicates that only about $6 \%$ of the cooling is due to ventilation of the upper warm core. This is in contrast to the study by Frank and Ritchie (2001) where modeled storms were shown to decrease in intensity under moderate to high shear due to the ventilation of the upper level warm core.

The RHI scans offer additional evidence that the updraft is affected by the entrainment of dry air into the eyewall updraft. On the $23^{\text {rd }}$, in the northern eyewall (Fig. 57d) the highest reflectivity of 40-51 dBZ abruptly stops at $6 \mathrm{~km}$, the level of dry air entrainment on the $23^{\text {rd }}$. On the $24^{\text {th }}$, the highest reflectivity in the eyewall (Fig. 57e) of $37 \mathrm{dBZ}$ stops at $4 \mathrm{~km}$, with a drastic decrease in reflectivity above this level. These observations offer supporting evidence that the eyewall updrafts are losing some of their buoyancy and decreasing the $\theta_{\mathrm{e}}$ at the levels where dry air is entraining into the eyewall.

The cooling of about $3^{\circ} \mathrm{C}$ at $2 \mathrm{~km}$ is due to a rise in the inversion layer. Based on past research, intensifying TCs typically have warm dry eyes above a marked inversion that tends to descend during intensification. During periods of filling, the inversion level tends to ascend and the eye soundings are moister (Jordan 1961; Franklin et al. 1988; Willoughby 1998). The rise in the inversion level from the $23^{\text {rd }}$ to the $24^{\text {th }}$ by $1 \mathrm{~km}$ as Humberto fills matches past observations and causes increased cooling at $2 \mathrm{~km}$ in Fig. 77.

\subsection{The Complexity of Humberto: Contrasts to WISHE and Axisymmetry}

\subsubsection{A Comparison of WISHE with Late Stage Genesis on the $22^{\text {nd }}$}

Bosart and Sanders (1981), Velasco and Fritsch (1987), Menard and Fritsch (1989), Frank and Chen (1991), Chen and Frank (1993), Bister and Emanuel, 1997, Simpson et al. (1997), and Rodgers and Fritsch (2001) have hypothesized that if a MCS 
moves into an area favorable for genesis to occur, the upper level warm core in the stratiform rain region plays a crucial role in tropical cyclogenesis. Bister and Emanuel (1997) suggest the mid-levels under the stratiform rain region moisten and the lower troposphere becomes nearly saturated. The associated MCV is advected to the surface and convection is initiated due to increased surface fluxes with increased wind speeds. The saturated mid and lower troposphere precludes the development of cold, dry downdrafts that would inhibit TC development. A warm core in this scenario would occur in the upper atmosphere, as the fluxes from the sea surface increase and air ascends on warmer moist adiabats compared to the environment.

As the aircraft first sample the storm on the $22^{\text {nd }}$ an arc of convection $50 \mathrm{~km}$ north of the circulation center (Fig. 30) is the dominant feature. The associated MCV is present (Fig. 32); however, this is where the observations on the $22^{\text {nd }}$ and the Bister and Emanuel (1997) theory diverge. The warm core (Fig. 38) first forms in the lower troposphere under the trailing edge of the anvil, due to an area of mesoscale descent. The descending air forms a layer that warms dry adiabatically. The air in the core of the storm is not saturated as suggested by Bister and Emanuel (1997) and the nascent warm core has dew point depressions of $12 \mathrm{~K}$. Inconsistent with WISHE theory, the maximum temperature perturbation is located in the lower troposphere. Over $90 \%$ of the surface pressure fall is due to this warm dry layer. WISHE theory assumes the eye to be a mechanically driven artifact of the storm. In Humberto it seems that the nascent warm core or the "eye" forms first and initiates a series of events that lead to the intensification of the vortex.

Warm values of $\theta_{\mathrm{e}}$ are not associated with increased wind speeds at the surface as assumed by WISHE theory, but are building up under the nascent warm core. The low- 
level warm core is capping the atmosphere, allowing the increase in fluxes. It is not until a dome of high $\theta_{\mathrm{e}}$ of about $360 \mathrm{~K}$ has built up that convection occurs on the weak side of the cap and a nascent eyewall forms.

4.7.2 A Comparison of WISHE with Hurricane Humberto on the $22^{\text {nd }}, 23^{\text {rd }}$, and $24^{\text {th }}$

The idealized, axisymmetric, WISHE model (Emanuel, 1986) is a widely accepted theory for the intensification of TCs. Besides the asymmetric kinematic and thermodynamic structures observed in Humberto, which are in contradistinction to any axisymmetric model, I compare the idealized WISHE model with thermodynamic observations in Humberto.

To effectively obtain the maximum temperature perturbation if WISHE was acting in Humberto, the lowest $\theta_{\mathrm{e}}$ along the transect that the Humberto temperature perturbation was constructed was compared with the highest $\theta_{\mathrm{e}}$ observed by the WP-3D in the eyewall. Taking the lowest temperature profile on the periphery of the transects and the highest saturated $\theta_{\mathrm{e}}$ from observations in the eyewall from the WP-3D is the maximum temperature perturbation based on WISHE. Any warming beyond the WISHE profile is therefore due to subsidence barring any warmer $\theta_{\mathrm{e}}$ found near the eye or eyewall. On the $22^{\text {nd }}$ the WP-3D observed the first convective outbreak of the eyewall, which was the highest $\theta_{\mathrm{e}}$ measured for the entire day. On the $23^{\text {rd }}$ and $24^{\text {th }}$, there were 12 eyewall passes on each day, sampling the entire eyewall annulus. On the $22^{\text {nd }}$, (Fig. 78a) the WISHE temperature perturbation and the Humberto temperature perturbation show large contrasts. The expected warming in the upper troposphere from WISHE does not exist. Most of the warming of the atmosphere is due to subsidence through the layer and not higher $\theta_{\mathrm{e}}$ in the eyewall. 
The temperature perturbation on the $23^{\text {rd }}$ (Fig. 78b) shows increased warming in the lower troposphere and decreased warming above approximately $6 \mathrm{~km}$ when compared to WISHE. The decrease in warming above $6 \mathrm{~km}$ is due to the entrainment of dry air into the eyewall. On the $24^{\text {th }}$, the highest $\theta_{\mathrm{e}}$ from the eyewall (348K) was used. Higher $\theta_{\mathrm{e}}$ (350-352) was observed in the eye above the BL. The red dashed line in Fig. 78c displays the temperature anomaly based on WISHE if the eye $\theta_{\mathrm{e}}(352 \mathrm{~K})$ is used. Even if the higher eye $\theta_{\mathrm{e}}$ is used, there is still a considerable area of warming due to subsidence. These arguments do not discount that increased fluxes from the sea surface with increased winds speeds are not an important component of Humberto's development. Equivalent potential temperatures on the $23^{\text {rd }}$ and $24^{\text {th }}$ increase traveling cyclonically from the upshear quadrant of the storm to the downshear quadrant. The higher $\theta_{\mathrm{e}}$ is warming the troposphere, although subsidence warming and a reduction of $\theta_{\mathrm{e}}$ due to dry air entrainment are also large components of the distribution of warming through the vertical extent of the troposphere. 


\section{CHAPTER 5}

\section{Summary}

\subsection{Conclusions}

Plan views and vertical cross-sections of kinematic and thermodynamic structures constructed from over 200 GPS sondes are utilized to examine the evolution of Humberto over the course of 3 consecutive days. High altitude GPS sondes from NASA's ER-2 and the DC-8 along with GPS sondes from 2 NOAA WP-3Ds allow for an investigation of Humberto through the depth of the troposphere. A thorough investigation of every GPS sonde is made to correct the key variables, especially specific humidity and to omit spurious data. The GPS sonde data are coupled with WP-3D flight level data and radar in an effort to document the salient thermodynamic and kinematic features of Humberto as it intensifies from a tropical storm to a category 2 hurricane and then weakens to a category 1 . The kinematic and thermodynamic structures are compared with theoretical models, idealized models, and past observations as a means to identify similarities or differences with structures present in the models and prior observations. The significant findings are as follows:

1) As the WP-3D first enters Humberto on the $22^{\text {nd }}$, the reflectivity is characterized by an arc of convection $50 \mathrm{~km}$ north northeast of the low-level circulation center. An $\mathrm{MCV}$ is present above $500 \mathrm{hPa}$ and is advecting the exhaust from the convective arc over the circulation center. Beneath the trailing edge of the anvil the nascent warm core forms in the lower troposphere with minimal warming at upper levels. 
2) The warm core on the $22^{\text {nd }}$ forms from a subsiding warm dry layer and not higher $\theta_{\mathrm{e}}$ air moving up the eyewall column. The warm dry layer beneath $5 \mathrm{~km}$ accounts for $90 \%$ of the surface pressure reduction.

3) High BL $\theta_{\mathrm{e}}$ air on the $22^{\text {nd }}$ is not collocated with the highest surface winds as would be expected from WISHE theory. A dome of high $\theta_{\mathrm{e}}$ air from the surface to $2 \mathrm{~km}$ is collocated with low wind speeds and the surface circulation center. An energy budget demonstrates the capping of the BL, the unusual $\theta_{\mathrm{e}}$ profiles with warmer $\theta_{\mathrm{e}}$ near the sea surface, and the divergence in the core of the TS are all vital ingredients in the creation of the high $\theta_{\mathrm{e}}$ dome.

4) High CAPE builds under the nascent warm core on the $22^{\text {nd }}$ due to high convective inhibition. When the instability is released, deep convective clouds form on the downshear side of the warm core, forming the nascent eyewall.

5) As the VWS increases and Humberto becomes a TC, the kinematic fields demonstrate persistent relationships with VWS vector. The highest tangential winds are located to the left of the VWS vector. The radial winds display inflow-outflow couplets aligned with the VWS vector with the strongest convergence located in the downshear quadrant of the storm.

6) Equivalent potential temperature increases to the right of the shear vector and decreases to the left of the shear vector. High CAPE and low CIN are collocated with the downshear quadrant of the eyewall. Low CAPE and High CIN are collocated with the upshear quadrant of the eyewall

7) The warm core throughout the experiment is observed to have its maximum temperature anomaly in the lower troposphere contrary to past observations and 
theory. Unexpectedly, the warm core is observed to build from the lower troposphere to the upper troposphere as Humberto intensifies from a TS to a category 2 TC.

8) A comparison of the warm core anomaly in Humberto with what WISHE predicts yields unexpected results. Humberto has persistent warming in the lower troposphere above that of WISHE with cooling in the upper troposphere. The warming is due to subsidence in the eye and the cooling is due to dry air entrainment in the eyewall.

9) As Humberto weakens from a category 2 to a category 1 storm under high VWS, the warm core in the upper troposphere undergoes negligible changes. The largest cooling of the warm core occurs in the mid-troposphere as the level of dry air entrainment into the eyewall penetrates from 6 to $4 \mathrm{~km}$. This is unlike what is expected from past modeling studies of weakening storms in high VWS where the largest cooling of the warm core is expected in the upper troposphere.

\subsection{Summary and Discussion}

\subsubsection{Formation of Tropical Storm Humberto}

As the WP-3D first enters Humberto on the $22^{\text {nd }}$, when it is a $1000 \mathrm{hPa}$ tropical storm, the reflectivity pattern consists of an arc of convection approximately $50 \mathrm{~km}$ north of the low level circulation center. GPS sonde observations show that an MCV is present with its center about $50 \mathrm{~km}$ north- northeast of the low-level circulation center. RHI scans clearly show an anvil created from the convective arc that is being advected over the low-level circulation center by the mid-level winds associated with the MCV. Under the trailing edge of the anvil and over the low-level circulation center, a subsidence signal is evident from multiple low and high level GPS sondes. The soundings in the vicinity of the low-level circulation center have lapse rates below $5 \mathrm{~km}$ that are approximately dry 
adiabatic with dew point depressions of over $12 \mathrm{~K}$. The maximum temperature perturbation over the low-level circulation center is about $7 \mathrm{~K}$ at $2 \mathrm{~km}$. High altitude GPS sondes record an approximately $1 \mathrm{~K}$ temperature perturbation at $400 \mathrm{hPa}$. The warm dry layer below $5 \mathrm{~km}$ is caused by subsiding air and not higher $\theta_{\mathrm{e}}$ air moving up the eyewall column. A downdraft of 2 to $6 \mathrm{~m} \mathrm{~s}^{-1}$ is present for a $25 \mathrm{~km}$ distance during the passage of the WP-3D at $5 \mathrm{~km}$ on the periphery of the circulation center. Rain rates over the circulation center are a few mm per hour. The model results from Leary (1980) show that with light rain rates ( 1-5 mm per hour) and a pre-existing mesoscale downdraft originating at the base of the anvil, soundings that closely resemble the thermodynamic structures observed by Zipser (1977) are produced. An existing mesoscale downdraft with similar rain rates to Leary (1980) allows the air to continue its descent without becoming saturated and causes a deep layer of warm dry air to form. The pressure reduction at the sea surface is due to this warm dry layer.

The reflectivity, wind and thermodynamic fields of Humberto are asymmetric, like most tropical storms. In contrast to this asymmetry is the warmest equivalent potential temperatures found in the storm's center. This reservoir of warm $\theta_{\mathrm{e}}$ is nearly concentric with respect to the center and is about $2 \mathrm{~km}$ deep and is underneath a strong inversion. The reservoir is created as air southeast of the center spirals inward. The surface fluxes are of the magnitude to account for the increased $\theta_{\mathrm{e}}$. High convective inhibition in the core prevents the high $\theta_{\mathrm{e}}$ air from erupting into cumulonimbi. This creates a situation where CAPE builds markedly, more than an order of magnitude higher than what has been recorded near the eyewall in previous mature hurricanes. As the air either breaks through the lid or escapes laterally an arc of deep convective clouds, $15 \mathrm{~km}$ 
high, develops about $25 \mathrm{~km}$ from the low-level circulation center. This new convection forms the nascent eyewall of Humberto.

The high CAPE has the potential to produce strong accelerations in the vertical that can concentrate the vorticity via the stretching (divergence) term. This may be the most effective avenue for spin-up given that the environment for a developing tropical storm has small vertical shear of the horizontal wind (Gray 1968, McBride and Zehr 1981).

The fields associated with Humberto are similar to that shown by Molinari et al. (2004) and Heymsfield et al. (2006) in that the warmest $\theta_{\mathrm{e}}$ was never collocated with the highest surface winds. This is in contradistinction to a mature TC where the inflow gains energy most rapidly in the high wind annulus under and radially outward of the eyewall.

\subsubsection{Structure of Humberto in a High Vertical Wind Shear Environment}

Humberto develops into a TC as the VWS increases to its highest values on the $23^{\text {rd }}$ and $24^{\text {th }}$. As shown in earlier studies (Bender 1997, Frank 1998, Black et al. 2002, Eastin et al. 2005, Frank and Ritchie 2001), the eyewall convection develops a wave one asymmetry under the high shear conditions on the $23^{\text {rd }}$ and $24^{\text {th }}$. Eyewall convection is concentrated in the downshear left quadrant of the storm. The kinematic and thermodynamic fields show persistent relationships to the VWS vector. The highest tangential winds are concentrated left of the shear vector, while the radial winds show strong inflow-outflow couplets aligned with the VWS vector. The strongest convergence in the BL is located in the downshear quadrants of the storm. The distribution of $\theta_{\mathrm{e}}$ in the BL near the eyewall increases to the right of the shear vector and decreases to the left of the shear vector. The higher $\theta_{\mathrm{e}}$, present in the downshear 
quadrant, is collocated with higher CAPE and low to no CIN. Lower $\theta_{\mathrm{e}}$, lower CAPE, and high CIN are located in the upshear quadrant and effectively suppress convection in the eyewall on the upshear side of the storm. These observations support past research by Black et al. (2002) and Eastin et al. (2005) that a mesoscale ascent maximum was located downshear in the eyewall with a minimum in the upshear quadrants.

\subsubsection{Thermodynamic Structures and the Intensity of Humberto}

The vertical cross-sections of $\theta_{\mathrm{e}}$ and temperature exhibit large contrasts compared to past views by Hawkins and Rubsam (1968), Hawkins and Imbembo (1976), and Jorgensen (1984). From a simple axisymmetric view of intensification, the average column $\theta_{\mathrm{e}}$ in the eyewall would be expected to correlate with the intensity of the storm. As Humberto transitioned from a TS to a Category 2, $\theta_{\mathrm{e}}$ evolved from warm values concentrated in the BL to an asymmetric distribution with warmer values in the downshear eyewall above the BL. The lower troposphere has increased warming in the upshear eyewall column, compensating for the lower $\theta_{\mathrm{e}}$ collocated with this quadrant. On the $24^{\text {th }}$ after a period of weakening, the $\theta_{\mathrm{e}}$ distribution above the BL had increased values in the eye compared to the eyewall, similar to observations by Kossin and Eastin (2001). Eye to eyewall mixing was observed on the $22^{\text {nd }}$ and $23^{\text {rd }}$, demonstrating that the low-level eye continued to surrender energy to the eyewall during the experiment.

Observing the evolution of the warm core, an unexpected result is realized. The warm core is observed to build from the lower troposphere to the upper troposphere as the storm intensifies. The maximum temperature anomaly is in the lower troposphere on all 3 days. The comparison of the Humberto temperature anomalies with that expected by WISHE showed large contrasts. The maximum warming based on WISHE, which is 
that due to parcels with warmer $\theta_{\mathrm{e}}$ ascending along warmer pseudo-adiabats, should occur in the upper troposphere. Any additional warming beyond the WISHE profile is due to subsidence. Humberto's temperature anomalies have persistent warming beyond that of the WISHE profile in the lower troposphere and cooling in the upper troposphere. The difference in the cooling from the $23^{\text {rd }}$ to the $24^{\text {th }}$ showed that the maximum cooling was present in the mid-troposphere. This is contrary to past modeling studies (Frank and Ritchie 2001) that display cooling in the upper troposphere under moderate to high VWS due to the ventilation of the warm core. The cooling in Humberto under high VWS is attributed to the entrainment of dry air into the eyewall updraft and eye and the penetration of this dry air from 6 to $4 \mathrm{~km}$ from the $23^{\text {rd }}$ to the $24^{\text {th }}$.

The warming in the lower troposphere beyond that of the WISHE profile needs further investigation. However, the subsidence warming shows similarities to what might be expected if the idealized models of Shapiro and Willoughby (1982) and Holland and Merrill (1984) are taken into consideration. In their models a heat source placed in the inertially stable eyewall can have two effects; one is to increase the secondary circulation of the TC, another is to cause dry adiabatic descent on the inner edge of the eyewall and produce an associated hydrostatic pressure fall in the eye. Radial flows in the northern eyewall on the $23^{\text {rd }}$ show an outflow-inflow couplet in the northern eyewall and outflow throughout the lower eye above the BL which would cause subsiding air in the eye (see appendix Fig. 2). The entrainment of dry environmental air with low $\theta_{\mathrm{e}}$ into the eyewall would effectively decrease the magnitude of the heat source. The lowering of the subsidence warming in Humberto from $6 \mathrm{~km}$ on the $23^{\text {rd }}$ to about $4-5 \mathrm{~km}$ on the $24^{\text {th }}$ is 
likely related to the entrainment of dry air and the a decrease in the magnitude of the heat source.

\subsubsection{Overall View of the Evolution of Humberto}

In the initial stages Humberto develops in moderate to low shear, over warm SSTs, and a vorticity rich environment. The only inhibiting factor is the dry environmental air. Plan views and vertical cross-sections of the relative humidity on the $22^{\text {nd }}$, show that the environment within $1^{\circ}$ of the core has high relative humidity compared to the environment values calculated from the SHIPS model (see appendix Fig. 3). This would suggest that the MCS and the associated MCV have had the effect of preconditioning the core of Humberto by moistening the troposphere. Therefore, it seems that on the $22^{\text {nd }}$, Humberto is in ideal conditions for formation and intensification. It is quite possible that the observations on the $22^{\text {nd }}$ of the formation of the warm core are between early formation, where a warm core has not formed yet, and that of a fully developed TC.

As Humberto evolves into a TC on the $23^{\text {rd }}$ and $24^{\text {th }}$, increased surface fluxes with increased wind speeds are occurring as suggested by Kleinschmidt (1951), Malkus and Riehl (1960), and Emanuel's (1986) WISHE theory, however they are occurring in the context of an asymmetric system. The unusual warm core structures on the $23^{\text {rd }}$ and $24^{\text {th }}$ may apply to systems that evolve in dry environments under high shear. Further investigation is needed to determine whether the structures observed in Humberto are a more general pathway to formation and intensification or if these structures exist solely because of the high latitude, increasing wind shear, and the dry environment. 


\subsection{Future Work}

In the future, it would be advantageous to investigate more systems with the methods used in this dissertation. In general systems in the depression and tropical storm stage have not been thoroughly investigated with a combination of GPS sondes, radar, and aircraft data. Many storms in the early stages of development have been sampled with GPS sondes, but detailed analysis of these storms is still sparse. An investigation of storms that are of lower intensity in different environmental conditions would serve to verify whether Humberto is an anomaly or a more general pathway to formation. If TCs at lower categories display maximum temperature anomalies in the lower troposphere, similar to Humberto, then the observations that the warm core builds from the lower troposphere to the upper troposphere would be verified and possibly apply to all TCs. This work would help to increase the accuracy of model intensity forecasts.

The plethora of data during the Humberto experiment is unprecedented. The addition of environmental soundings would allow for numerous modeling studies to test the structures calculated in the models with the structures in Humberto. Asymmetries in the thermodynamic and kinematic fields along with the unusual warm core structures offer precise observations that can be compared with model structures. This would allow the physical parameters in the model to be adjusted so that more accurate forecasts could be made in the future. Although much work is certainly needed to increase the accuracy of intensity forecasts, Humberto offers a chance to model a TC as it intensifies and weakens over 3 days. A number of idealized modeling studies could also be useful to understand more thoroughly the physical processes occurring at different stages of TC evolution. These would include: the transformation of a tropical wave into a warm cored 
system, the development of asymmetric systems, the effect of VWS and dry

environmental air on a TC, and the evolution of the maximum temperature anomaly from the lower troposphere to the upper troposphere as a TS intensifies to into a mature TC. 


\begin{tabular}{lcccc}
\hline & $\begin{array}{c}\text { Operating } \\
\text { range }\end{array}$ & Accuracy & Resolution & $\begin{array}{c}\text { Time } \\
\text { constant }\end{array}$ \\
\hline Pressure & $20-1060 \mathrm{mb}$ & $0.5 \mathrm{mb}$ & $0.1 \mathrm{mb}$ & $<0.01 \mathrm{~s}$ \\
Temperature & $-90^{\circ}$ to $40^{\circ} \mathrm{C}$ & $0.2^{\circ} \mathrm{C}$ & $0.1^{\circ} \mathrm{C}$ & $2.5 \mathrm{~s}$ at $20^{\circ} \mathrm{C}$ \\
& & & & $3.7 \mathrm{~s}$ at $-40^{\circ} \mathrm{C}$ \\
Humidity & $0 \%-100 \%$ & $2 \%$ & $0.1 \%$ & $0.1 \mathrm{~s}$ at $20^{\circ} \mathrm{C}$ \\
& & & & $10 \mathrm{~s}$ at $-40^{\circ} \mathrm{C}$ \\
Wind & $0-150 \mathrm{~m} \mathrm{~s}^{-1}$ & $0.5 \mathrm{~m} \mathrm{~s}^{-1}$ & $0.1 \mathrm{~m} \mathrm{~s}^{-1}$ & \\
\hline
\end{tabular}

Table 1. Manufacturer's specifications for the GPS Dropwindsonde sensors. Accuracy refers to the standard deviation of two successive calibrations (Hock and Franklin 1999). 


\begin{tabular}{|c|c|c|c|c|}
\hline Parameter & Instrument & Range & Resolution & Accuracy \\
\hline $\begin{array}{l}\text { Total air } \\
\text { temperature }\end{array}$ & Rosemont-platinum resistance & $\begin{array}{l}-60 \text { to } \\
+60^{\circ} \mathrm{C}\end{array}$ & $0.03^{\circ} \mathrm{C}$ & $\pm 0.2^{\circ} \mathrm{C}$ \\
\hline Dew point & $\begin{array}{l}\text { General Eastern cooled } \\
\text { mirror }\end{array}$ & $\begin{array}{l}-59 \text { to } \\
+100^{\circ} \mathrm{C}\end{array}$ & $0.03^{\circ} \mathrm{C}$ & $\begin{array}{l} \pm 0.4^{\circ} \text { (dew point) } \\
\pm 0.6^{\circ} \text { (frost point) }\end{array}$ \\
\hline Altitude & APN-59 radar & $\begin{array}{l}60 \mathrm{~m} \text { to } 15 \\
\mathrm{~km}\end{array}$ & & $\pm 0.01 \%$ \\
\hline Ground speed & $\begin{array}{l}\text { Northrop/Delco inertial } \\
\text { navigation with omega } \\
\text { update }\end{array}$ & $0-400 \mathrm{~m} \mathrm{~s}^{-1}$ & $0.06 \mathrm{~m} \mathrm{~s}^{-1}$ & $\pm 0.5 \mathrm{~m} \mathrm{~s}^{-1}$ \\
\hline Track angle & $\begin{array}{l}\text { Northrop/Delco inertial } \\
\text { navigation with omega } \\
\text { update }\end{array}$ & $\pm 180^{\circ}$ & $0.005^{\circ}$ & $\pm 0.2^{\circ}$ \\
\hline Heading & $\begin{array}{l}\text { Northrop/Delco inertial } \\
\text { navigation with omega } \\
\text { update }\end{array}$ & $\pm 180^{\circ}$ & $0.005^{\circ}$ & $\pm 0.1^{\circ}$ \\
\hline Pitch & $\begin{array}{l}\text { Northrop/Delco inertial } \\
\text { navigation with omega } \\
\text { update }\end{array}$ & $\pm 180^{\circ}$ & $0.005^{\circ}$ & $\pm 0.06^{\circ}$ \\
\hline Roll & $\begin{array}{l}\text { Northrop/Delco inertial } \\
\text { navigation with omega } \\
\text { update }\end{array}$ & $\pm 180^{\circ}$ & $0.005^{\circ}$ & $\pm 0.06^{\circ}$ \\
\hline Sideslip angle & Rosemont pressure transducer & -12 to $+12^{\circ}$ & $0.05 \mathrm{kPa}$ & $\pm 0.15 \%$ \\
\hline Attack angle & Rosemont pressure transducer & -12 to $+12^{\circ}$ & $0.05 \mathrm{kPa}$ & $\pm 0.15 \%$ \\
\hline True air spced & $\begin{array}{l}\text { Derived from static and } \\
\text { dynamic pressures and } \\
\text { virtual temperature }\end{array}$ & - & $0.1 \mathrm{~m} \mathrm{~s}^{-1}$ & $\pm 0.5 \mathrm{~m} \mathrm{~s}^{-1}$ \\
\hline $\begin{array}{l}\text { Horizontal } \\
\text { winds }\end{array}$ & $\begin{array}{l}\text { Computed from true air } \\
\text { speed, heading, sideslip } \\
\text { angle, ground speed, and } \\
\text { track }\end{array}$ & $\pm 212 \mathrm{~m} \mathrm{~s}^{-1}$ & $0.1 \cdot \mathrm{m} \mathrm{s}^{-1}$ & $\pm 1.0 \mathrm{~m} \mathrm{~s}^{-1}$ \\
\hline $\begin{array}{l}\text { Vertical } \\
\text { aircraft } \\
\text { acceleration }\end{array}$ & $\begin{array}{l}\text { Northrop/Delco inertial } \\
\text { navigation with omega } \\
\text { update }\end{array}$ & $\pm 20 \mathrm{~m} \mathrm{~s}^{-2}$ & $0.01 \mathrm{~m} \mathrm{~s}^{-2}$ & $\pm 1.0 \mathrm{~m} \mathrm{~s}^{-2}$ \\
\hline $\begin{array}{l}\text { Vertical wind } \\
\text { speed }\end{array}$ & $\begin{array}{l}\text { Computed from integrated } \\
\text { vertical accelerations and } \\
\text { angle of attack, sideslip, } \\
\text { pitch, and roll }\end{array}$ & $\pm 20 \mathrm{~m} \mathrm{~s}^{-1}$ & $0.01 \mathrm{~m} \mathrm{~s}^{-1}$ & $\pm 0.5 \mathrm{~m} \mathrm{~s}^{-1}$ \\
\hline $\begin{array}{l}\text { Aircraft } \\
\text { position }\end{array}$ & $\begin{array}{l}\text { Northrop/Delco inertial } \\
\text { navigation with omega } \\
\text { update }\end{array}$ & & & \\
\hline $\begin{array}{l}\text { Latitude } \\
\text { Longitude }\end{array}$ & & $\begin{array}{l} \pm 90^{\circ} \\
\pm 180^{\circ}\end{array}$ & $\begin{array}{l}8.3 \times 10^{-8} \mathrm{deg} \\
8.3 \times 10^{-7} \mathrm{deg}\end{array}$ & $\begin{array}{l} \pm 1.5 \mathrm{~km} \\
\pm 1.5 \mathrm{~km}\end{array}$ \\
\hline $\begin{array}{l}\text { Dynamic } \\
\text { pressure }\end{array}$ & Garrett transducer & $0-40.6 \mathrm{kPa}$ & $0.04 \mathrm{kPa}$ & $\pm 0.01 \pm 0.00125 \Delta P \mathrm{kPa}$ \\
\hline Static pressure & Garrett transducer & $0-122.0 \mathrm{kPa}$ & $0.04 \mathrm{kPa}$ & $0.02 \mathrm{kPa}$ \\
\hline $\begin{array}{l}\text { Cloud water } \\
\text { content }\end{array}$ & Johnson-Williams hot-wire & $0-6 \mathrm{~g} \mathrm{~m}^{-3}$ & $0.1 \mathrm{~g} \mathrm{~m}^{-3}$ & $\pm 0.20 \%$ \\
\hline
\end{tabular}

Table 2. Instrumentation characteristics of NOAA WP-3D aircraft (Jorgensen 1984a). 


\begin{tabular}{llll}
\hline \multicolumn{1}{c}{ Parameter } & \multicolumn{1}{c}{ Nose radar } & Lower fuselage radar & \multicolumn{1}{c}{ Tail radar } \\
\hline Transmitter frequency & $5445 \pm 6.6 \mathrm{MHz}$ & $5370 \pm 0.7 \mathrm{MHz}$ & $9319 \pm 11.6 \mathrm{MHz}$ \\
Transmitter wavelength & $5.51 \mathrm{~cm}$ & $5.59 \mathrm{~cm}$ & $3.22 \mathrm{~cm}$ \\
Transmitter pulse & $3.0 \mu \mathrm{s}$ & $6.0 \mu \mathrm{s}$ & $0.5 \mu \mathrm{s}$ \\
PRF & $400 \mathrm{PPS}$ & $200 \mathrm{PPS}$ & $1600 \mathrm{PPS}$ \\
Peak transmitter power & $70 \mathrm{~kW}(\mathrm{~min})$ & $70 \mathrm{~kW}(\mathrm{~min})$ & $60 \mathrm{~kW}(\mathrm{~min})$ \\
Receiver dynamic range & $80 \mathrm{~dB}$ & $80 \mathrm{~dB}$ & $80 \mathrm{~dB}$ \\
Gain, main beam & $34 \mathrm{~dB}$ & $37.5 \mathrm{~dB}$ & $40 \mathrm{~dB}$ \\
Gain, sidelobe & $23 \mathrm{~dB}$ down & $23 \mathrm{~dB}$ down & $23 \mathrm{~dB}$ down \\
Horizontal beam width & $3.6^{\circ}$ & $1.1^{\circ}$ & $1.35^{\circ}$ \\
Vertical beam width & $3.6^{\circ}$ & $4.1^{\circ}$ & $1.9^{\circ}$ \\
Antenna stabilization & $\pm 20^{\circ}$ (pitch and roll) & $\pm 10^{\circ}$ (pitch and roll) & $\pm 25^{\circ}$ (pitch and drift) \\
Maximum range & $185 \mathrm{~km}$ & $371 \mathrm{~km}$ & $93 \mathrm{~km}$ \\
\hline
\end{tabular}

Table 3. Airborne radar parameters for NOAA WP-3D aircraft (Jorgensen 1984a). 


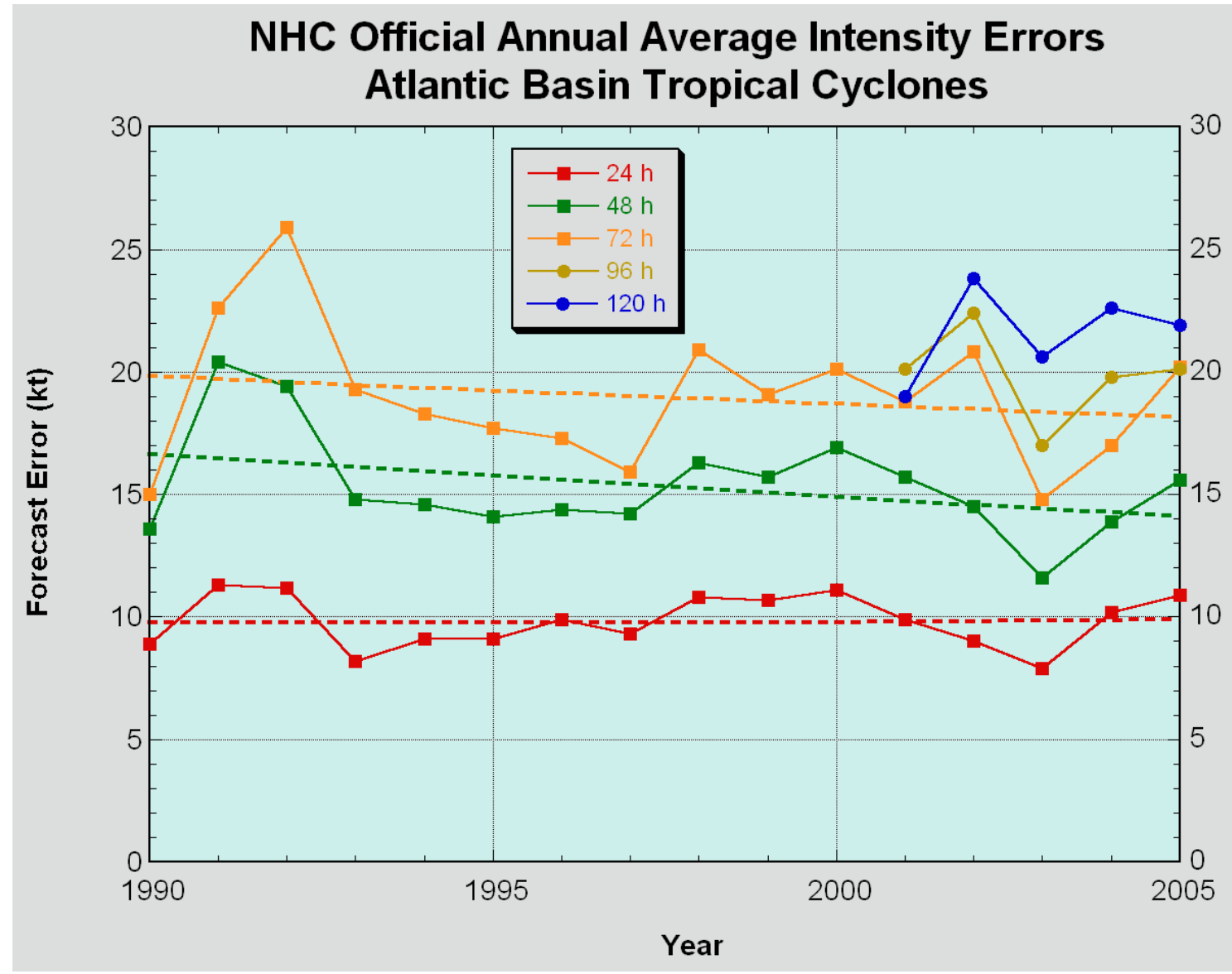

Figure 1. Annual average official intensity errors for Atlantic basin tropical cyclones for the period 1990-2005, with least-squares trend lines superimposed (National Hurricane Center). 


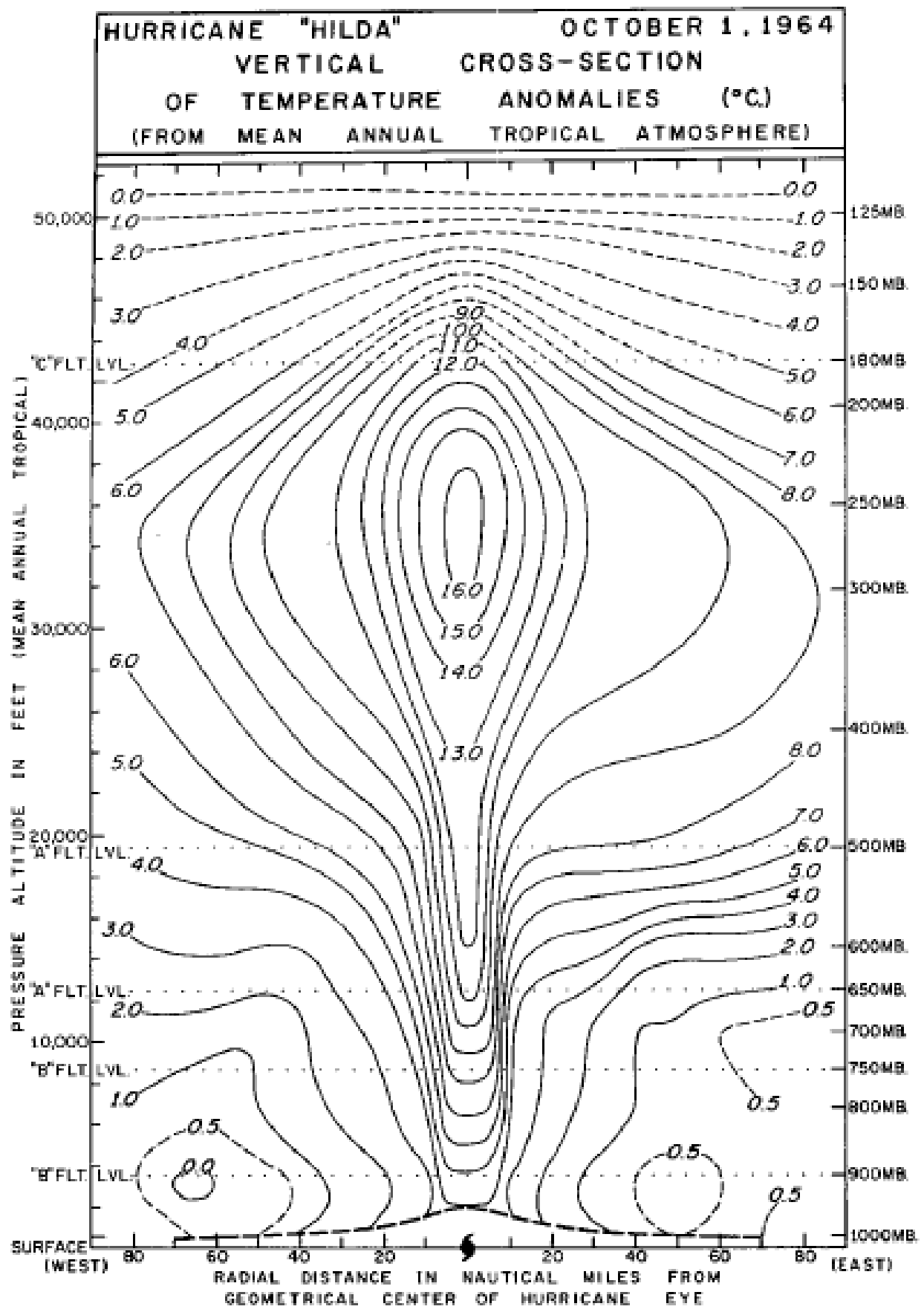

Figure 2. Vertical cross section of the temperature anomaly in Hurricane Hilda prepared from the aircraft and soundings (Hawkins and Rubsam 1968). 


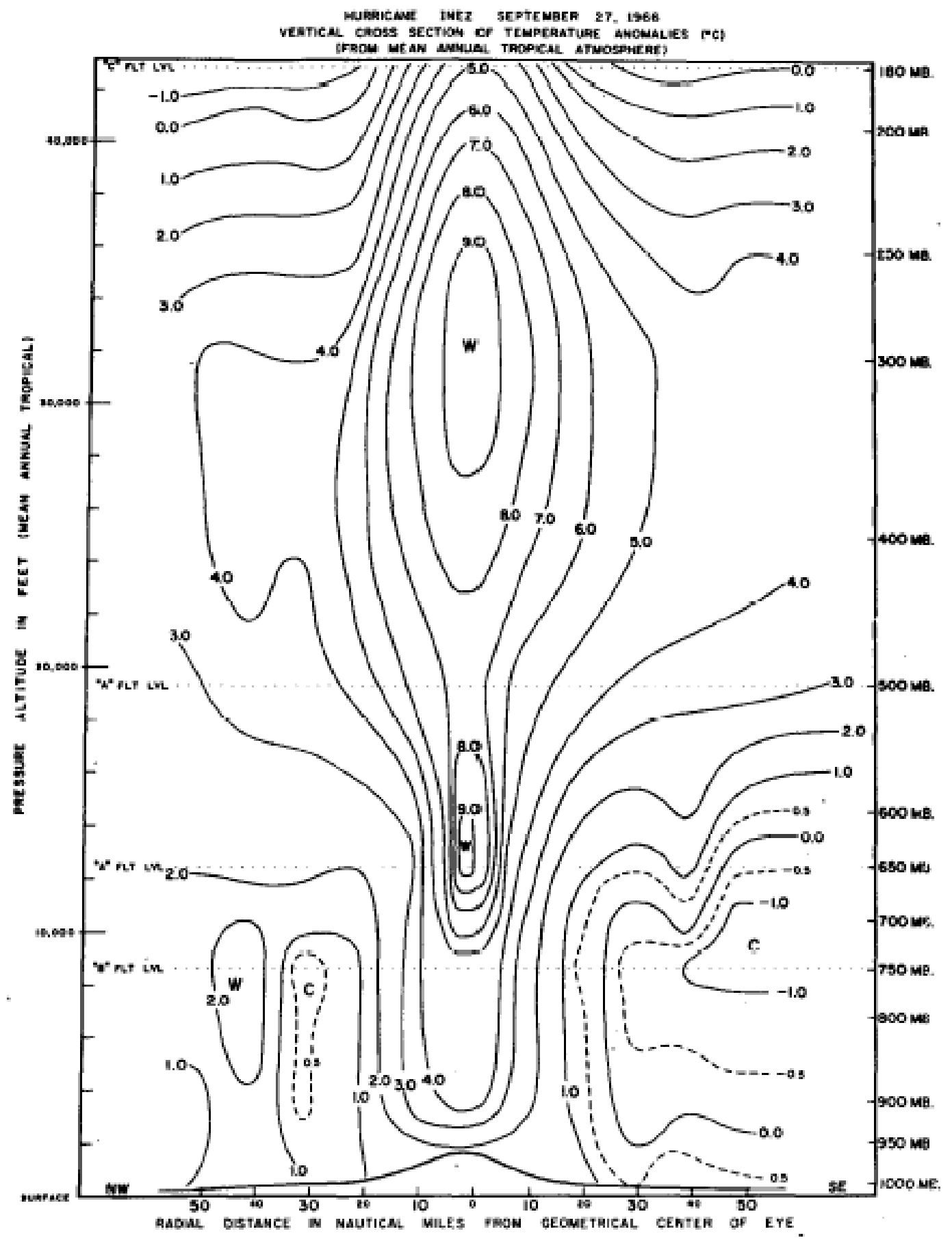

Figure 3. Vertical cross section of the temperature anomaly in Hurricane Inez prepared from the aircraft and soundings. Centers of anomalous warmth, located near the 600 and $300 \mathrm{mb}$ levels (Hawkins and Imbembo 1976). 


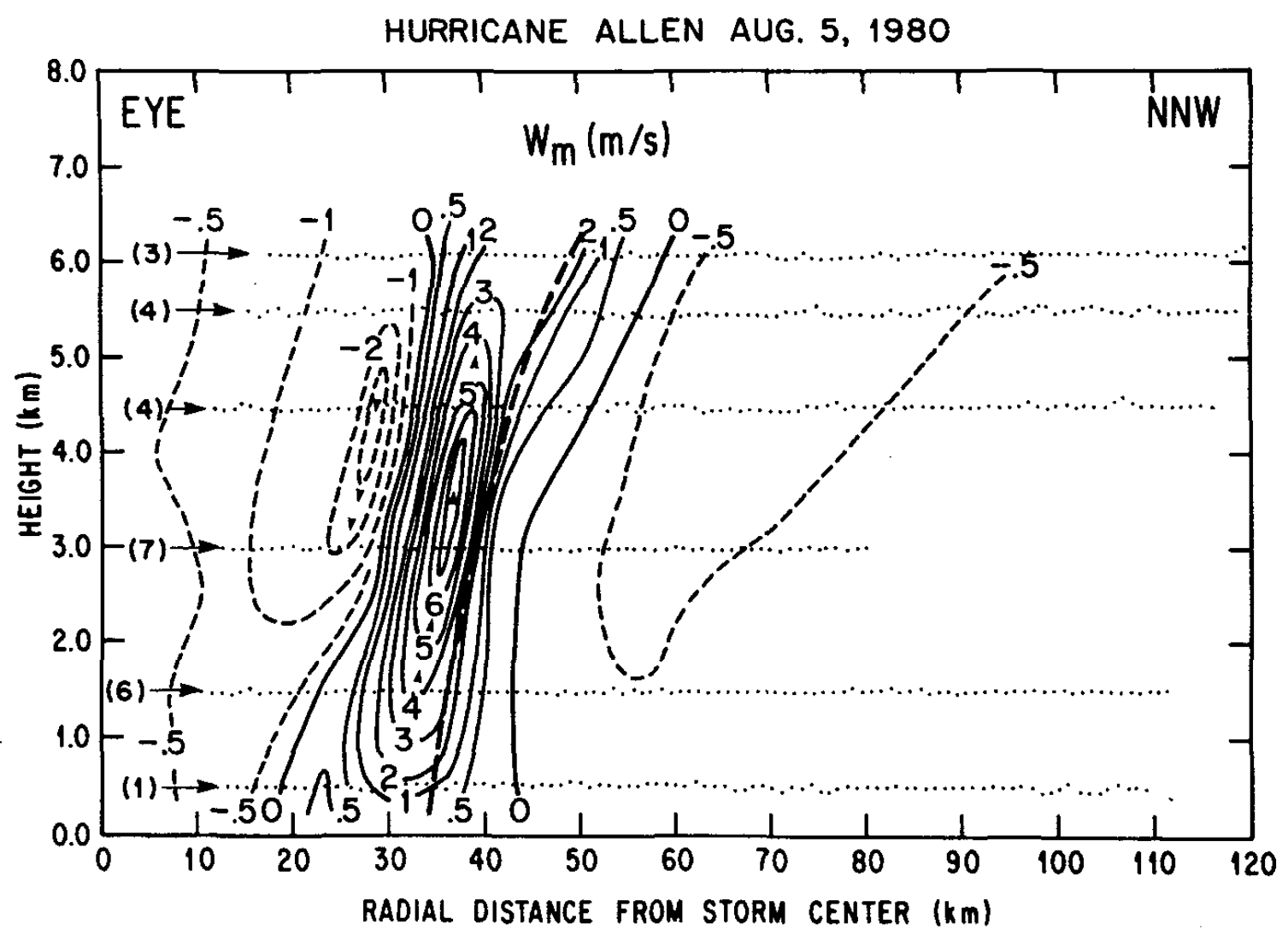

Figure 4. Composite cross section of mesoscale vertical velocity (w) computed by integrating the two-dimensional continuity equation and using the composite radial divergence analysis on 5 August 1980. Dark dashed line is radius of maximum wind (Jorgensen 1984b). 


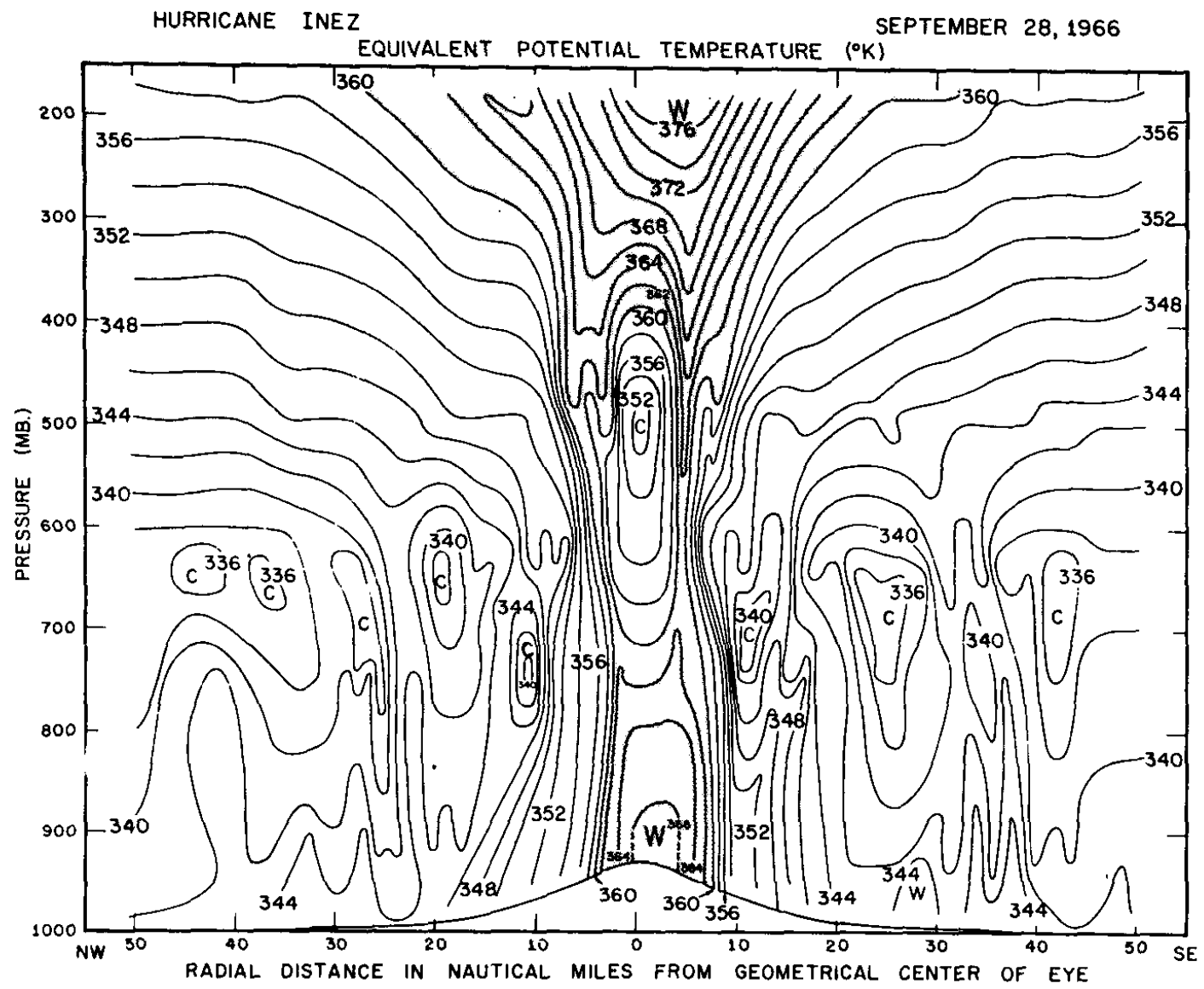

Figure 5. Vertical cross-section of equivalent potential temperature in Hurricane Inez prepared from the aircraft and soundings (Hawkins and Imbembo 1976). 


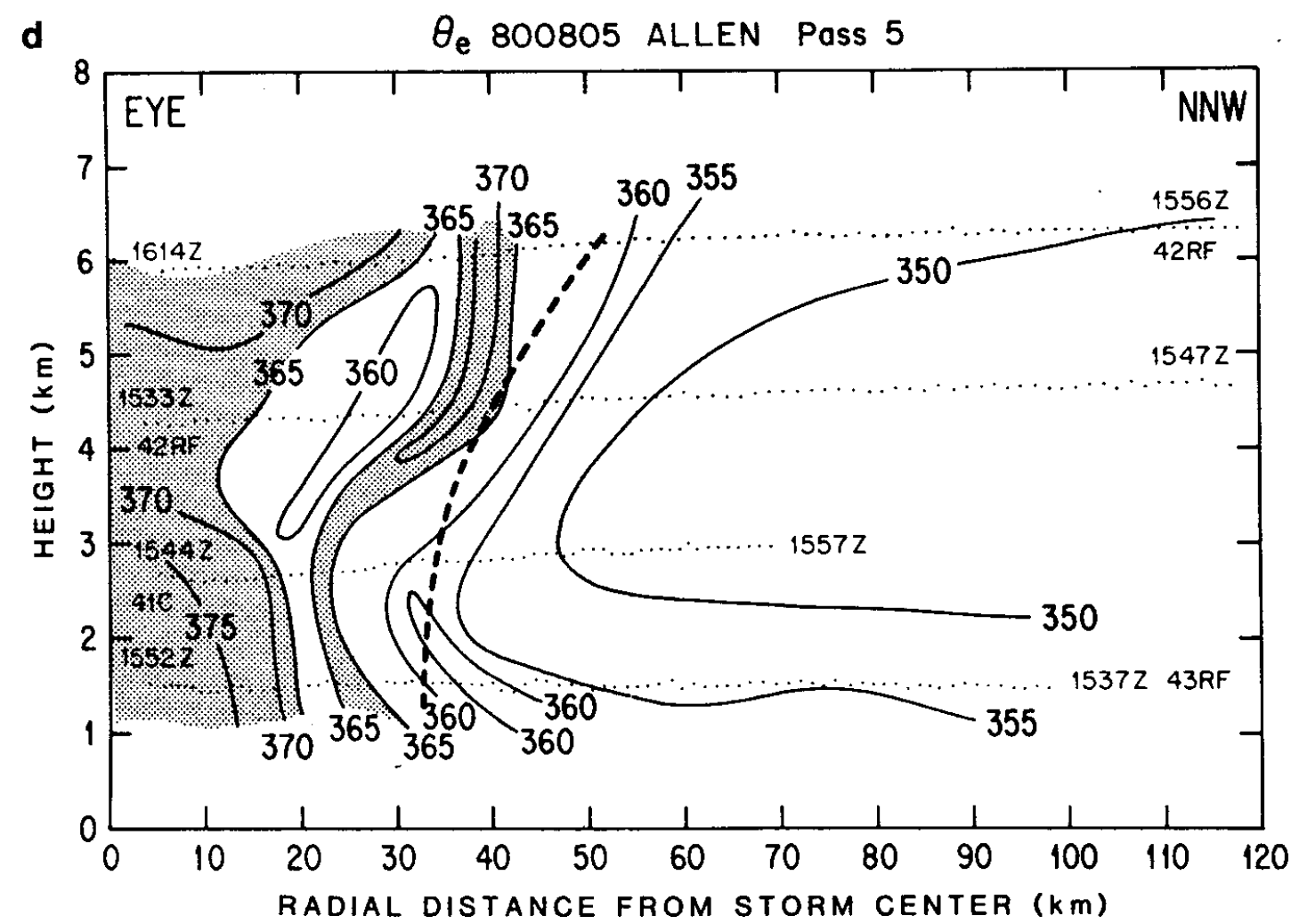

Figure 6. Radius-height cross-section for equivalent potential temperature $\theta_{\mathrm{e}}(\mathrm{K})$. Regions where $\theta_{\mathrm{e}}>365$ are shaded. Heavy dashed line is RMW (Jorgensen 1984b). 


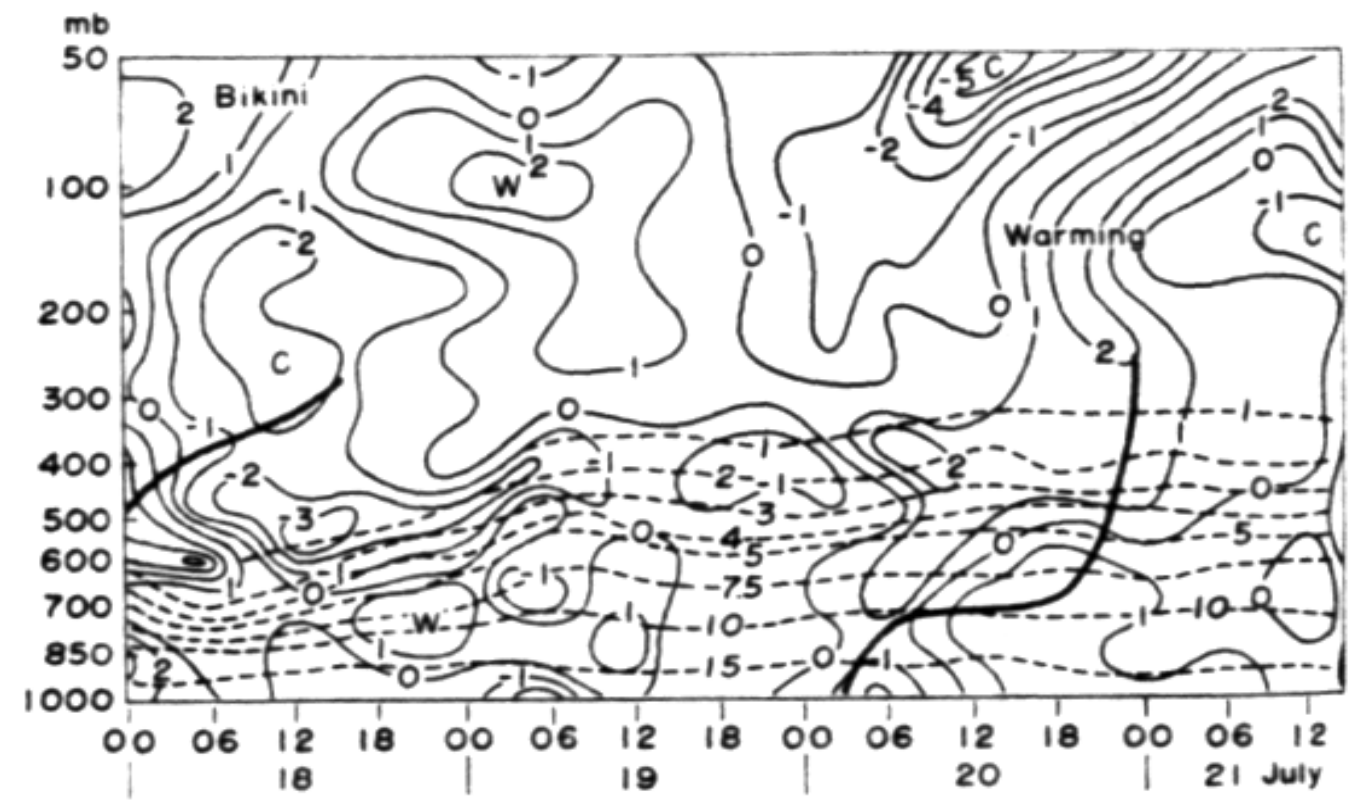

Figure 7. Vertical time section of the 24 hour changes of upper air temperature $\left({ }^{\circ} \mathrm{C}\right)$ at Bikini. Dotted lines show mixing ratios of water vapor $\left(\mathrm{g} \mathrm{kg}^{-1}\right)$ (Yanai 1961).

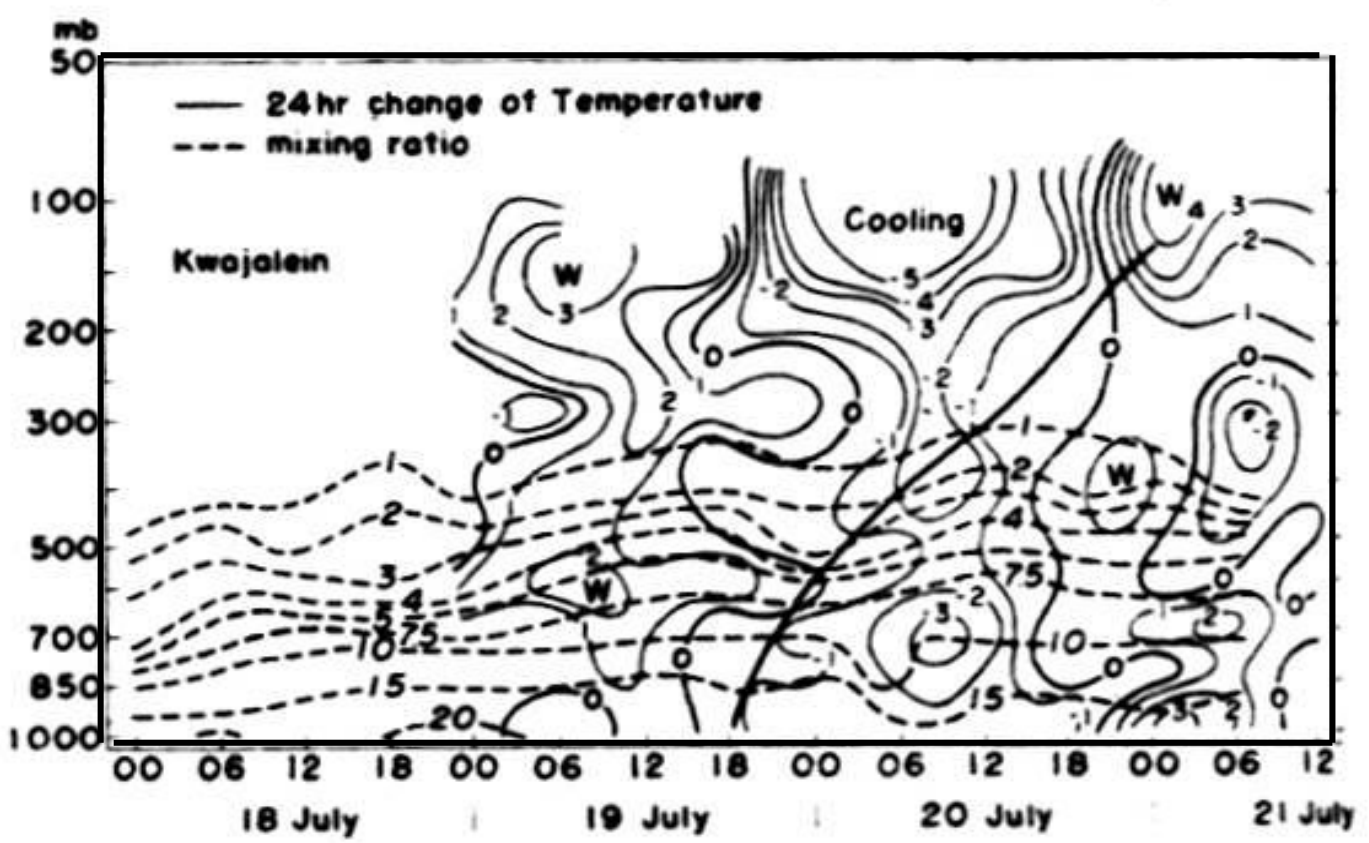

Figure 8. Vertical time section of the 24 hour changes of upper air temperature $\left({ }^{\circ} \mathrm{C}\right)$ at Kwajalein. Dotted lines show mixing ratios of water vapor $\left(\mathrm{g} \mathrm{kg}^{-1}\right)$ (Yanai 1961). 


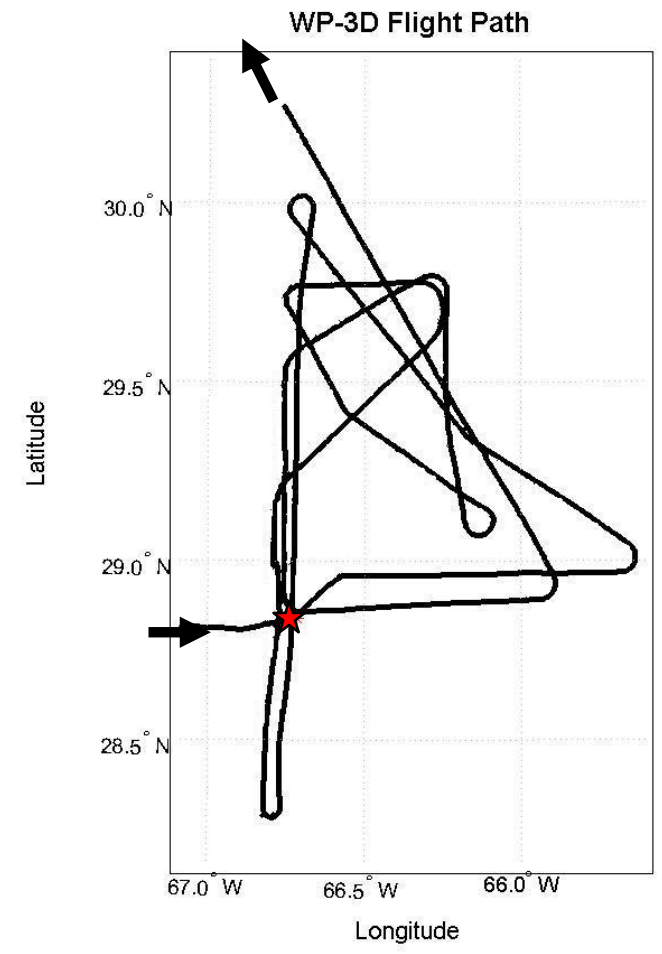

A

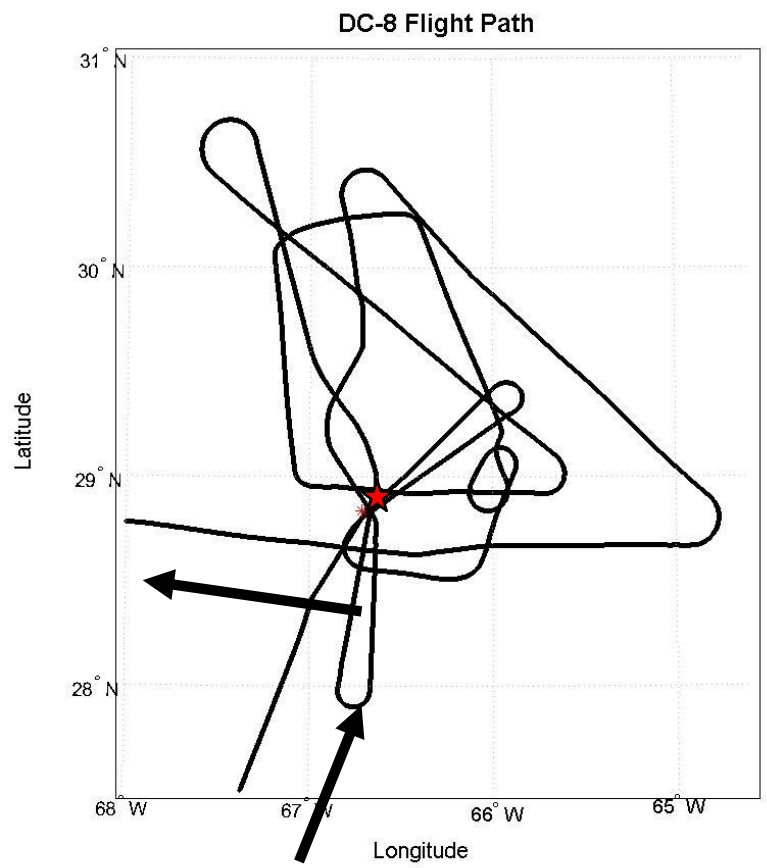

$\mathrm{B}$

Figure 9. (A) Flight track the WP-3D on September $22^{\text {nd }} 2001$. (B) Same as "A" except for the DC-8. Red star shows location of low-level circulation center. Arrows indicate direction of the aircraft. 


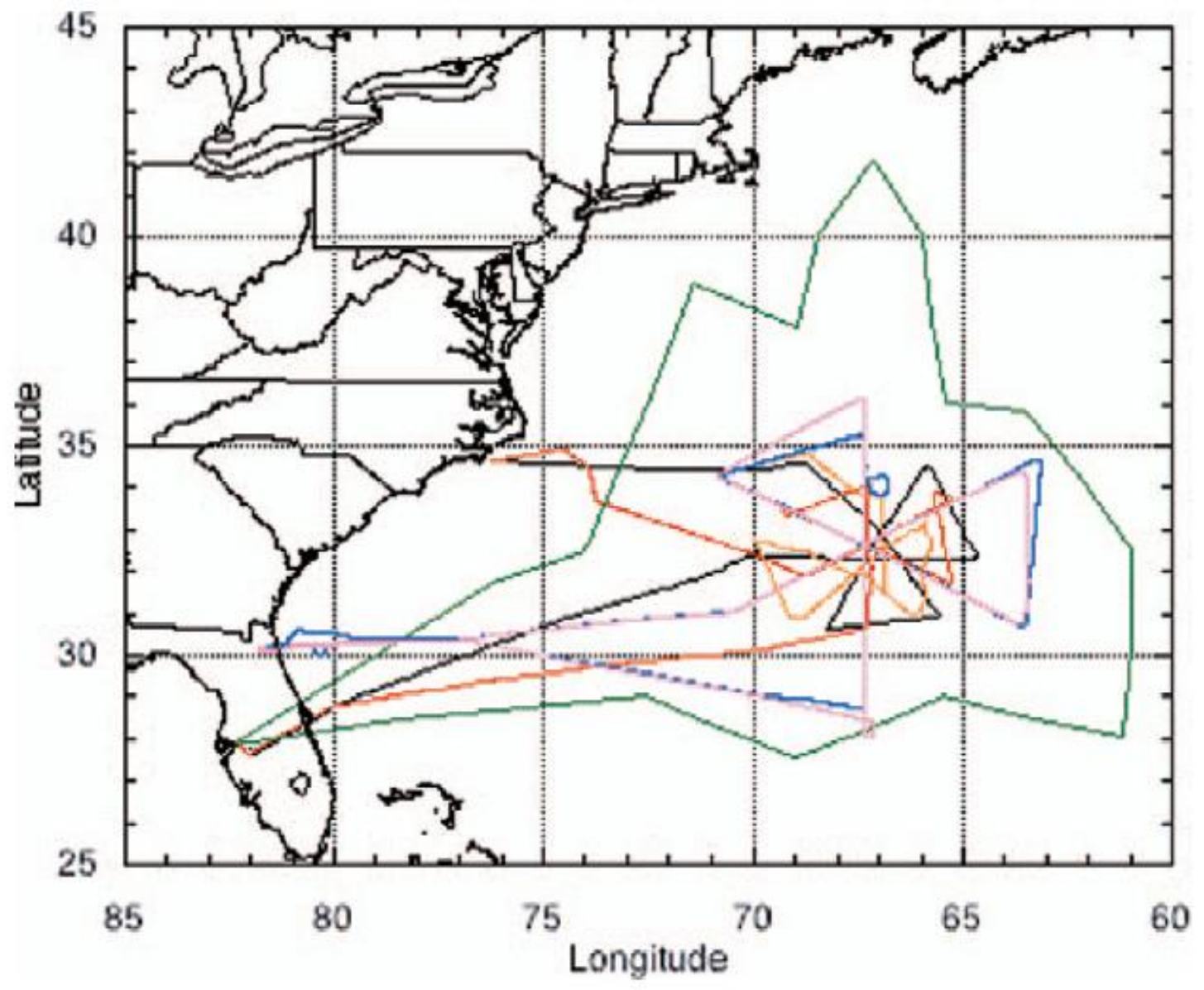

Figure 10. Flight tracks of the six aircraft observing Hurricane Humberto and its stratospheric, tropospheric, and upper-oceanic environment 23-24 September 2001. The red and black lines are the two NOAA WP-3Ds; the blue line is the NASA DC-8; the violet line is the NASA ER-2; and the green line is the NOAA G-IV (Aberson et al. 2006). 


\section{DC-8 and ER-2 Dropwindsone Positions}

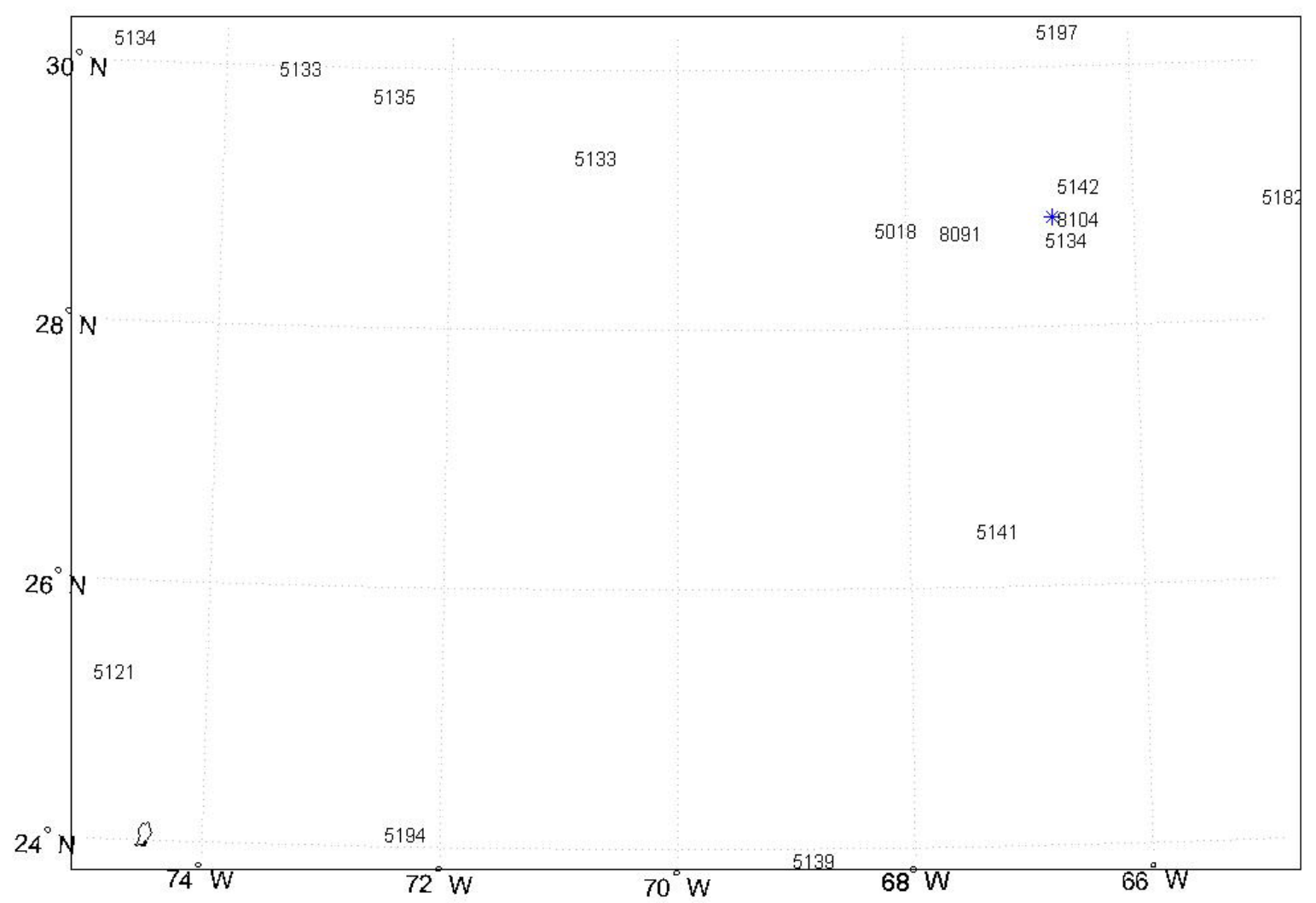

Figure 11. Location of NASA DC-8 and ER-2 dropwindsondes on September $22^{\text {nd }}$. The four digits represent the last four digits of the dropwindsonde number and the space to the left of the four digit number is the location of the jettisoned dropwindsonde. Blue star indicates storm circulation center. 


\section{Position of WP-3d Dropwindsondes}

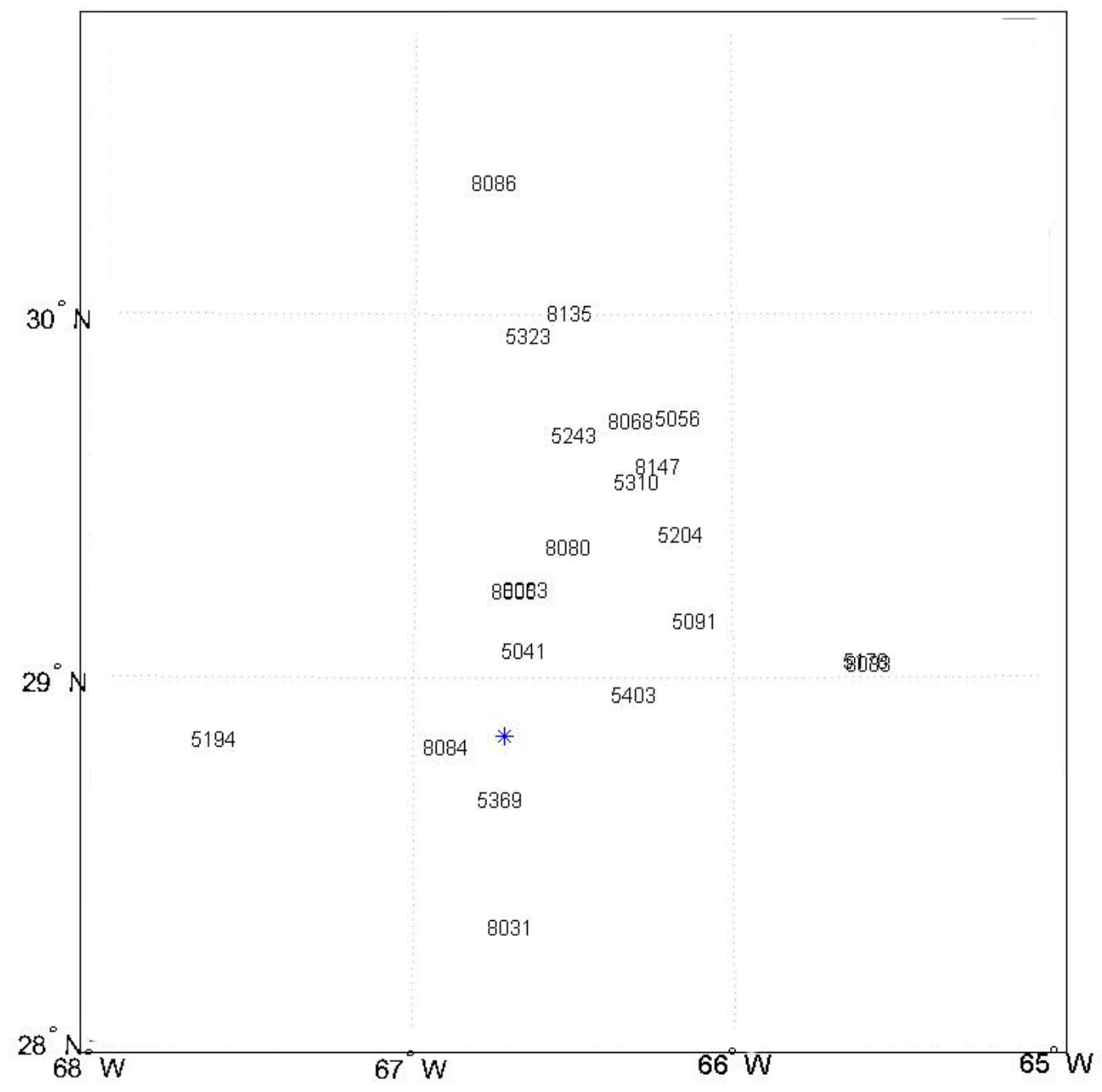

Figure 12. Location of WP-3D dropwindsondes on September $22^{\text {nd }}$. The four digits represent the last four digits of the dropwindsonde number and the space to the left of the four digit number is the location of the jettisoned dropwindsonde. Blue star indicates storm circulation center. 


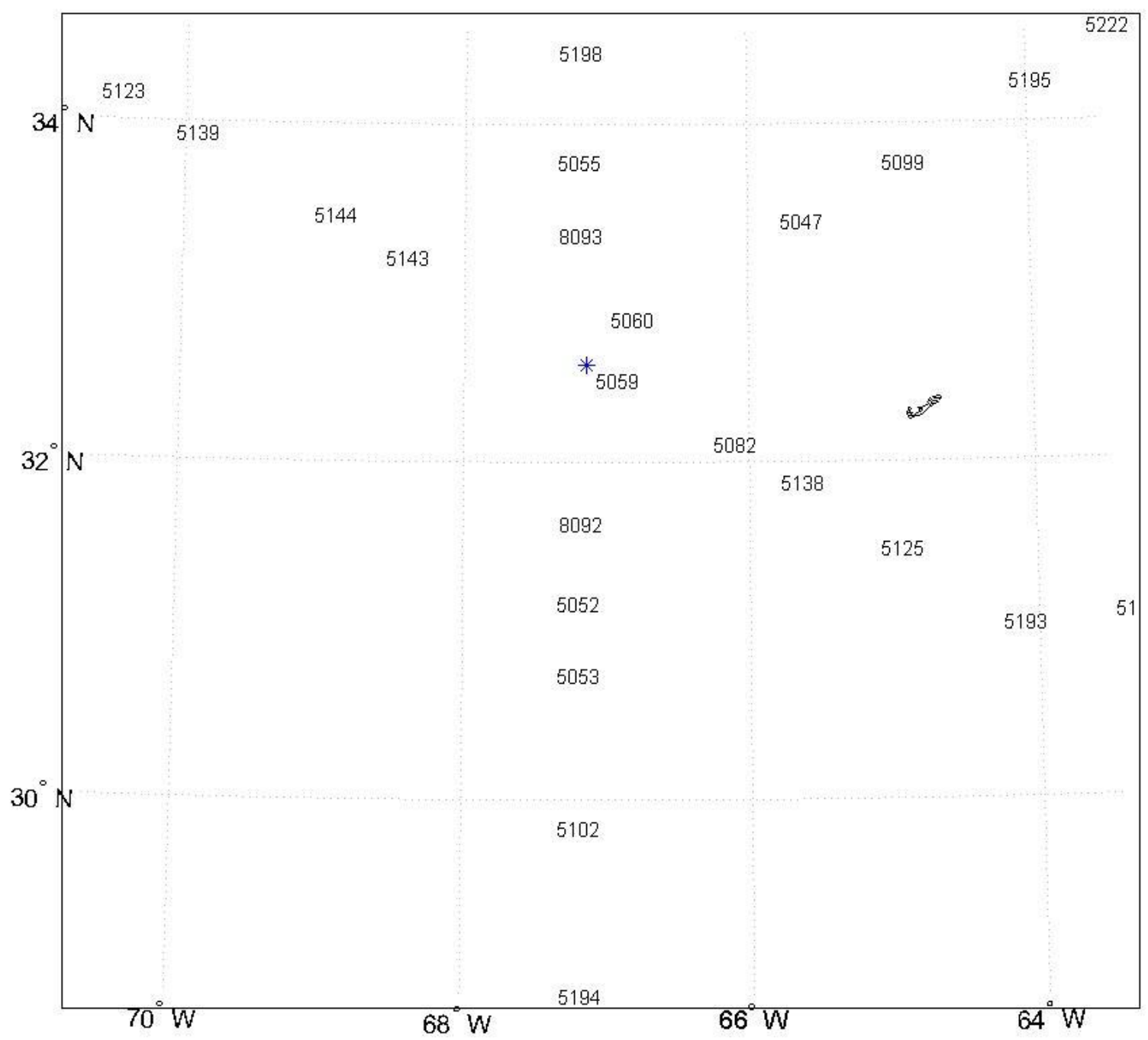

Figure 13. Location of NASA DC-8 and ER-2 dropwindsondes on September $23^{\text {rd }}$. The four digits represent the last four digits of the dropwindsonde number and the space to the left of the four digit number is the location of the jettisoned dropwindsonde. Blue star indicates storm circulation center. 


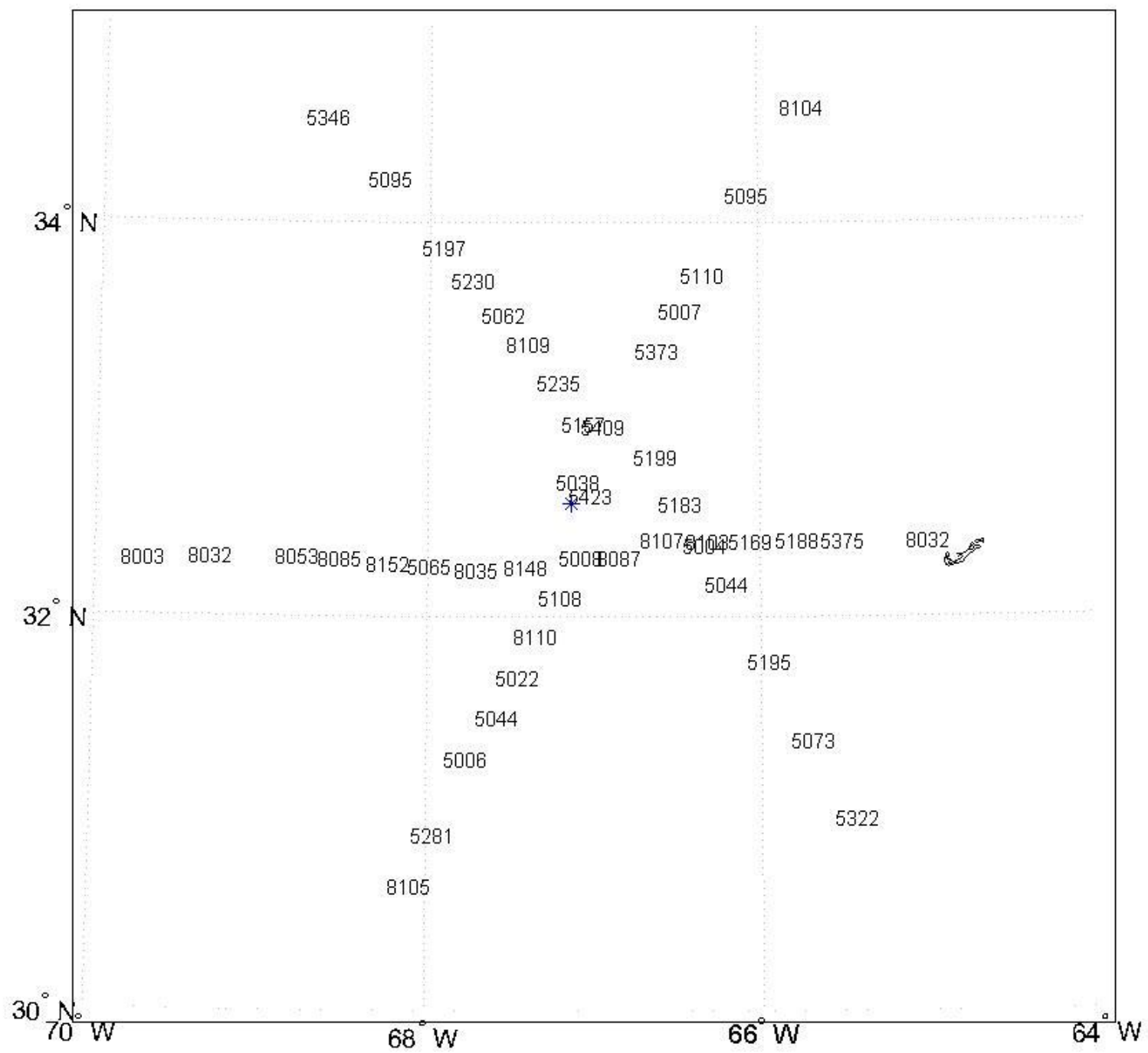

Figure 14. Location of WP-3D (4 km flight level) dropwindsondes on September $23^{\text {rd }}$. The four digits represent the last four digits of the dropwindsonde number and the space to the left of the four digit number is the location of the jettisoned dropwindsonde. Blue star indicates storm circulation center. 


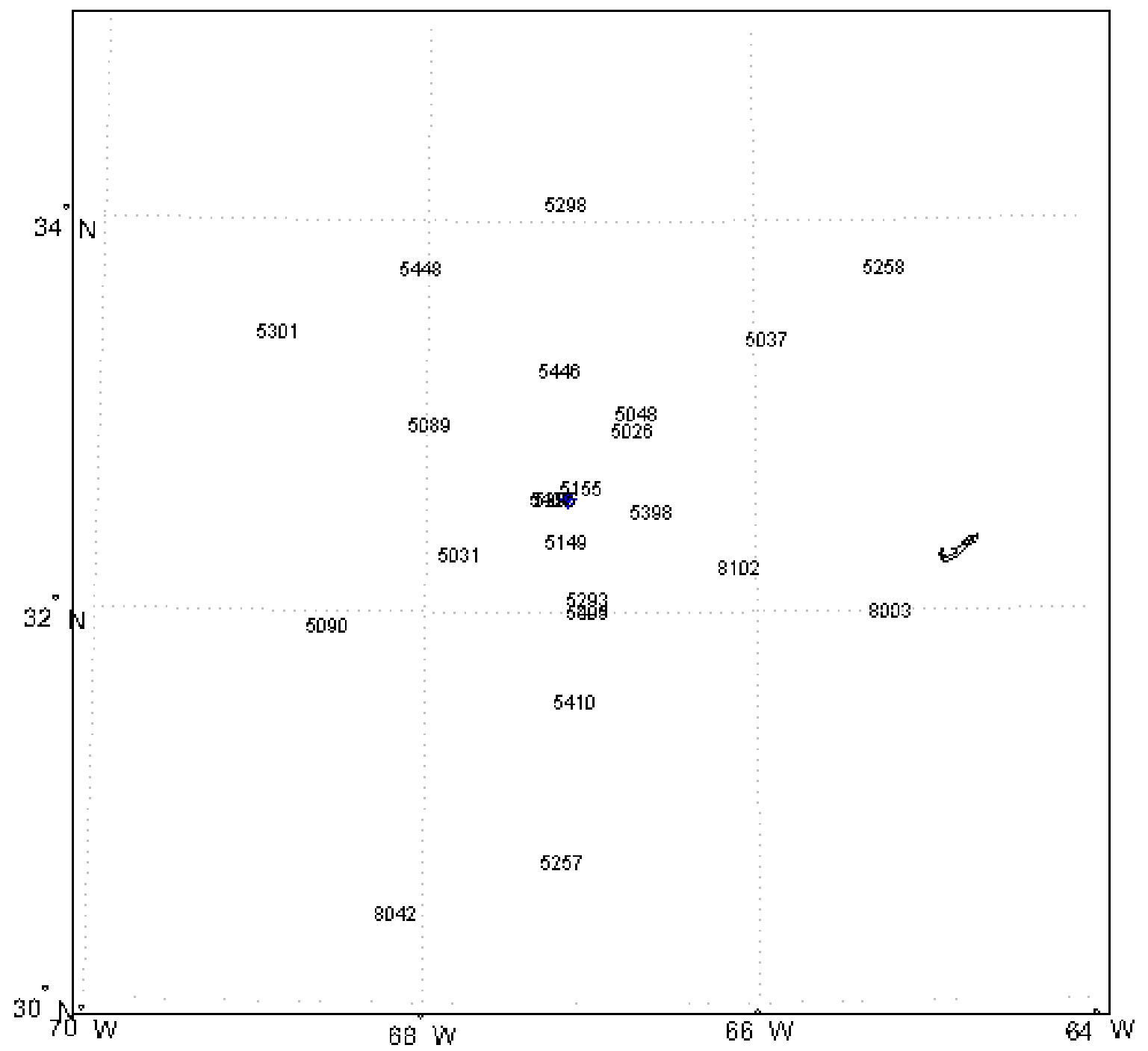

Figure 15. Location of WP-3D (1.5 km flight level) dropwindsondes on September $23^{\text {rd }}$. The four digits represent the last four digits of the dropwindsonde number and the space to the left of the four digit number is the location of the jettisoned dropwindsonde. Blue star indicates storm circulation center. 


\section{NCAR GPS Dropsonde the definitive atmospheric profiling tool}

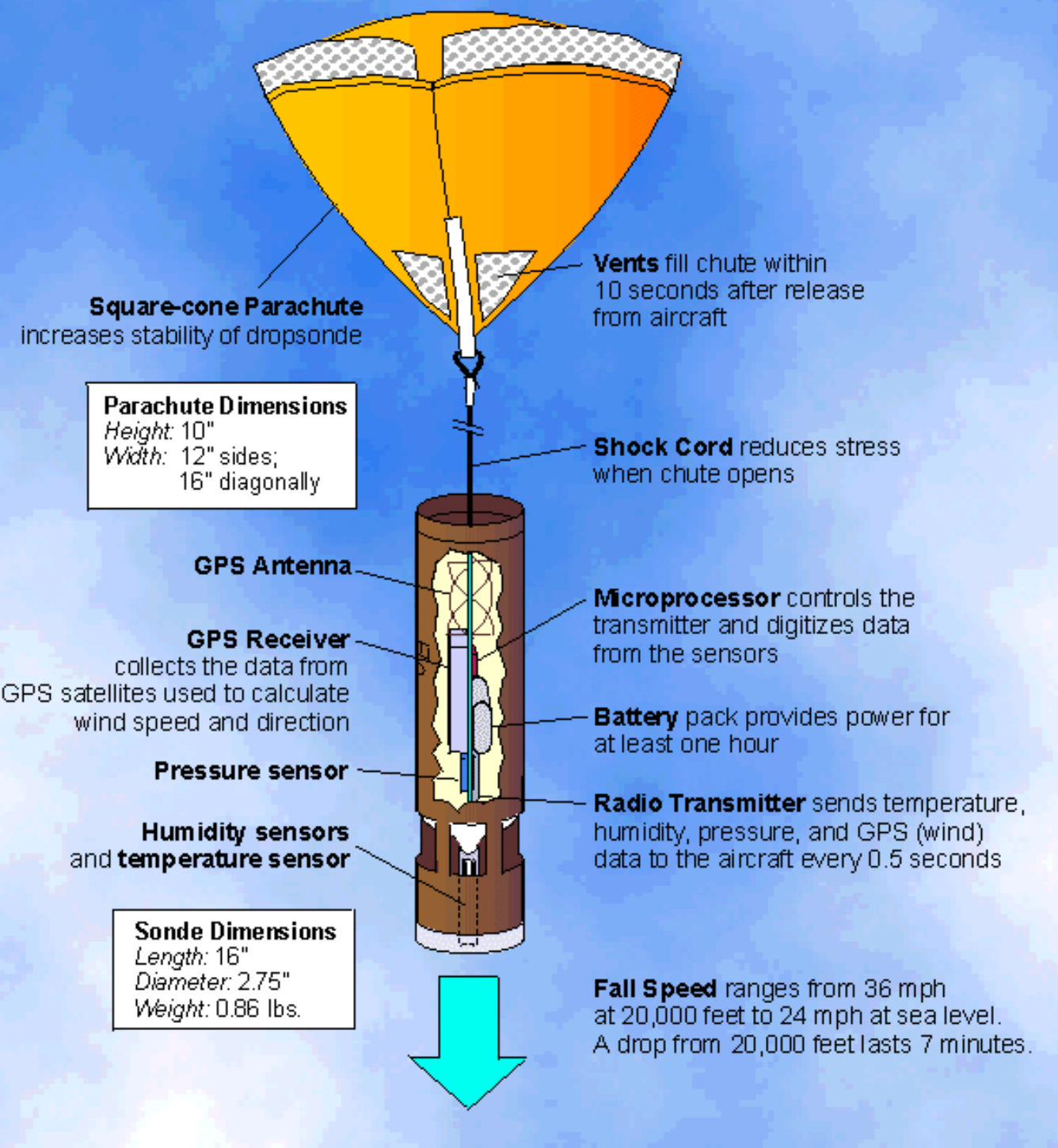

Figure 16. GPS dropwindsonde and internal view (www.eol.ucar.edu ). 


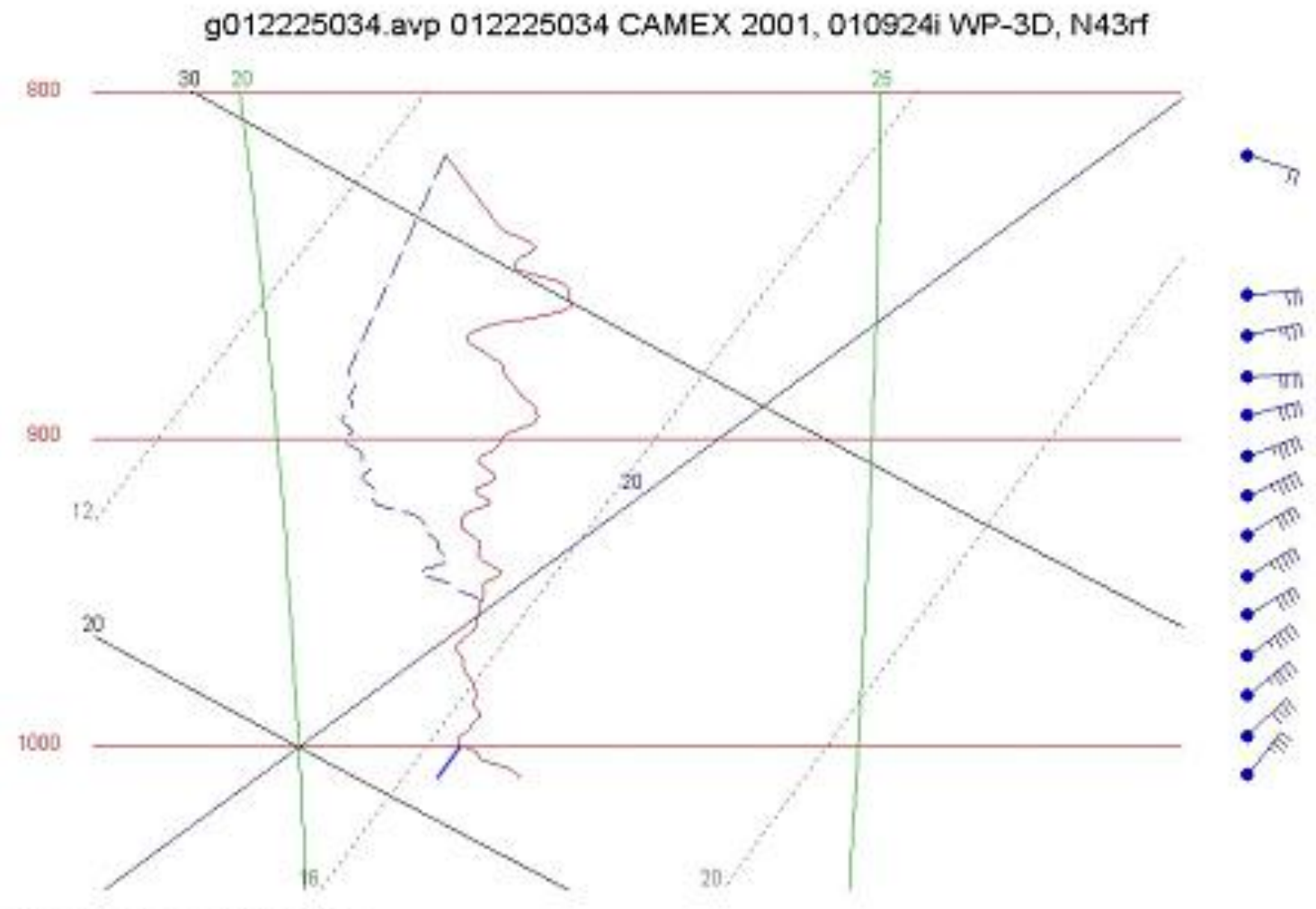

Aspen $v 243.02$ Mir 2004 ox 51 UTC

Figure 17. Skew-T log-P diagram of sensor wetting correction. Red horizontal lines are pressure, blue lines slanted from bottom left to top right indicate temperature, dark blue lines slanted in opposite direction are dry adiabats, curved green lines are moist adiabats, blue dashed lines are water vapor, red line is sounding temperature, and blue dashed line is sounding dew point. Solid blue line shows where the dew point has been corrected. 


\section{g003338085.avp 003338085 Hurricane 2001, 010923H WP-3D, N42RF}

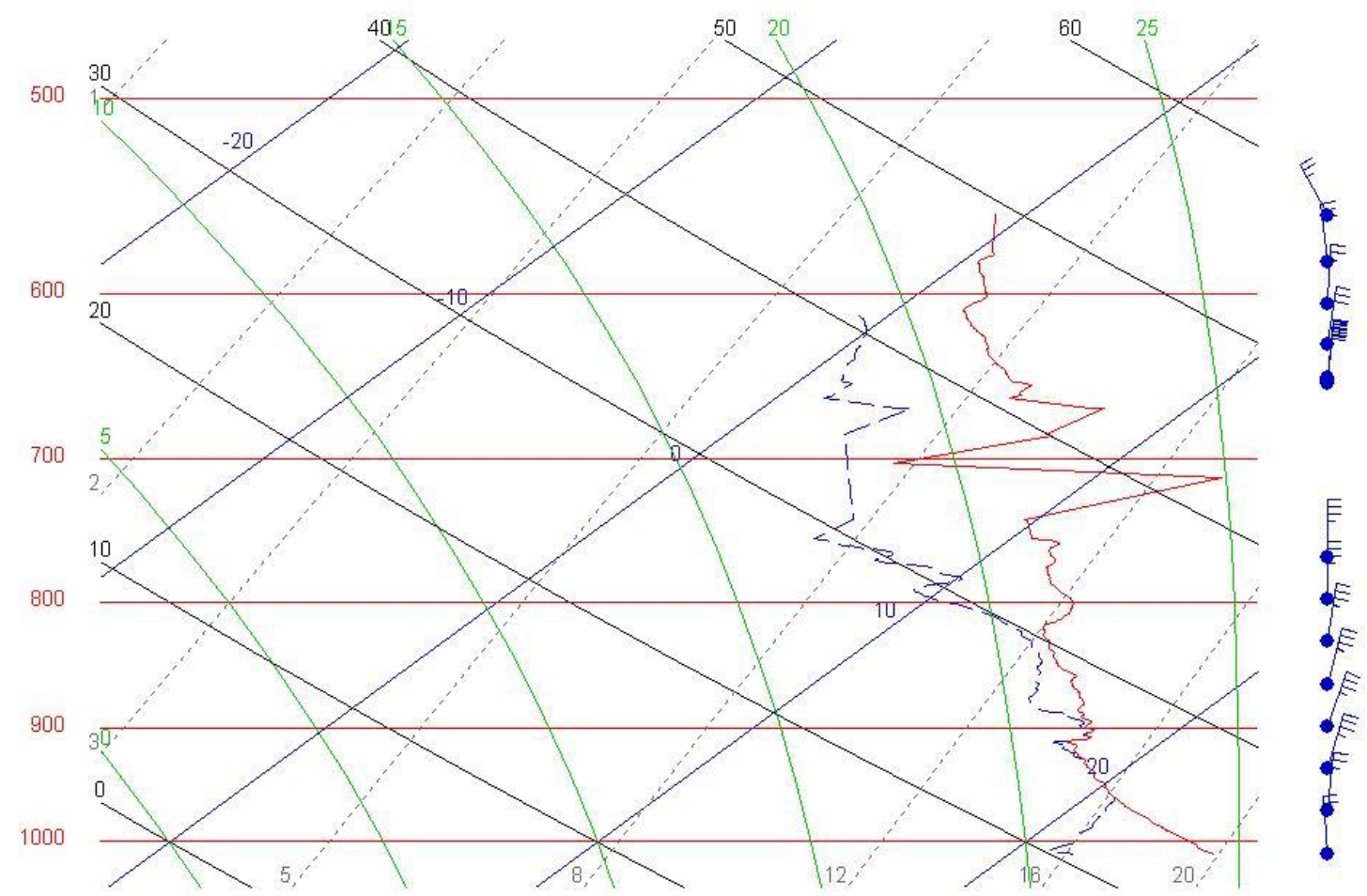

Aspen V2.4.3, 27 Feb 2004 02:14 UTC

Figure 18. Same as Fig. 17 except Skew-T log-P showing radio frequency interference. Area from $660 \mathrm{hPa}$ to $750 \mathrm{hPa}$ was removed. 


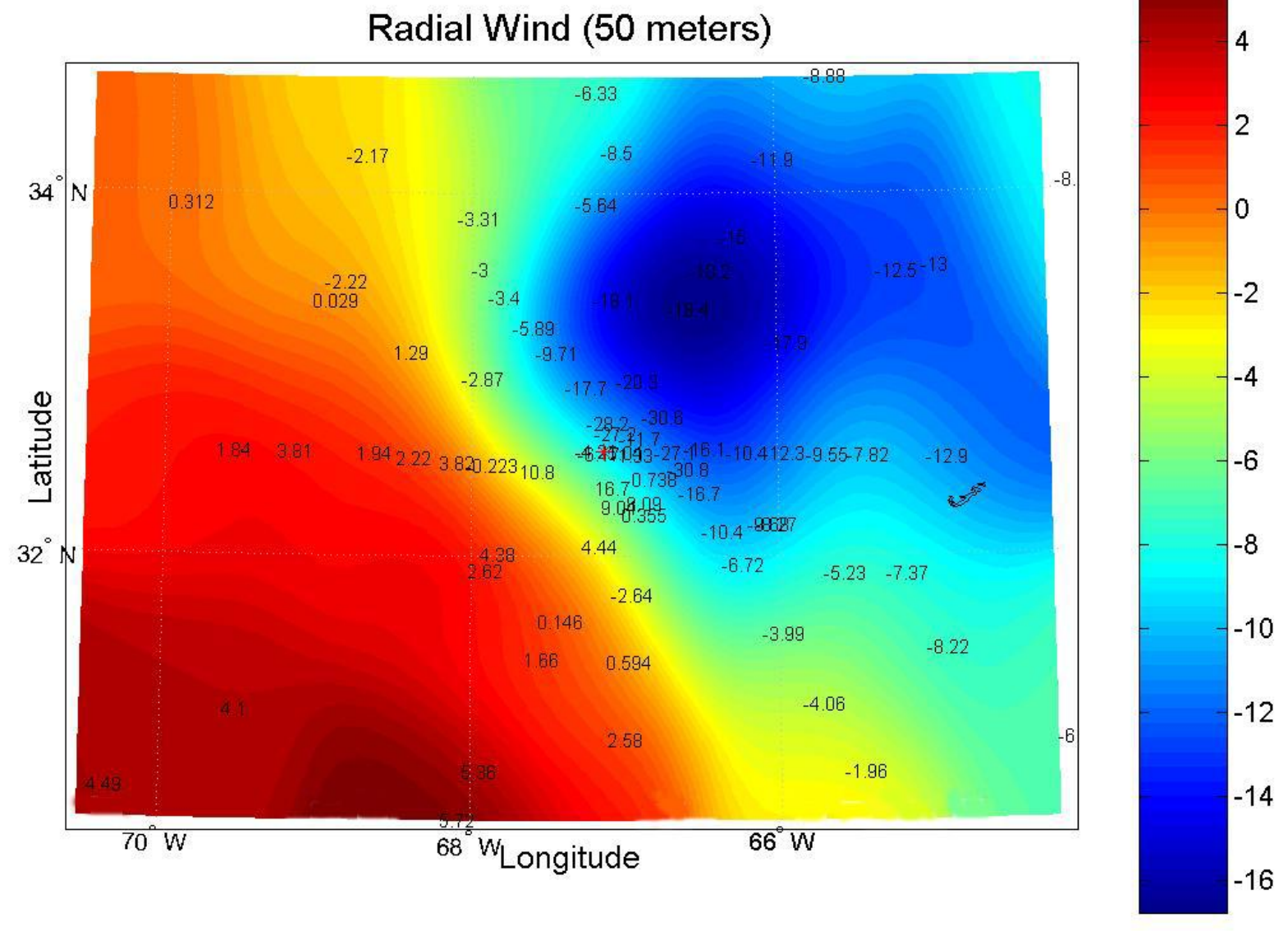

Figure 19. Plan view of the radial winds $\left(\mathrm{m} \mathrm{s}^{-1}\right)$ on the $23^{\text {rd }}$ of September, 2001, using the Barnes Objective Analysis. Numbers represent the exact values that each GPS sonde read. Color bar to the right denotes values given by the Barnes Objective Analysis. 

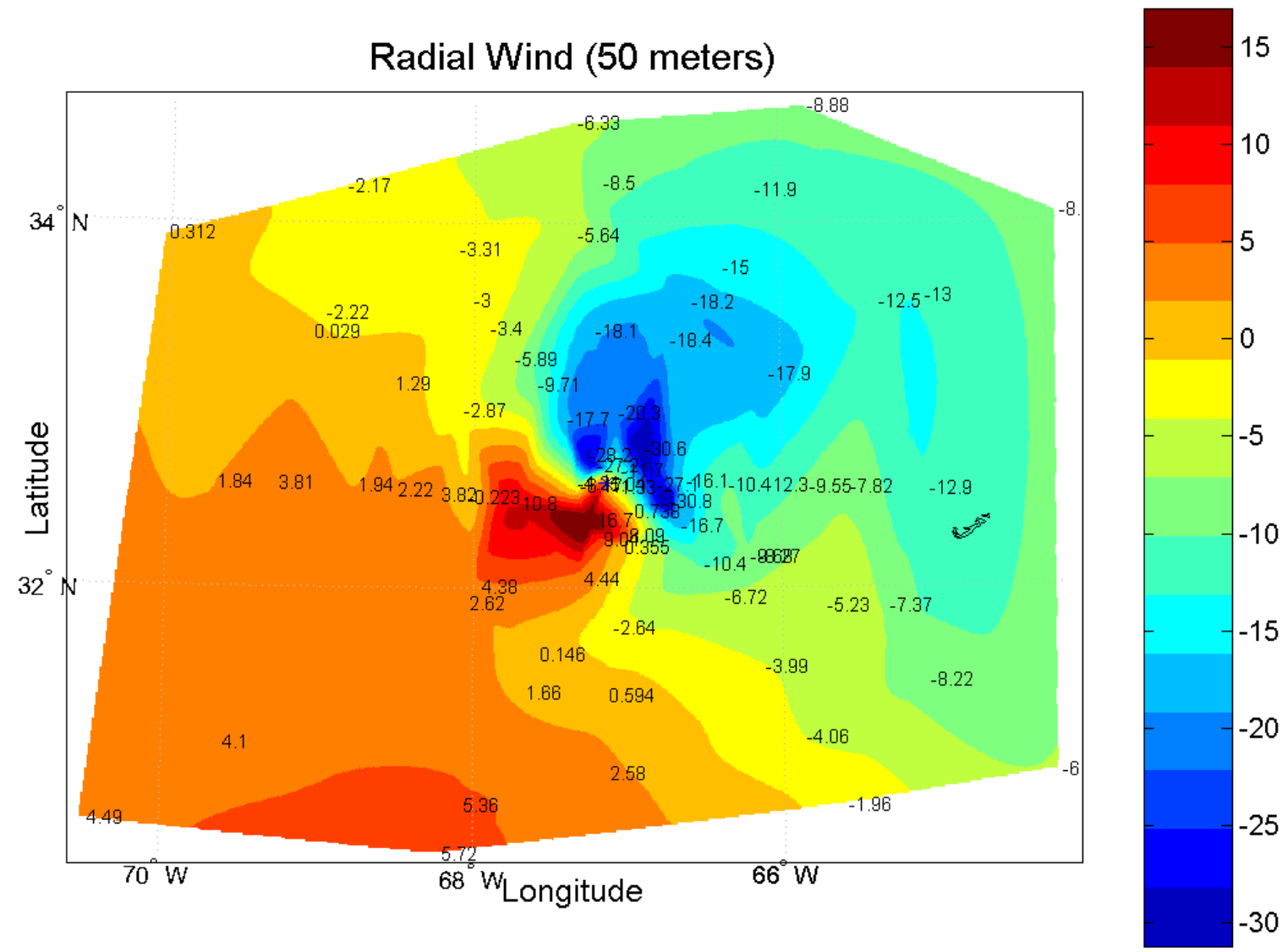

Figure 20. Plan view of the radial winds $\left(\mathrm{m} \mathrm{s}^{-1}\right)$ on the $23^{\text {rd }}$ of September, 2001, using the piecewise cubic Hermite interpolation. Numbers represent the exact values that each GPS sonde read. Color bar to the right denotes values given by the piecewise cubic Hermite interpolation. 


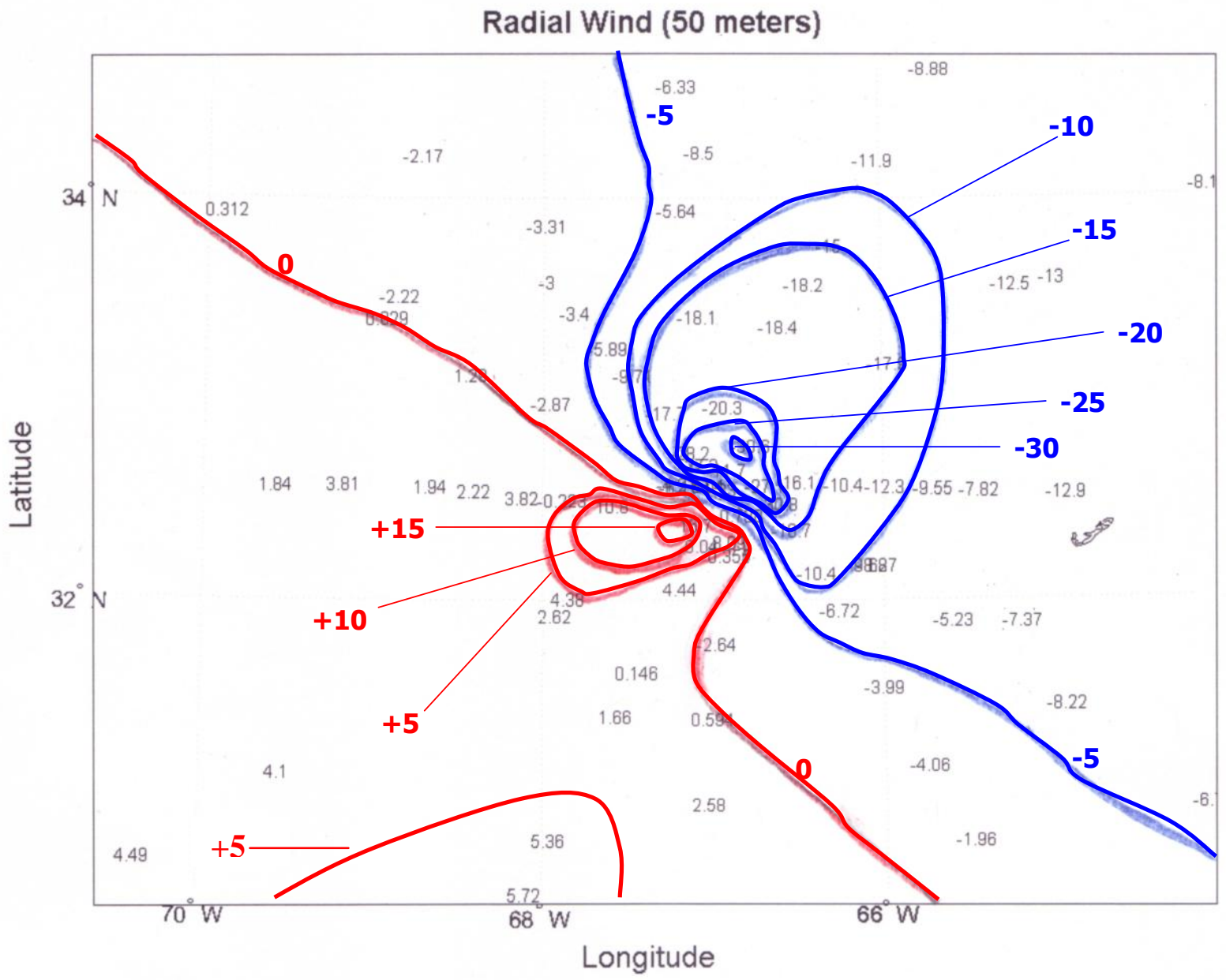

Figure 21. Plan view of the radial winds $\left(\mathrm{m} \mathrm{s}^{-1}\right)$ on the $23^{\text {rd }}$ of September, 2001. Analysis was done subjectively. Numbers represent the exact values that each GPS sonde read. Contours show values for subjective analysis. 


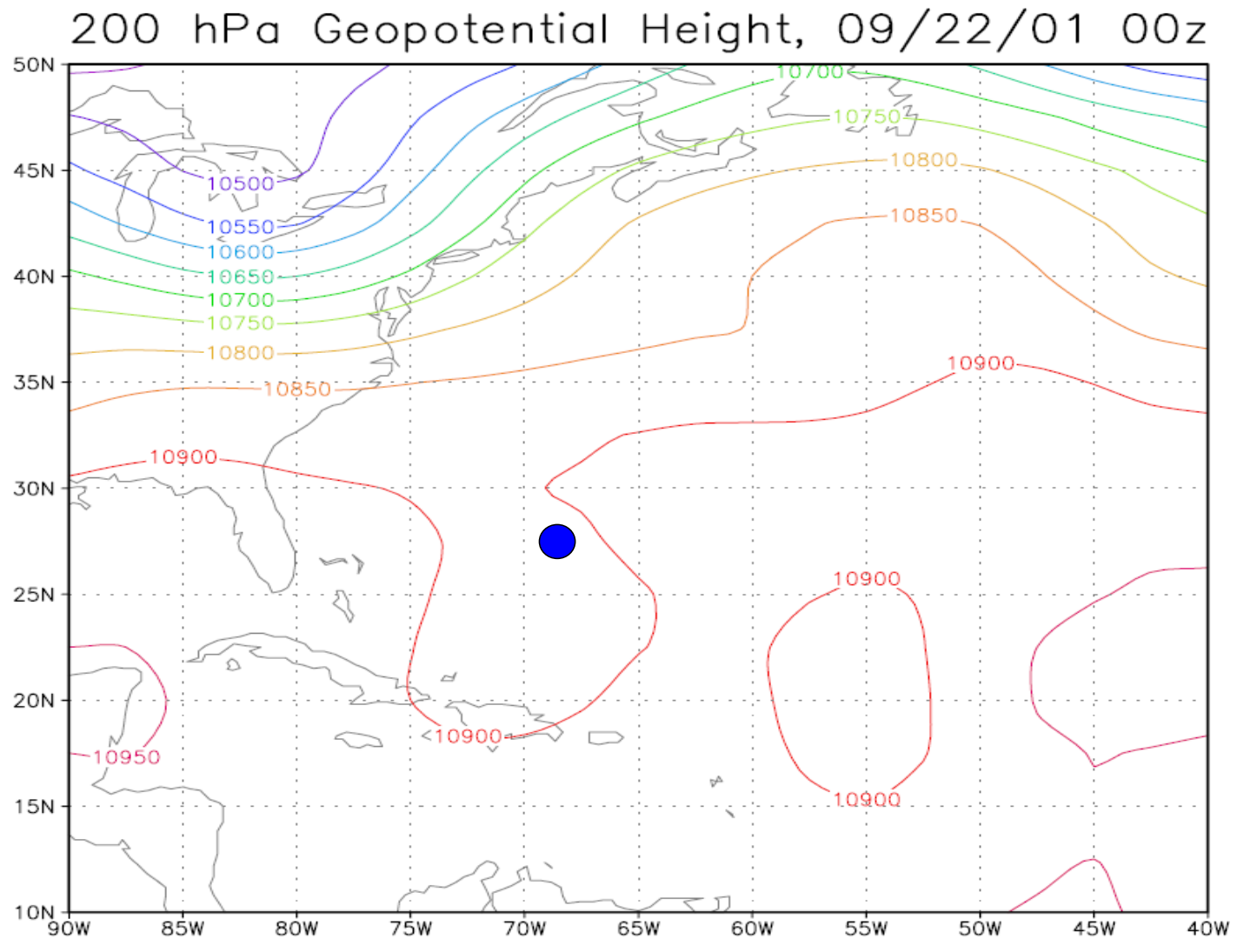

Figure 22. NCEP/NCAR Reanalysis $200 \mathrm{hPa}$ geopotential height field at $00 \mathrm{UTC}$ on September 22, 2001. Blue dot shows low-level circulation center. 


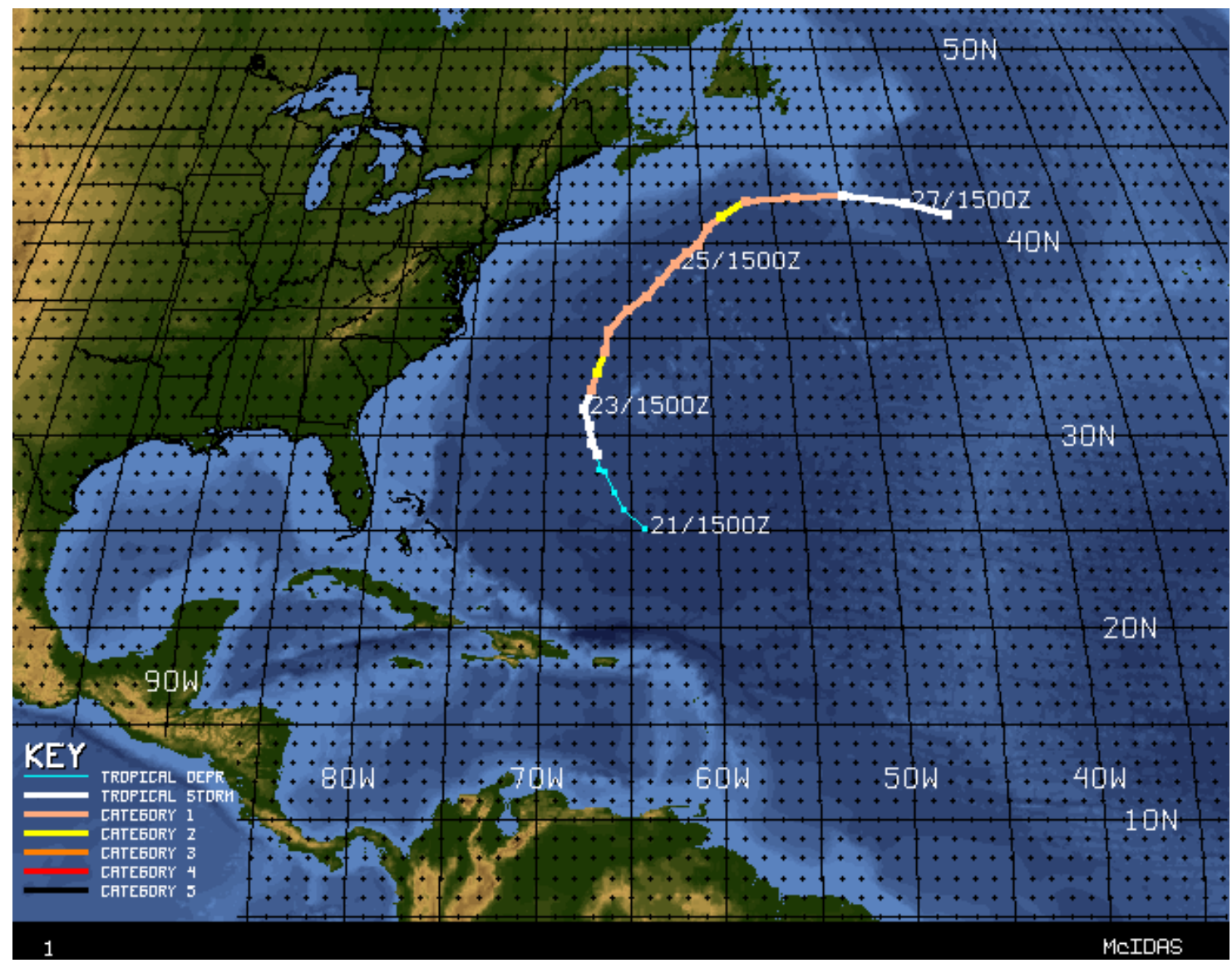

Figure 23. Track of Humberto from the time it became a tropical depression until it dissipated. Key shows Saffir-Simpson Scale. 


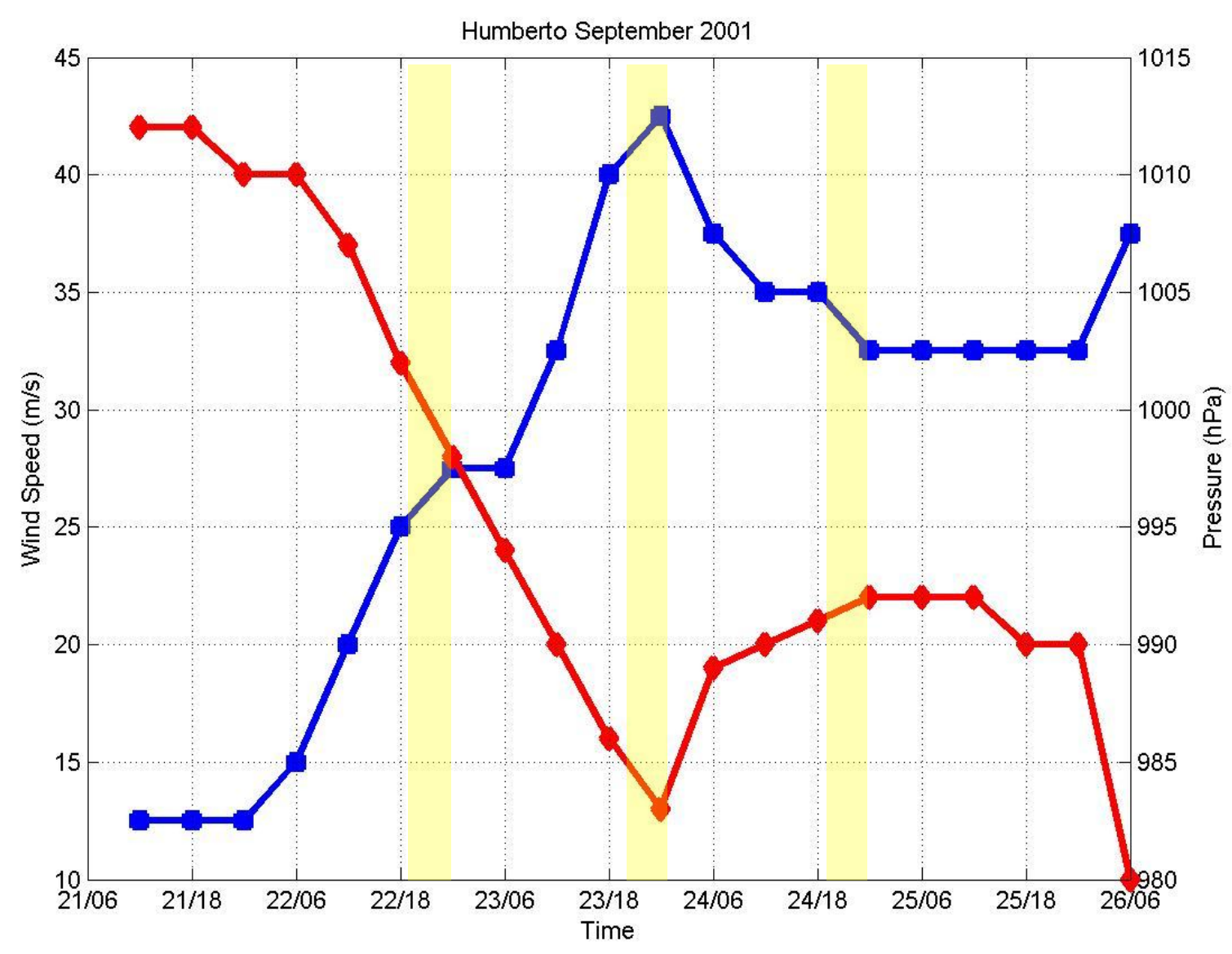

Figure 24. "Best Track" 1-minute sustained $10 \mathrm{~m}$ wind speed and surface pressure data for Humberto. Red line marks the pressure line with red diamonds showing the time of each observation. Blue line marks the $10 \mathrm{~m}$ wind speed with blue squares marking the time of each observation. Light yellow rectangles show times of observation for each of the three days. 
A

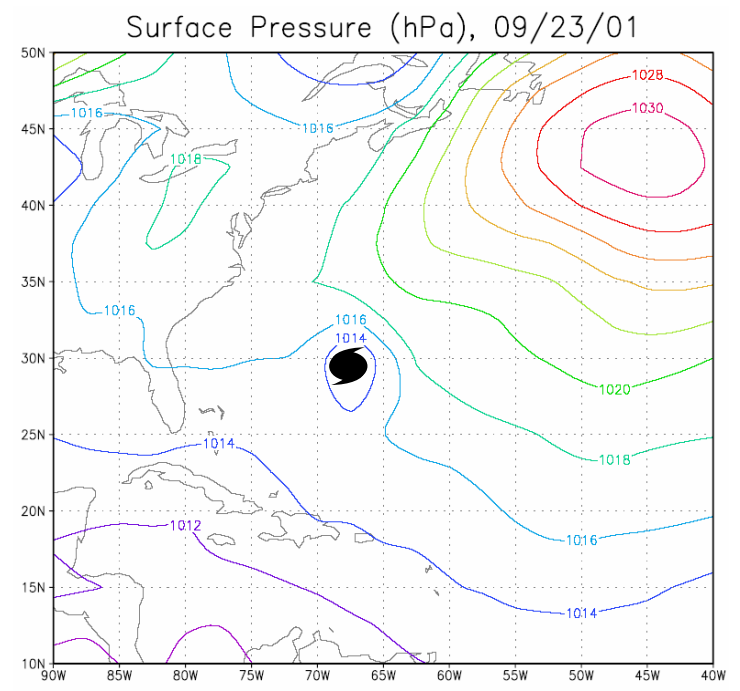

B

$500 \mathrm{hPa}$ Geopotential Height and Wind, 09/23/01

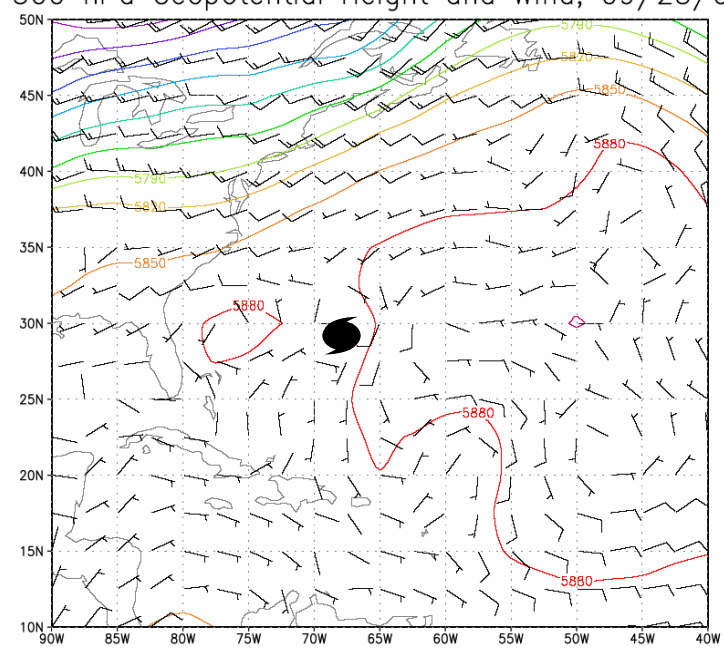

Figure 25. (A) NCEP/NCAR Reanalysis surface pressure chart (hPa) at 00 UTC on September $23^{\text {rd }}, 2001$. (B) NCEP/NCAR Reanalysis $500 \mathrm{hPa}$ geopotential height field with wind vectors. Time is 00 UTC on the $23^{\text {rd }}$ of September 2001. 


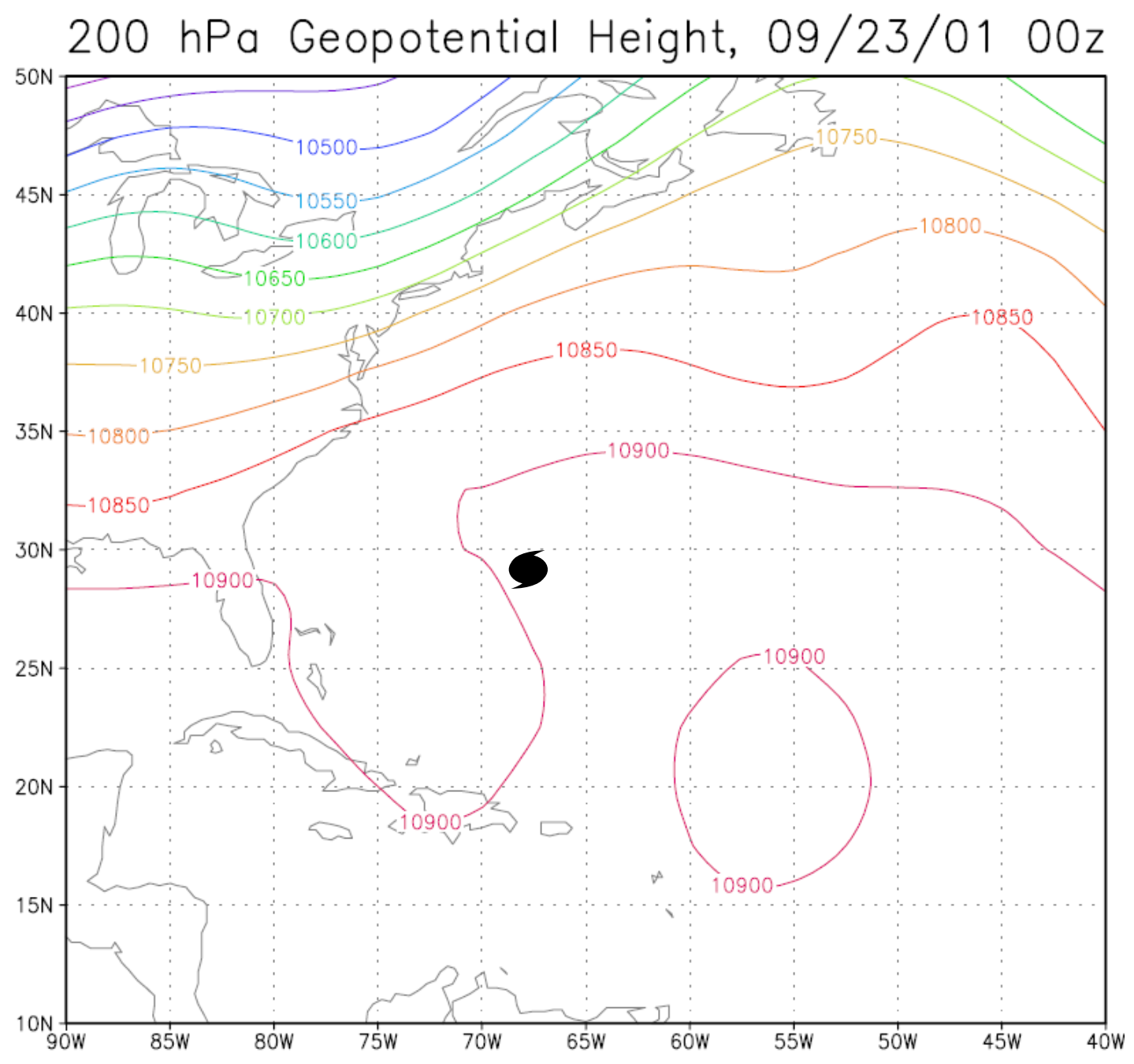

Figure 26. NCEP/NCAR Reanalysis $200 \mathrm{hPa}$ geopotential height field at $00 \mathrm{UTC}$ on September 23, 2001. 

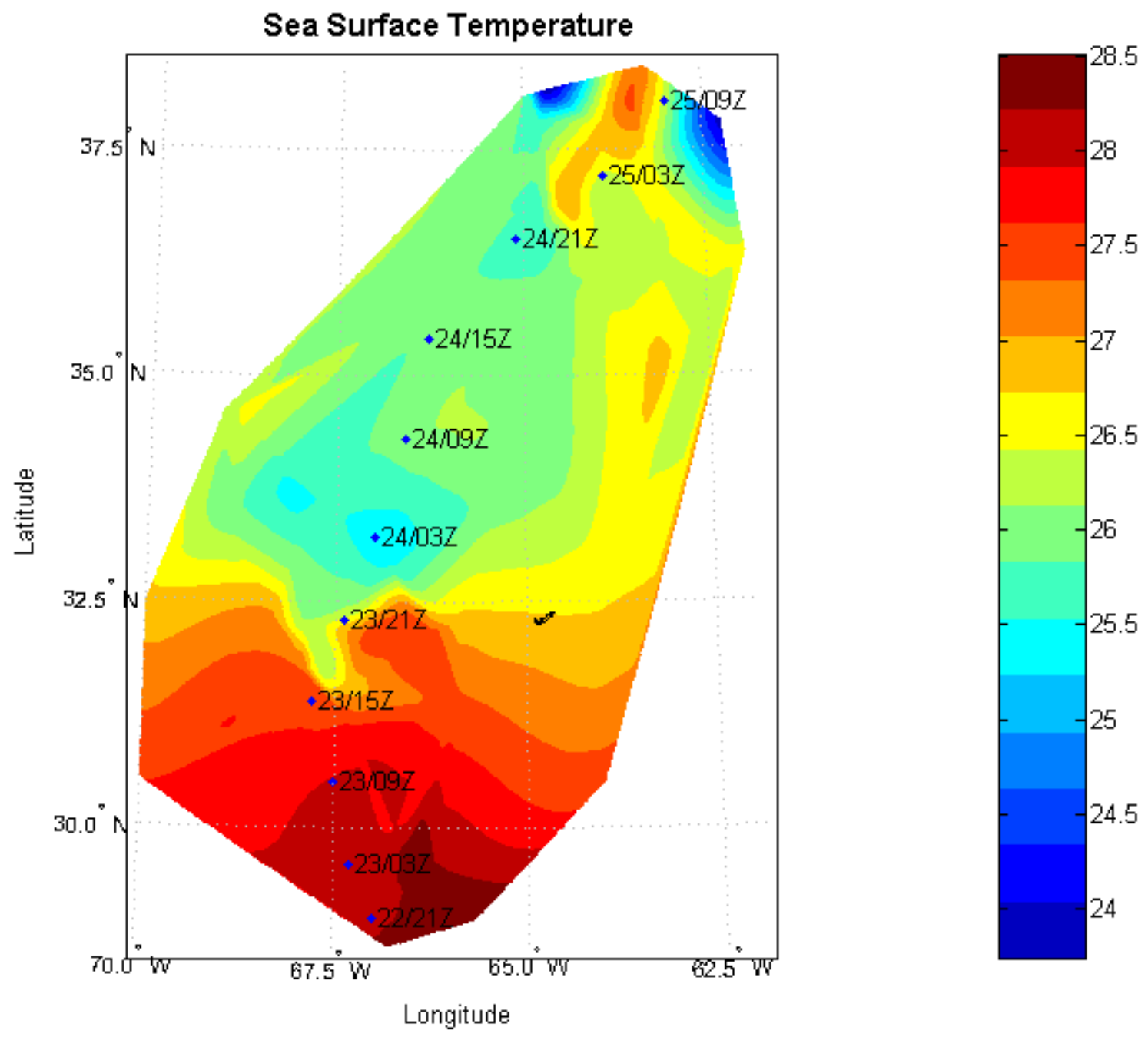

Figure 27. Map of sea surface temperatures $\left({ }^{\circ} \mathbf{C}\right)$ from the AXBT's. AXBT's were deployed from approximately 18:30 - 24:00 UTC on the $22^{\text {nd }}, 23^{\text {rd }}$, and $24^{\text {th }}$ of September. Blue dots represent the circulation center and the associated time. Color bar to the right denotes sea surface temperature. 


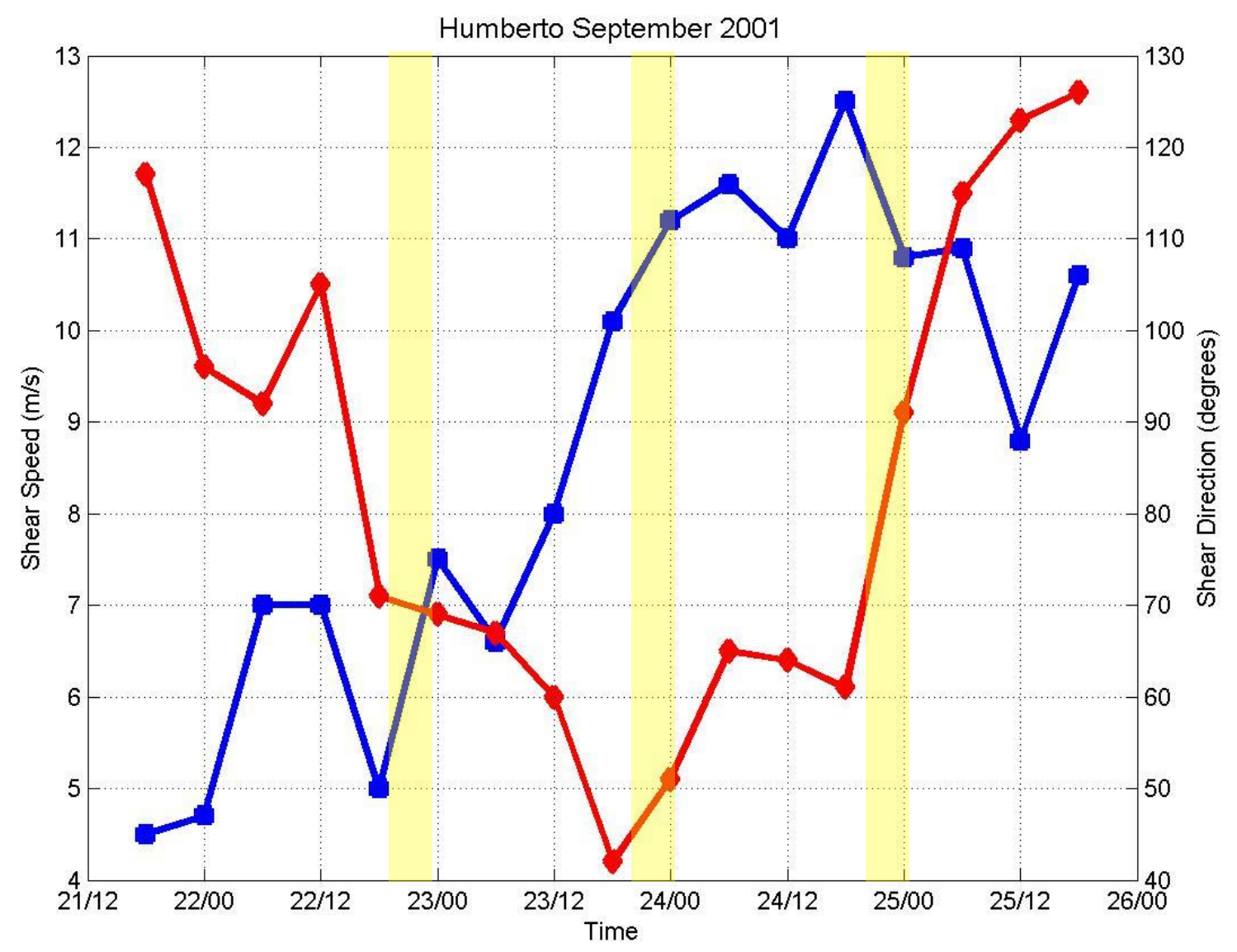

Figure 28. The magnitude and direction of the vertical shear of the horizontal wind from the SHIPs model. Blue line shows the vertical wind shear magnitude $\left(\mathrm{m} \mathrm{s}^{-1}\right)$. Red line displays the shear direction. Light yellow rectangles show times of observation for each of the three days. 


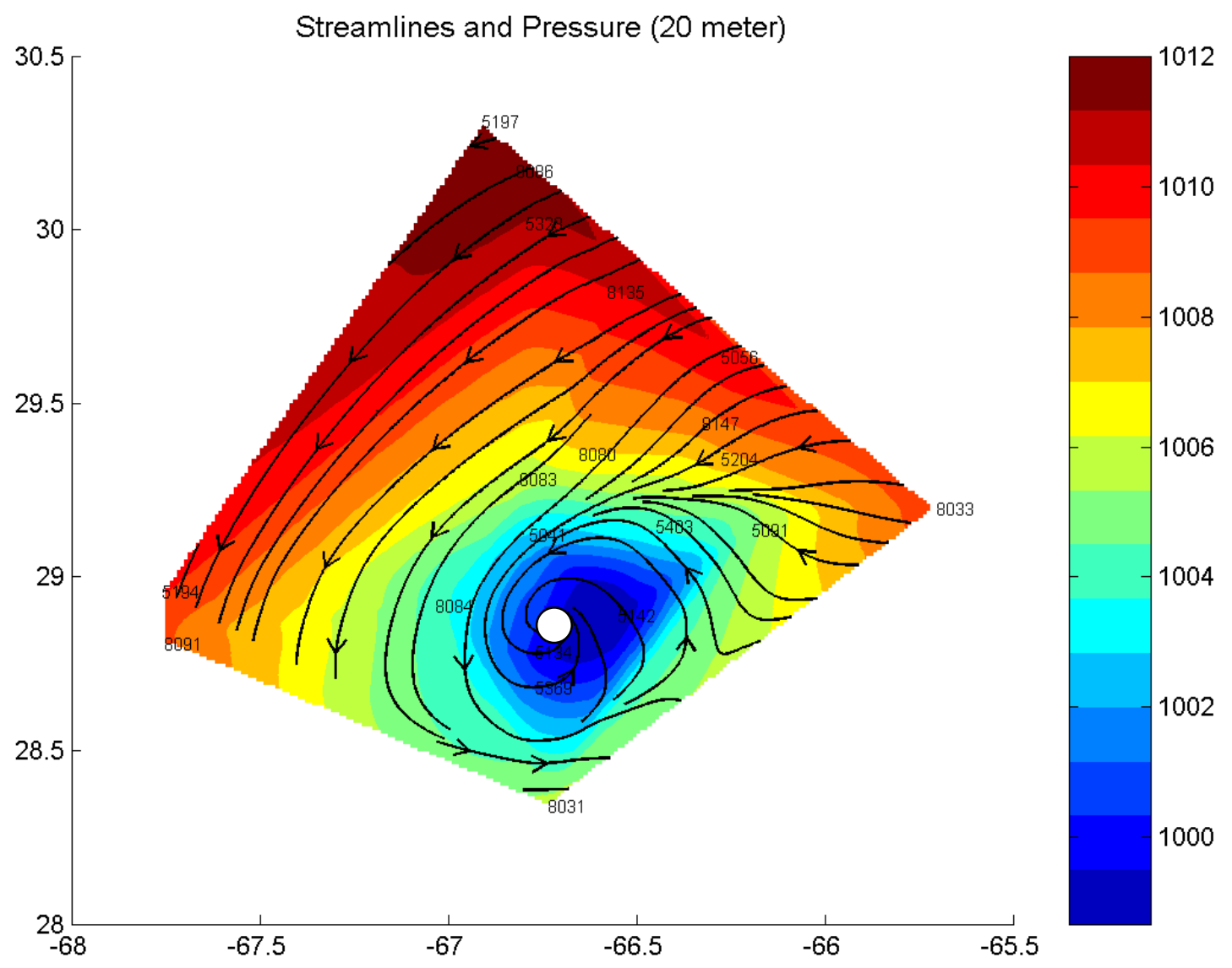

Figure 29. Time-space composite of GPS sondes. Latitude and longitude delineated every 0.5 degrees. Pressure $(\mathrm{hPa})$ is in color and streamlines are shown. Color bar to the right denotes pressure. White dot marks circulation center. Four digit numbers correspond to GPS sonde locations. 


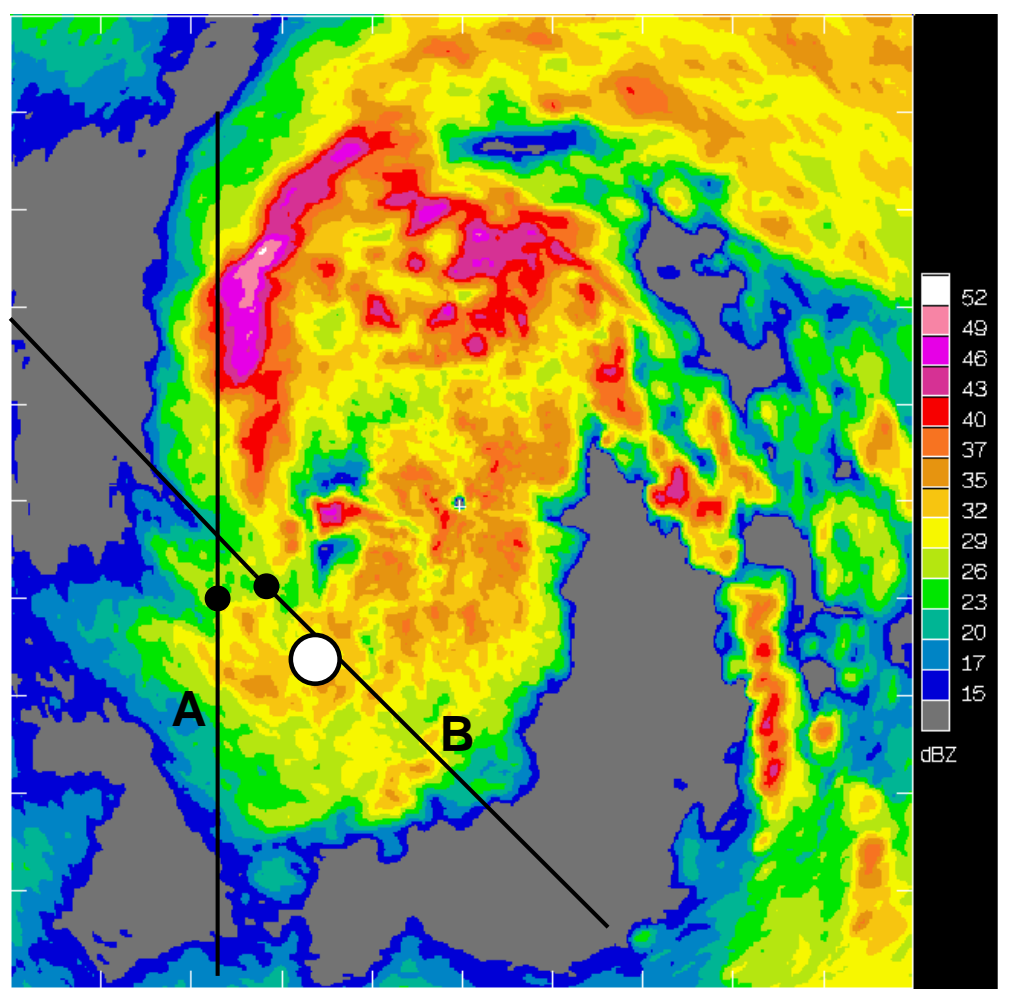

\section{9:00 Z}

$120 \times 120 \mathrm{~km}$

Figure 30. Plan view reflectivity image at 19:00 UTC on September $22^{\text {nd }}$. Image is $120 \mathrm{x}$ $120 \mathrm{~km}$. White dot shows the center of low pressure. Lines A and B are RHI reflectivity images shown in the next two figures. Two black dots show the position of the WP-3D at the time of the RHI scans. The WP-3D is moving east northeast, just north of the lowlevel circulation center. 

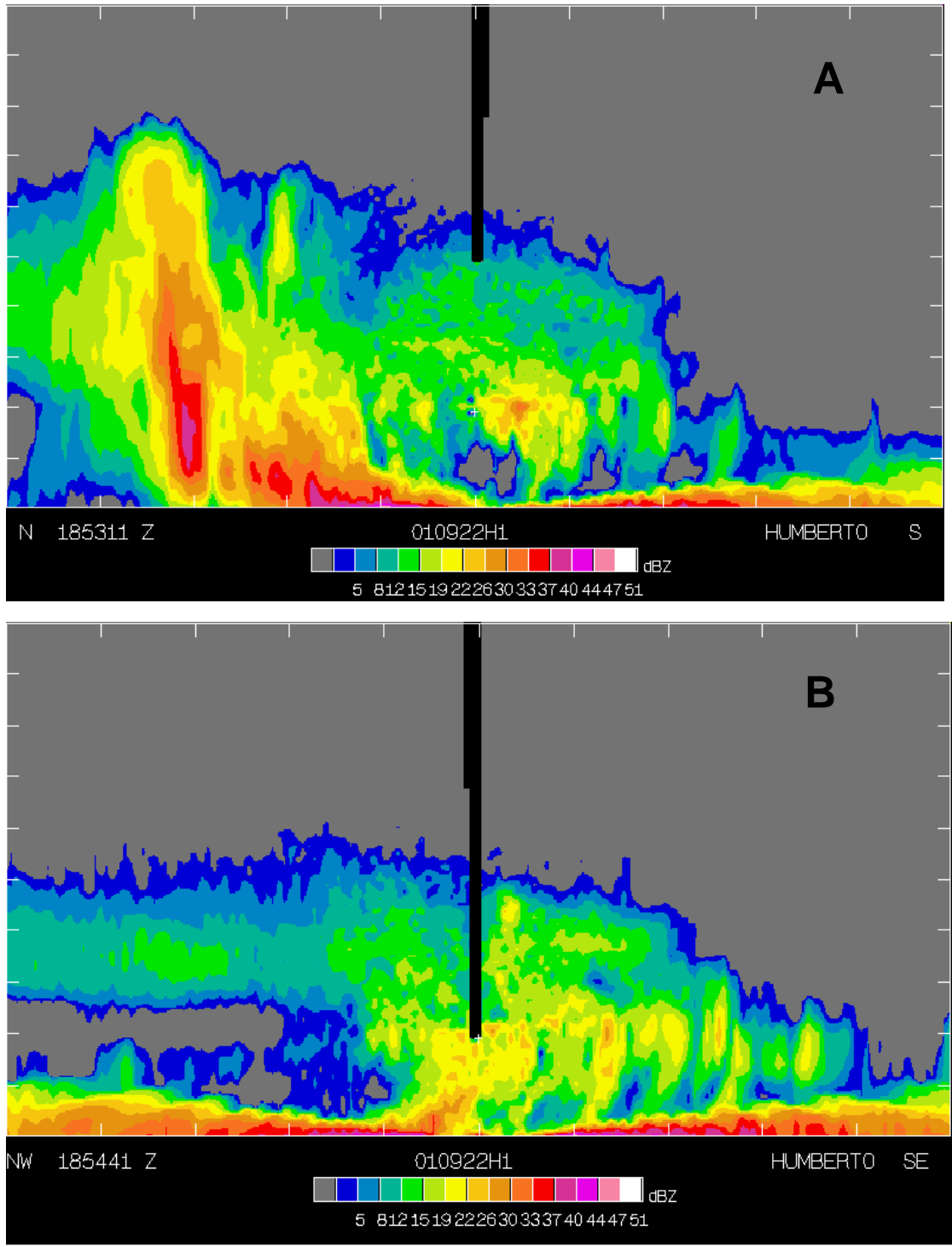

$120 \times 20$ km

Figure 31. (A) RHI reflectivity image at 18:53 UTC on September $22^{\text {nd }}, 2001$. Image is $120 \mathrm{~km}$ wide and $20 \mathrm{~km}$ high. Letter "A" corresponds to the line in figure 27 . (B) Same as "A" except time is 18:54 UTC. "B" corresponds to the line in Figure 27. 


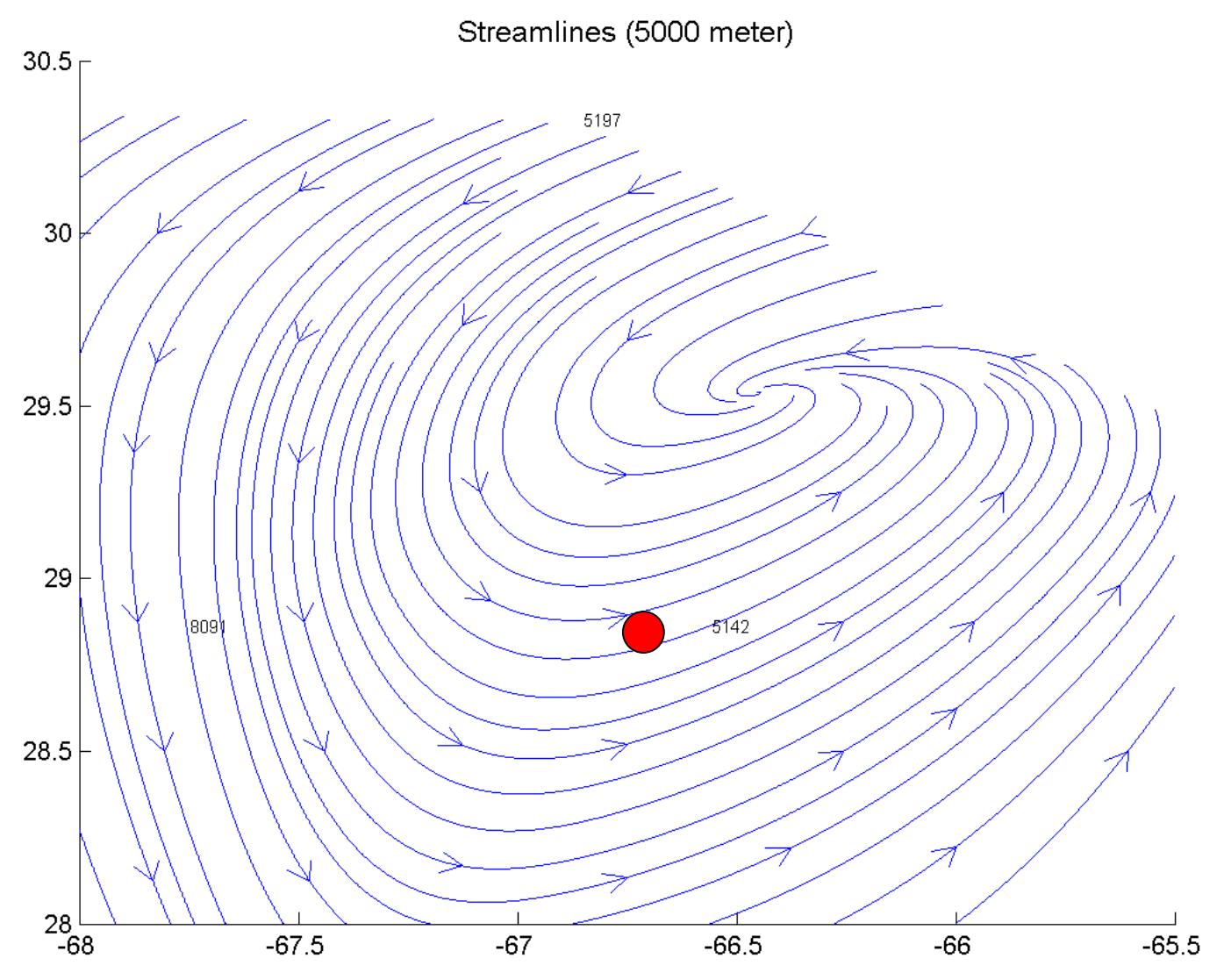

Figure 32. Streamlines at $5 \mathrm{~km}$ height as a function of latitude and longitude (degrees). Red dot corresponds to low-level pressure center. 


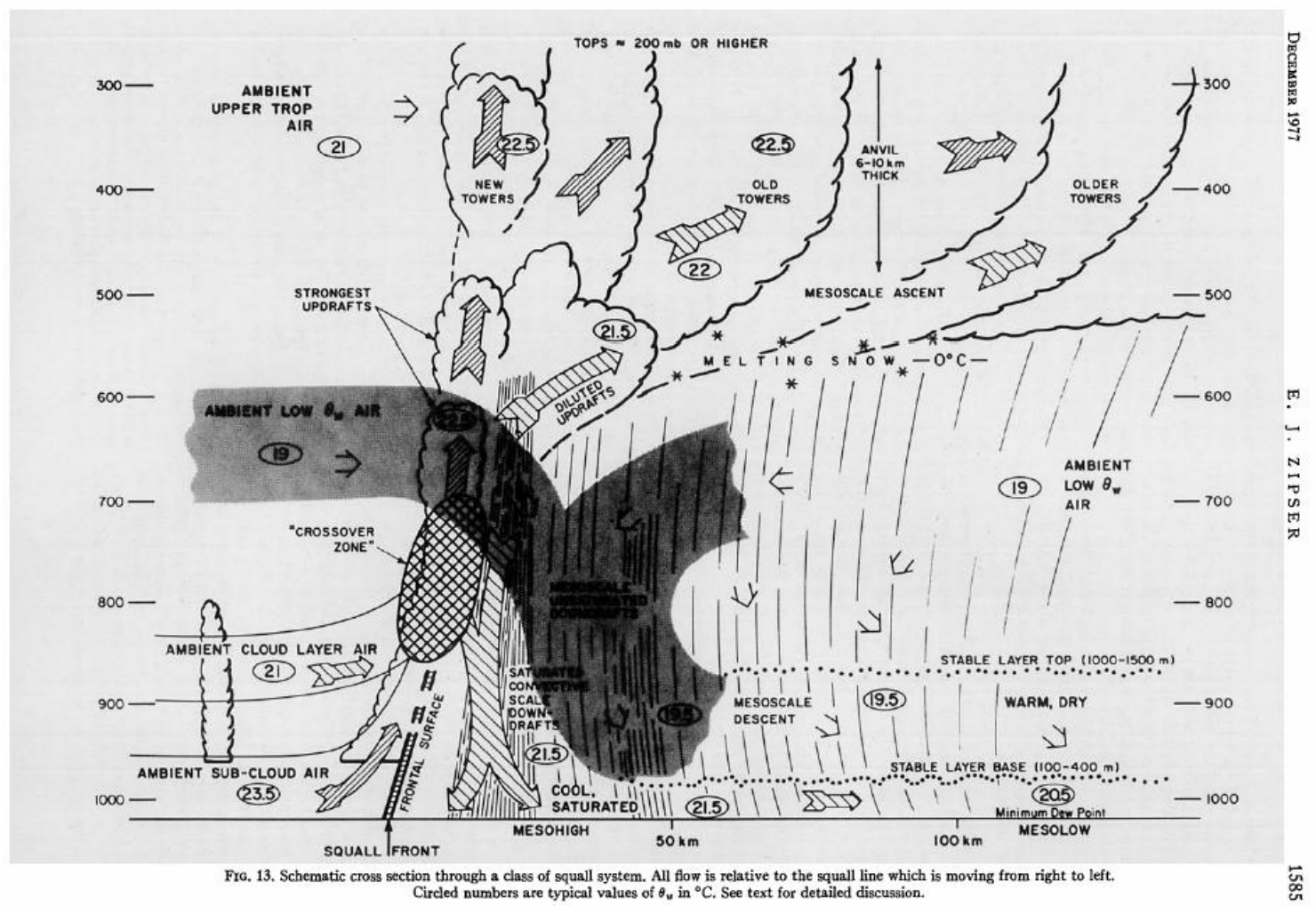

Figure 33. Schematic cross section through a class of squall system. All flow is relative to the squall line which is moving from right to left. Circled numbers are typical values of $\theta_{\mathrm{w}}$ in ${ }^{\circ} \mathrm{C}$ (Zipser 1977). 


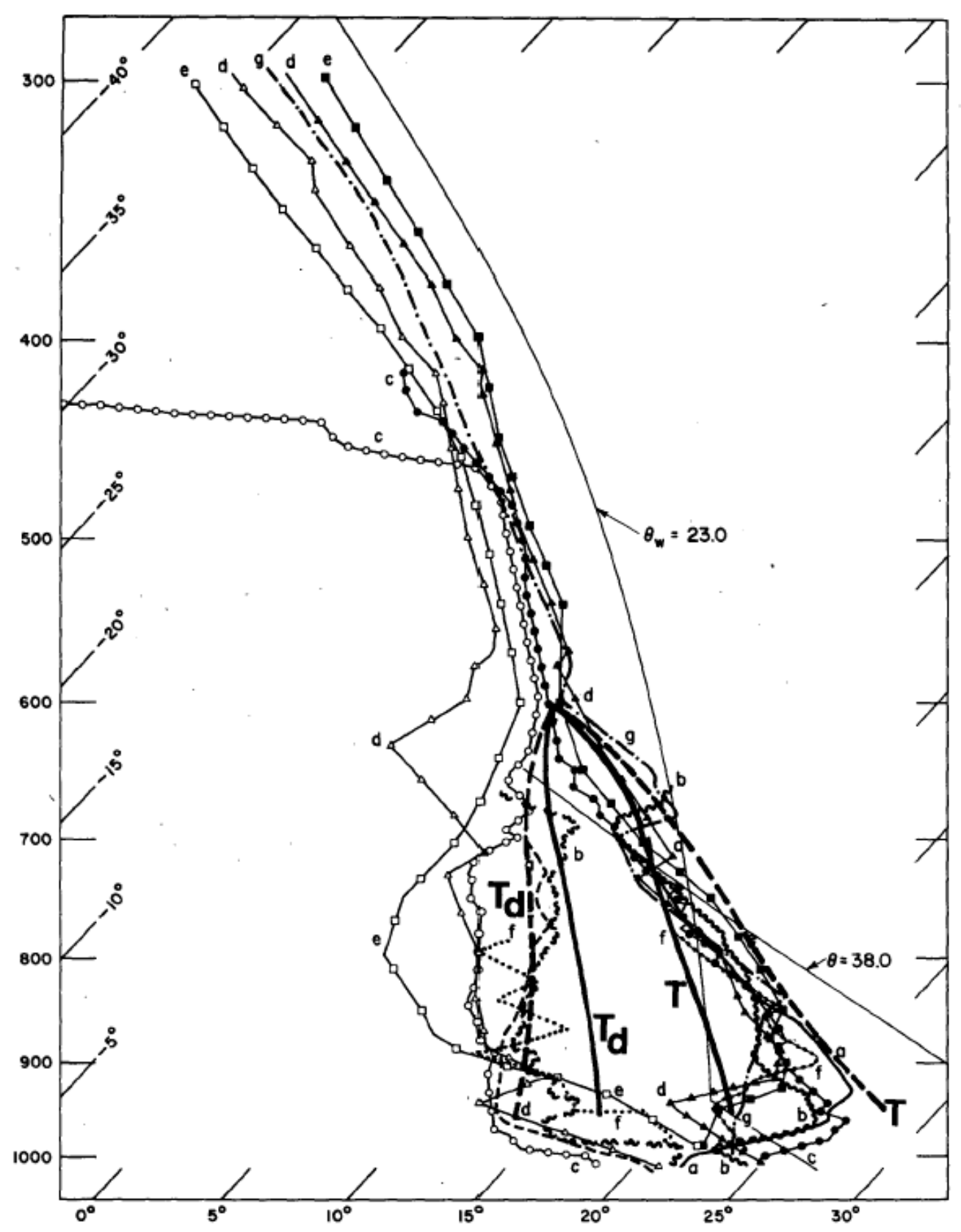

Figure 34. Skew-T $\log \mathrm{P}$ diagram of calculated profiles of temperature and dew point for mesoscale downdrafts originating at $600 \mathrm{mb}$ with a temperature of $0^{\circ} \mathrm{C}$, a relative humidity of $100 \%$, and vertical velocities of $0.2 \mathrm{~m} \mathrm{~s}^{-1}$ (heavy solid lines) and $0.5 \mathrm{~m} \mathrm{~s}^{-1}$ (heavy dashed lines) superposed on Zipser's (1977, Fig. 8) collection of soundings beneath anvil clouds. Vertical coordinate is pressure and all temperatures are in ${ }^{\circ} \mathrm{C}$. (Leary 1980). 


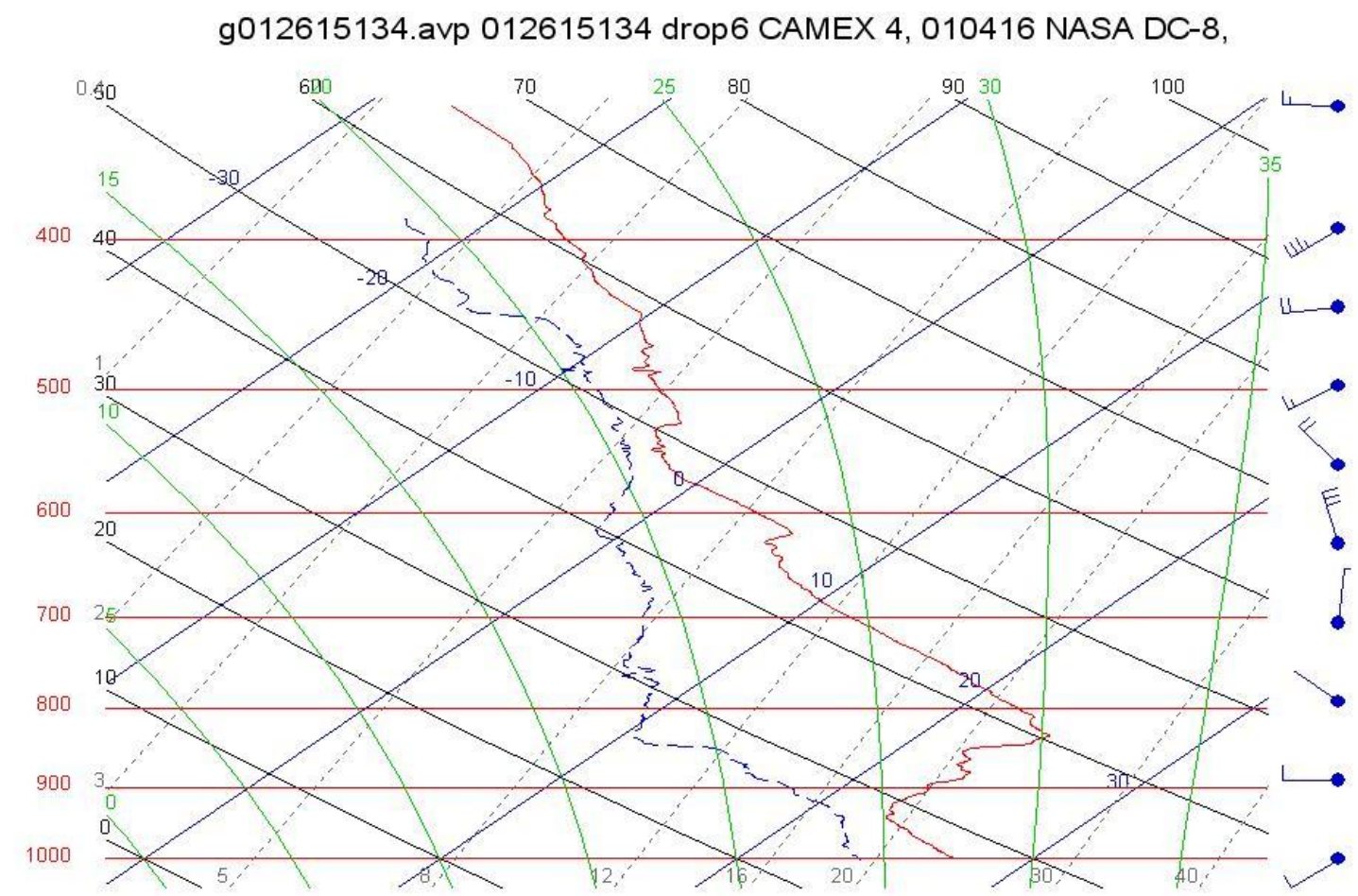

Aspen V2.2.1, 28 Mar 2005 20:50 UTC

Figure 35. Same as Fig. 17. GPS sonde jettisoned at 18:54 UTC on September $22^{\text {nd }}$, from the DC-8, near the surface circulation center. 
g011118104.avp 011118104 none CAMEX 4, CAMEX 4 - TD10 22Sep2001 Lockheed ER-2, NASA 809

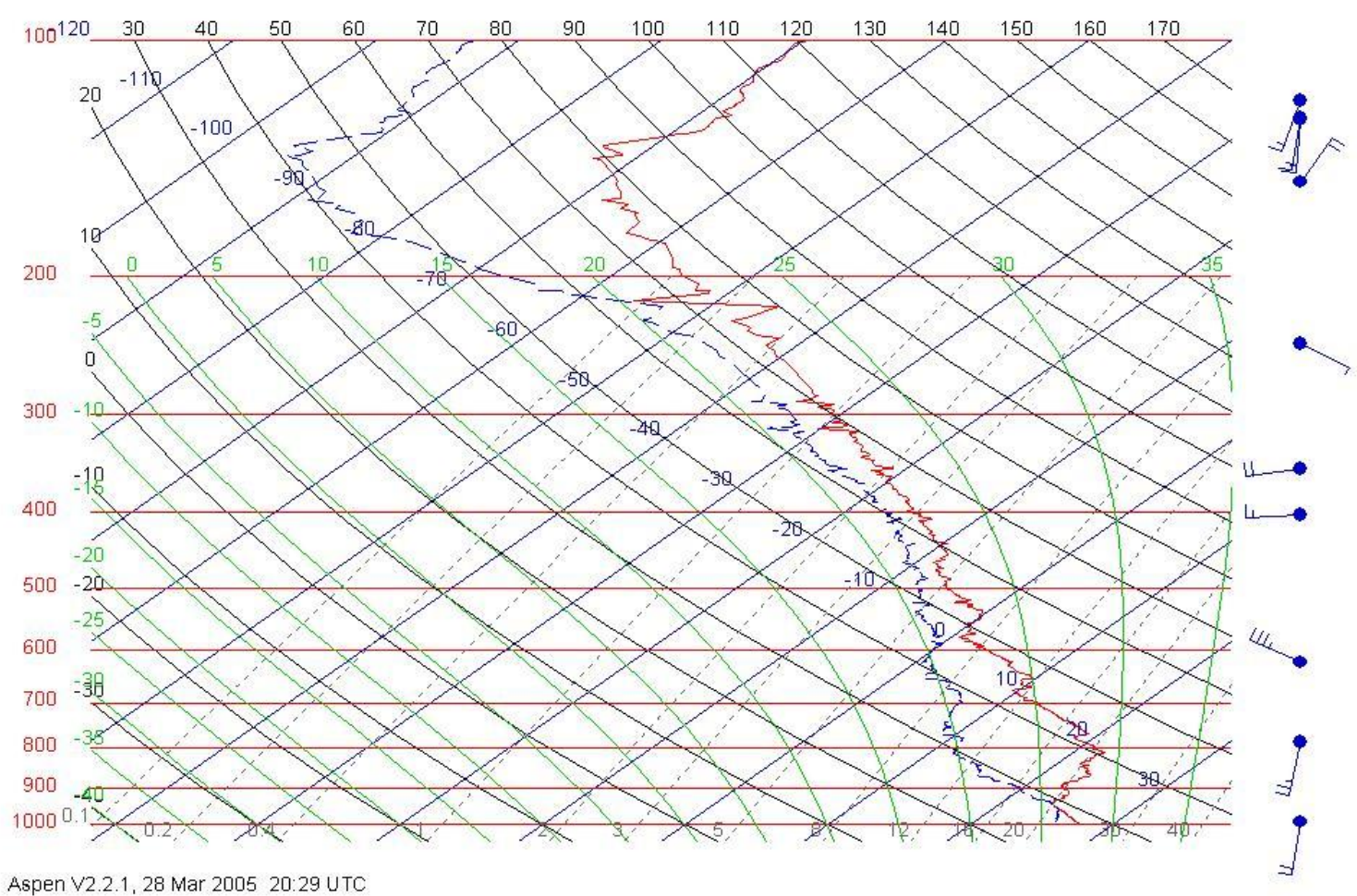

Figure 36. Same as Fig. 17. GPS sonde jettisoned at 21:19 UTC on September $22^{\text {nd }}$, from the ER-2, near the surface circulation center. 


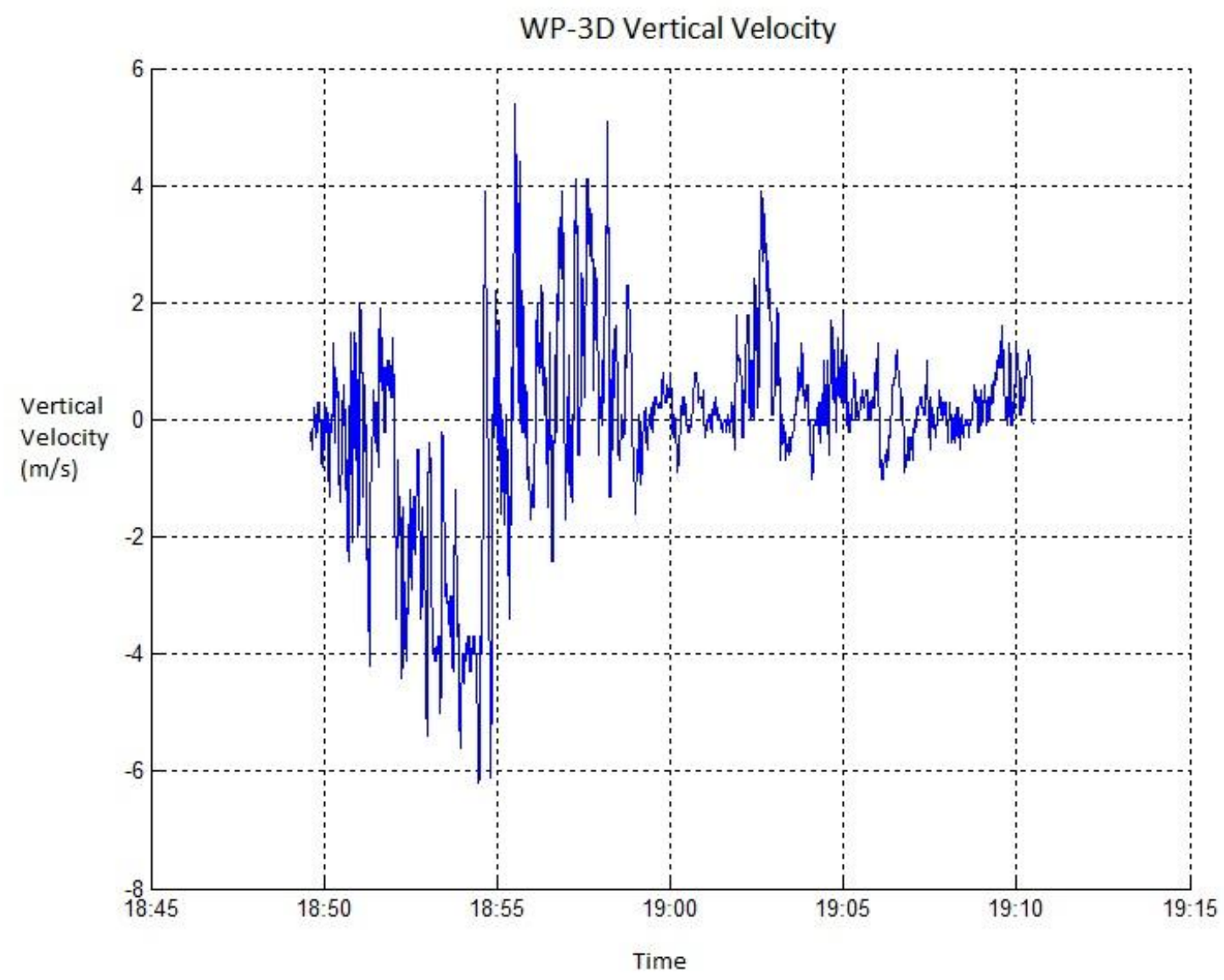

Figure 37. Vertical velocity $\left(\mathrm{m} \mathrm{s}^{-1}\right)$ as a function of time (UTC) from the WP-3D flying at $5 \mathrm{~km}$. 


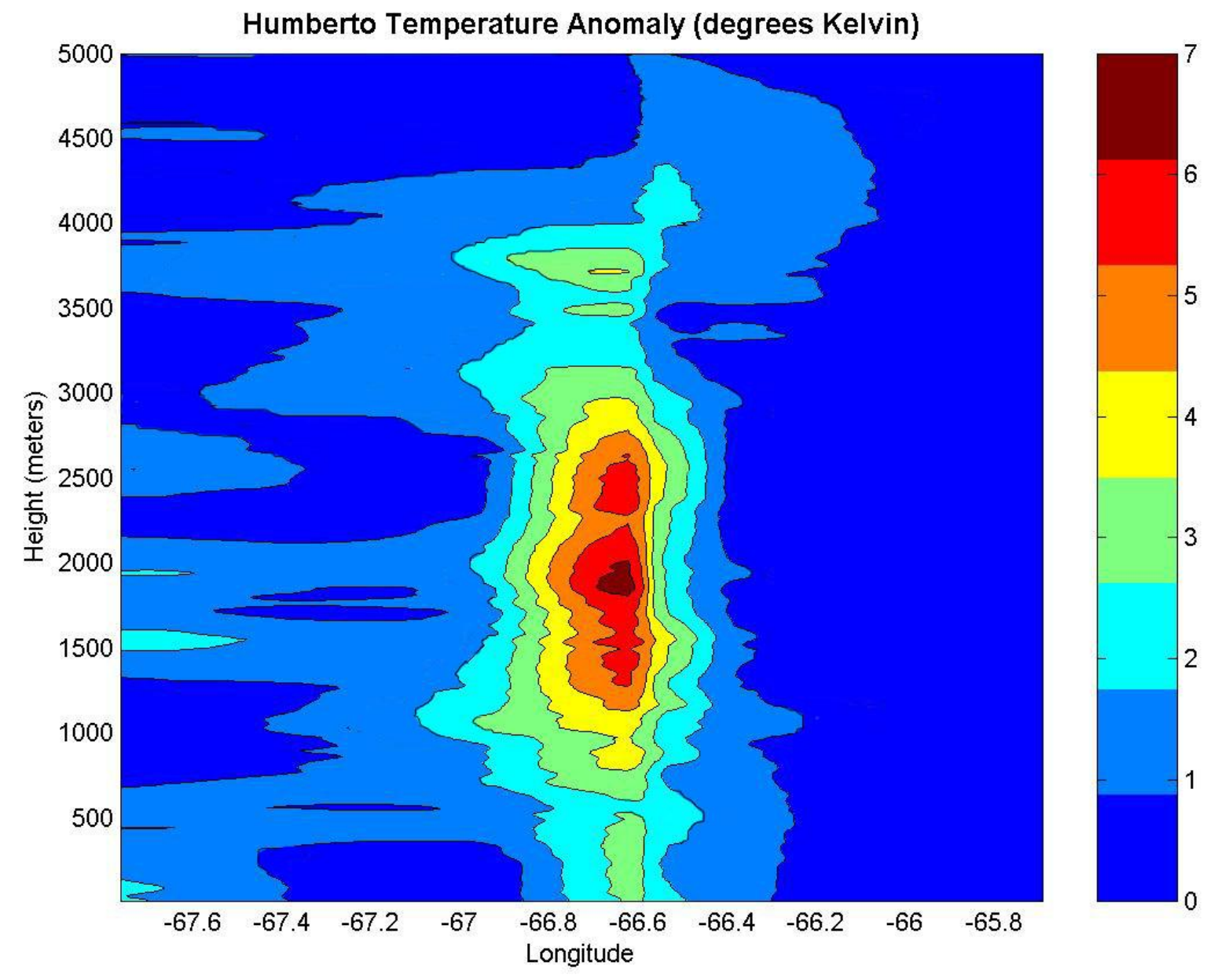

Figure 38. East-west vertical cross-section of the temperature anomaly, on an axis through the surface circulation center. Color bar indicates the temperature anomaly in degrees Kelvin. Storm center is located at $66.7^{\circ} \mathrm{W}$. 
Temperature at $400 \mathrm{hPa}$ (Celsius)

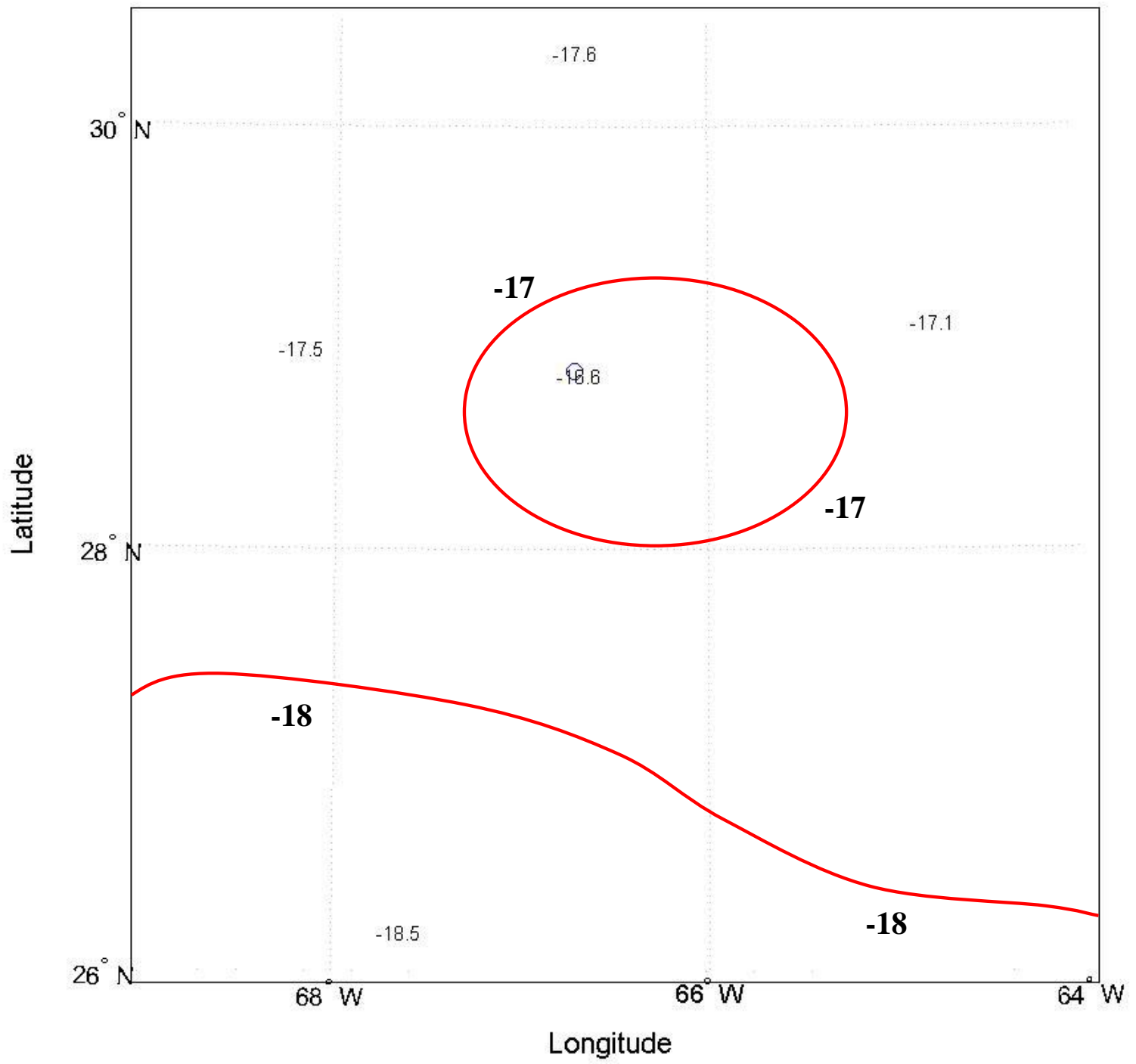

Figure 39. Plan view of temperature at $400 \mathrm{hPa}$ in degrees Celsius from the GPS sondes. Temperature contoured (solid red) every degree. Black circle marks low-level circulation center. 


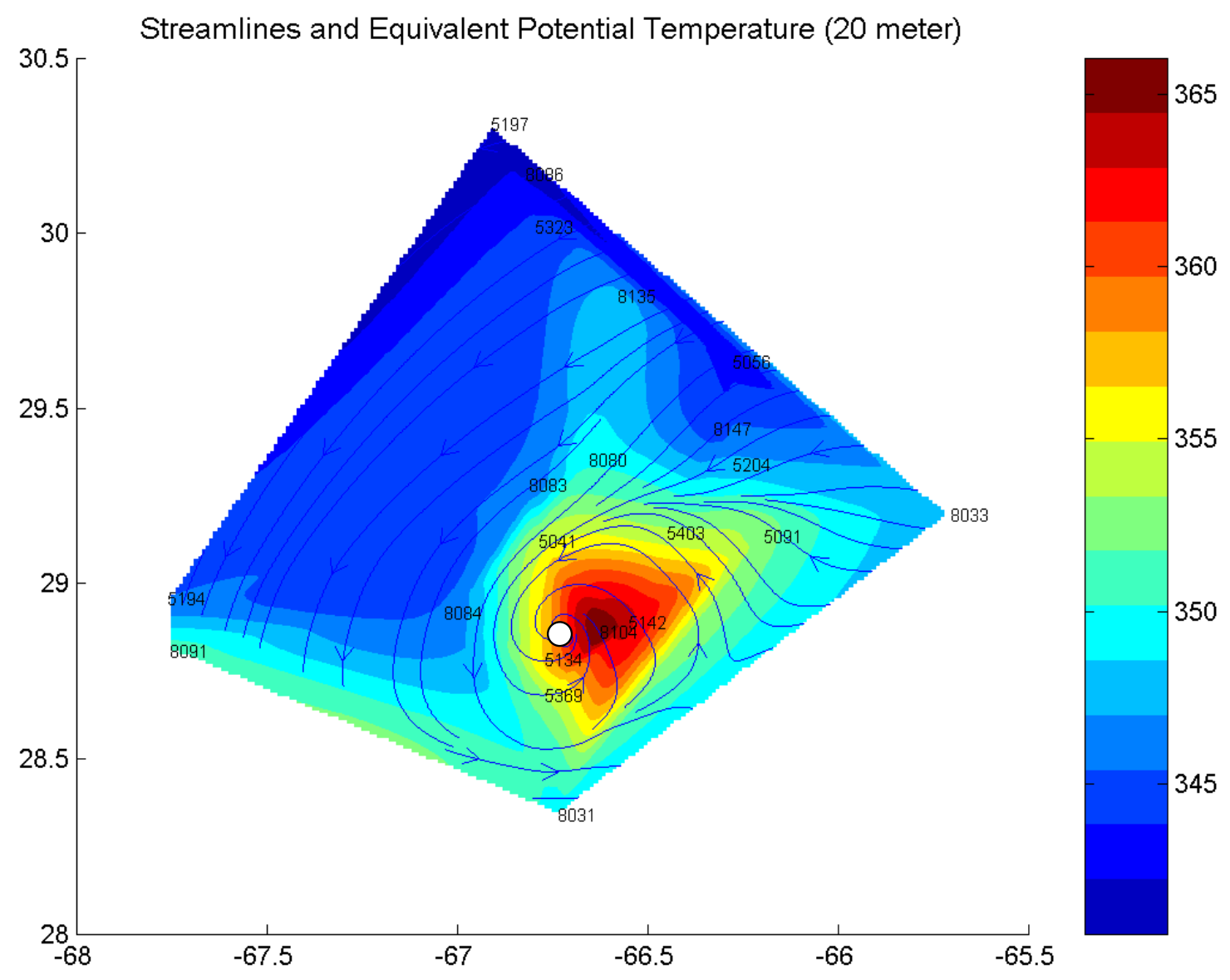

Figure 40. Plan view at 20 meter height of equivalent potential temperature. Streamlines superposed as blue lines with arrows. Latitude and longitude delineated every 0.5 degrees. Color bar at right corresponds to equivalent potential temperature (Kelvin). White dot indicates surface circulation center. 


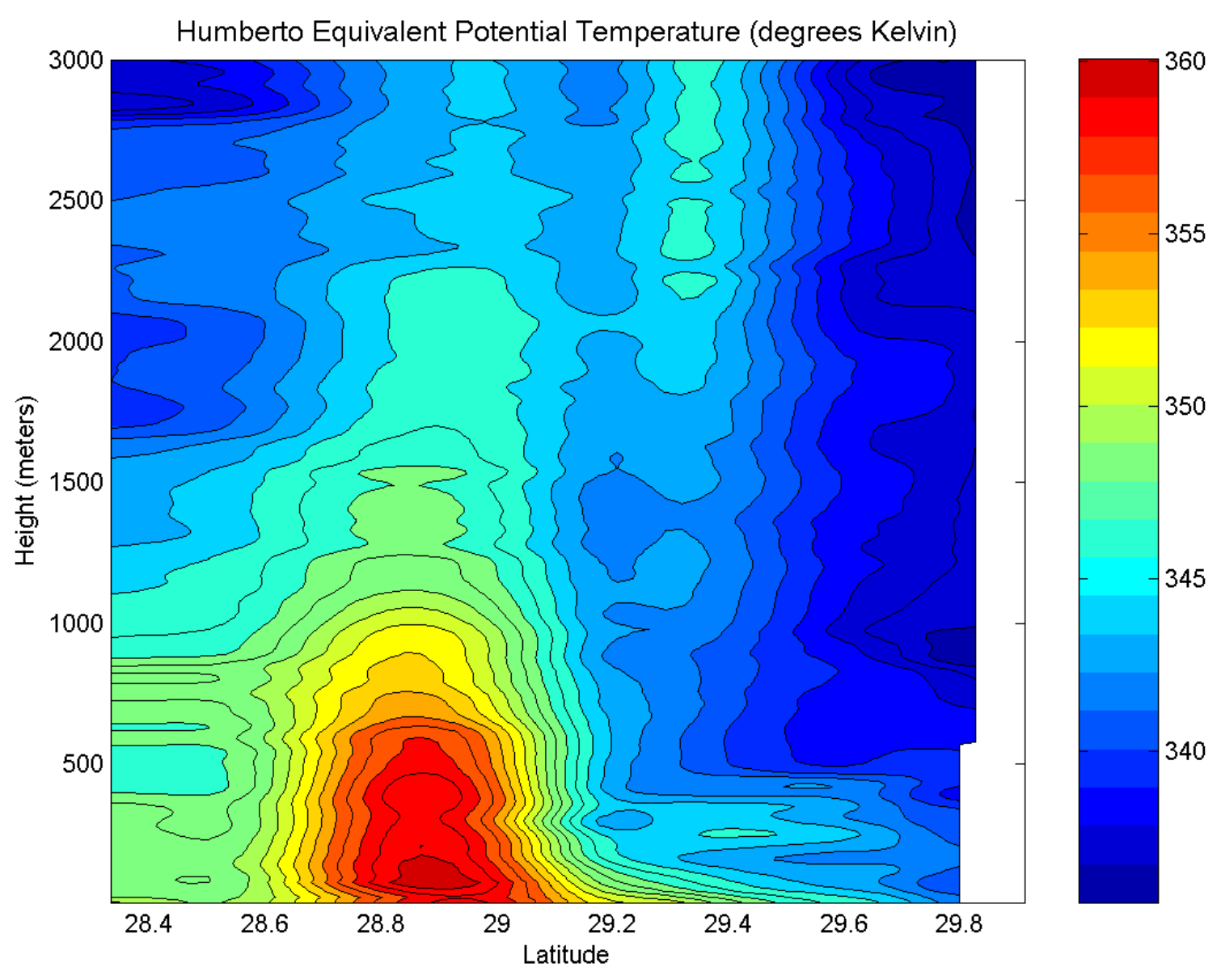

Figure 41. Vertical cross-section of equivalent potential temperature, taken $20^{\circ}$ clockwise of a north-south axis, through the surface circulation center. Color bar to right corresponds to equivalent potential temperature (Kelvin). Low-level circulation center is located at $28.9^{\circ} \mathrm{N}$. 

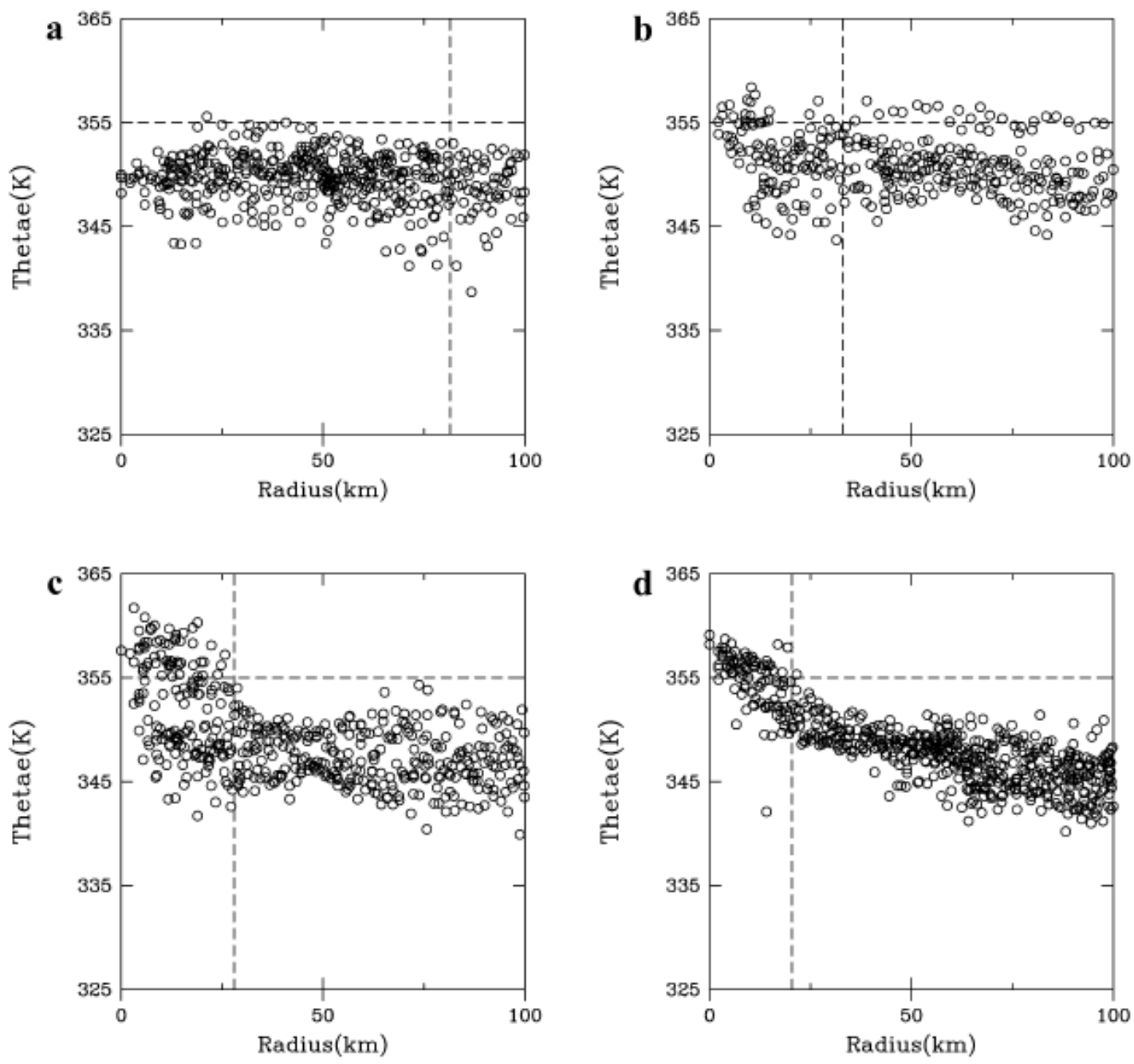

Figure 42. Radial scatterplots of equivalent potential temperature (K) calculated from the U.S. Air Force reconnaissance flight data with 30-s time resolution. All values are from elevations between 200 and 500 m: (a) 1600-2300 UTC 16 Jul, during depression stage; (b) 1400-2000 UTC $17 \mathrm{Jul}$, during early tropical storm stage when there were multiple vortex interactions; (c) 2200 UTC 17 Jul-0600 UTC 18 Jul, during late tropical storm stage; and (d) 1100-2100 UTC 18 Jul, the first full flight at hurricane intensity. The vertical dashed line indicates the radius of maximum wind taken from an average of the four-six estimates during each flight by reconnaissance aircraft. The value in (c) is averaged from the flights on either side. The horizontal line lies at $\theta_{\mathrm{e}}=355 \mathrm{~K}$. (Molinari et al. 2004). 


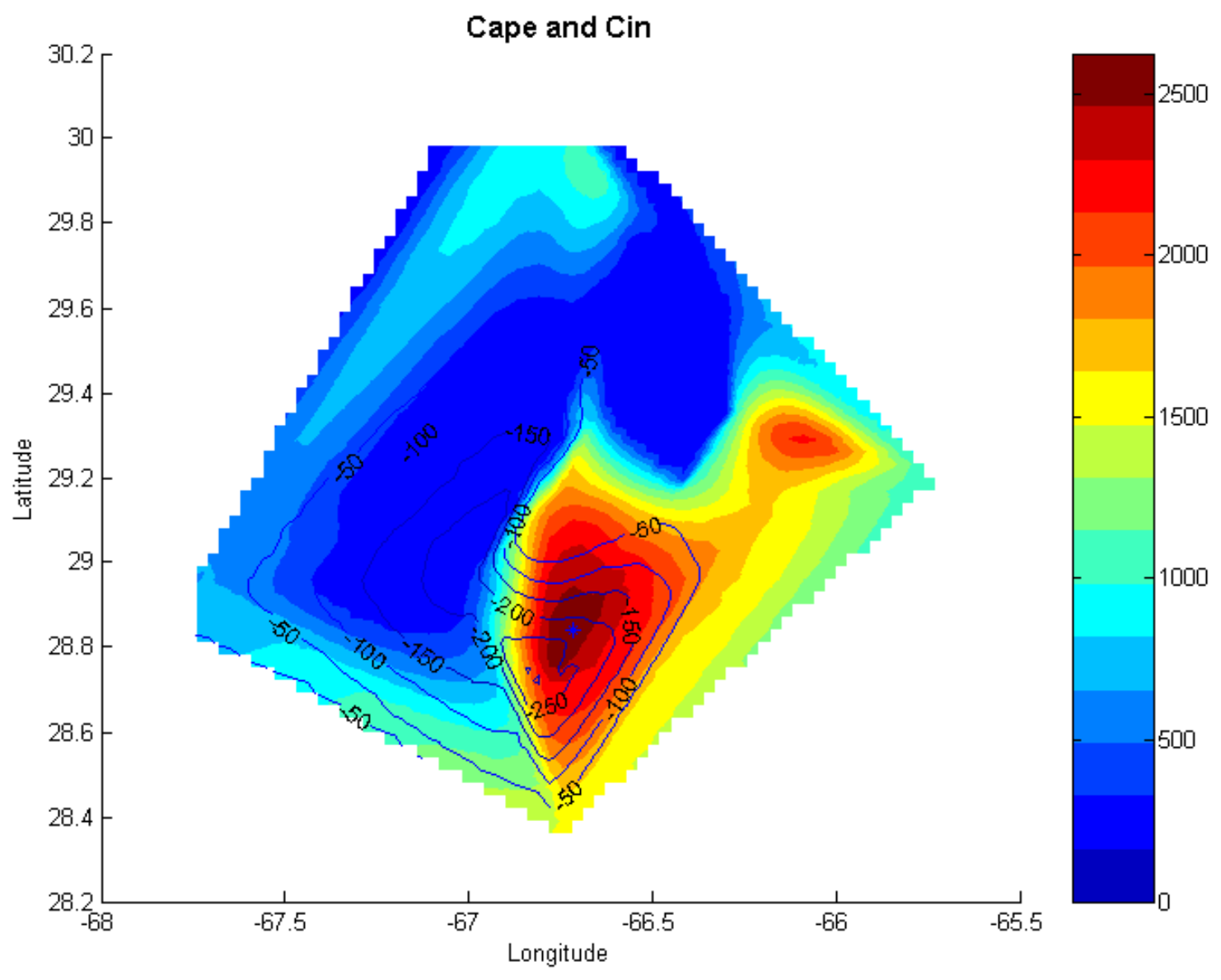

Figure 43. Plan view of CAPE $\left(\mathrm{J} \mathrm{kg}^{-1}\right)$ and $\mathrm{CIN}\left(\mathrm{J} \mathrm{kg}^{-1}\right)$. Latitude and longitude delineated every 0.5 degrees. CAPE is shown by the values on the color bar. CIN is represented by the contours. Circulation center is marked by a blue " $x$ ". 


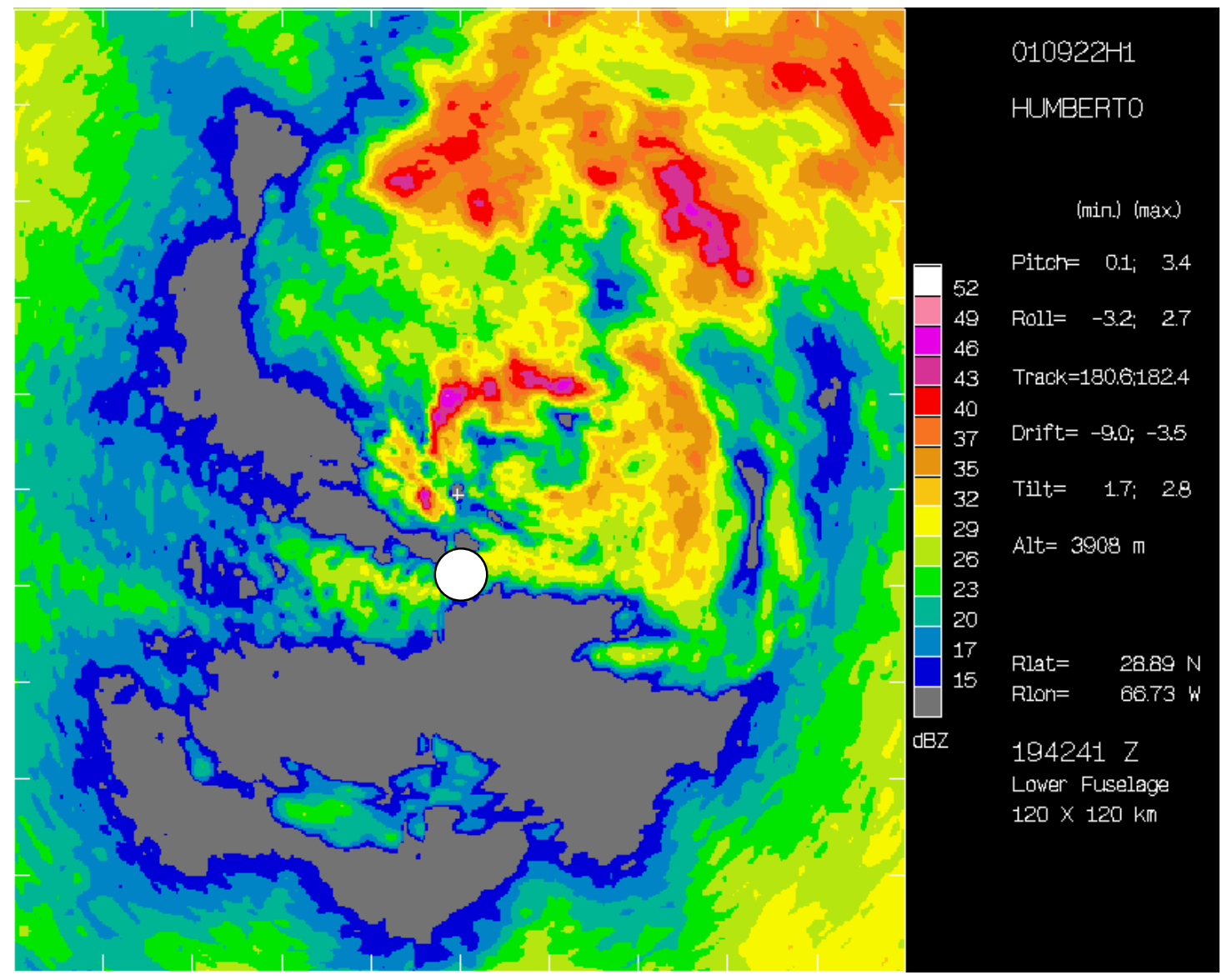

Figure 44. Plan view reflectivity image at 19:42 UTC on September $22^{\text {nd }}$. Image is 120 $\mathrm{x} 120 \mathrm{~km}$. White dot shows the low-level circulation center. Location of the WP-3D is marked by the white plus symbol in the center of the image. 


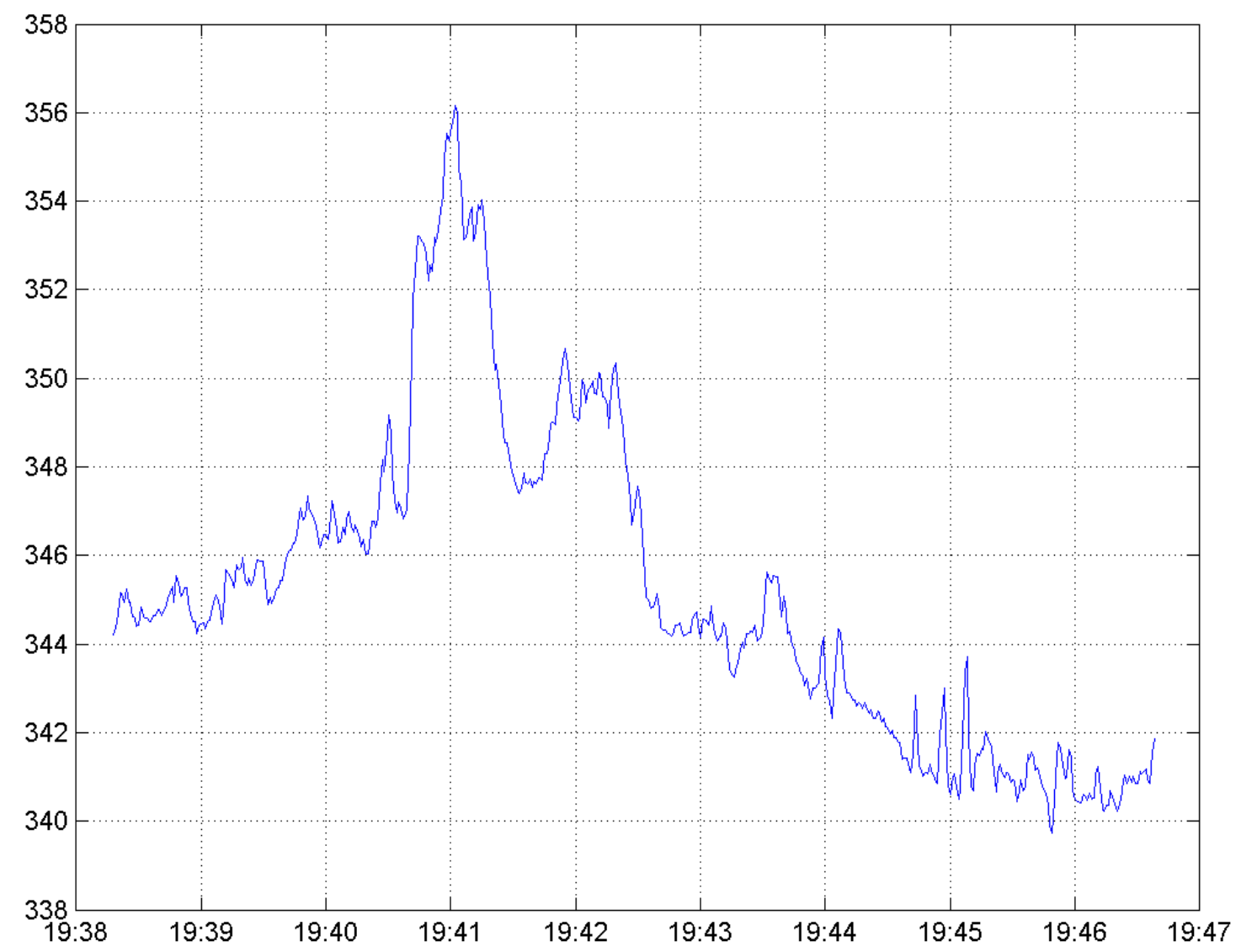

Figure 45. Equivalent potential temperature as measured from the NOAA WP-3D from 19:38 UTC through 19:47 UTC on $9 / 22 / 01$. Y axis is the equivalent potential temperature in degrees Kelvin and the $\mathrm{x}$ axis is time. 


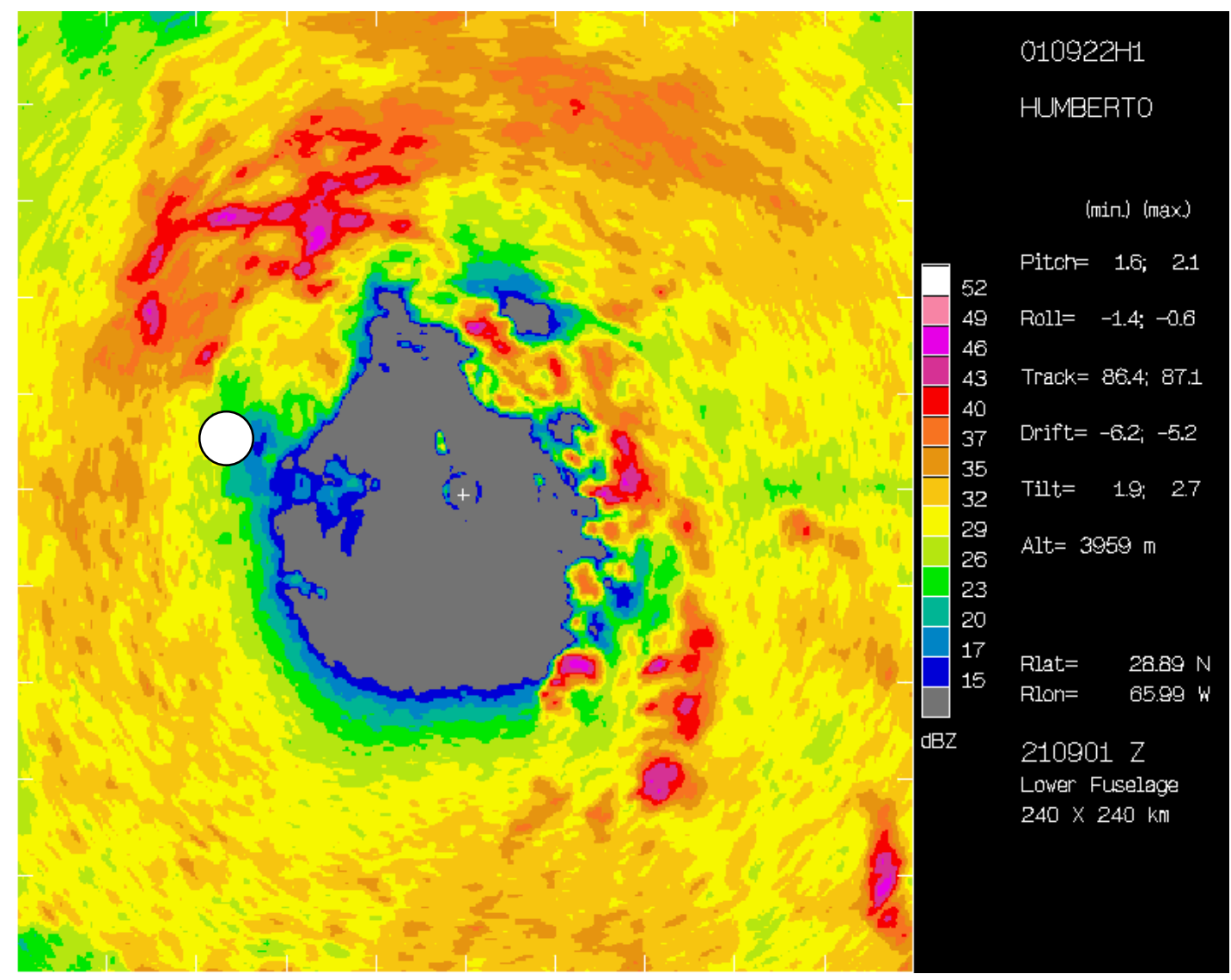

Figure 46. Plan view reflectivity image at 21:09 UTC on September $22^{\text {nd }}$. Image is 240 x $240 \mathrm{~km}$. White dot shows the center of low pressure. 


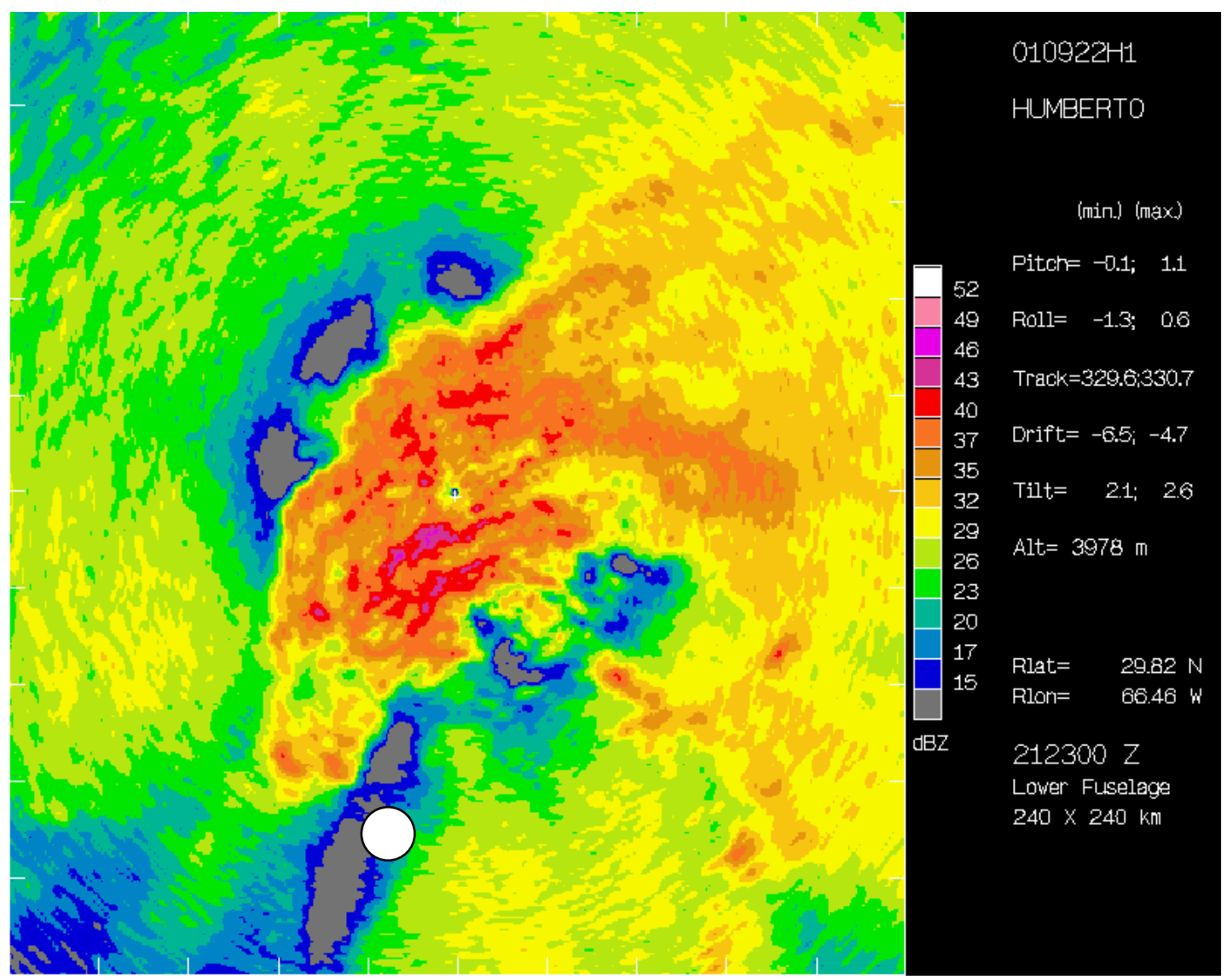

Figure 47. Plan view reflectivity image at 21:23 UTC on September $22^{\text {nd }}$. Image is $240 \mathrm{x}$ $240 \mathrm{~km}$. White dot shows the center of low pressure. 


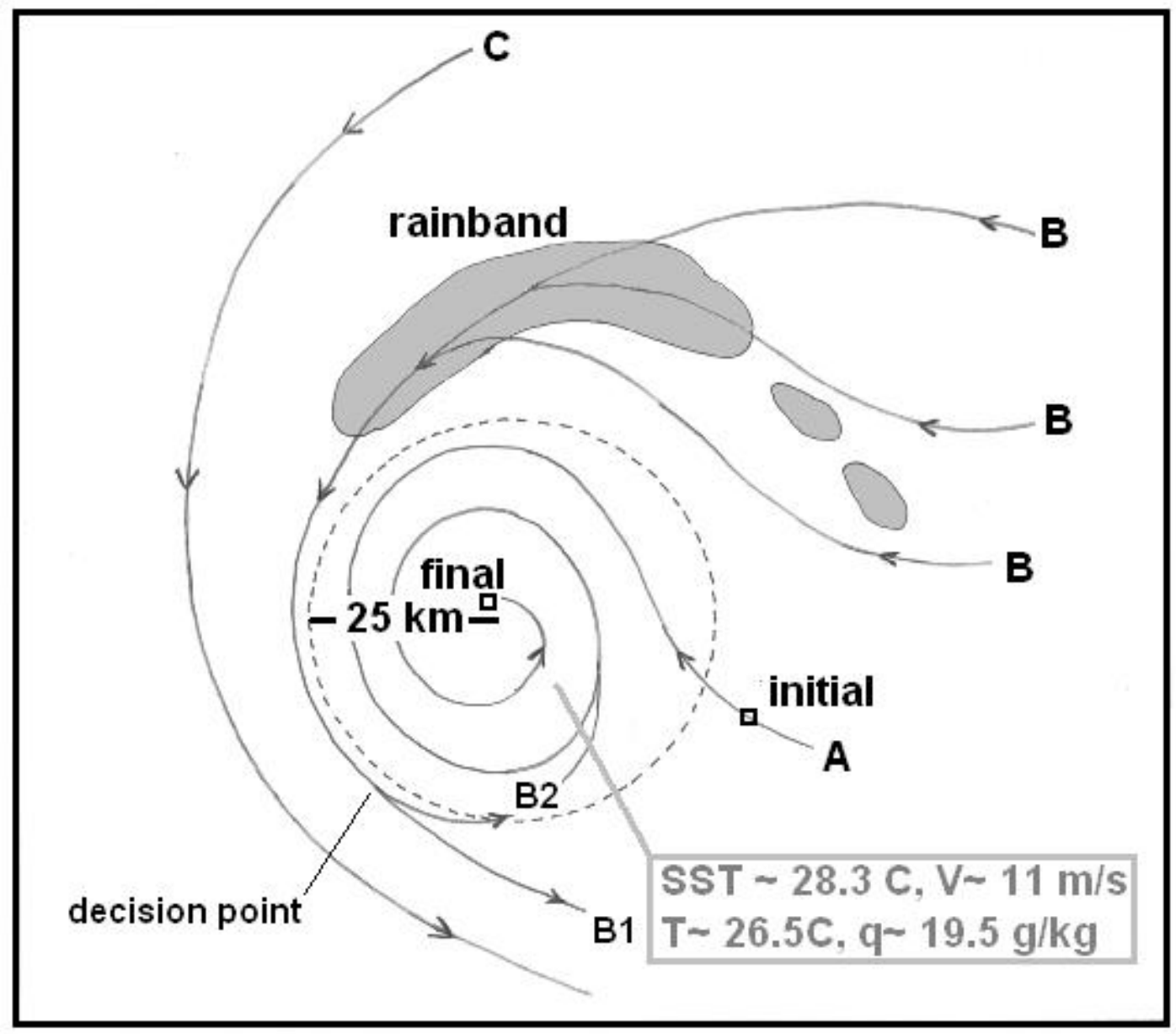

Figure 48. Schematic showing the three trajectories. The black dashed line depicts the 25 $\mathrm{km}$ radius from the center. "A", " $\mathrm{B}$ ", and " $\mathrm{C}$ " mark the 3 trajectories. The point marked "decision point" displays where trajectory "B" splits. "B2" is the first trajectory analyzed. "B1" displays the new trajectory based on the results of the first energy budget. 


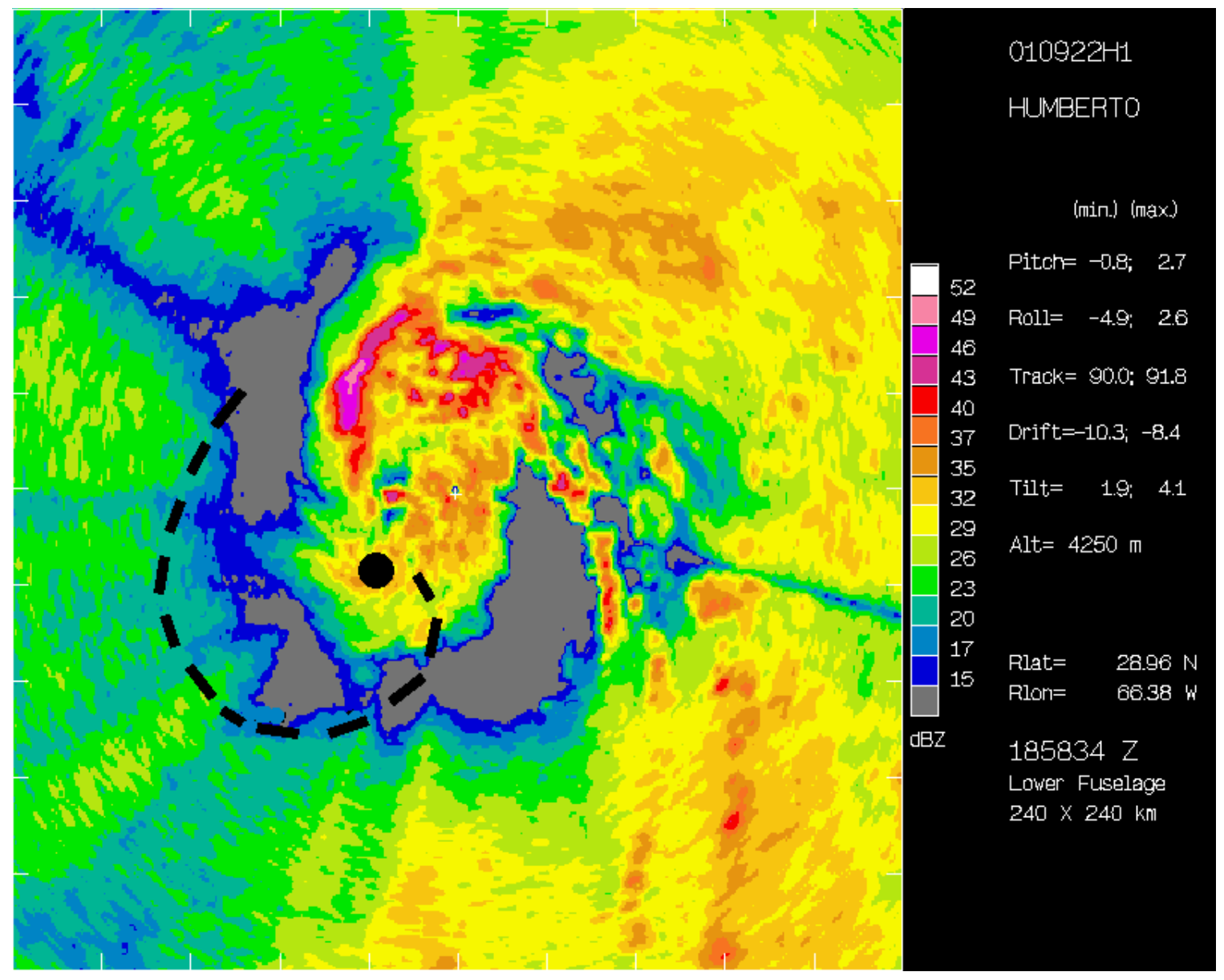

Figure 49. Plan view reflectivity image at 18:58 UTC on September $22^{\text {nd }}$. Image is $240 \mathrm{x}$ $240 \mathrm{~km}$. Black dot shows the center of lowest surface pressure and black dashed line indicates the inflow trajectory. 


\section{0 m Equivalent Potential Temperature (K)}

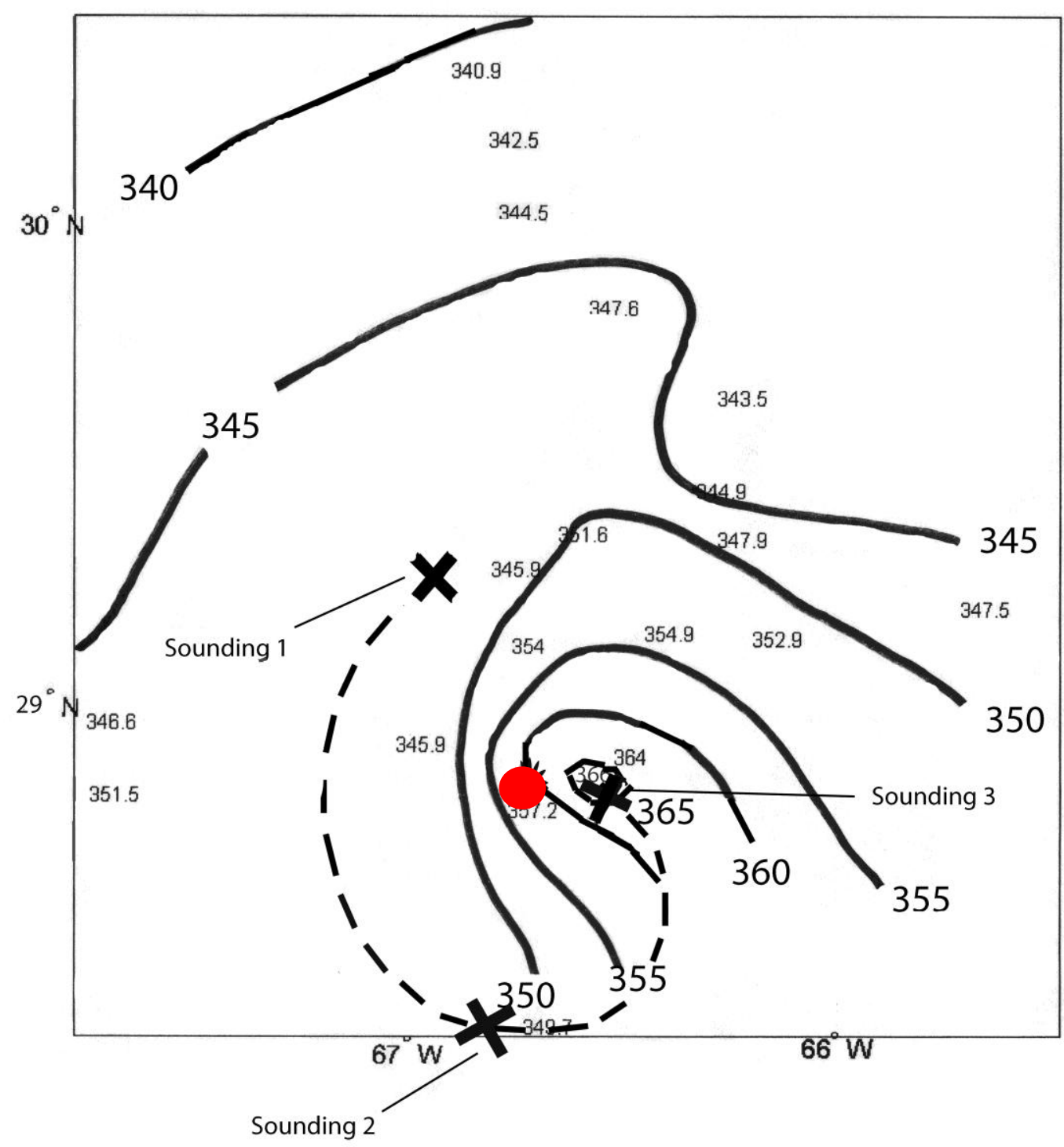

Figure 50. Plan view of $\theta_{\mathrm{e}}$ at 10 meters. Solid contours denote equivalent potential temperature every $5 \mathrm{~K}$. Inflow trajectory is the black dashed line and the black " $\mathrm{X}$ " marks the positions of the 3 soundings. Red spot is the low-level circulation center. 


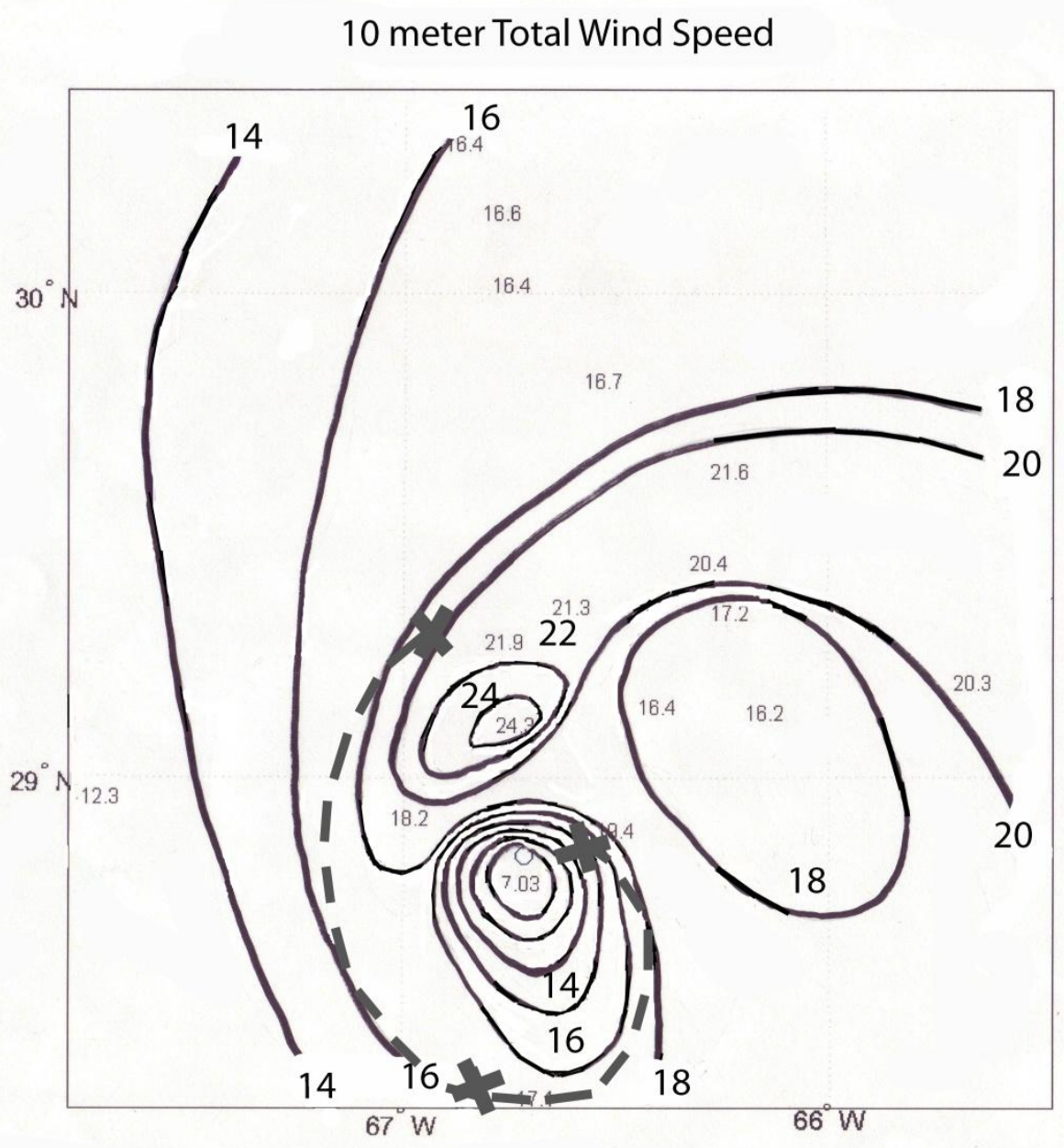

Figure 51. 10 meter plan view of earth relative total wind speed. Contours denote wind speed every $2 \mathrm{~m} \mathrm{~s}^{-1}$. Inflow trajectory is the black dashed line and the black " $\mathrm{X}$ " marks the positions of the 3 soundings. Black circle is the low-level circulation center. 


\section{$10 \mathrm{~m}$ Temperature (Celsius)}

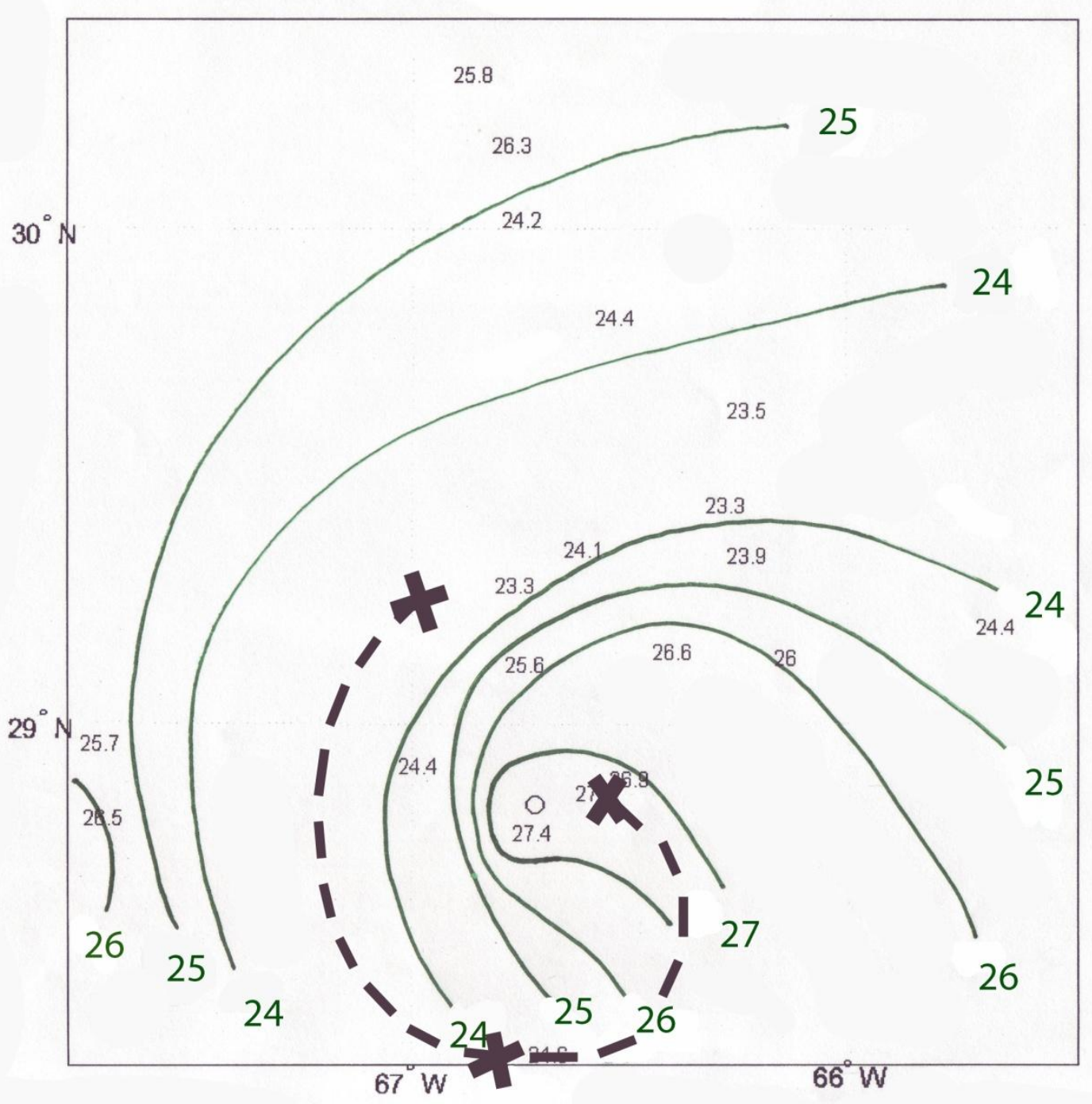

Figure 52. Plan view of the 10 meter temperature in degrees Celsius. Contours denote temperature every $1^{\circ} \mathrm{C}$. Inflow trajectory is the black dashed line and the black " $\mathrm{X}$ " marks the positions of the 3 soundings. Black circle is the low-level circulation center. 
10 m Specific Humidity $(\mathrm{g} / \mathrm{kg})$

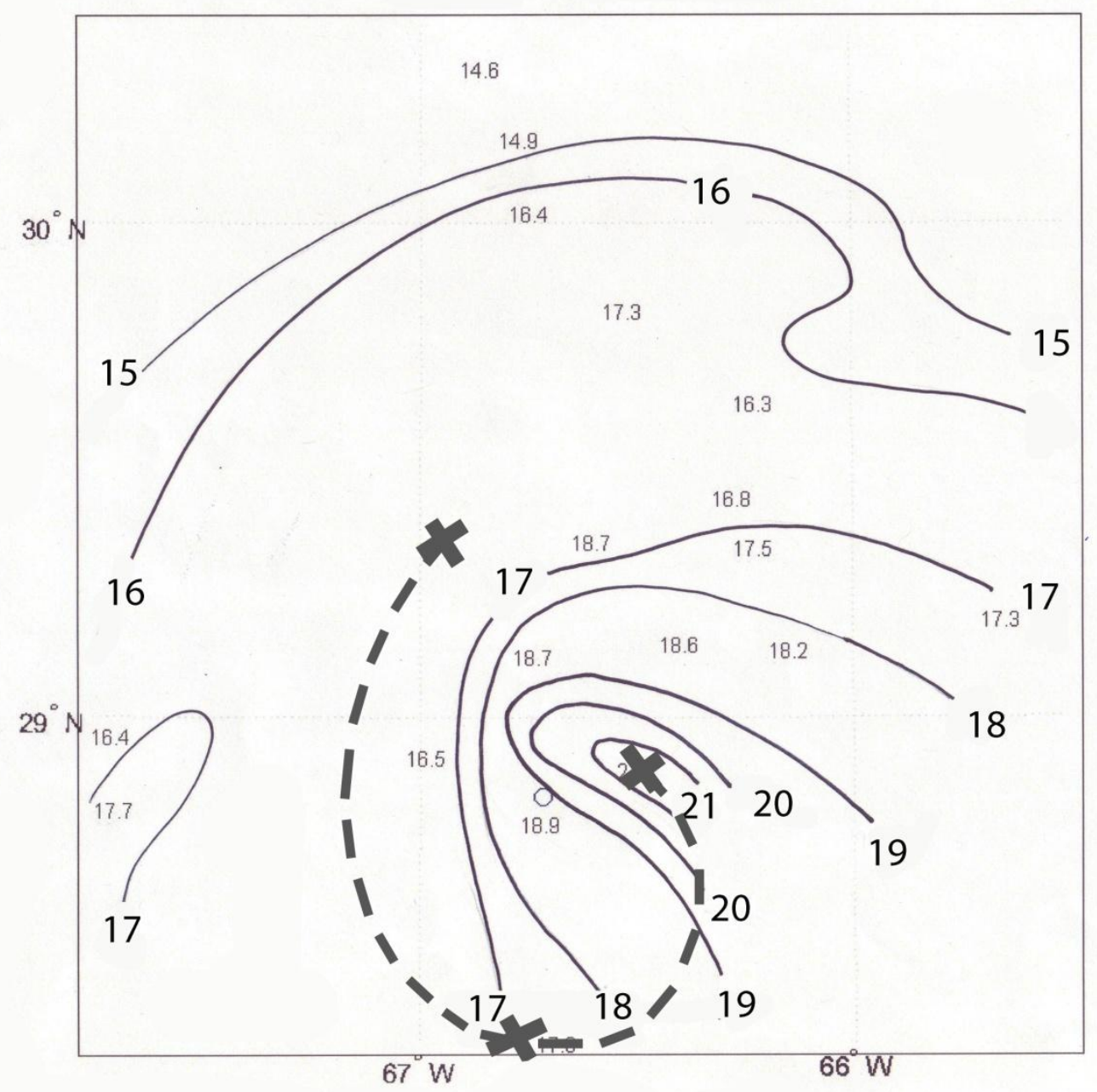

Figure 53. Plan view of the 10 meter specific humidity in $\mathrm{g} \mathrm{kg}^{-1}$. Contours denote specific humidity every $1 \mathrm{~g} \mathrm{~kg}^{-1}$. Inflow trajectory is the black dashed line and the black " $\mathrm{X}$ " marks the positions of the 3 soundings. Black circle is the low-level circulation center. 


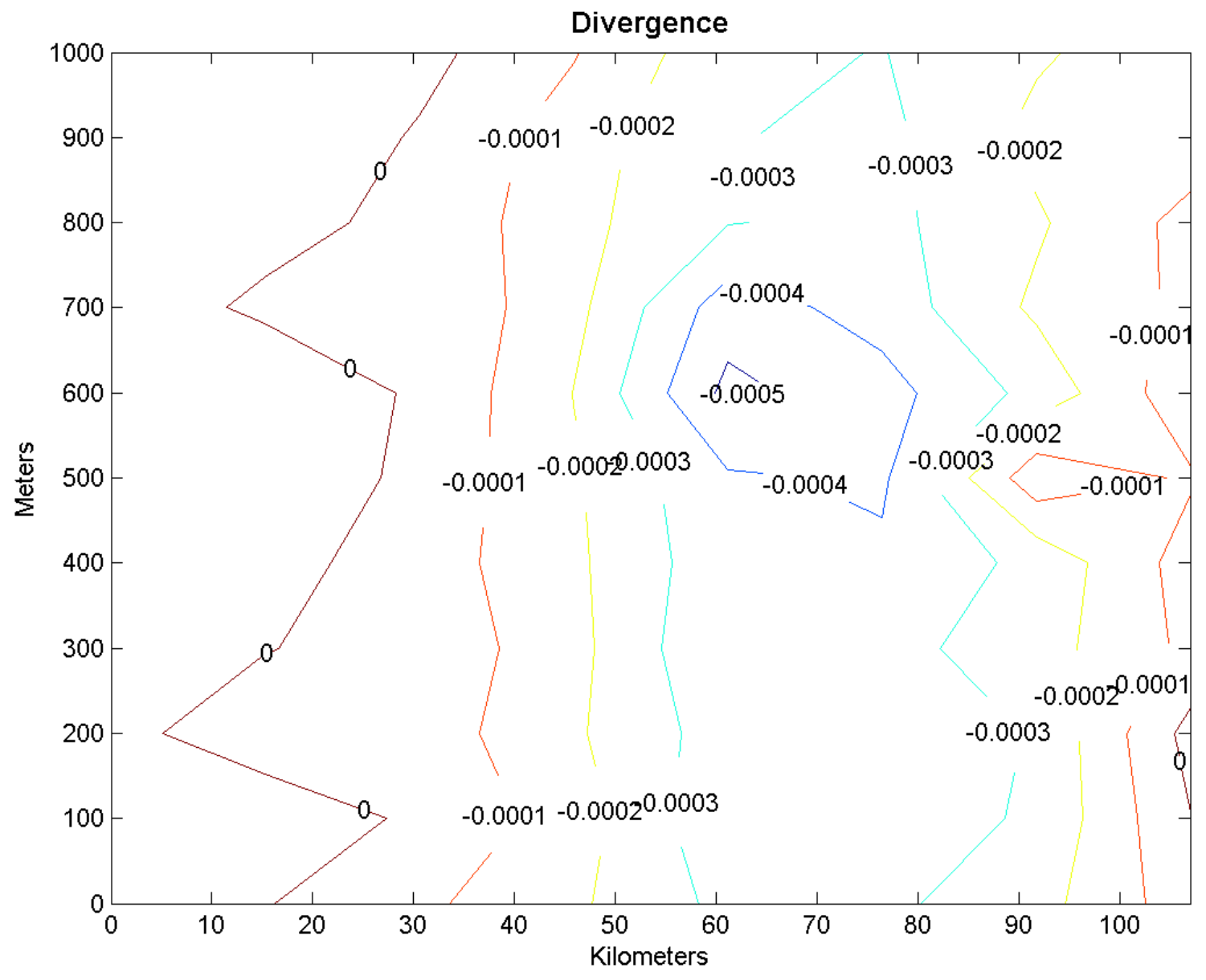

Figure 54. Vertical cross-section of divergence $\left(\mathrm{s}^{-1}\right)$ calculated from sounding 1 to 2 (refer to Fig. 50). 


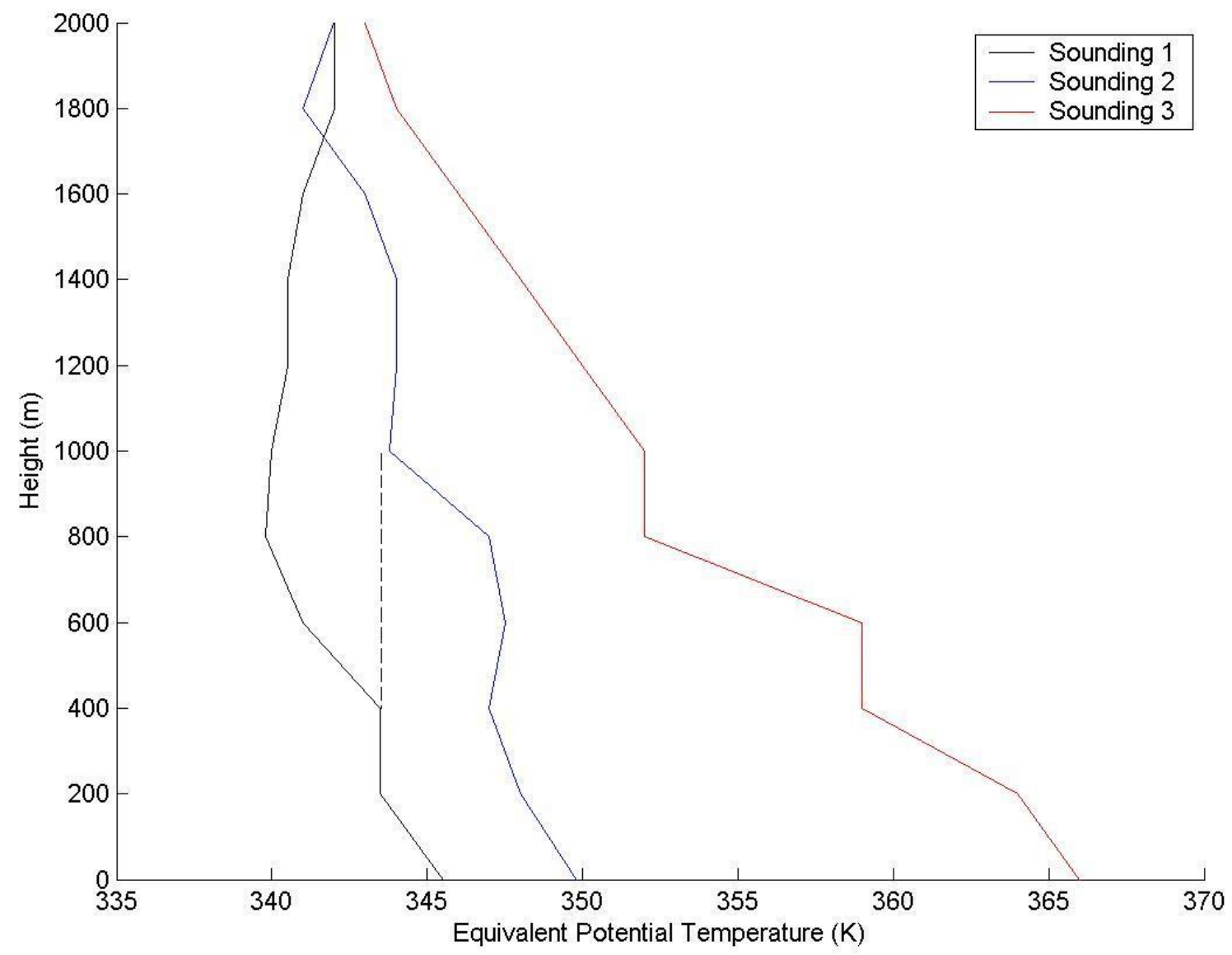

Figure 55. Vertical profiles of $\theta_{\mathrm{e}}$ soundings along the inflow path "B2". Sounding 1, 2, and 3 are from Fig. 50. Dashed line displays how the mixed layer rises in the presence of convergence. 


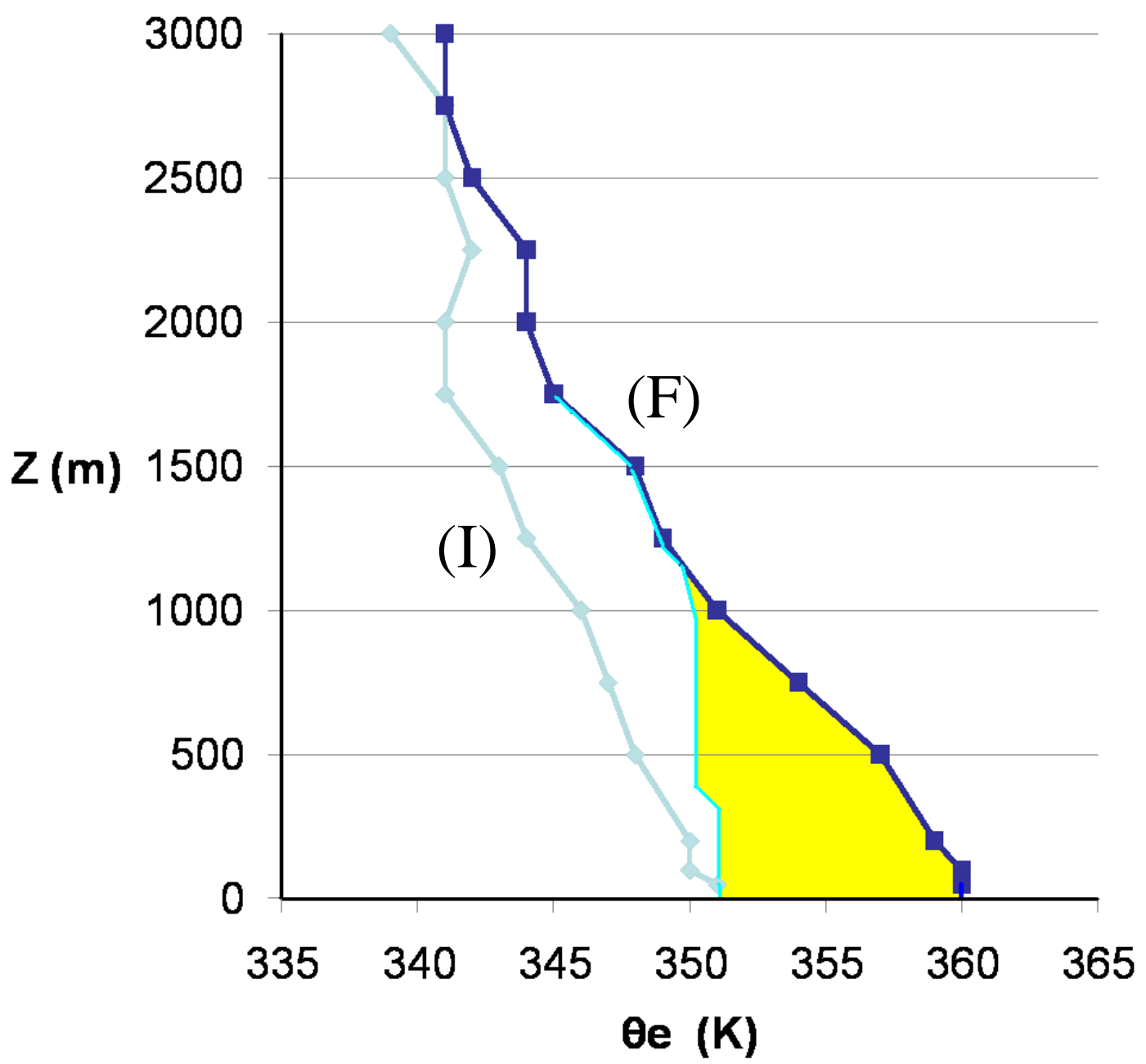

Figure 56. The initial sounding marked "I" and final sounding marked "F" for trajectory "A" shown in Fig. 48. Yellow displays amount of $\theta_{\mathrm{e}}$ gained with the divergence adjusted sounding. 


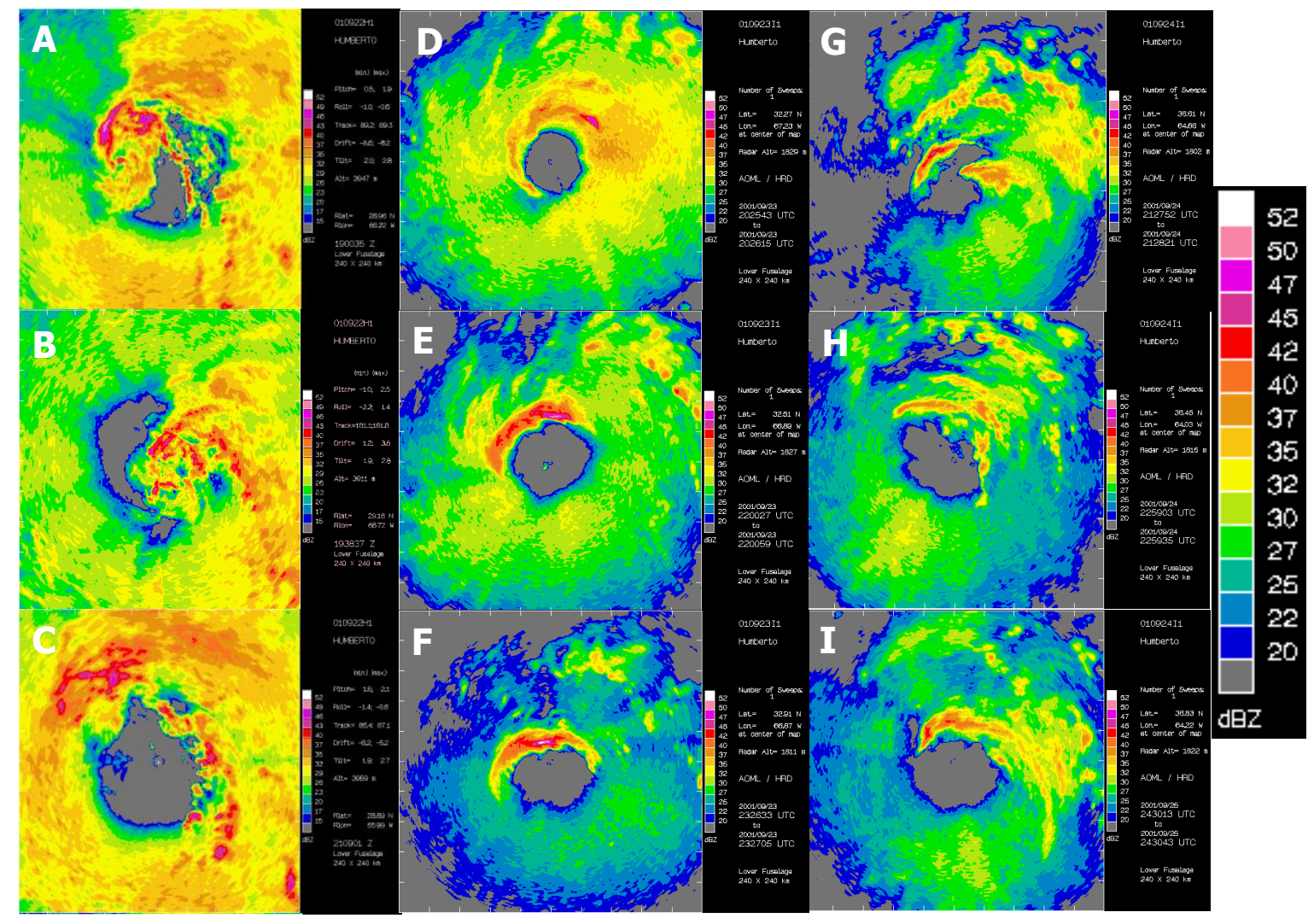

Figure 57. Nine lower fuselage scans $(240 \mathrm{~km}$ x $240 \mathrm{~km})$ of evolution of eyewall. Times to the nearest minute and dates are (A) 19:00 UTC 9/22/01 (B) 19:38 UTC 9/22/01 (C) 21:16 UTC 9/22/01 (D) 20:26 UTC 9/23/01 (E) 22:59 UTC 9/23/01 (F) 24:30 UTC 9/23/01 (G) 21:27 UTC 9/24/01 (H) 22:59 UTC 9/24/01 (I) 24:30 UTC 9/24/01. Color scale on right indicates $\mathrm{dBZ}$ values. 


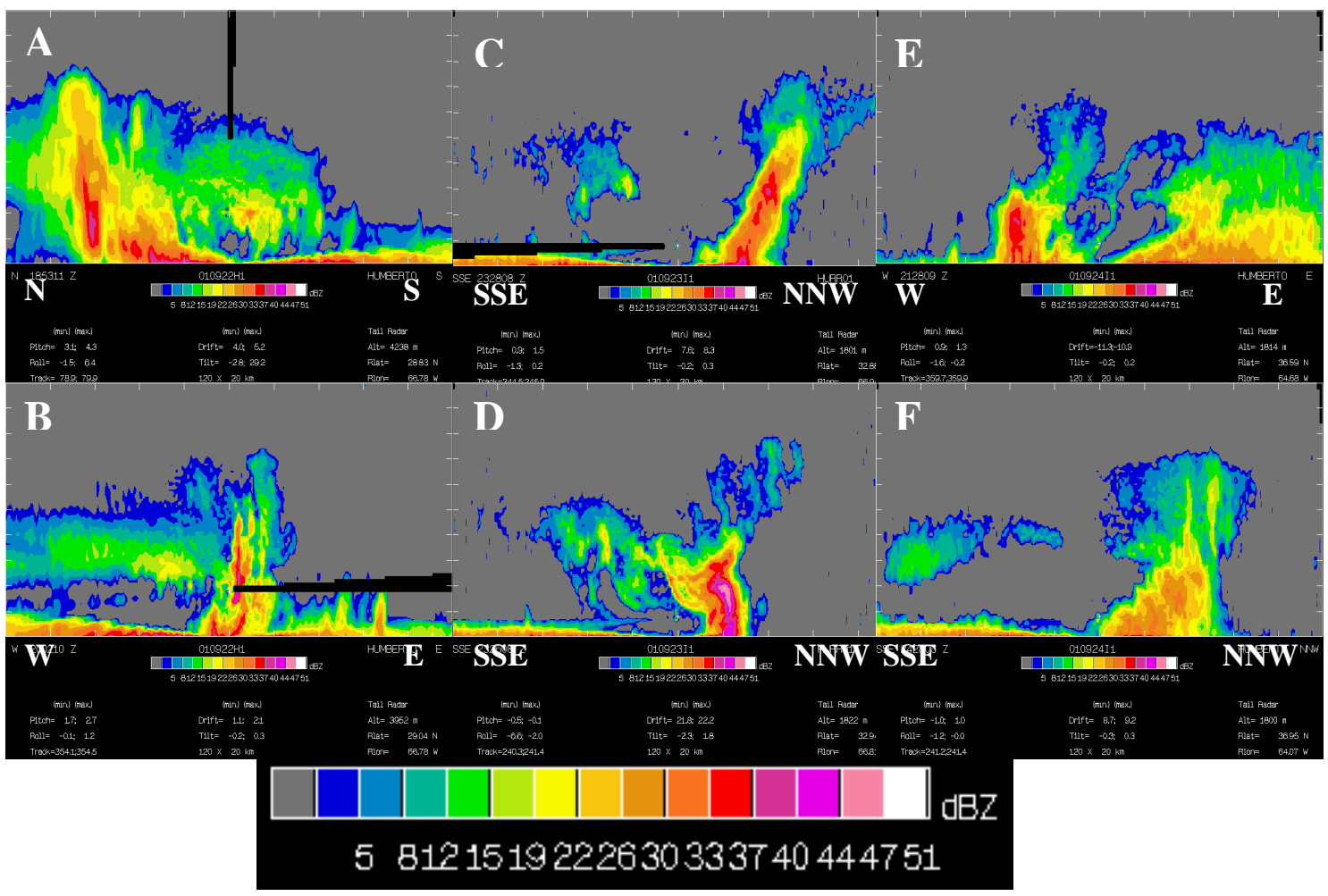

Figure 58. Six lower fuselage scans $(120 \mathrm{~km}$ x $20 \mathrm{~km})$ of evolution of eyewall. Times to the nearest minute and dates are (A) 18:53 UTC 9/22/01 (B) 20:02 UTC 9/22/01 (C) 23:28 UTC 9/23/01 (D) 23:26 UTC 9/23/01 (E) 21:28 UTC 9/24/01 (F) 24:28 UTC $9 / 24 / 01$. Color scale on bottom indicates dBZ values. 
A

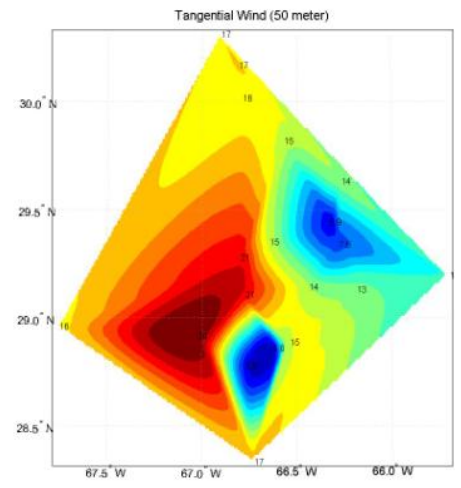

C

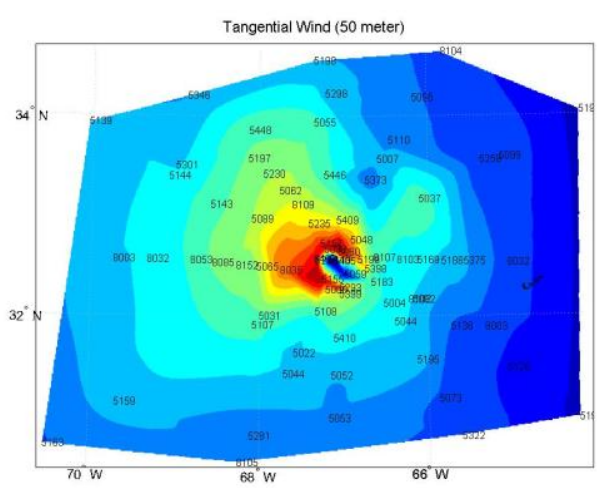

$\mathbf{E}$

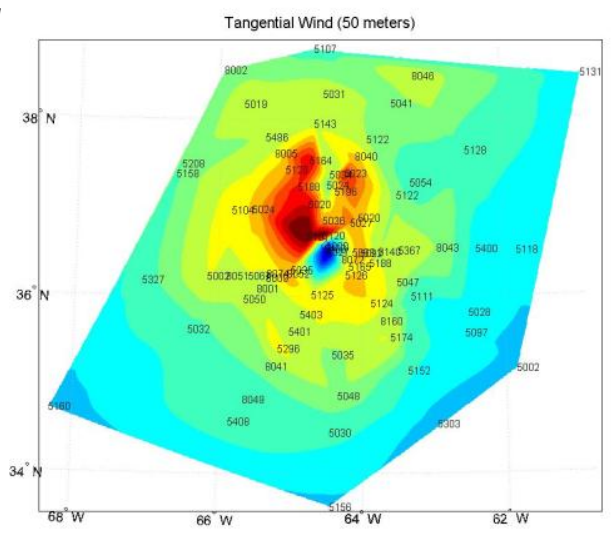

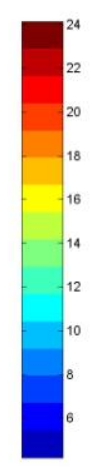
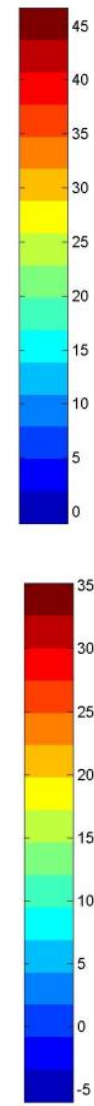

B

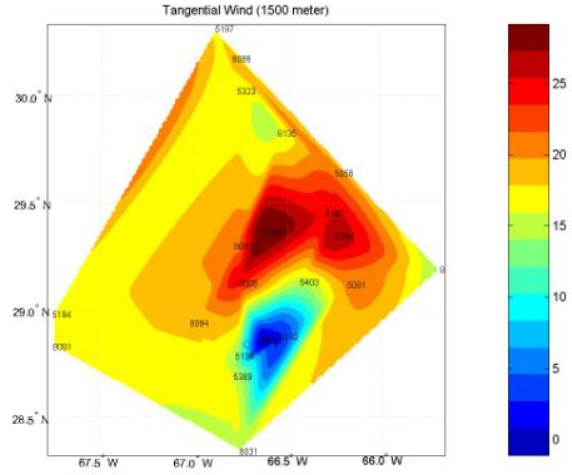

D
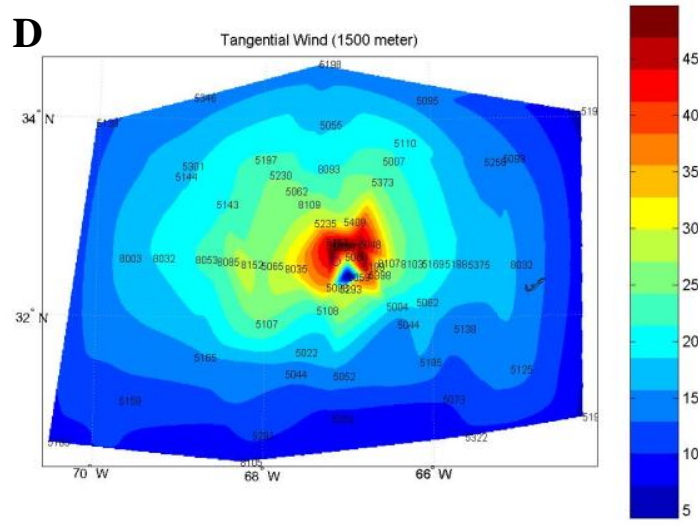

$\mathbf{F}$

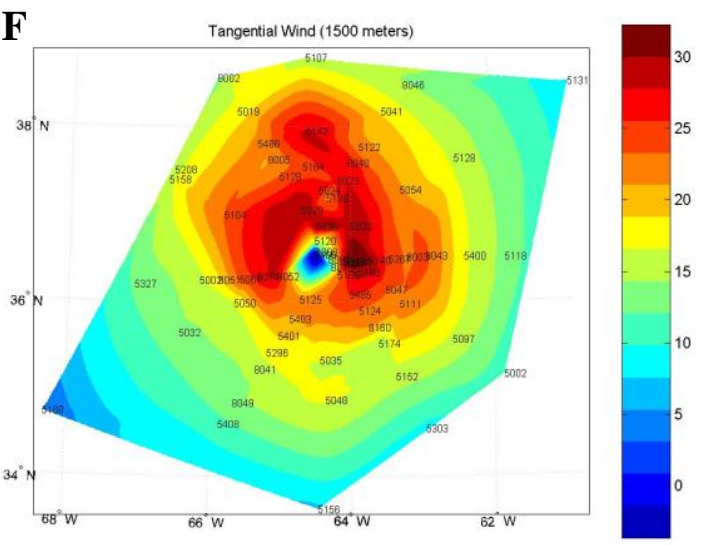

Figure 59. Plan views of the tangential winds $\left(\mathrm{m} \mathrm{s}^{-1}\right)$ at $50 \mathrm{~m}$ and $1.5 \mathrm{~km}$ for various days of the experiment. Color bar to right delineates the magnitude $\left(\mathrm{m} \mathrm{s}^{-1}\right)$. Note difference in horizontal scale for each day. (A) $50 \mathrm{~m}$ on $9 / 22 / 01$ (B) $1.5 \mathrm{~km}$ on $9 / 22 / 01$ (C) $50 \mathrm{~m}$ on $9 / 23 / 01$ (D) $1.5 \mathrm{~km}$ on $9 / 23 / 01$ (E) $50 \mathrm{~km}$ on $9 / 24 / 01$ (F) $1.5 \mathrm{~km}$ on 9/24/01. 
A

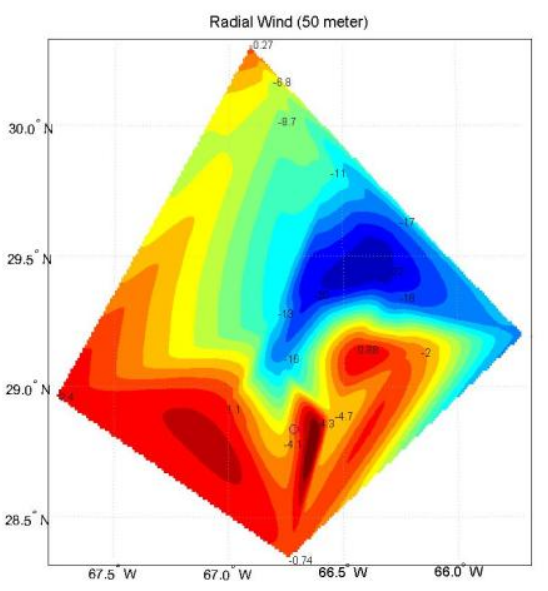

C
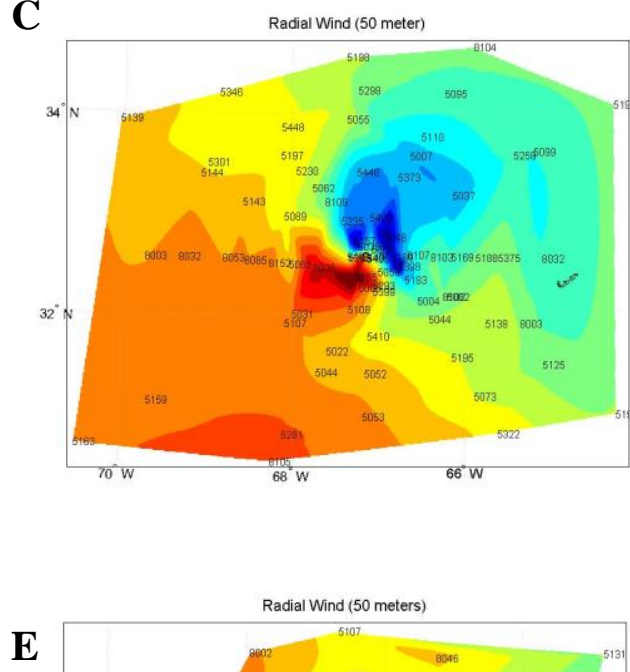

$\mathbf{E}$

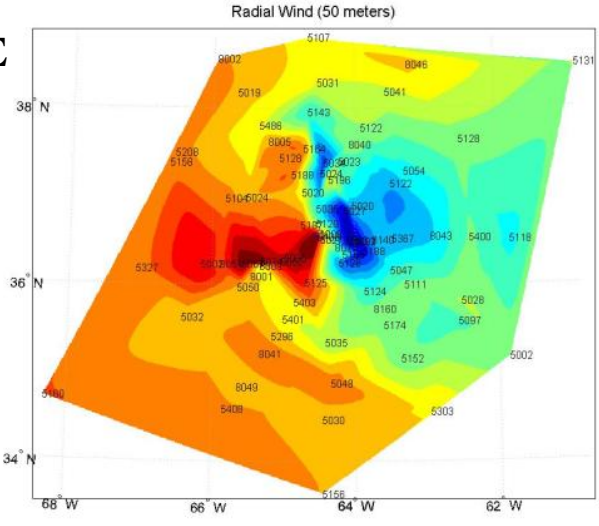

B
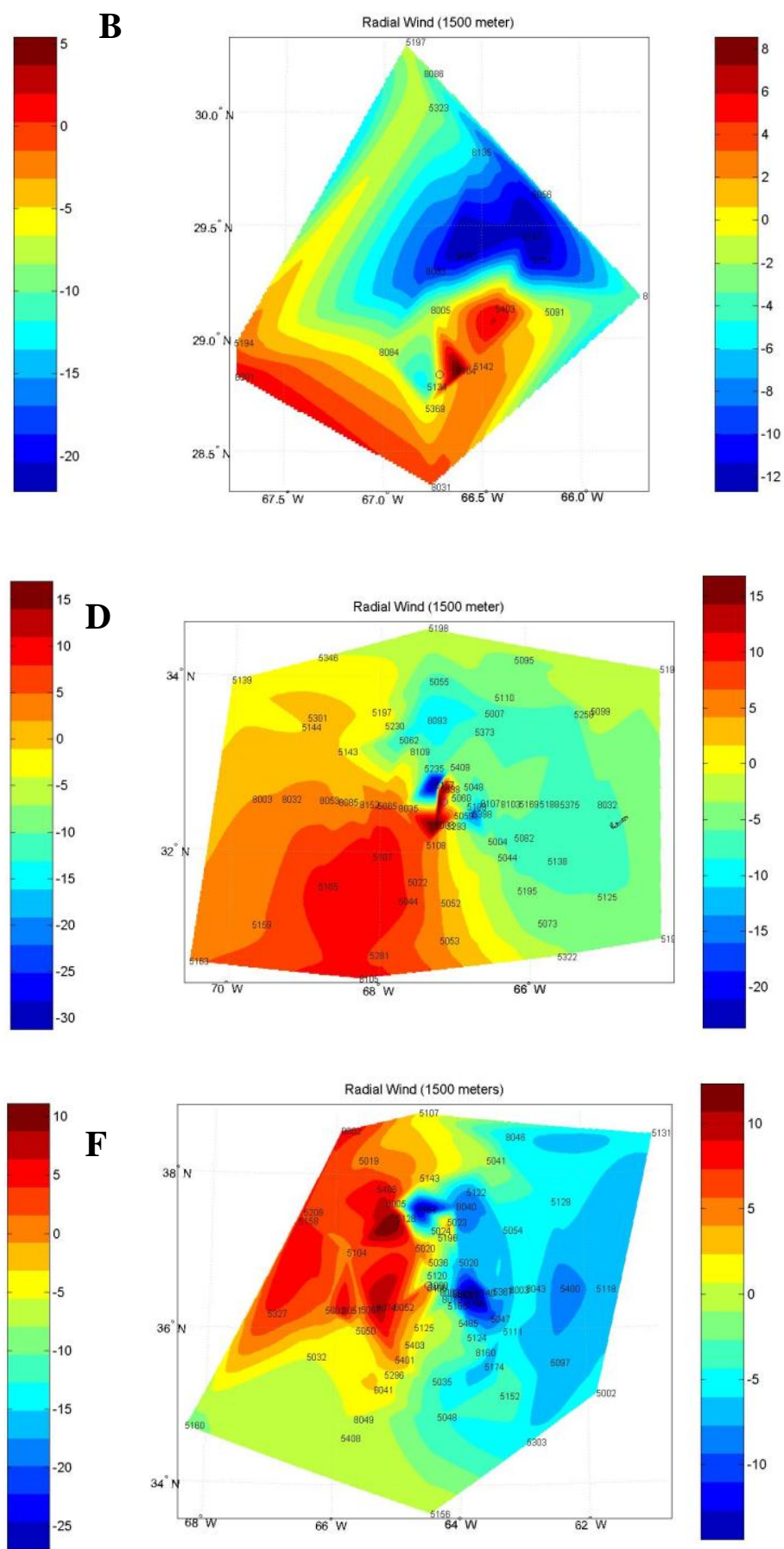

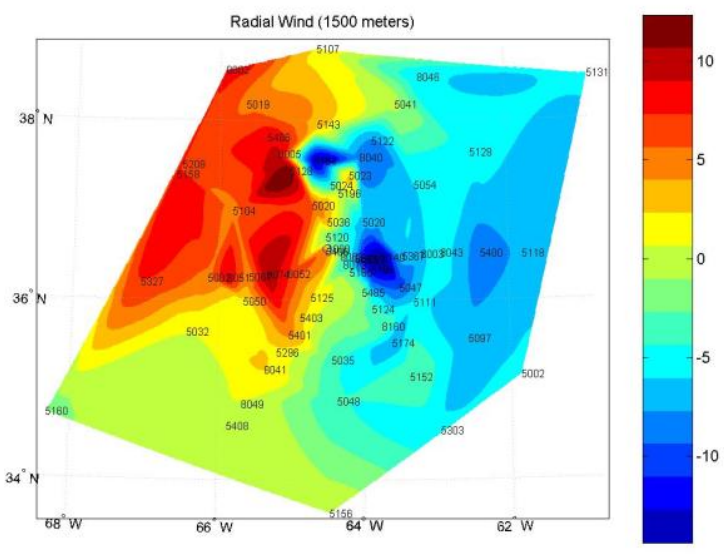

Figure 60. Plan views of the radial winds $\left(\mathrm{m} \mathrm{s}^{-1}\right)$ at $50 \mathrm{~m}$ and $1.5 \mathrm{~km}$ for various days of the experiment. Color bar to right delineates the magnitude $\left(\mathrm{m} \mathrm{s}^{-1}\right)$. Note difference in horizontal scale for each day. (A) $50 \mathrm{~m}$ on $9 / 22 / 01$ (B) $1.5 \mathrm{~km}$ on 9/22/01 (C) $50 \mathrm{~m}$ on 9/23/01 (D) $1.5 \mathrm{~km}$ on 9/23/01 (E) $50 \mathrm{~km}$ on 9/24/01 (F) $1.5 \mathrm{~km}$ on 9/24/01. 


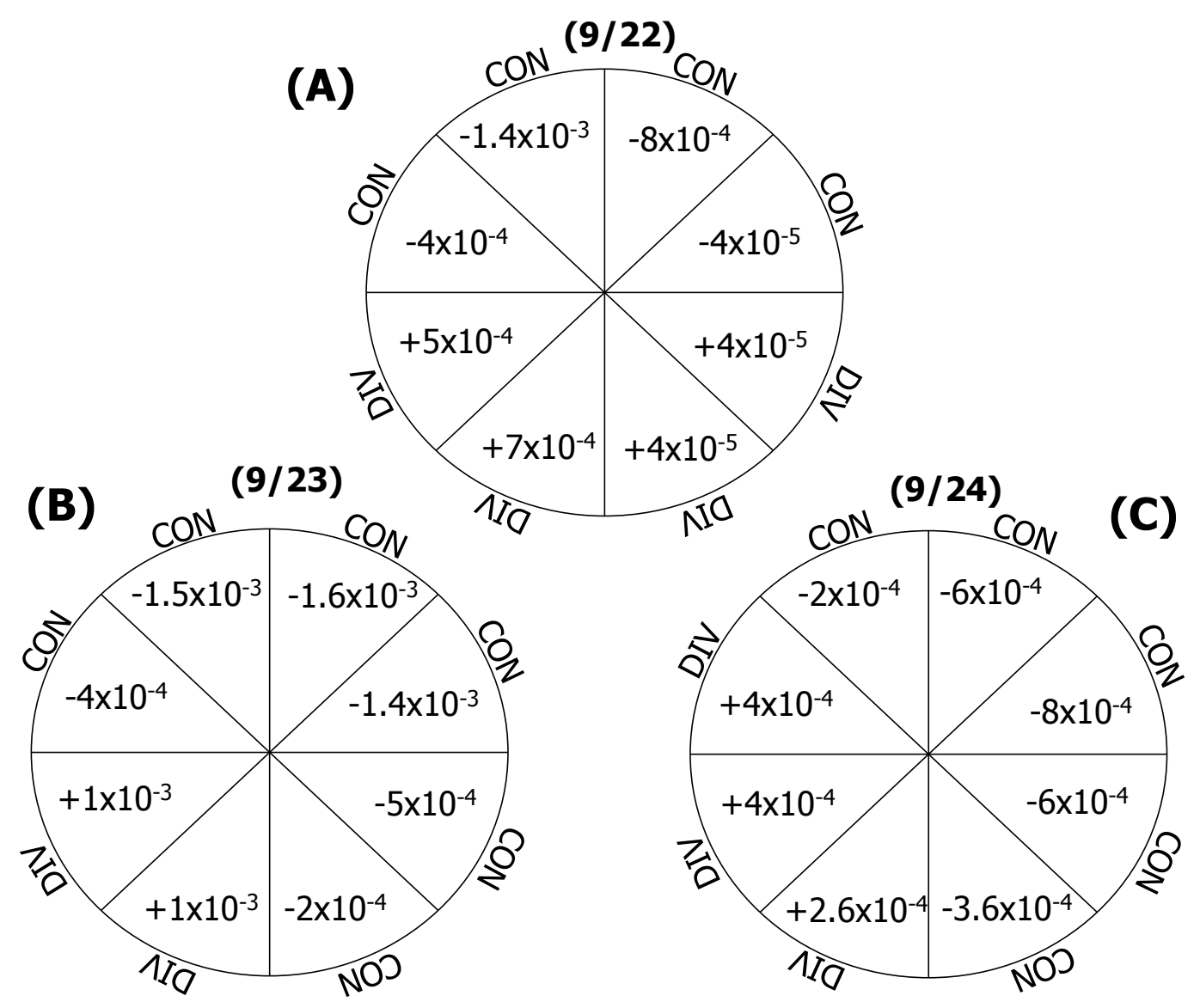

Figure 61. Divergence $\left(\mathrm{s}^{-1}\right)$ calculated at $200 \mathrm{~m}$ from just outside the eyewall to the circulation center. Circle size is a function of the eyewall radius. (A) 9/22/01 (B) 9/23/01 (C) 9/24/01. 

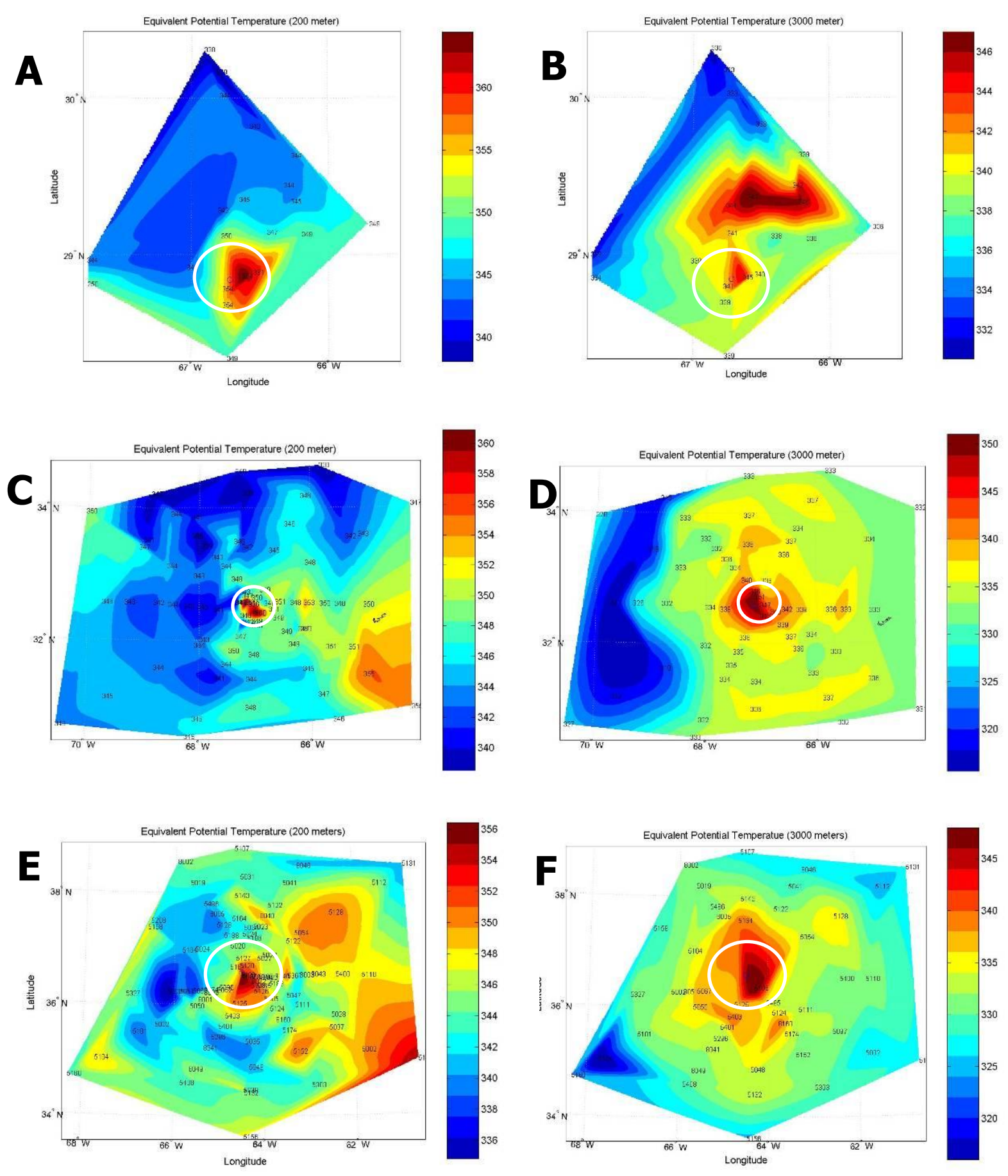

Figure 62. Plan views of $\theta_{\mathrm{e}}$ for various days of the experiment at $200 \mathrm{~m}$ and $3 \mathrm{~km}$. Color bar to right delineates the magnitude (Kelvin). Note difference in horizontal scale for each day. (A) $200 \mathrm{~m}$ on $9 / 22 / 01$ (B) $3 \mathrm{~km}$ on $9 / 22 / 01$ (C) $200 \mathrm{~m}$ on 9/23/01 (D) $3 \mathrm{~km}$ on $9 / 23 / 01$ (E) $200 \mathrm{~km}$ on $9 / 24 / 01$ (F) $3 \mathrm{~km}$ on 9/24/01. The white rings show the location of the eyewall based on the reflectivity. 

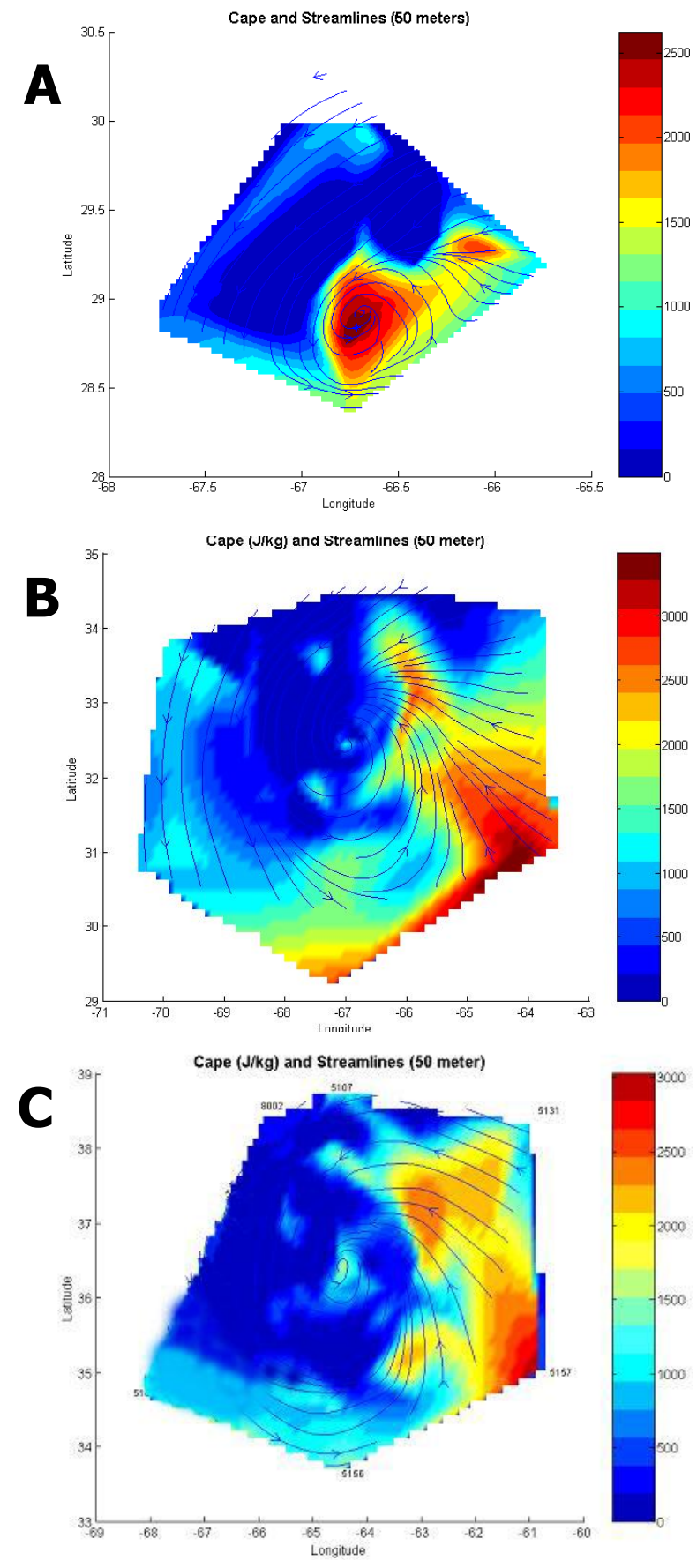

Figure 63. Large-scale plan view of CAPE and streamlines at 50 meters. Color bar shows values of CAPE in $\mathrm{J} \mathrm{kg}^{-1}$. Note difference in horizontal scales for each day. (A) 9/22/01 (B) 9/23/01 (C) 9/23/01. 

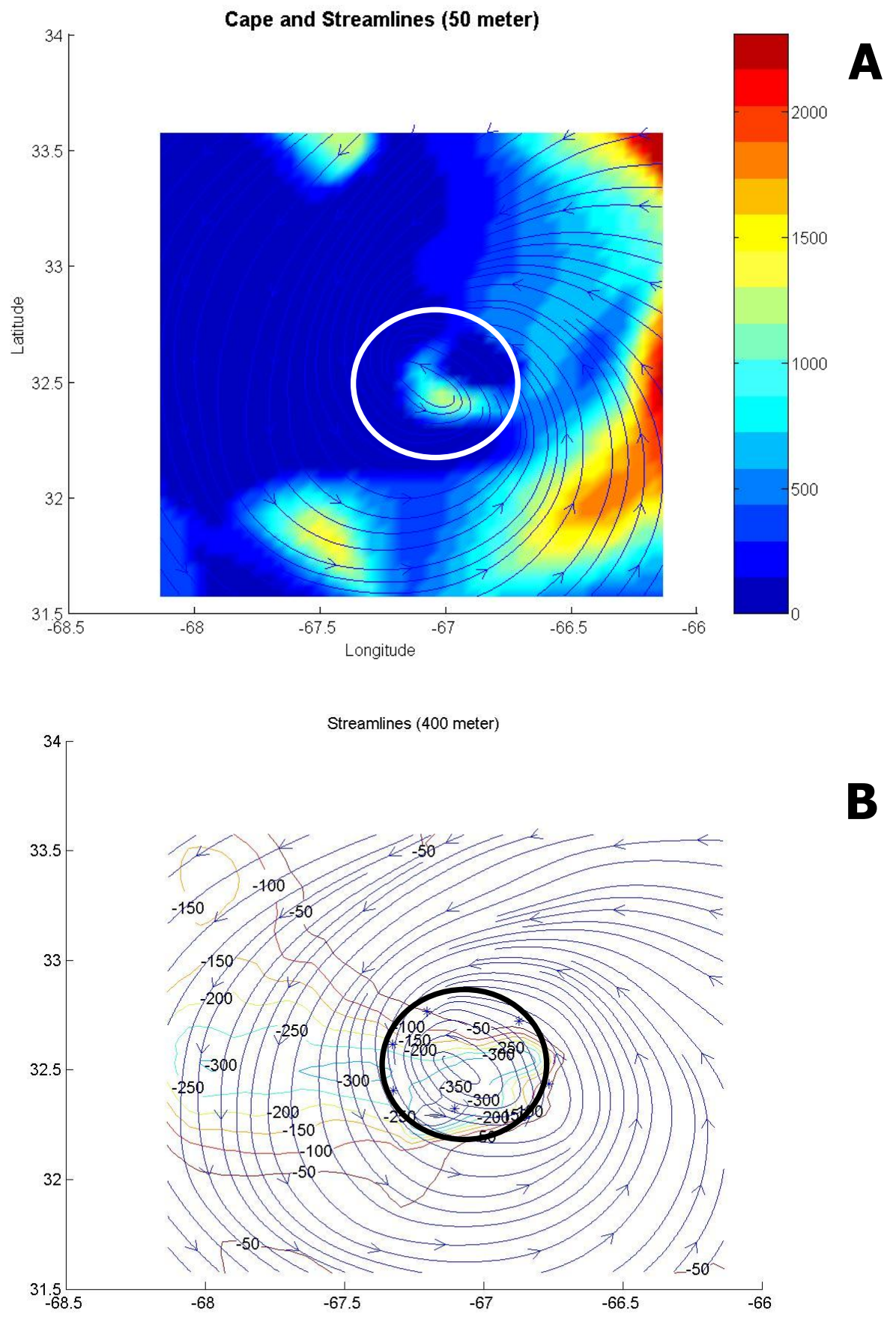

B

Figure $64.200 \mathrm{~km}$ x $200 \mathrm{~km}$ plan views of convective available potential energy $\left(\mathrm{J} \mathrm{kg}^{-1}\right)$ (CAPE), convective inhibition $\left(\mathrm{J} \mathrm{kg}^{-1}\right)(\mathrm{CIN})$, and streamlines. Latitude and longitude delineated every 0.5 degrees. White and black circles are approximate location of the RMW from the low-level WP-3D (A) CAPE on 9/23/01 (B) CIN on 9/23/01. 

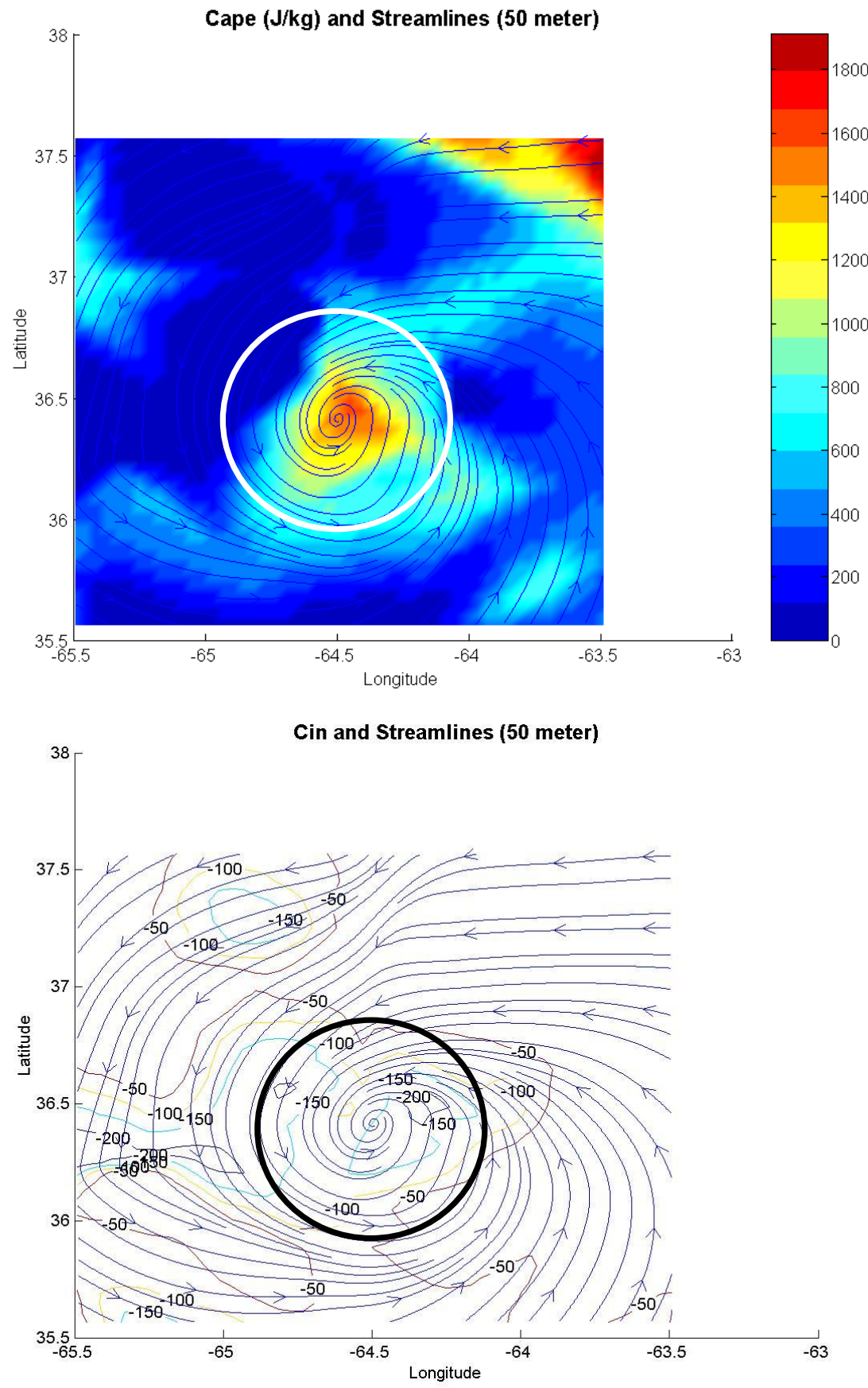

B

Figure $65.200 \mathrm{~km}$ x $200 \mathrm{~km}$ plan views of convective available potential energy $\left(\mathrm{J} \mathrm{kg}^{-1}\right)$ (CAPE), convective inhibition $\left(\mathrm{J} \mathrm{kg}^{-1}\right)(\mathrm{CIN})$, and streamlines. Latitude and longitude delineated every 0.5 degrees. White and black circles are approximate location of the RMW from the low-level WP-3D (A) CAPE on 9/24/01 (B) CIN on 9/24/01. 

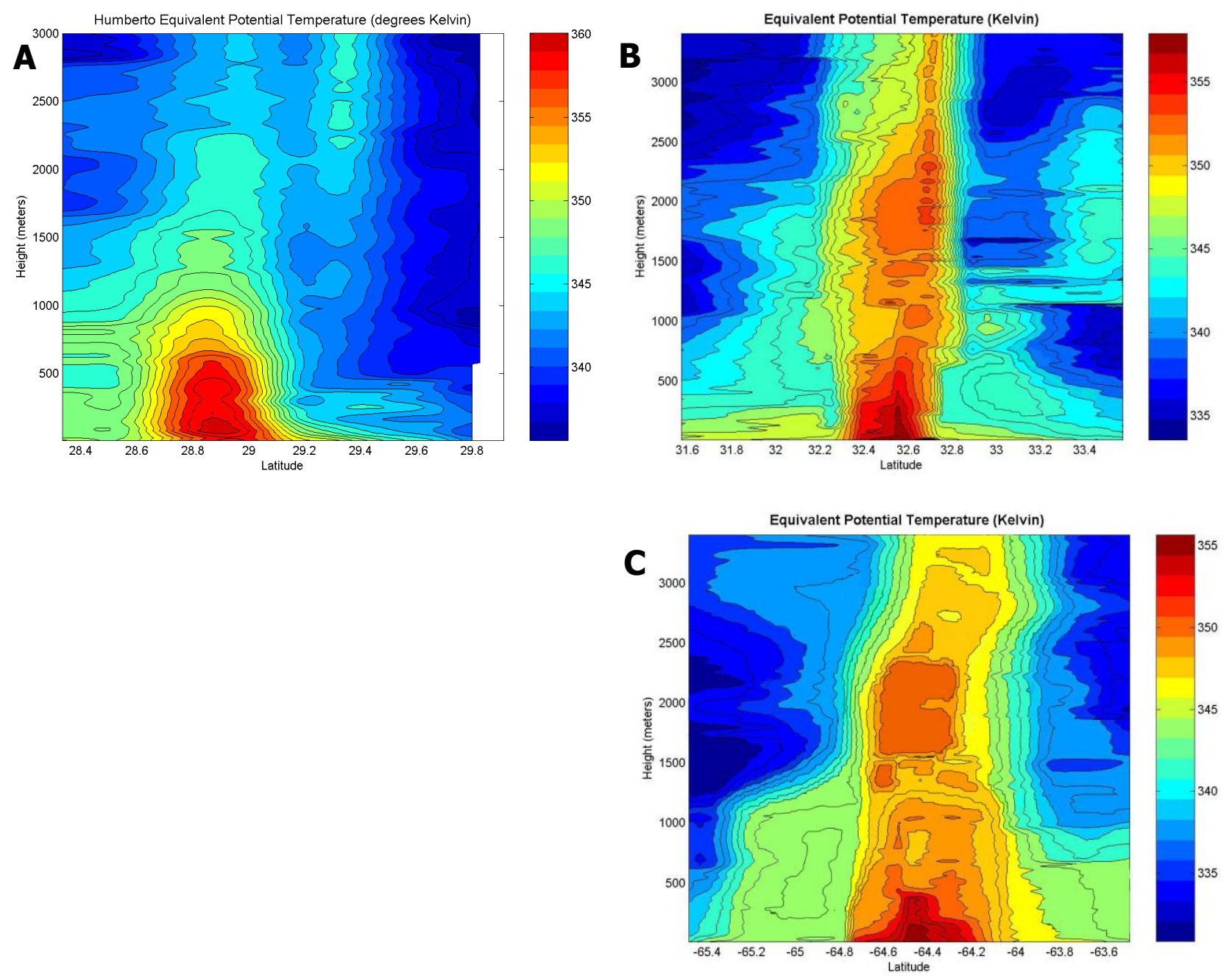

Figure 66. Vertical cross-sections of $\theta_{\mathrm{e}}$ (Kelvin). (A) $160 \mathrm{~km}$ x $3 \mathrm{~km}$ on 9/22/01.

Southern eyewall is located at $28.6^{\circ} \mathrm{N}$. Northern eyewall is located at $29.1^{\circ} \mathrm{N}$ (B) 200 $\mathrm{km} \times 3.4 \mathrm{~km}$ on $9 / 23 / 01$. Southern eyewall is located at $32.3^{\circ} \mathrm{N}$. Northern eyewall is located at $32.8^{\circ}$ N. (C) $200 \mathrm{~km} \mathrm{x} 3.4 \mathrm{~km}$ on $9 / 24 / 01$. 
A

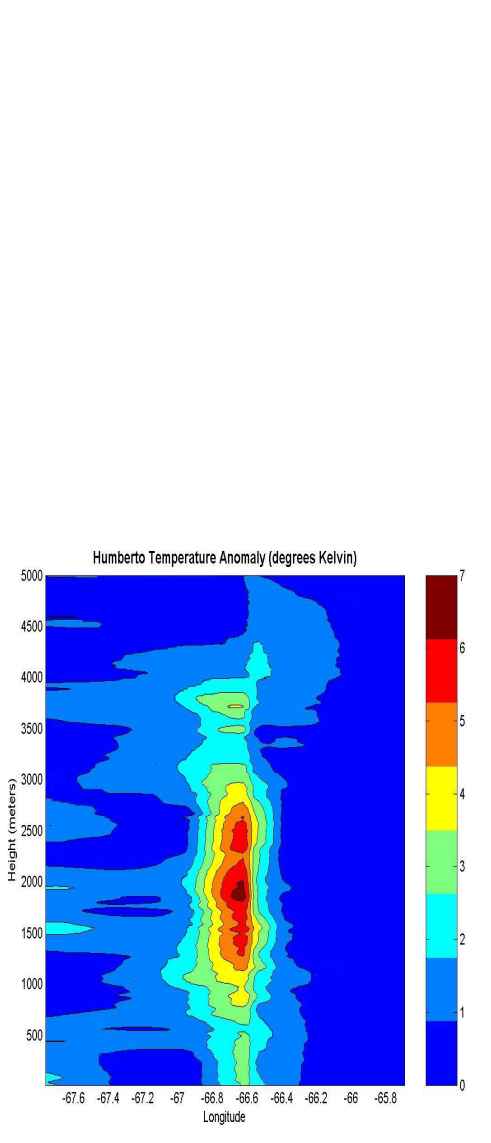

B

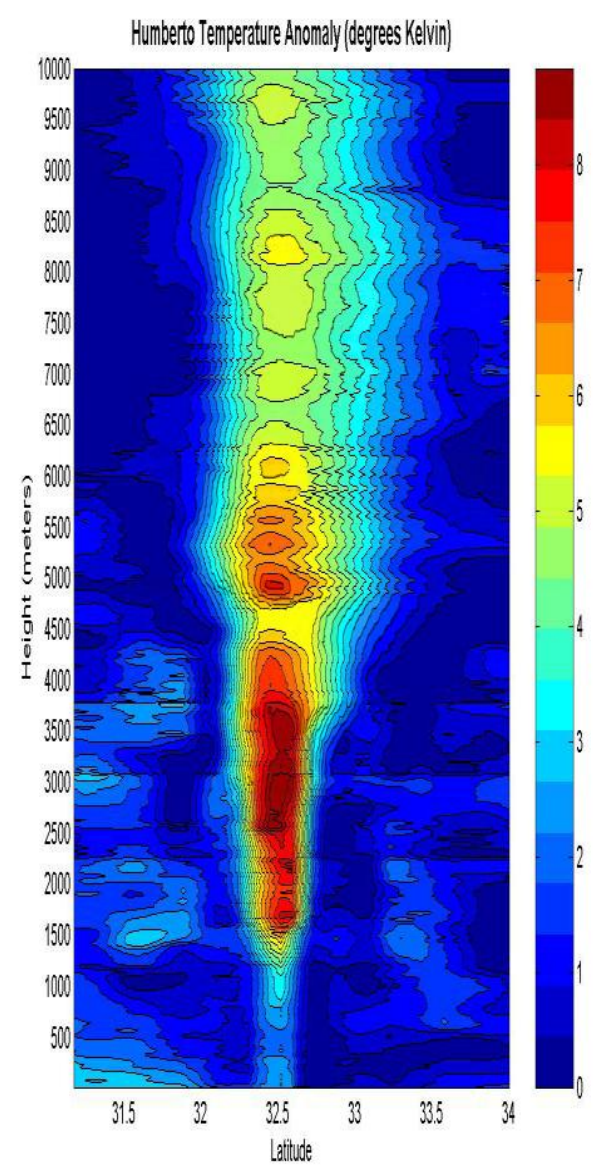

C

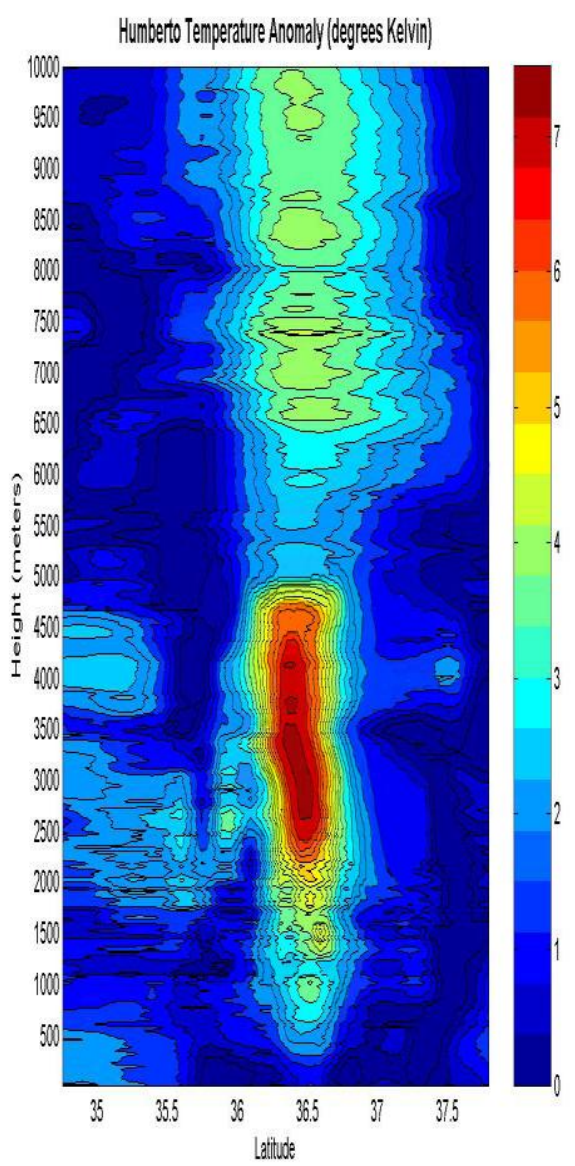

Figure 67. Vertical cross-sections of the warm core anomaly of Humberto in degrees Kelvin for (A) 9/22/01 (B) 9/23/01 (C) 9/24/01. 


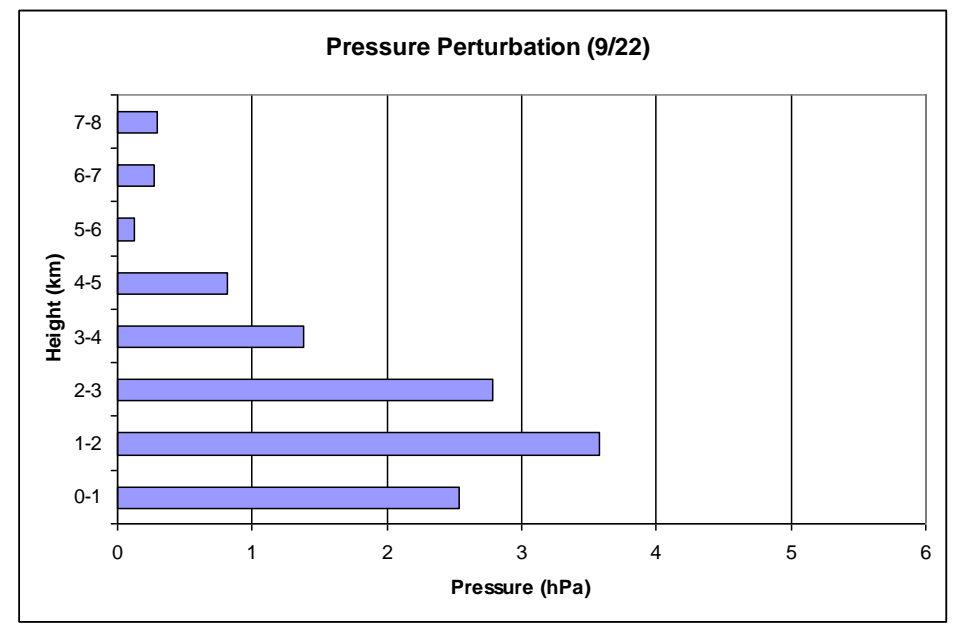

A

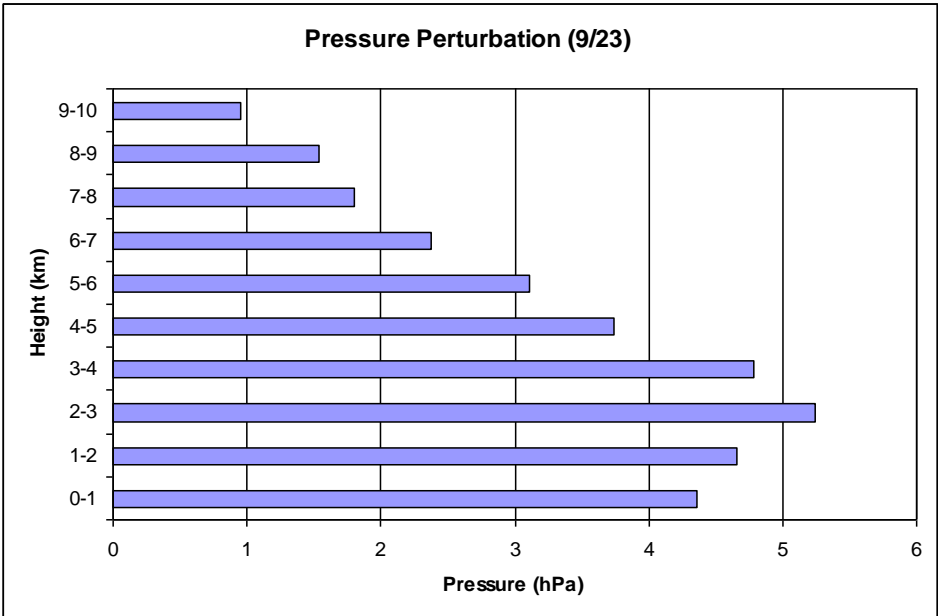

B

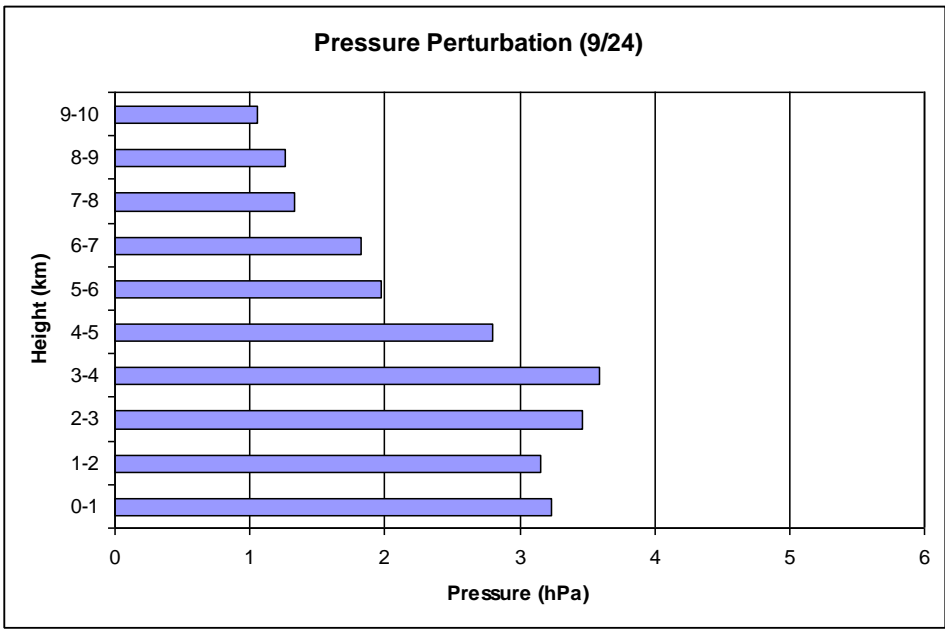

C

Figure 68. Pressure perturbation in $\mathrm{hPa}$ for $1 \mathrm{~km}$ layers from 0 to $10 \mathrm{~km}$. (A) 9/22/01 (B) 9/23/01 (C) 9/24/01. 


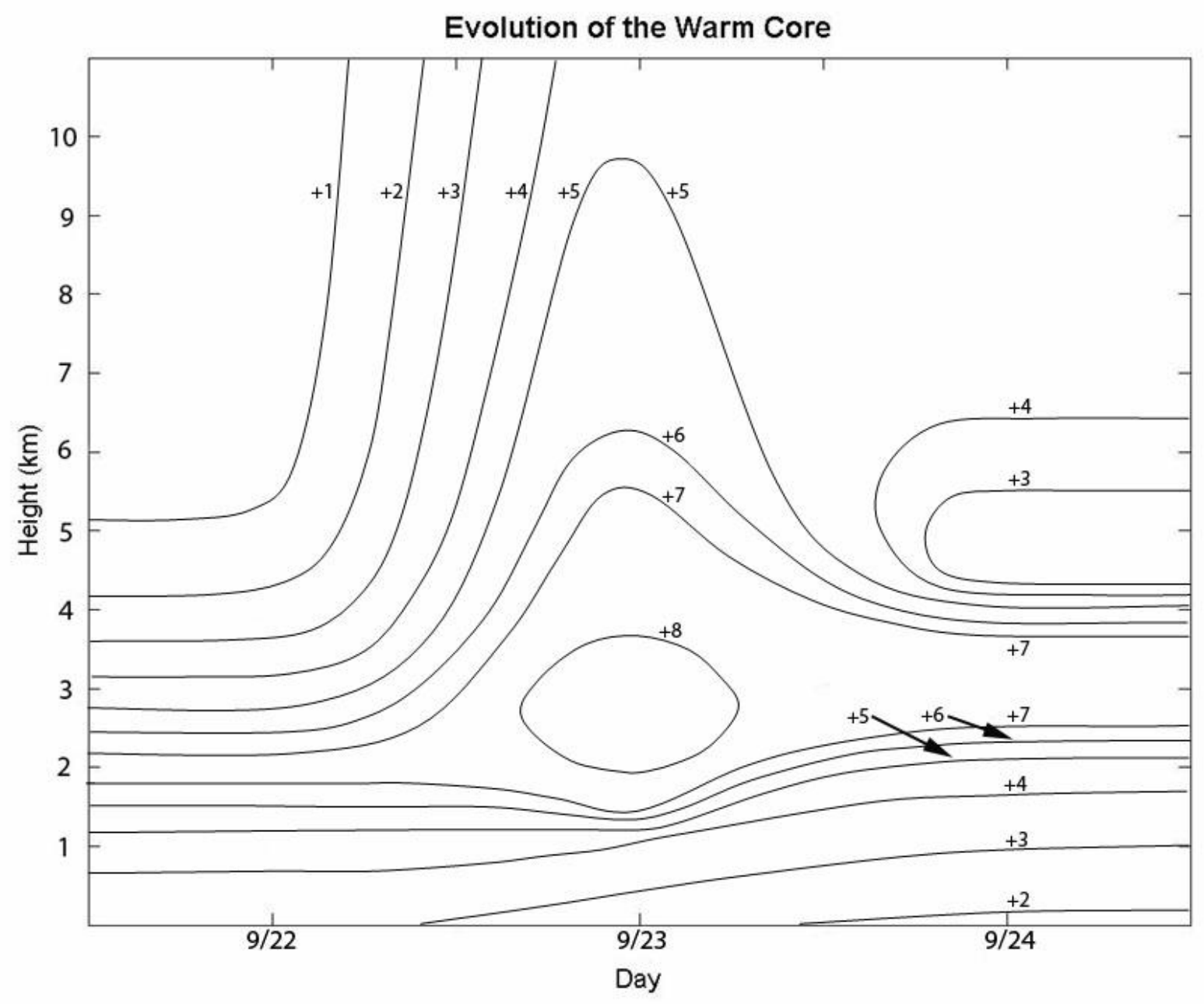

Figure 69. The evolution of the warm core as a function of height and day from the $22^{\text {nd }}$ to the $24^{\text {th }}$. Contours are of the warm core anomaly every 1 degree Kelvin. 


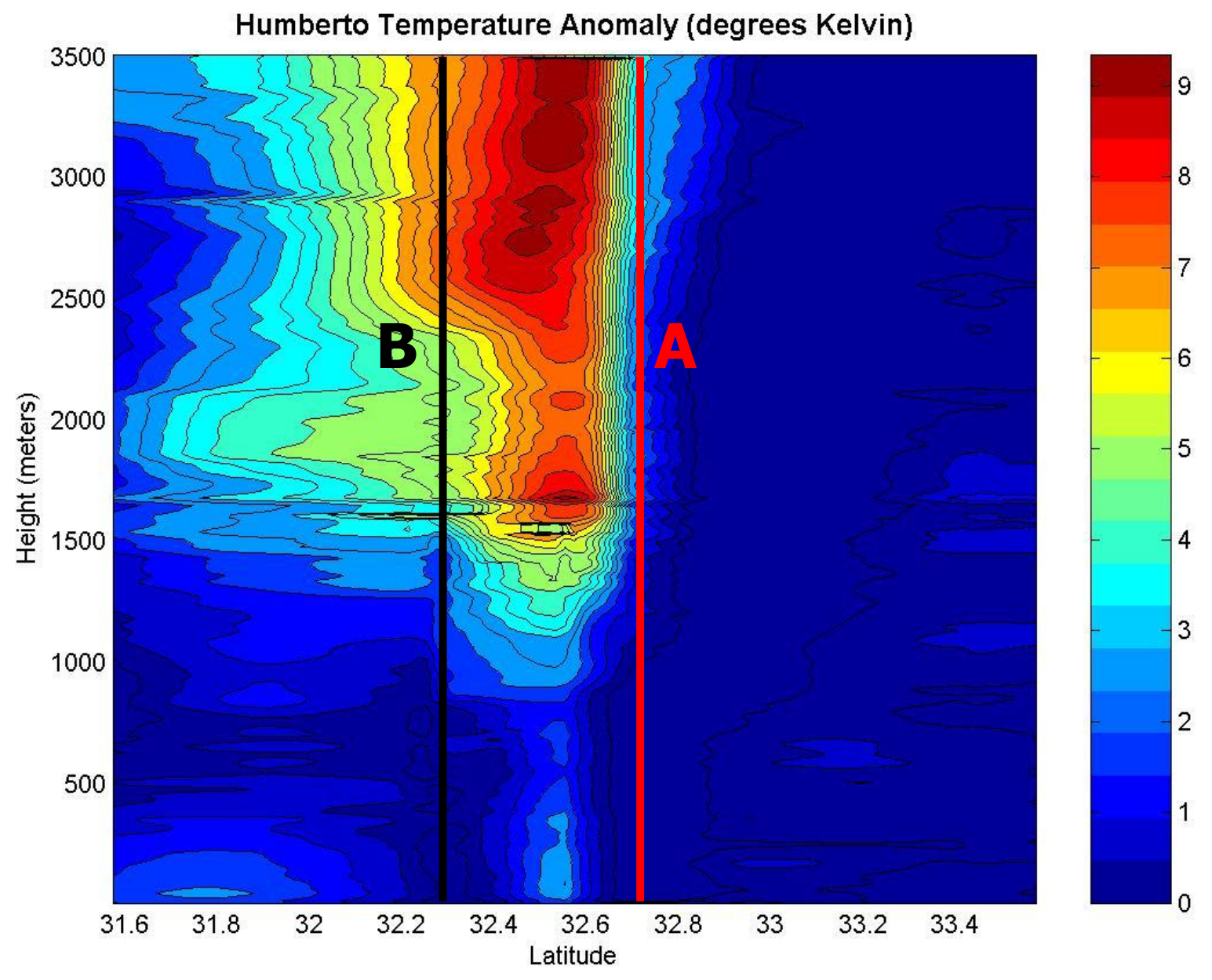

Figure 70. North to south vertical cross-section of the temperature perturbation (Kelvin) for Humberto on the $23^{\text {rd }}$. Color bar to right shows values in degrees Kelvin. Line "A" represents the latitudinal position of a GPS sonde jettisoned on the inner edge of the northern eyewall. Line "B" represents the latitudinal position of a GPS sonde jettisoned in the southern RMW. Circulation center is at $32.5^{\circ} \mathrm{N}$. 


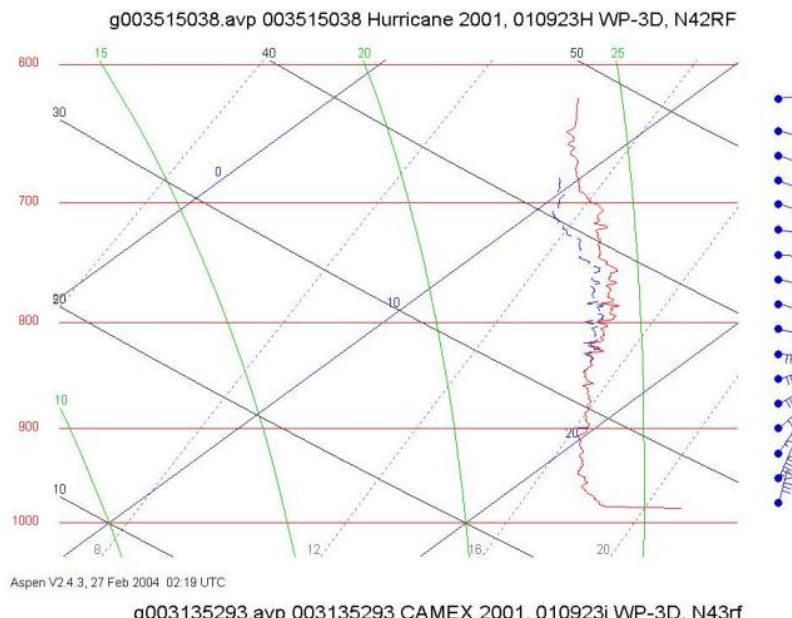

A

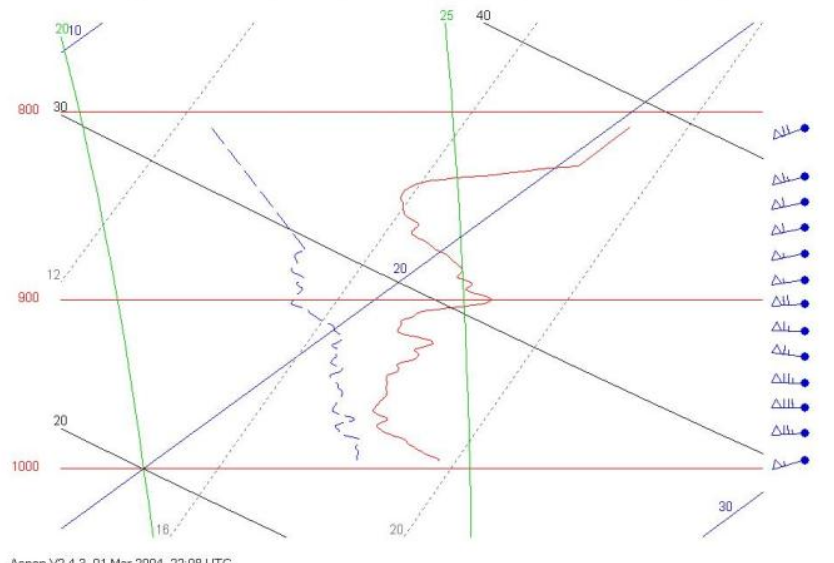

B

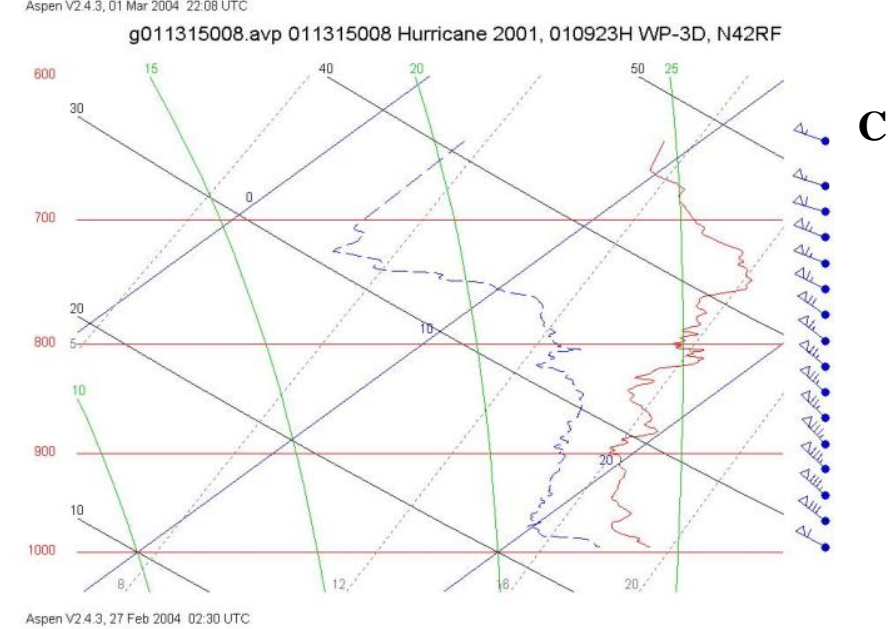

Figure 71. All soundings plotted on a skew-T log-P diagram. (A) Sounding taken at the position of line "A" from Fig. 70, jettisoned from the WP-3D. (B) Sounding taken at the position of line "B" from Fig. 70, jettisoned from the WP-3D. (C) Sounding taken just west of the sounding " $\mathrm{B}$ " in Fig. 70, jettisoned from the WP-3D. 


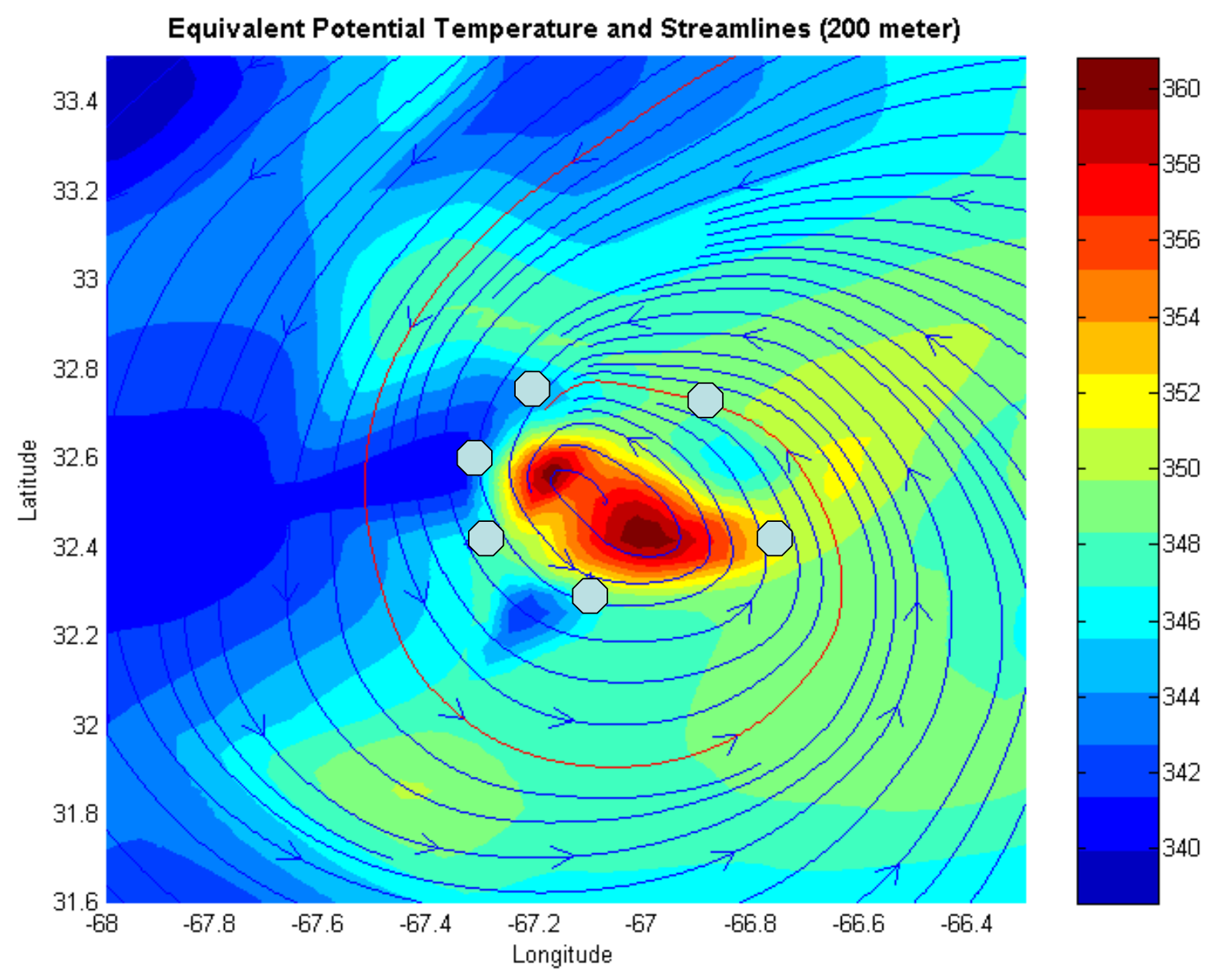

Figure 72. Close up plan view of $\theta_{\mathrm{e}}$ at $200 \mathrm{~m}$ and streamlines on 9/23/01. Color bar to right delineates magnitude (Kelvin). Grey dots show where the radius of maximum wind is located according to the low-level WP-3D. 

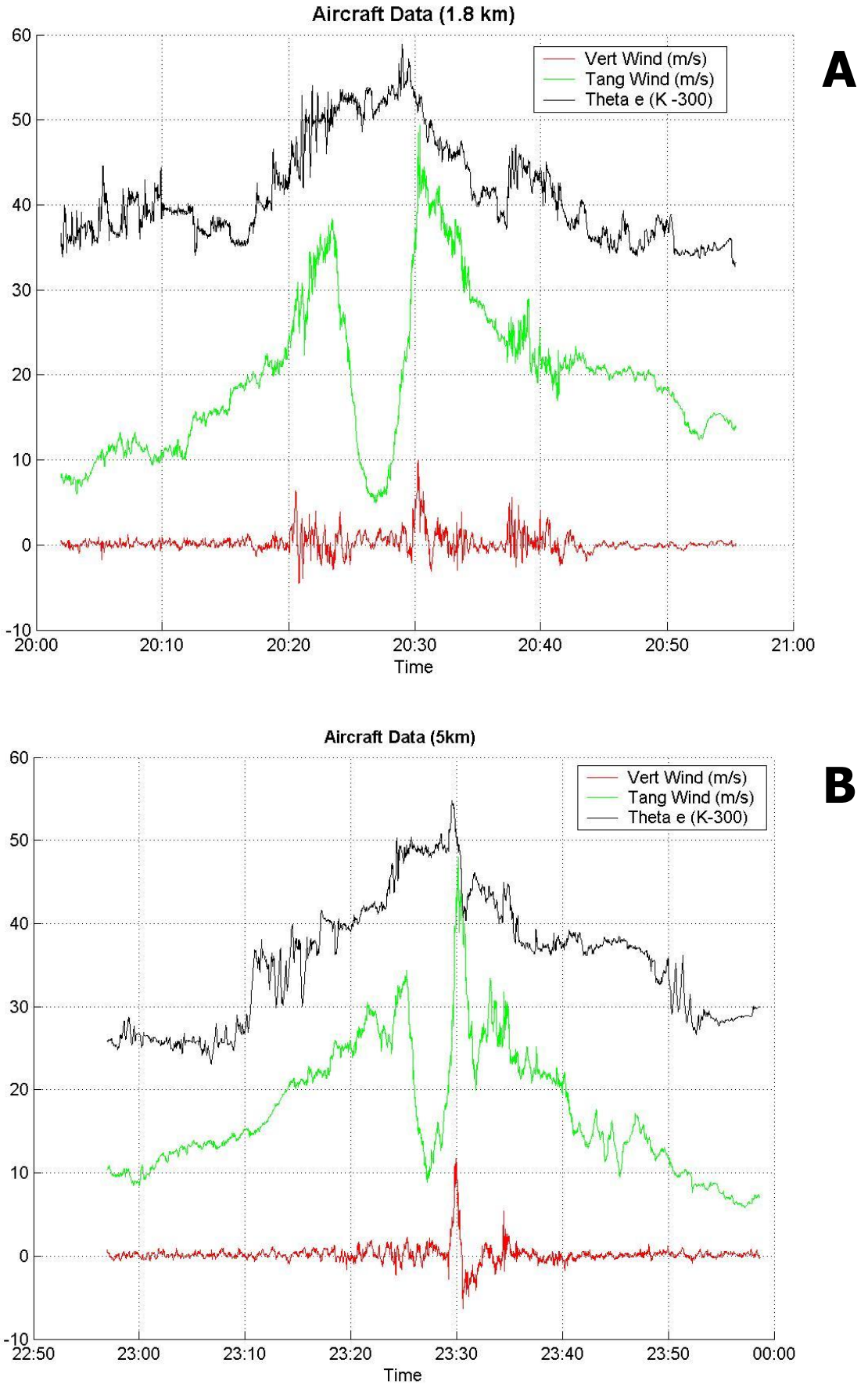

B

Figure 73. (A) South to north view of the vertical velocity, tangential winds, and equivalent potential temperature taken from the WP-3D flying at $1.8 \mathrm{~km}$ on $9 / 23 / 01$. (B) Southeast to northwest view taken from the WP-3D flying at $5 \mathrm{~km}$ on 9/23/01. 


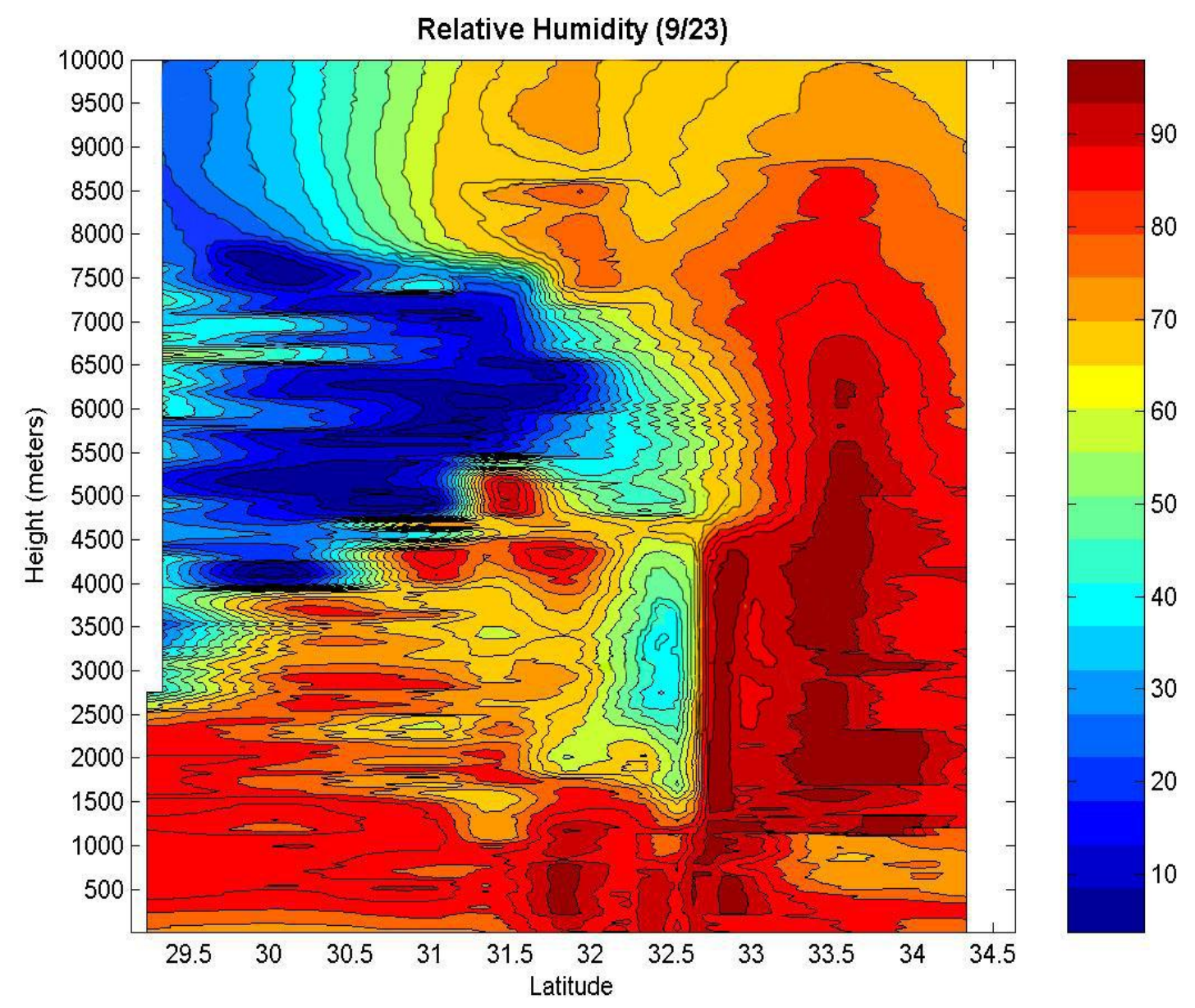

Figure 74. Vertical cross-section from north to south of the relative humidity on 9/23/01 from $0-10 \mathrm{~km}$. Color bar at right corresponds to the relative humidity. Eye is located at $32.5^{\circ} \mathrm{N}$. Southern eyewall located at $32.2^{\circ} \mathrm{N}$. Northern eyewall is located at $32.8^{\circ} \mathrm{N}$. 


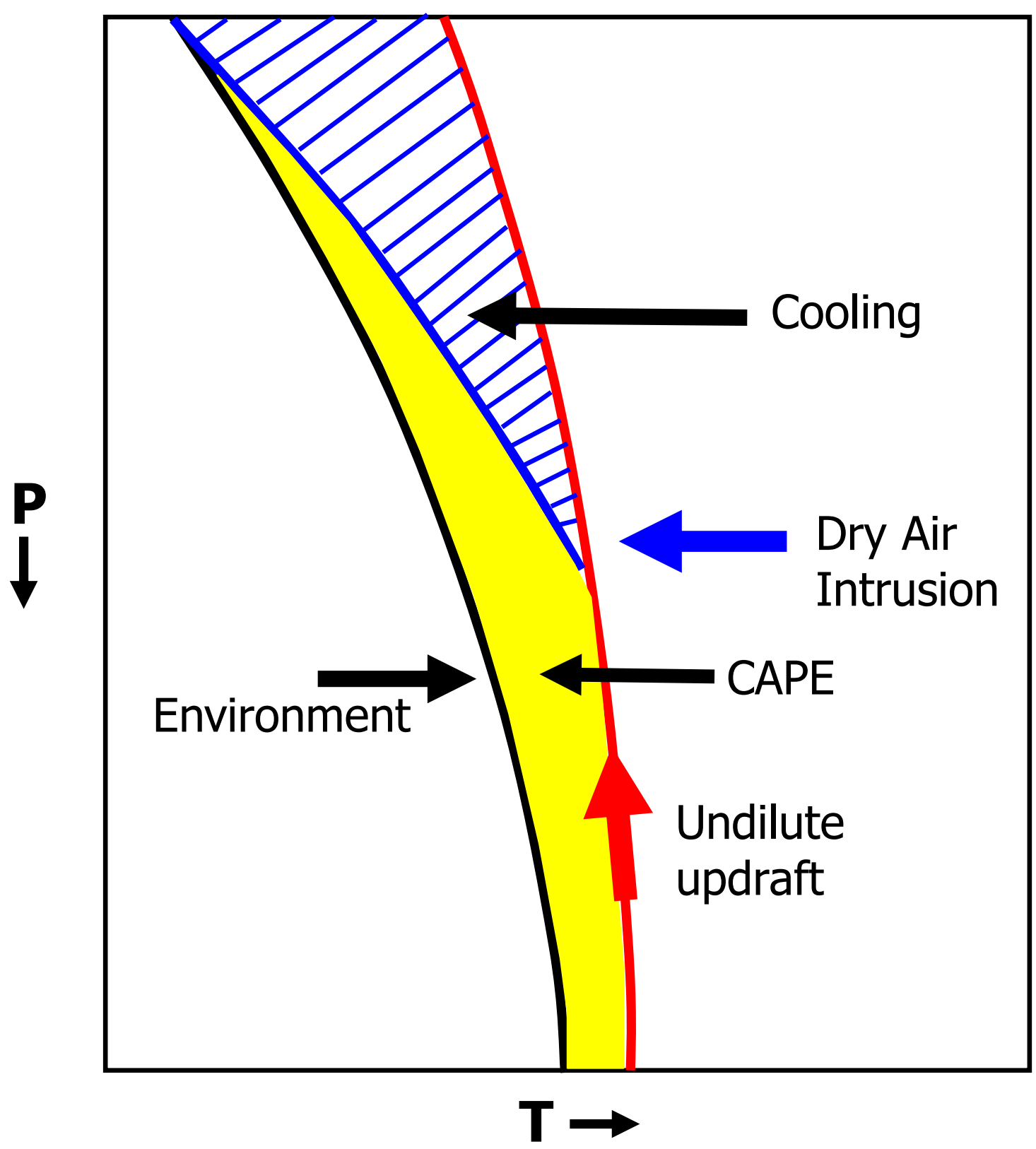

Figure 75. Schematic of the effect of dry air on an undiluted updraft containing CAPE. Pressure is on the y-axis and temperature is along the $\mathrm{x}$-axis. Red line is undiluted updraft. Black line is an environmental sounding representing a moist adiabat. Yellow area is CAPE. Blue hatched area is cooling due to dry air intrusion. 


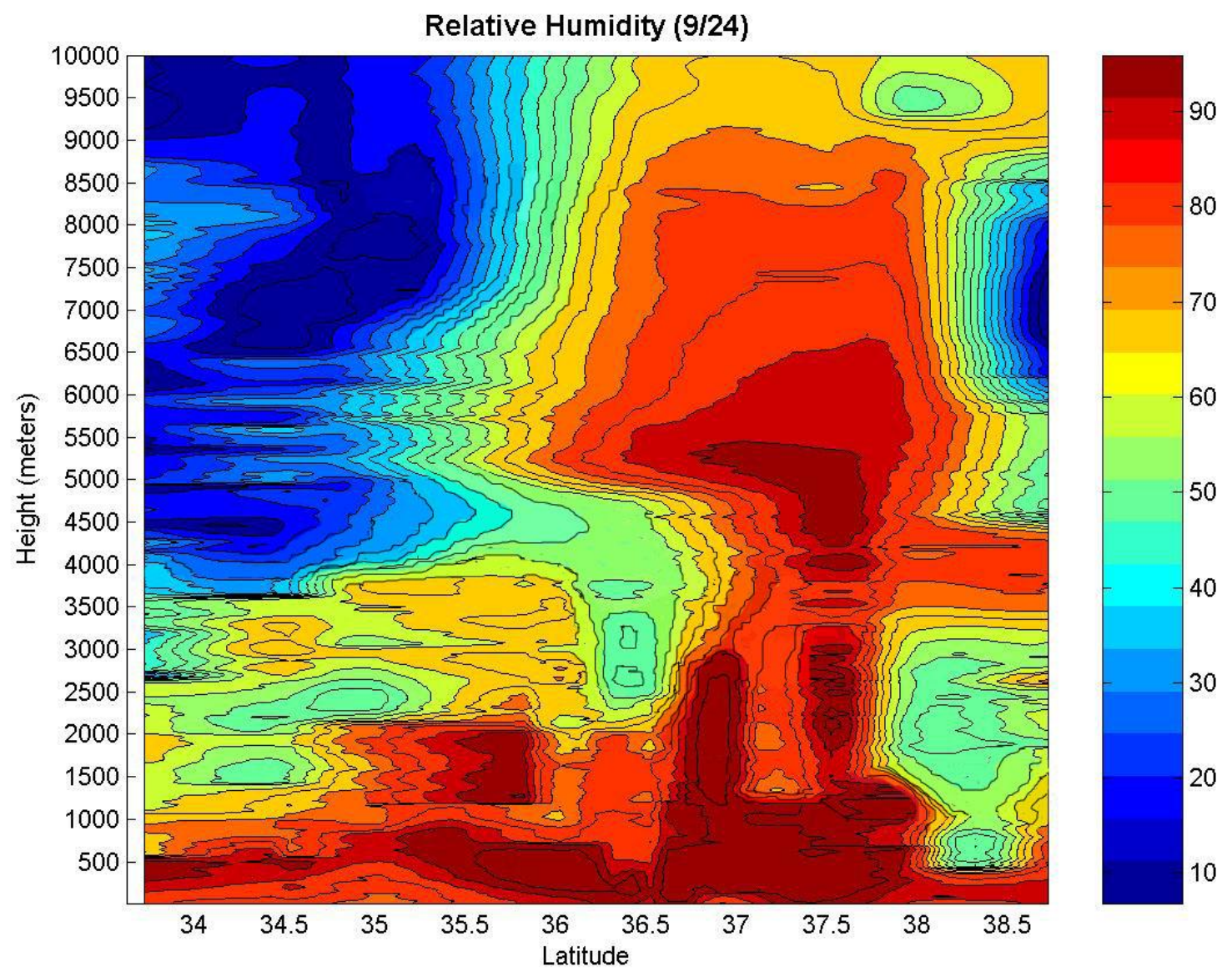

Figure 76. Vertical cross-section from north to south of the relative humidity on 9/24/01 from $0-10 \mathrm{~km}$. Color bar at right corresponds to the relative humidity. The eye is located at $36.4^{\circ} \mathrm{N}$. Southern eyewall is located at $36^{\circ} \mathrm{N}$. Northern eyewall is located at $36.8^{\circ} \mathrm{N}$. 


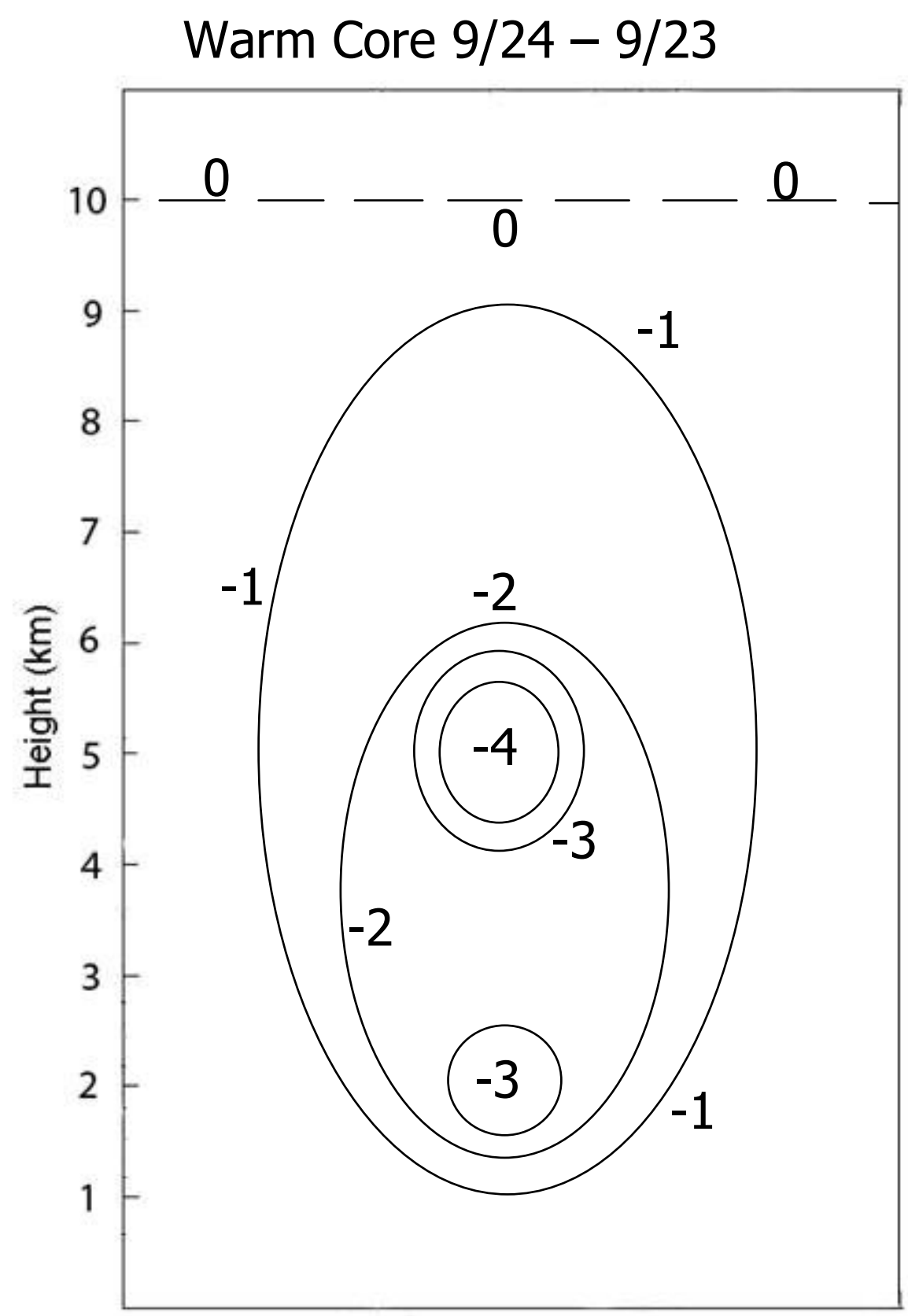

Figure 77. Schematic of the difference in the temperature perturbation between 9/24/01 and 9/23/01. Contours are every 1 degree Kelvin. 

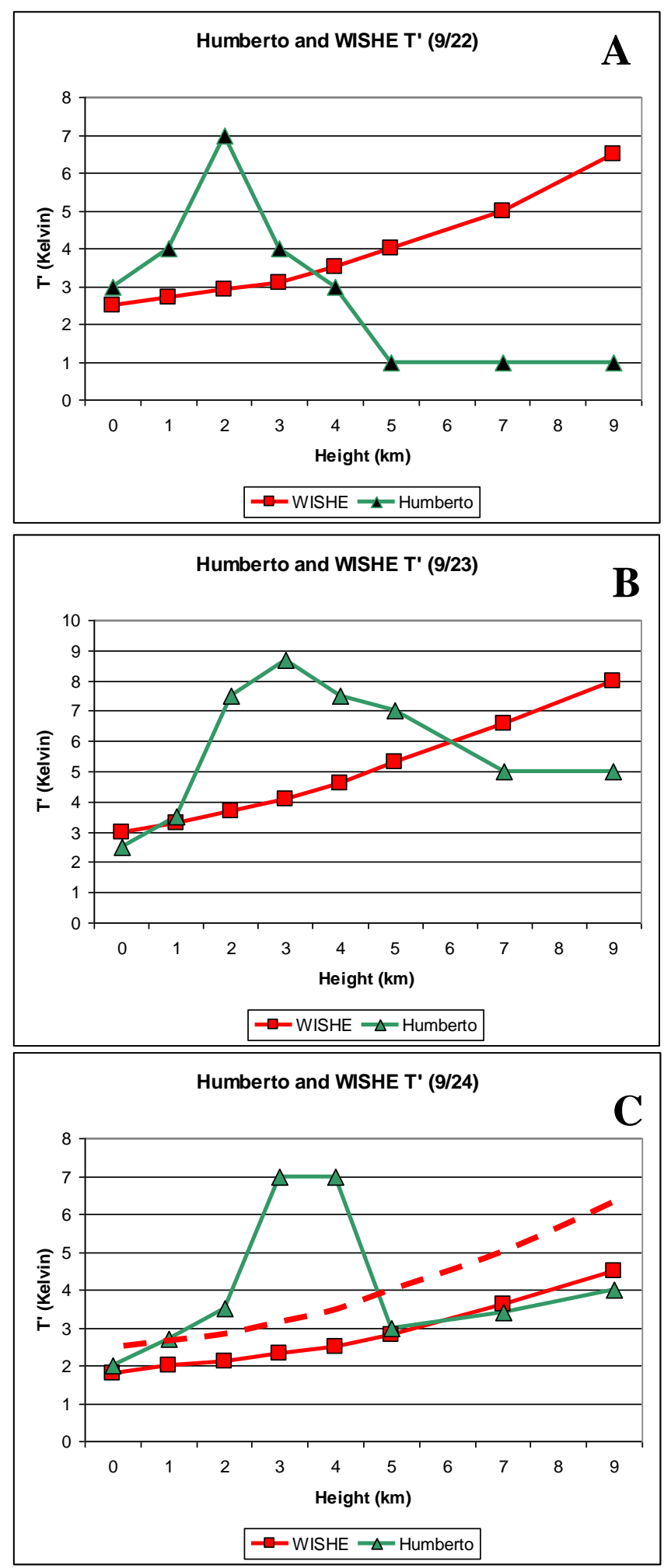

Figure 78. Comparison of Humberto temperature anomaly (green) with WISHE temperature anomaly (red). (A) 9/22/01 (B) 9/23/01 (C) 9/24/01. Red dashed line on $9 / 24 / 01$ is the WISHE temperature anomaly if the eye $\theta_{\mathrm{e}}$ is used instead of the eyewall. 


\section{Appendix}

\section{Divergence $200 \mathrm{hPa} 9 / 22 / 01$}

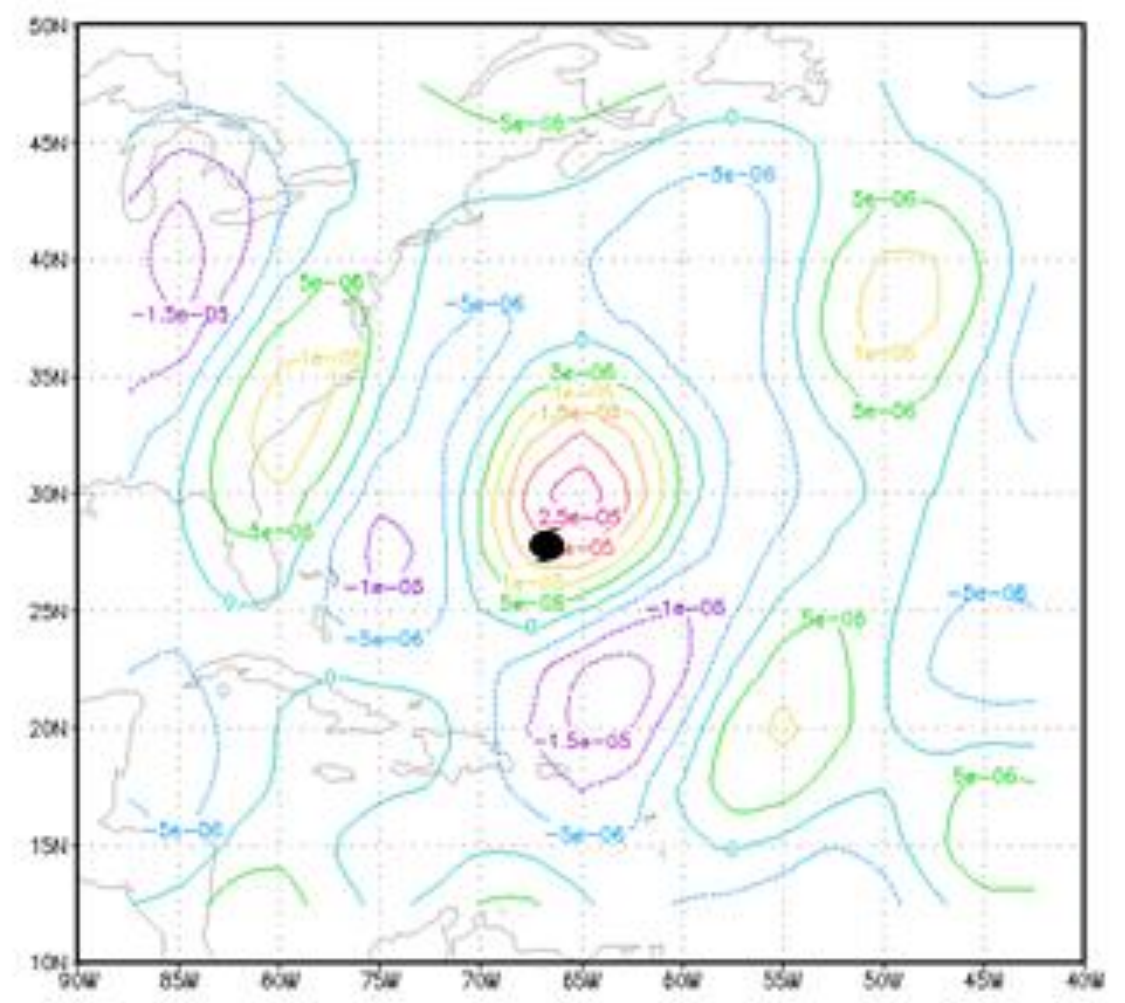

onos: ocuines

Figure 1. NCEP/NCAR Reanalysis displaying divergence $\left(\mathrm{s}^{-1}\right)$ at $200 \mathrm{hPa}$ geopotential at 00 UTC on September 22, 2001. Black dot shows low-level circulation center. 


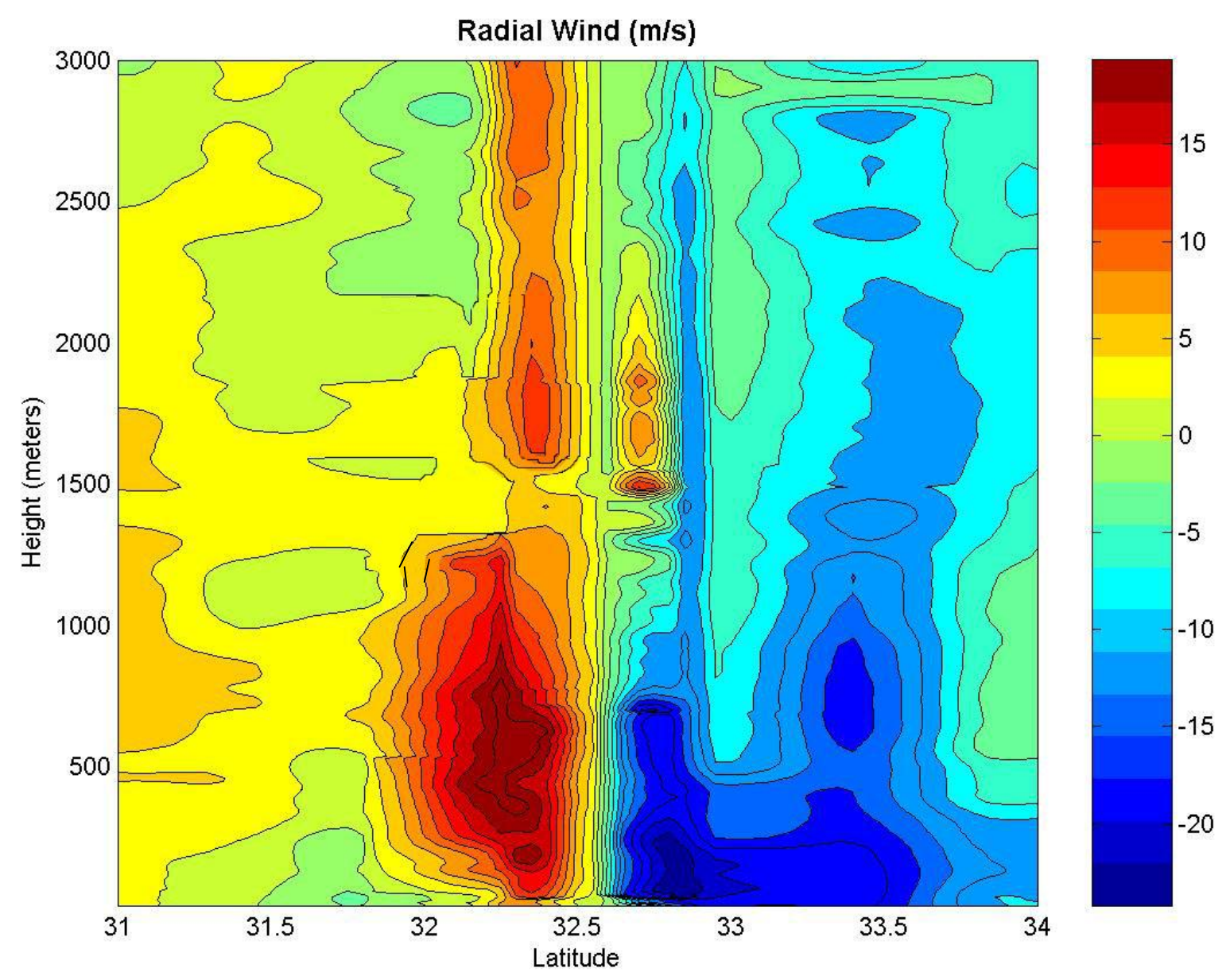

Figure 2. North to south vertical cross-section of the radial winds on 9/23/01. Color bar on right denotes the radial wind velocity $\left(\mathrm{m} \mathrm{s}^{-1}\right)$. 

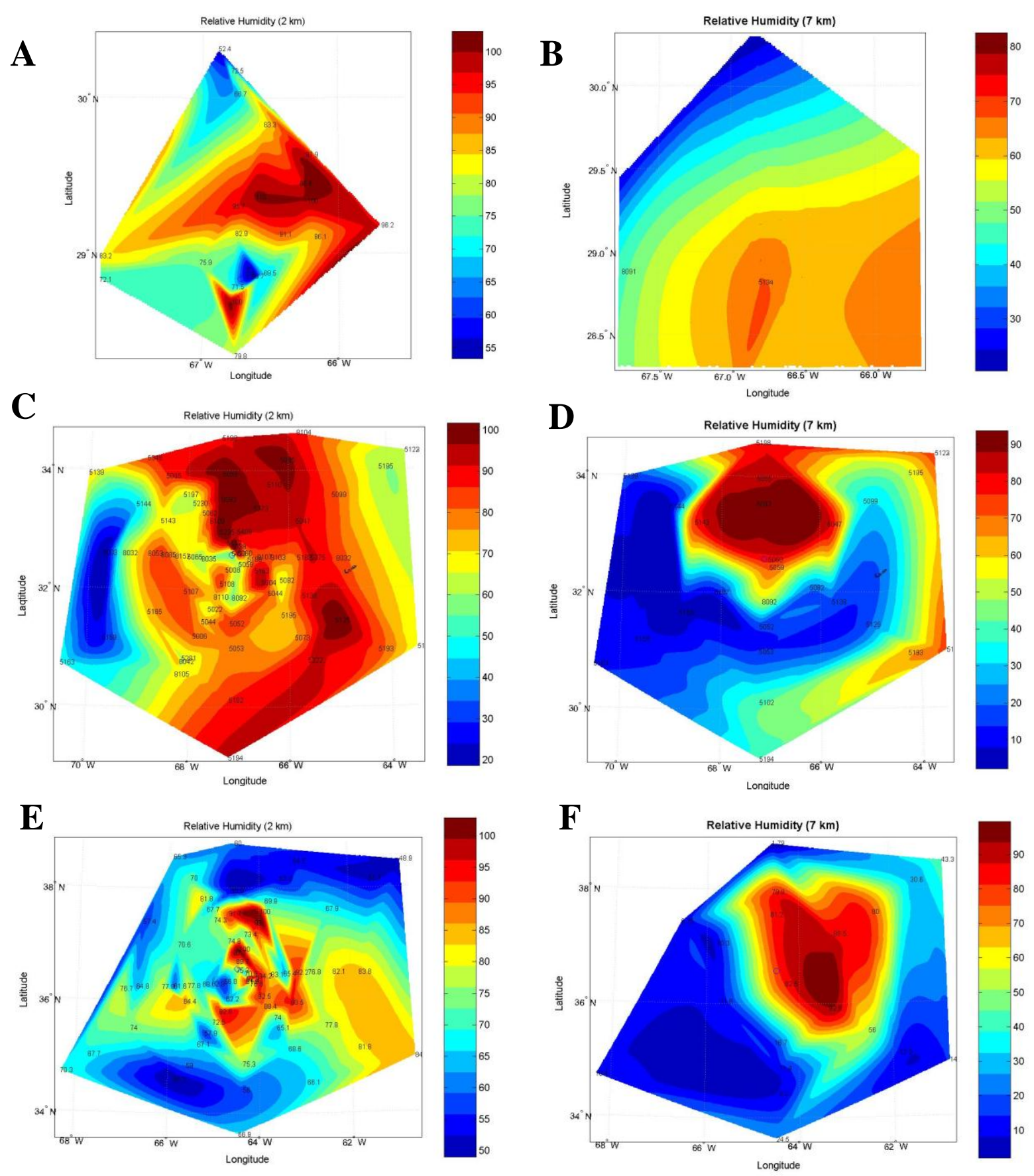

Figure 3. Plan views of relative humidity at 2 and $7 \mathrm{~km}$ for various days of the experiment. Color bar to right delineates the relative humidity. Note difference in horizontal scales for each day. (A) $2 \mathrm{~km} \mathrm{9/22/01} \mathrm{(B)} 7 \mathrm{~km} \mathrm{9/22/01} \mathrm{(C)} 2 \mathrm{~km} \mathrm{9/23/01} \mathrm{(D)}$ 7 km 9/23/01 (E) 2 km 9/24/01 (F) 7 km 9/24/01. 


\section{References}

Aberson, S. D., M. L. Black, R. A. Black, R. W. Burpee, J. J. Cione, C. W. Landsea, and F. D. Marks, 2006: Thirty years of tropical cyclone research with the NOAA P-3 aircraft. Bull. Amer. Meteor. Soc., 1039-1055.

Albrecht, B., and S. K. Cox, 1975: The large-scale response of the tropical atmosphere to cloud-modulated infrared heating. J. Atmos. Sci., 32, 16-24.

American Meteorological Society Statement on Hurricane Research and Forecasting, 2000: Bull. Amer. Meteor. Soc., 81, 1341- 1346.

Barnes, G. M., 2008: Atypical thermodynamic profiles in hurricanes. Mon. Wea. Rev., 136, 631-643. , and P. Fuentes, 2010: Eye excess energy and the rapid intensification of Hurricane Lili (2002). Mon. Wea. Rev., 138, 1446-1458.

, E. J. Zipser, D. Jorgensen, and F. D. Marks, 1983: Mesoscale and convective structure of a hurricane rainband. J. Atmos. Sci., 40, 2125-2137.

Barnes, S. L., 1964: A technique for maximizing details in numerical weather map analysis. J. Appl. Meteor., 3, 396-409.

Bates, J. R., 1973: A generalization of the CISK theory. J. Atmos. Sci., 30, 1509-1519.

Bender, M. A. 1997: The effect of relative flow on the asymmetric structure in the interior of hurricanes. J. Atmos. Sci., 54, 703-724.

Betts, A. K. and J. Simpson, 1987: Thermodynamic budget diagrams for the hurricane subcloud layer. J. Atmos. Sci., 44, 842-849.

Bister, M., and K. A. Emanuel, 1997: The genesis of Hurricane Guillermo: TEXMEX analyses and modeling study. Mon Wea Rev., 125, 2662-2682.

, M., and K. A. Emanuel, 1998: Dissipative heating and hurricane intensity. Meteor. Atmos. Phys., 65, 233-240.

Black, M. L., R. W. Burpee, and F. D. Marks, 1996: Vertical motion characteristics of tropical cyclones determined with airborne Doppler radial velocities. J. Atmos. Sci., 53, 1887-1909. 
, J. F. Gamache, F. D. Marks Jr., C. E. Samsury, and H. E. Willoughby, 2002: Eastern Pacific Hurricanes Jimena of 1991 and Olivia of 1994: The effect of vertical wind shear on structure and intensity. Mon. Wea. Rev., 130, 2291-2312.

Black, P. G., F. D. Marks Jr., and R. A. Black, 1986: Supercell structure in a tropical cyclone. Preprints, $23^{\text {rd }}$ Conf. on Radar Meteorology/Conf. on Cloud Physics, Snowmass, CO, Amer. Meteor. Soc., JP255-JP259.

Bogner, P. B., and G. M. Barnes, 2000: Conditional instability and shear for six hurricanes over the Atlantic Ocean. Wea. Forecasting, 15, 192-207.

Bolton, D., 1980: The computation of equivalent potential temperature. Mon. Wea. Rev., 108, 1046-1053.

Bosart, L. F., and F. S. Sanders, 1981: The Johnstown flood of July 1977: A long-lived convective system. J. Atmos. Sci., 38, 1616-1642.

, C. S. Velden, W. E. Bracken, J. Molinari, and P. G. Black, 2000: Environmental influences on the rapid intensification of hurricane Opal (1995) over the Gulf of Mexico. Mon. Wea. Rev., 128, 322-352.

Brandes, E. A., 1990: Evolution and structure of the 6-7 May 1985 mesoscale convective system and associated vortex. Mon. Wea. Rev., 118, 109-127.

Bryan, G. H., and J. M. Fritsch, 2000: Moist absolute instability: The sixth static stability state. Bull. Amer. Meteor., 81, 1207-1230.

Charney, J., 1973: Moveable CISK. J. Atmos. Sci., 30, 50-52.

Chen, S. C. and W. M. Frank, 1993: A numerical study of the genesis of extratropical convective mesovortices. Part I: Evolution and dynamics. J. Atmos. Sci., 50, 2401-2426.

Corbosiero, K. L. and J. Molinari, 2003: The relationship between storm motion, vertical wind shear, and convective asymmetries in tropical cyclones. J. Atmos. Sci., 60, 366-376.

Davidson, N. E., G. J. Holland, J. L. McBride, and T. D. Keenan, 1990: On the formation of AMEX tropical cyclones Irma and Jason. Mon. Wea. Rev., 118, 1981-2000.

DeMaria, M., 1996: The effect of vertical shear on tropical cyclone intensity change. $J$. Atmos. Sci., 53, 2076-2087.

, M. and J. Kaplan, 1994: A statistical hurricane intensity prediction scheme (SHIPS) for the Atlantic Basin. Wea. Forecasting, 9, 209-220. 
, and , 1999: An updated statistical hurricane intensity prediction scheme (SHIPS) for the Atlantic and Eastern North Pacific Basins. Wea. Forecasting, 14, 326-337.

, M. Mainelli, L. K. Shay, J. A. Knaff, and J. Kaplan, 2005: Further improvements to the statistical hurricane intensity prediction scheme (SHIPS). Wea. Forecasting, 20, 531-543.

Doswell, C. A., and E. N. Rasmussen, 1994: The effect of neglecting the virtual temperature correction on CAPE calculations. Wea. Forecasting, 9, 625-629.

Drury, S., and J. L. Evans, 1998: Modeling of tropical cyclone intensification as a result of interaction with middle-latitude troughs. Preprints, Symp. On Tropical Cyclone Intensity Change, Phoenix, AZ, Amer. Meteor. Soc., 65-72.

Eastin, D. E., W. M. Gray, and P. G. Black, 2005: Buoyancy of convective vertical motions in the inner core of intense hurricanes. Part II: Case studies. Mon. Wea. Rev., 133, 209-227.

Emanuel, K. A., 1986: An air-sea interaction theory for tropical cyclones. Part I: Steadystate maintenance. J. Atmos. Sci., 43, 585-604.

, 1989: The finite-amplitude nature of tropical cyclogenesis. J. Atmos. Sci., 46, 3431-3456.

Enagonio, J., and M. T. Montgomery, 2001: Tropical cyclogenesis via convectively forced vortex Rossby waves in a shallow water primitive equation model. $J$. Atmos. Sci., 58, 685-705.

Fairall, C. W., E. F. Bradley, J. E. Hare, A. A. Grachev, and J. B. Edson, 2003: Bulk parameterization of air-sea fluxes: Updates and verification for the COARE algorithm. J. Climate., 16, 571-591.

Fletcher, R. D., J. R. Smith, and R. C. Bundgaard, 1961: Superior photographic reconnaissance of tropical cyclones. Weatherwise, 14 (3), 103-109.

Fraedrich, K., and J. L. McBride, 1989: The physical mechanism of CISK and the freeride balance. J. Atmos. Sci., 46, 2642-2648.

Frank, W. M., 1977: The structure and energetics of the tropical cyclone. I. Storm structure. Mon. Wea. Rev., 105, 1136-1150. , 1998: Mechanisms of environmentally induced hurricane structure change. Preprints, Symp. On Tropical Cyclone Intensity Change, Phoenix, AZ, Amer. Meteor. Soc., 15-20. 
, and S. Chen, 1991: Simulations of vortex formation in convective weather systems. Preprints, $19^{\text {th }}$ Conf. Hurr. Trop. Meteor., Miami, Fl, Amer. Meteor. Soc., Boston, MA 02108,241-244.

, and E. A. Ritchie, 1999: Effects of environmental flow upon tropical cyclone structure. Mon. Wea. Rev., 127, 2044-2061.

, and 2001: Effects of vertical wind shear on the intensity and structure of numerically simulated hurricanes. Mon. Wea. Rev., 129, 2249-2269.

Franklin, J. L., S. J. Lord, and F. D. Marks, 1988: Dropwindsonde and radar observations of the eye of hurricane Gloria (1985). Mon. Wea. Rev., 116, 1237-1244.

Fritsch, F. N., and R. E. Carlson, 1980: Monotone piecewise cubic interpolation. SIAM J. Numer. Anal., 17, 238-246.

Fritsch, J. M., J. D. Murphy, and J. S. Kain, 1994: Warm core vortex amplification over land. J. Atmos. Sci., 51, 1780-1807.

Gamache, J. F., and R. A. Houze, Jr., 1982: Mesoscale air motions associated with a tropical squall-line. Mon. Wea. Rev., 110, 118-135.

Gray, W. M., 1968: Global view of the origin of tropical disturbances and storms. Mon. Wea. Rev., 96, 669-700. , 1975: Tropical cyclone genesis. Dept. of Atmos. Sci. Paper No. 234, Colo. State Univ., Fort Collins, CO, 121 pp. , 1979: 'Hurricanes: Their formation, structure and likely role in the tropical circulation', pp. 155-218 in Meteorology over the tropical oceans. Quart. J. Roy. Meteor. Soc. 155-218.

Hanley, D., J. Molinari, and D. Keyser, 2001: A composite study of the interactions between tropical cyclones and upper-tropospheric troughs. Mon. Wea. Rev., 129, 2570-2584.

Harr, P. A., M. S. Kalafsky, and R. L. Elsberry, 1996: Environmental conditions prior to formation of a midget tropical cyclone during TCM-93. Mon. Wea. Rev., 124, 1693-1710.

Hawkins, H. F., and D. T. Rubsam, 1968: Hurricane Hilda, 1964, II. Structure and budgets of the hurricane on October 1, 1964. Mon. Wea. Rev.., 96, 617-636. , and S. M. Imbembo, 1976: The structure of a small, intense hurricane: Inez, 1966. Mon. Wea. Rev.., 104, 418-442. 
Hendricks, E. A., and M. T. Montgomery, 2004: The role of "vortical" hot towers in the formation of tropical cyclone Diana (1984). J. Atmos. Sci., 61, 1209-1232.

Heymsfield, G. M., E. Ritchie, J. Simpson, J. Molinari, and L. Tian, 2006: Structure of highly sheared tropical storm Chantal during CAMEX-4. J. Atmos. Sci., 63, 268287.

Hock, T. F., and J. L. Franklin, 1999: The NCAR GPS dropwindsonde. Bull. Amer. Meteor. Soc., 80, 407-420.

Holland, G. J., and R. T. Merril, 1984: On the dynamics of tropical cyclone structural changes. Quart. J. Roy. Meteor. Soc., 110, 723-745.

, and R. Lukas, 2006: Program for improvements to hurricane intensity forecasts and impacts projections (HiFi). Report available from authors via web.

Holliday, C. R., A. H. Thompson, 1979: Climatological characteristics of rapidly intensifying typhoons. Mon. Wea. Rev., 107, 1022-1034.

Holton, J. R., 2004: An Introduction to Dynamic Meteorology . Elsevier Academic Press, pp. 47.

Houze, Jr., R. A., 1977: Structure and dynamics of a tropical squall-line system. Mon Wea. Rev., 105, 1540-1567.

Hubert, L. F., 1955: A case study of hurricane formation. J. Meteor., 12, 486-492.

Jones, S. C., 1995: The evolution of vortices in vertical shear: I: Initially barotropic vortices. Quart. J. Roy. Meteor. Soc., 121, 821-851.

Jordan, C. L., 1958: Mean soundings for the West Indies area, J. Meteor., 15, 91-97. ,1961: Marked changes in the characteristics of the eye of intense typhoons between the deepening and filling stages. J. Meteor., 18, 779-789.

Jorgensen, D. P., 1984a: Mesoscale and convective-scale characteristics of mature hurricanes. Part I: General observations by research aircraft. J. Atmos. Sci., 41, 1268-1285.

1984b: Mesoscale and convective-scale characteristics of mature hurricanes. Part II: Inner core structure of Hurricane Allen (1980). J. Atmos. Sci., 41, 1287-1311.

Kleinschmidt, E., Jr., 1951: Gundlagen einer Theorie des tropischen Zyklonen. Archiv fur Meteorologie, Geophysik und Bioklimatologie, Serie A, 4, 53-72. 
Koss, W. J., 1976: Linear stability analysis of CISK-induced disturbances: Fourier component eigenvalue analysis. J. Atmos. Sci., 33, 1195-1222.

Kossin, J. P., and M. D. Eastin, 2001: Two distinct regimes in the kinematic and thermodynamic structure of the hurricane eye and eyewall. J. Atmos. Sci., 58, 1079-1090.

Laing, A. G., and J. M. Fritsch, 1993a: Mesoscale convective complexes over the Indian monsoon region. J. Climate., 6, 911-919. , and 1993b: Mesoscale convective complexes in Africa. Mon. Wea. Rev., 121, 2254-2263.

Large, W. G., and S. Pond, 1982: Sensible and latent heat fluxes over the ocean. J. Phys. Oceanogr., 12, 464-482.

Leary, C. A., 1980: Temperature and humidity profiles in mesoscale unsaturated downdrafts. J. Atmos. Sci., 37, 1005-1012. , and R. O. R. Y. Thompson, 1976: A warm-core disturbance in the western Atlantic during BOMEX. Mon. Wea. Rev., 104, 443-452. , and E. N. Rappaport, 1987: The life cycle and internal structure of a mesoscale convective complex. Mon. Wea. Rev., 115, 1503-1527.

Lewis, B. M., and D. P. Jorgensen, 1978: Study of the dissipation of Hurricane Gertrude (1974). Mon. Wea. Rev., 106, 1288-1305.

Mak, M.-K., 1981: An inquiry on the nature of CISK, Part I. Tellus, 33, 531-537.

Marks, F. D. Jr., 1985: Evolution of the structure of precipitation in Hurricane Allen. Mon. Wea. Rev., 113, 909-930.

, R. A. Houze, and J. F. Gamache, 1992: Dual-aircraft investigation of the inner core of Hurricane Norbert. Part I: Kinematic structure. J. Atmos. Sci., 49, 919942.

, L. K. Shay, and PDT-5, 1998: Landfalling tropical cyclones: Forecast problems and associated research opportunities. Bull. Amer. Meteor. Soc., 79, 305-323.

Malkus, J. S., and H. Riehl, 1960: On the dynamics and energy transformation in steadystate hurricanes. Tellus, 12, 1-20.

McBride, J. L. and R. Zehr, 1981: Observational analysis of tropical cyclone formation. Part II: Comparison of non-developing versus developing systems. J. Atmos. Sci., 38, 1132-1151. 
Menard, R. D. and J. M. Fritsch, 1989: A mesoscale convective complex-generated inertially stable warm core vortex. Mon. Wea. Rev., 117, 1237-1261.

Miller, B. I., 1964: A study of the filling of hurricane Donna (1960) over land. Mon. Wea. Rev., 9, 389-406.

Miller, D., and J. M. Fritsch, 1991: Mesoscale convective complexes in the western Pacific region. Mon. Wea. Rev., 119, 2978-2992.

Molinari, J. and D. Vollaro, 1989: External influences on hurricane intensity. Part I: Outflow layer eddy angular momentum fluxes. J. Atmos. Sci., 46, 1093-1105. , and _ 2010: Notes and correspondence: Distribution of helicity, CAPE, and shear in tropical cyclones. J. Atmos. Sci., 67, 274-284.

, S. Skubis, and D. Vollaro, 1995: External influences on hurricane intensity. Part III: Potential vorticity structure. J. Atmos. Sci., 52, 3593-3606.

, D. Vollaro, and K. L. Corbosiero, 2004: Tropical cyclone formation in a sheared environment: A case study. J. Atmos. Sci., 61, 2493-2509.

Moller, J. D. and M. Montgomery, 2000: Tropical cyclone evolution via potential vorticity anomalies in a three-dimensional balance model. J. Atmos. Sci., 57, 3366-3387.

Moncrieff, M. W., and J. S. A. Green, 1972: The propagation and transfer properties of steady convective overturning in shear. Quart. J. Roy. Meteor. Soc., 98, 336-353.

Montgomery, M. T., and C. Lu, 1997: Free waves on barotropic vortices. Part I: Eigenmode Structure. J. Atmos. Sci., 54, 1868-1885.

, and R. J. Kallenbach, 1997: A theory for vortex Rossby waves and its application to spiral bands and intensity changes in hurricanes. Quart. J. Roy. Meteor. Soc., 123, 435-465. , and J. Enagonio, 1998: Tropical cyclogenesis via convectively forced vortex Rossby waves in a three-dimensional quasi-geostrophic model. J. Atmos. Sci., 55, 3176-3207. , and J. L. Franklin, 1998: An assessment of the balance approximation in hurricanes. J. Atmos. Sci., 55, 2193-2200. 
, M. M. Bell, S. D. Aberson, and M. L. Black 2006: Hurricane Isabel (2003): New insights into the physics of intense storms. Part I. Bull. Amer. Meteor. Soc., 87, 1335-1347.

, M.E. Nicholls, T. A. Cram, and A. B. Saunders, 2006: A vortical hot tower route to tropical cyclogenesis, J. Atmos. Sci., 63, 355-386.

National Advisory Board, 2007: Hurricane Warning: The critical need for a national hurricane research initiative. [available from NSF.]

Persing, J., M. T. Montgomery, and R. E. Tuleya, 2002: Environmental interactions in the GFDL hurricane model for Hurricane Opal. Mon. Wea. Rev., 130, 298-317.

Powell, M. D., 1990: Boundary layer structure and dynamics in outer hurricane rainbands. Part II: Downdraft modification and mixed layer recovery. Mon. Wea. Rev., 118, 918-938.

Reasor, P. D., and M. T. Montgomery, 2005: Mesoscale observations of the genesis of Hurricane Dolly (1996). J. Atmos. Sci., 62, 3151-3171.

Riehl, H., 1948: On the formation of typhoons. J. Meteor., 5, 247-264. , 1950: A model for hurricane formation. J. Appl. Phys., 21, 917-925. , and J. S. Malkus, 1961: Some aspects of Hurricane Daisy, 1958. Tellus, 13, 181213.

Ritchie, E. A., and G. J. Holland, 1997: Scale interactions during the formation of Typhoon Irving. Mon. Wea. Rev., 128, 2560-2574.

, and R. L. Elsberry, 2001: Simulations of the transformation stage of the extratropical transition of tropical cyclones. Mon. Wea. Rev., 129, 1462-1480.

Rodgers, F. R., and J. M. Fritsch, 2001: Surface cyclogenesis from convectively driven amplification of midlevel mesoscale convective vortices. Mon. Wea. Rev., 129, 605-637.

Sadler, J. C., 1978: Mid-season typhoon development and intensity changes and the tropical upper tropospheric trough. Mon. Wea. Rev., 106, 1138-1137.

Schubert, W. H., M. T. Montgomery, R. K. Taft, T. A. Guinn, S. R. Fulton, J. P. Kossin, and J. P. Edwards, 1999: Polygonal eyewalls, asymmetric eye contraction, and potential vorticity mixing in hurricanes. J. Atmos. Sci., 56, 1198-1223. 
, C. M. Rozoff, J. L. Vigh, B. D. McNoldy, and J. P. Kossin., 2007: On the distribution of subsidence in the hurricane eye. Quart. J. Roy. Meteor. Soc., 133, 595-605.

Shapiro, L. J., 1983: The asymmetric boundary layer flow under a translating hurricane. J. Atmos. Sci., 40, 1984-1998. , and H. E. Willoughby, 1982: The response of balanced hurricanes to local sources of heat and momentum. J. Atmos. Sci., 39, 378-394.

Simpson, J., E. Ritchie, G. J. Holland, J. Halverson, and S. Stewart, 1997: Mesoscale interactions in tropical cyclone genesis. Mon. Wea. Rev., 125, 2643-2661.

Stossmeister, G. J. and G. M. Barnes, 1992: The development of a second circulation center within tropical storm Isabel (1985). Mon. Wea. Rev., 686, 685-697.

Stull, R. B., 2000: Meteorology for Scientists and Engineers. Brooks/Cole, pp. 109-110.

Syono, S., and M. Yamasaki, 1966: Stability of symmetrical motions driven by latent heat release by cumulus convection under the existence of surface friction. $J$. Meteor. Soc. Japan., 44, 353-375.

Velasco, I., and J. M. Fritsch, 1987: Mesoscale convective complexes in the Americas. J. Geophys. Res., 92, 9591-9613.

Ventham, J. D. and B. Wang, 2007: Large-scale flow patterns and their influence on the intensification rates of western north pacific tropical storms. Mon. Wea. Rev., 135, $1110-1127$.

Wang, J., 2005: Evaluation of the dropsonde humidity sensor using data from DYCOMSII and IHOP_2002. J. Atmos. Oceanic. Technol., 22, 247-257.

Weatherford, C. L. and W. M. Gray, 1988: Typhoon structure as revealed by aircraft reconnaissance. Part II: Structural variability. Mon. Wea. Rev., 116, 1032-1043.

Willoughby, H. E., 1998: tropical cyclone eye thermodynamics. Mon. Wea. Rev., 126, 3053-3067. , and M. B. Chelmow, 1982: Objective determination of hurricane tracks from aircraft observations. Mon. Wea. Rev., 110, 1298-1305.

, J. A. Clos., and M. G. Shoreibah, 1982: Concentric eye walls, secondary wind maxima, and the evolution of the hurricane vortex. J. Atmos. Sci., 39, 395-411.

Winn-Nielsen, A., 1993: Studies of CISK. Atmosfera, 6, 51-77. 
Wroe, R. and G. M. Barnes, 2003: Inflow layer energetics of Hurricane Bonnie (1998) near landfall. Mon. Wea. Rev., 131, 1600-1612.

Yanai, M. 1961: A detailed analysis of typhoon formation. J. Met. Soc. Japan., 39, 187213.

Zhang, D. L., and E. Altshuler, 1999: The effects of dissipative heating on hurricane intensity. Mon. Wea. Rev., 127, 3032-3038.

, Y. Liu, and M. K. Yau, 2002: A multiscale numerical study of Hurricane Andrew (1992). Part V: Inner-core thermodynamics. Mon. Wea. Rev., 130, 27452763.

Zipser, E. J., 1977: Mesoscale and convective-scale downdrafts as distinct components of squall-line structure. Mon. Wea. Rev., 105, 1568-1589.

Zipser, E. J., R. J. Meitin, and M. A. LeMone, 1981: Mesoscale motion fields associated with a slowly moving GATE convective band. J. Atmos. Sci., 38, 1725-1750. 